\title{
The Full Two-Loop R-parity Violating Renormalization Group Equations for All Minimal Supersymmetric Standard Model Couplings
}

\author{
A.F. Kord and A. Yazdanian \\ Department of Physics, Sabzevar Tarbiat Moallem University, P.O.Box 397, Sabzevar, Iran \\ farzaneh@sttu.ac.ir
}

\begin{abstract}
We present the full two-loop $\beta$-functions for the minimal supersymmetric standard model couplings, extended to include R-parity violating couplings through explicit R-parity violation.
\end{abstract}

\section{Introduction}

The supersymmetric extension [1, 2] of the Standard Model(SM) which introduces new scalar particles for SM fermions and new fermionic particles for SM bosons is a model which baryon $(B)$ and lepton $(L)$ number are not automatically conserved in the renormalisable Lagrangian. In the SM, the baryon and lepton number are conserved automatically because it is impossible to write down renormalizable gauge-invariant interactions that violate baryon and lepton number. However, in supersymmetric extensions of the SM new interactions are allowed to violate baryon or lepton number, so a discrete symmetry is often imposed to prohibit the unwanted interactions that violate $B$ and/or $L$ and lead to unacceptably large effects [3]. The most common discrete symmetry is $R$-parity which is defined 4 :

$$
R_{p}=(-1)^{2 S+3 B+L},
$$

where $S$ is the spin of the particle. All SM particles including the two scalar Higgs doublets are even under this transformation, and their superpartners are odd, which forces SUSY particles to always be made in pairs and forbids decay of the Lightest Supersymmetric Particle (LSP).

Alternatively, since R-conservation is not theoretically motivated by any known principle one can allow the $B$ and $L$ violating interactions to remain in the susy lagrangian, and constrain the couplings to be consistent with present experimental data. Both present of possible baryon and lepton number violating couplings may lead to unacceptably large effects such as proton decay. However; one can consider scenario which allow $B$ or $L$ violating interactions [4]. Therefore, the possibility of R-nonconservation deserves consideration. If we require a supersymmetric Standard Model which is only minimal in particle content the superpotential is modified to allow for additional Rparity violating interactions. R-parity violating interactions come from either superpotential terms which violate baryon number, separate terms which violate leptonnumber, or from soft supersymmetry breaking terms. There are different kinds of such terms, of dimensions 4,3 , or 2 , with a potentially rich flavour structure. 
The rich phenomenology implied by $R$-parity violation has now gained full attention in the search for supersymmetry. The R-parity violating terms in supersymmetry have theoretical and phenomenological implications in supersymmetric theories [6],such as:generating majorana neutrino masses, mediating various flavour and lepton number violating processes, modifying the signatures of supersymmetric particles at colliders, and possibility of decay of the lightest supersymmetric particle(LSP). It can also modify the Higgs sector [7, 8].

All parameters of the MSSM lagrangian such as gauge couplings and Yukawa couplings are scale dependent when one renormalizes the theory and replaces the bare parameters of the theory with running parameters. They are energy, or equivalently distance, dependent through virtual corrections. One can relate the values of these parameters at different scales using the Renormalisation Group Equations(RGEs), which involve the $\beta$-functions. We know low-energy supersymmetry is a perturbative theory, which relates observed phenomena at low energies to physics at very high-energy scales. For example, it is remarkable that in the MSSM, the three gauge couplings appear to unify at scale $2-3 \times 10^{16} \mathrm{GeV}$ (the GUT scale). With the parameters of the model specified at the input GUT scale by some candidate organizing principle, one can run the couplings and masses down to low energies using the renormalization group equations and make predictions about the sparticle masses and other low-energy phenomena. Therefore the knowledge of the $\beta$-functions and anomalous dimensions of the theory is vital. The gauge $\beta$ functions and the matter multiplet anomalous dimensions have been calculated up to four and three loop respectively [9, 10] in the R-parity conserving (RPC) case. The full three loop $\beta$-functions including soft $\beta$-functions have been presented in 11 . The two loop gauge $\beta$ functions and anomalous dimensions in the R-parity violating (RPV) case have been calculated in 13 . In particular, Ref 12 contained a complete set of one-loop $\beta$-functions for RPV parameters. The full two loop $\beta$-functions for the baryon number violating (BNV) couplings have been presented in [14. In this paper, we shall write down explicitly all possible R-parity violating terms in the framework of the Minimal Supersymmetric Standard Model, assuming the most general breaking of R-parity, and then will complete results of two loop $\beta$-functions for all $R$-parity violating parameters of the MSSM.

\section{Beta Functions}

For a general $\mathrm{N}=1$ supersymmetric gauge theory with superpotential

$$
W=\frac{1}{2} \mu^{i j} \Phi_{i} \Phi_{j}+\frac{1}{6} Y^{i j k} \Phi_{i} \Phi_{j} \Phi_{k}+L^{i} \Phi_{i}
$$

the standard soft supersymmetry-breaking scalar terms are as follows

$$
-\mathcal{L}_{\text {soft }}=\frac{1}{2} b^{i j} \phi_{i} \phi_{j}+\frac{1}{6} h^{i j k} \phi_{i} \phi_{j} \phi_{k}+\frac{1}{2}\left(m^{2}\right)_{i}^{j} \phi^{i} \phi_{j}+\frac{1}{2} M \bar{\lambda} \lambda+h . c .
$$

Here $\lambda$ is the gaugino and $M$ is its mass. The renormalization group equations for the gauge coupling and the superpotential parameters are given by:

$$
\beta_{g}=\mu \frac{d g}{d \mu}, \quad \beta_{i j k}=\mu \frac{d Y_{i j k}}{d \mu}, \quad \beta_{i j}=\mu \frac{d \mu_{i j}}{d \mu} \text { and } \beta_{i}=\mu \frac{d L_{i}}{d \mu}
$$


where $\mu$ is the renormalization mass scale. The $\beta$-functions $\beta_{i j k}, \beta_{i j}$ and $\beta_{i}$ are determined by the anomalous dimension $(\gamma)$ of the chiral supermultiplet $\Phi_{i}$ as follows:

$$
\begin{aligned}
& \beta^{i}=L^{j} \gamma_{j}^{i} \\
& \beta^{i j}=\mu^{i k} \gamma_{k}^{j}+\mu^{k j} \gamma_{k}^{i} \\
& \beta^{i j k}=Y^{i j l} \gamma_{l}^{k}+Y^{i l k} \gamma_{l}^{j}+Y^{l j k} \gamma_{l}^{i} .
\end{aligned}
$$

The one loop results for the gauge coupling $\beta_{g}$ and $\gamma$ are given by:

$$
16 \pi^{2} \beta_{g}^{(1)}=g Q, \quad 16 \pi^{2}\left(\gamma^{(1)}\right)_{j}^{i}=P_{j}^{i},
$$

where $Q$ and $P_{j}^{i}$ are given by:

$$
\begin{aligned}
& Q=T(R)-3 C(G) \\
& P_{j}^{i}=\frac{1}{2} Y^{i k l} Y_{j k l}-2 g^{2} C(R)_{j}^{i},
\end{aligned}
$$

where $Y_{i j k}=\left(Y^{i j k}\right)^{*}$ and $T(R)$ is the Dynkin index summed over all chiral multiplets. The Casimir invariants $C(G)$ and $C(R)_{j}^{i}$ are defined by:

$$
T(R) \delta_{a b}=\operatorname{Tr}\left(R_{a} R_{b}\right), \quad C(G) \delta_{a b}=f_{a c d} f_{b c d}, \quad \text { and } C(R)_{j}^{i}=\left(R_{a} R_{a}\right)_{j}^{i} .
$$

The two loop $\beta$-functions for the dimensionless couplings are given by:

$$
\begin{aligned}
& \left(16 \pi^{2}\right)^{2} \beta_{g}^{(2)}=2 g^{5} C(G) Q-2 g^{3} r^{-1} C(R)_{j}^{i} P_{i}^{j}, \\
& \left(16 \pi^{2}\right)^{2} \gamma_{j}^{(2) i}=\left[-Y_{j m n} Y^{m p i}-2 g^{2} C(R)_{j}^{p} \delta_{n}^{i}\right] P_{p}^{n}+2 g^{4} C(R)_{j}^{i} Q,
\end{aligned}
$$

where $r$ is the number of generators of the symmetry group, and $Q$ and $P_{j}^{i}$ are defined above.

The complete exact results for the soft -functions are given by [15, 16, 17]:

$$
\begin{aligned}
& \beta_{b}^{i j}=\gamma_{l}^{i} b^{l j}+\gamma_{l}^{j} b^{i l}-2 \gamma_{1}^{i} \mu^{l j}-2 \gamma_{1}^{j}{ }_{l} \mu^{i l} \\
& \beta_{h}^{i j k}=\gamma_{l}^{i} h^{l j k}+\gamma_{l}^{j} h^{i l k}+\gamma_{l}^{k} h^{i j l}-2\left(\gamma_{1{ }_{l}} Y^{l j k}+\gamma_{1}^{j}{ }_{l} Y^{i l k}+\gamma_{1}^{k}{ }_{l} Y^{i j l}\right) \\
& \beta_{M_{a}}=2 \Theta\left(\frac{\beta_{g_{a}}}{g_{a}}\right) \\
& \left(\beta_{m^{2}}\right)^{i}{ }_{j}=\left[\triangle+X \frac{\partial}{\partial g_{a}}\right] \gamma^{i}{ }_{j},
\end{aligned}
$$

where

$$
\begin{aligned}
& \Theta=\left(M_{a} g_{a}^{2} \frac{\partial}{\partial g_{a}^{2}}-h^{l m n} \frac{\partial}{\partial Y^{l m n}}\right) \\
& \left(\gamma_{1}\right)^{i}{ }_{j}=\Theta \gamma_{j}^{i} \\
& \triangle=2 \Theta \Theta^{*}+2 M_{a} M_{a}^{*} g_{a}^{2} \frac{\partial}{\partial g_{a}^{2}}+\widetilde{Y}_{l m n} \frac{\partial}{\partial Y_{l m n}}+\tilde{Y}^{l m n} \frac{\partial}{\partial Y^{l m n}}
\end{aligned}
$$

Here $M$ is the gaugino mass, $\tilde{Y}^{i j k}=\left(m^{2}\right)^{i}{ }_{l} Y^{j k l}+\left(m^{2}\right)^{j}{ }_{l} Y^{i k l}+\left(m^{2}\right)_{l}^{k} Y^{i j l}$, and

$$
X^{\mathrm{NSVZ}}=-2 \frac{g^{3}}{16 \pi^{2}} \frac{S}{\left[1-2 g^{2} C(G)\left(16 \pi^{2}\right)^{-1}\right]},
$$


where

$$
S=r^{-1} \operatorname{tr}\left[m^{2} C(R)\right]-M M^{*} C(G),
$$

$C(R), C(G)$ being the quadratic Casimirs for the matter and adjoint representations respectively and $X$ function above is given in the NSVZ scheme [18]. Eq. (11)holds in a class of renormalisation schemes that includes DRED' 19 , which we will use throughout. There is no corresponding exact form for $\mathrm{X}$ in the DRED' $[19$; however we only require here the leading contribution which is the same in both schemes; the subleading DRED' contribution is given in Ref [20]. These formulae can readily be specialised to the case of the RPV MSSM and their implementation can be automated.

\section{The R-parity violating MSSM scenario}

The unbrokrn $N=1$ theory is defined by the superpotential

$$
W=W_{1}+W_{2}
$$

where

$$
W_{1}=\epsilon_{a b}\left[\left(Y_{U}\right)_{i j} Q_{i}^{a x} H_{2}^{b} U_{j x}^{c}+\left(Y_{D}\right)_{i j} Q_{i}^{a x} H_{1}^{b} D_{j x}^{c}+\left(Y_{E}\right)_{i j} L_{i}^{a} H_{1}^{b} E_{j}^{c}-\mu H_{1}^{a} H_{2}^{b}\right],
$$

and

$$
\begin{aligned}
W_{2}= & \epsilon_{a b}\left[\frac{1}{2}\left(\Lambda_{E^{k}}\right)_{i j} L_{i}^{a} L_{j}^{b} E_{k}^{c}+\left(\Lambda_{D^{k}}\right)_{i j} L_{i}^{a} Q_{j}^{x b} D_{k x}^{c}\right]+\frac{1}{2} \epsilon_{x y z}\left(\Lambda_{U^{i}}\right)_{j k} U_{i}^{c x} D_{j}^{c y} D_{k}^{c z} \\
& \left.-\epsilon_{a b} \kappa^{i} L_{i}^{a} H_{2}^{b}\right) .
\end{aligned}
$$

In above equations, there is a summation over the generation indices $(i, j, k=1,2,3)$ and gauge indices $(x, y, z=1,2,3$ and $a, b=1,2) . \epsilon_{a b}$ and $\epsilon_{x y z}$ are totally antisymmetric tensors.

The soft SUSY breaking Lagrangian is given by:

$$
\begin{aligned}
-L_{\text {soft }}= & \left(m_{\widetilde{Q}^{2}}^{2}\right)_{i j} \widetilde{Q}_{i}^{\dagger} \widetilde{Q}_{j}+\left(m_{\widetilde{L}}^{2}\right)_{i j} \widetilde{L}_{i}^{\dagger} \widetilde{L}_{j}+\left(m_{\widetilde{U}^{c}}^{2}\right)_{i j} \widetilde{U}_{i}^{c} \widetilde{U}_{j}^{\dagger c}+\left(m_{\widetilde{D}^{c}}^{2}\right)_{i j} \widetilde{D}_{i}^{c} \widetilde{D}_{j}^{\dagger c} \\
& +\left(m_{\widetilde{E}^{c}}^{2}\right)_{i j} \widetilde{E}_{i}^{c} \widetilde{E}_{j}^{\dagger c}+m_{H_{2}}^{2} \hat{H}_{2}^{\dagger} \hat{H}_{2}+m_{H_{1}}^{2} \hat{H}_{1}^{\dagger} \hat{H}_{1}+\left[\left(h_{U}\right)_{i j} \hat{H}_{2} \widetilde{Q}_{i} \widetilde{U}_{j}^{c}\right. \\
& \left.+\left(h_{D}\right)_{i j} \hat{H}_{1} \widetilde{Q}_{i} \widetilde{D}_{j}^{c}+\left(h_{E}\right)_{i j} \hat{H}_{1} \widetilde{L}_{i} \widetilde{E}_{j}^{c}-B \hat{H}_{1} \hat{H}_{2}+h . c .\right] \\
& +\left[\frac{1}{2} M_{1} \widetilde{B} \widetilde{B}+\frac{1}{2} M_{2} \widetilde{W} \widetilde{W}+\frac{1}{2} M_{3} \widetilde{g} \widetilde{g}+h . c .\right]-L_{2},
\end{aligned}
$$

where we have suppressed $S U(2)$ indices. Here $\widetilde{B}, \widetilde{W}$ and $\widetilde{g}$ are the gaugino fields, $\widetilde{Q}, \widetilde{U}, \widetilde{D}$ and $\widetilde{L}, \widetilde{E}$ are the squark and slepton fields, respectively, and $\hat{H}_{1,2}$ are the $S U(2)$ doublet Higgs fields. $L_{2}$ includes terms which violate R-parity and it is given by:

$$
\begin{aligned}
-L_{2}= & \epsilon_{a b}\left[\frac{1}{2}\left(h_{E^{k}}\right)_{i j} \widetilde{L}_{i}^{a} \widetilde{L}_{j}^{b} \widetilde{E}_{k}^{c}+\left(h_{D^{k}}\right)_{i j} \widetilde{L}_{i}^{a} \widetilde{Q}_{j}^{x b} \widetilde{D}_{k x}^{c}+h . c\right] \\
& +\left[\frac{1}{2} \epsilon_{x y z}\left(h_{U^{i}}\right)_{j k} \widetilde{U}_{i}^{c x} \widetilde{D}_{j}^{c y} \widetilde{D}_{k}^{c z}-D_{i} \hat{H}_{2}^{a} \widetilde{L}_{i a}+h . c\right] \\
& +\widetilde{L}_{i a}^{\dagger}\left(m_{\tilde{L}_{i} H_{1}}^{2}\right) \hat{H}_{1}^{a}+\hat{H}_{1}^{\dagger a}\left(m_{H_{1} \tilde{L}_{i}}^{2}\right) \widetilde{L}_{i a}
\end{aligned}
$$




\section{Renormalization group equations for the R- parity violating MSSM scenario}

In In this paper we present the full two loop beta functions in the $R$-parity violating (RPV) MSSM scenario. Firstly, We demonstrate the results for the Yukawa coupling $\beta$-functions. In terms of the anomalous dimensions, the Yukawa $\beta$-functions are given by 13 :

$$
\begin{gathered}
\frac{d}{d t}\left(Y_{E}\right)_{i j}=\left(Y_{E}\right)_{i k} \gamma_{E_{k}}^{E_{j}}+\left(Y_{E}\right)_{i j} \gamma_{H_{1}}^{H_{1}}+\left(Y_{E}\right)_{k j} \gamma_{L_{k}}^{L_{i}}-\left(\Lambda_{E^{j}}\right)_{k i} \gamma_{L_{k}}^{H_{1}} \\
\frac{d}{d t}\left(Y_{D}\right)_{i j}=\left(Y_{D}\right)_{i k} \gamma_{D_{k}}^{D_{j}}+\left(Y_{D}\right)_{i j} \gamma_{H_{1}}^{H_{1}}+\left(Y_{D}\right)_{k j} \gamma_{Q_{k}}^{Q_{i}}-\left(\Lambda_{D^{j}}\right)_{k i} \gamma_{L_{k}}^{H_{1}} \\
\frac{d}{d t}\left(Y_{U}\right)_{i j}=\left(Y_{U}\right)_{i k} \gamma_{U_{k}}^{U_{j}}+\left(Y_{U}\right)_{i j} \gamma_{H_{2}}^{H_{2}}+\left(Y_{U}\right)_{k j} \gamma_{Q_{k}}^{Q_{i}} \\
\frac{d}{d t}\left(\Lambda_{E^{k}}\right)_{i j}=\left(\Lambda_{E^{l}}\right)_{i j} \gamma_{E_{l}}^{E_{k}}+\left(\Lambda_{E^{k}}\right)_{i l} \gamma_{L_{l}}^{L_{j}}+\left(Y_{E}\right)_{i k} \gamma_{H_{1}}^{L_{j}}-\left(\Lambda_{E^{k}}\right)_{j l} \gamma_{L_{l}}^{L_{i}}-\left(Y_{E}\right)_{j k} \gamma_{H_{1}}^{L_{i}} \\
\frac{d}{d t}\left(\Lambda_{D^{k}}\right)_{i j}=\left(\Lambda_{D^{l}}\right)_{i j} \gamma_{D_{l}}^{D_{k}}+\left(\Lambda_{D^{k}}\right)_{i l} \gamma_{Q_{l}}^{Q_{j}}+\left(\Lambda_{D^{k}}\right)_{l j} \gamma_{L_{l}}^{L_{i}}-\left(Y_{D}\right)_{j k} \gamma_{H_{1}}^{L_{i}} \\
\frac{d}{d t}\left(\Lambda_{U^{i}}\right)_{j k}=\left(\Lambda_{U^{i}}\right)_{j l} \gamma_{D_{l}}^{D_{k}}+\left(\Lambda_{U^{i}}\right)_{l k} \gamma_{D_{l}}^{D_{j}}+\left(\Lambda_{U^{l}}\right)_{j k} \gamma_{U_{l}}^{U_{i}}
\end{gathered}
$$

Here $t=\ln (Q)$ and $\gamma_{F_{j}}^{F_{i}}$ are the anomalous dimensions, and they are given by

$$
\gamma_{F_{j}}^{F_{i}}=\frac{1}{\left(16 \pi^{2}\right)} \gamma_{F_{j}}^{(1)}+\frac{1}{\left(16 \pi^{2}\right)^{2}} \gamma_{F_{j}}^{(2)}+\cdots
$$

where $\gamma^{(1)}, \gamma^{(2)}$ and $\cdots$ are 1 -loop, 2 -loop, $\cdots$ anomalous dimensions. The two-loop anomalous dimensions and gauge $\beta$-functions of the R-parity violating MSSM have been given in Ref [13]. Using the results of Ref [13] the two-loop Yukawa $\beta$-functions for the $R$-parity violating MSSM are given by:

$$
\begin{aligned}
& \left(16 \pi^{2}\right)^{2} \beta_{\left(Y_{E}\right)_{i j}}^{(2)}=\left(16 \pi^{2}\right)^{2} \beta_{\left(Y_{E}\right)_{R P C}}^{(2)} \\
& +\left(3 g_{2}^{2}-\frac{3}{5} g_{1}^{2}\right)\left(Y_{E}\right)_{i k} \operatorname{Tr}\left(\Lambda_{E^{j}} \Lambda_{E^{k}}^{\dagger}\right)-2\left(E \Lambda_{E^{l}} \Lambda_{E^{l}}^{\dagger} Y_{E}+3 E \Lambda_{D^{l}} \Lambda_{D^{l}}^{\dagger} Y_{E}\right)_{i j} \\
& +2\left(3 E \Lambda_{E^{j}} \Lambda_{D^{m}}^{*} Y_{D}-E \Lambda_{E^{j}} \Lambda_{E^{m}}^{\dagger} Y_{E}\right)_{i m} \\
& -2\left(Y_{E}\right)_{i k} \operatorname{Tr}\left[\Lambda_{E^{j}} \Lambda_{E^{k}}^{\dagger}\left(E+\Lambda_{E^{l}} \Lambda_{E^{l}}^{\dagger}+3 \Lambda_{D^{l}} \Lambda_{D^{l}}^{\dagger}\right)\right] \\
& +2\left(Y_{E}\right)_{i k}\left(3 Y_{D}^{\dagger} \Lambda_{D^{m}}^{t} \Lambda_{E^{k}}^{\dagger} Y_{E}-Y_{E}^{\dagger} \Lambda_{E^{m}} \Lambda_{E^{k}}^{\dagger} Y_{E}\right)_{m j}-\left(Y_{E}\right)_{i j}\left[(\tilde{E})_{n l} \operatorname{Tr}\left(\Lambda_{E^{l}}^{\dagger} \Lambda_{E^{n}}\right)\right] \\
& -\left(Y_{E}\right)_{i j}\left[6(\tilde{D})_{l p} \operatorname{Tr}\left(\Lambda_{D^{p}}^{\dagger} \Lambda_{D^{l}}\right)+\operatorname{Tr}\left(3 E \Lambda_{D^{q}} \Lambda_{D^{q}}^{\dagger}+E \Lambda_{E^{q}} \Lambda_{E^{q}}^{\dagger}+6 \tilde{D} \Lambda_{U^{q}}^{\dagger} \Lambda_{U^{q}}\right.\right. \\
& \left.\left.+3 D \Lambda_{D^{q}}^{t} \Lambda_{D^{q}}^{*}\right)\right]+\left(16 g_{3}^{2}-\frac{2}{5} g_{1}^{2}\right)\left(\Lambda_{D^{q}} \Lambda_{D^{q}}^{\dagger} Y_{E}\right)_{i j}+\frac{6}{5} g_{1}^{2}\left(\Lambda_{E^{q}} \Lambda_{E^{q}}^{\dagger} Y_{E}\right)_{i j} \\
& -(\tilde{E})_{m j}\left(Y_{E}\right)_{i l} \operatorname{Tr}\left(\Lambda_{E^{l}}^{\dagger} \Lambda_{E^{m}}\right)-2(\tilde{E})_{l m}\left(\Lambda_{E^{l}} \Lambda_{E^{m}}^{\dagger} Y_{E}\right)_{i j}-\left(\Lambda_{E^{l}} \Lambda_{E^{m}}^{\dagger} Y_{E}\right)_{i j} \operatorname{Tr}\left(\Lambda_{E^{l}}^{\dagger} \Lambda_{E^{m}}\right) \\
& -\left(Y_{E}\right)_{i m}\left(3 Y_{E}^{t} \Lambda_{E m}^{\dagger} \Lambda_{D^{p}} Y_{D}^{*}+Y_{E}^{t} \Lambda_{E^{m}}^{\dagger} \Lambda_{E p} Y_{E}^{*}\right)_{j p}+(\tilde{E})_{m j}\left(3 \Lambda_{E^{m}} \Lambda_{D^{P}}^{*} Y_{D}\right. \\
& \left.+\Lambda_{E^{m}} \Lambda_{E^{p}}^{*} Y_{E}\right)_{i p}-\left(Y_{E}^{t} \Lambda_{E^{m}}^{\dagger} E \Lambda_{E^{m}}+3 Y_{E}^{t} \Lambda_{E^{m}}^{\dagger} \Lambda_{D^{p}} \Lambda_{D^{p}}^{\dagger} \Lambda_{E^{m}}\right. \\
& \left.+Y_{E}^{t} \Lambda_{E^{m}}^{\dagger} \Lambda_{E^{p}} \Lambda_{E^{p}}^{\dagger} \Lambda_{E^{m}}\right)_{j i}-6\left(\Lambda_{E^{j}} \Lambda_{D^{n}}^{*} Y_{D} \Lambda_{U^{m}}^{\dagger} \Lambda_{U^{m}}\right)_{i n} \\
& -6\left(\Lambda_{D^{l}} \Lambda_{D^{m}}^{\dagger} Y_{E}\right)_{i j}\left[(\tilde{D})_{l m}+\operatorname{Tr}\left(\Lambda_{D^{l}}^{\dagger} \Lambda_{D^{m}}\right)+\left(\Lambda_{U^{q}} \Lambda_{U^{q}}^{\dagger}\right)_{m l}\right] \\
& -3\left(Y_{E}^{t} \Lambda_{D^{m}}^{*} D \Lambda_{D^{m}}^{t}+Y_{E}^{t} \Lambda_{D^{m}}^{*} T \Lambda_{D^{m}}^{t}\right)_{j i}-3\left(\Lambda_{D^{m}} \Lambda_{D^{p}}^{\dagger} \Lambda_{D^{p}} \Lambda_{D^{m}}^{\dagger} Y_{E}\right)_{i j}
\end{aligned}
$$




$$
\begin{aligned}
& +\left(\frac{2}{5} g_{1}^{2}-16 g_{3}^{2}\right)\left(\Lambda_{E^{j}} \Lambda_{D^{q}}^{*} Y_{D}\right)_{i q}-\frac{6}{5} g_{1}^{2}\left(\Lambda_{E^{j}} \Lambda_{E^{q}}^{*} Y_{E}\right)_{i q}-\left(3 \Lambda_{E^{j}} \Lambda_{E^{q}}^{\dagger} \Lambda_{D^{p}} \Lambda_{D^{p}}^{\dagger} Y_{E}\right)_{i q} \\
& -\left(\Lambda_{E^{j}} \Lambda_{E^{q}}^{\dagger} \Lambda_{E^{p}} \Lambda_{E^{p}}^{\dagger} Y_{E}+3 \Lambda_{E^{j}} \Lambda_{E^{q}}^{\dagger} E Y_{E}\right)_{i q}-\left(\Lambda_{E^{j}} \Lambda_{E^{n}}^{\dagger} Y_{E}\right)_{i l} \operatorname{Tr}\left(\Lambda_{E^{l}}^{\dagger} \Lambda_{E^{n}}\right) \\
& -3\left(\Lambda_{E^{j}} E^{*} \Lambda_{D^{m}}^{*} Y_{D}-\Lambda_{E^{j}} E^{*} \Lambda_{E^{m}}^{*} Y_{E}\right)_{i m}+9\left(\Lambda_{E^{j}} \Lambda_{D^{n}}^{*} D Y_{D}\right)_{i n} \\
& +6\left(\Lambda_{E^{j}} \Lambda_{D^{n}}^{*} Y_{D}\right)_{i l} \operatorname{Tr}\left(\Lambda_{D^{l}} \Lambda_{D^{n}}^{\dagger}\right)+3\left(\Lambda_{E^{j}} \Lambda_{D^{q}}^{*} T Y_{D}+3 \Lambda_{E^{j}} \Lambda_{D^{q}}^{*} \Lambda_{D^{m}}^{t} \Lambda_{D^{m}}^{*} Y_{D}\right)_{i q} . \\
& \left(16 \pi^{2}\right)^{2} \beta_{\left(Y_{D}\right)_{i j}}^{(2)}=\left(16 \pi^{2}\right)^{2} \beta_{\left(Y_{D}\right)_{R P C}}^{(2)}+\frac{6}{5} g_{1}^{2}\left(\Lambda_{D^{j}}^{t} \Lambda_{E^{q}}^{*} Y_{E}\right)_{i q}-\left(\frac{2}{5} g_{1}^{2}-16 g_{3}^{2}\right)\left(\Lambda_{D^{j}}^{t} \Lambda_{D^{q}}^{*} Y_{D}\right)_{i q} \\
& +\left(\Lambda_{D^{j}}^{t} \Lambda_{E^{q}}^{\dagger}\left[3 \Lambda_{D^{p}} \Lambda_{D^{p}}^{\dagger} Y_{E}+\Lambda_{E^{p}} \Lambda_{E^{p}}^{\dagger} Y_{E}+3 E Y_{E}\right)_{i q}\right. \\
& +\left(\Lambda_{D^{j}}^{t} \Lambda_{E^{n}}^{\dagger} Y_{E}\right)_{i l} \operatorname{Tr}\left(\Lambda_{E^{l}}^{\dagger} \Lambda_{E^{n}}\right)+3\left(\Lambda_{D^{j}}^{t} \tilde{E}\left[\Lambda_{D^{m}}^{*} Y_{D}-\Lambda_{E^{m}}^{*} Y_{E}\right]\right)_{i m} \\
& -\left(\Lambda_{D^{j}}^{t} \Lambda_{D^{n}}^{*}\left[9 D Y_{D}+6 Y_{D} \Lambda_{U^{m}}^{\dagger} \Lambda_{U^{m}}\right]\right)_{i n}+6 \operatorname{Tr}\left(\Lambda_{D^{l}} \Lambda_{D^{n}}^{\dagger}\right)\left(\Lambda_{D^{j}}^{t} \Lambda_{D^{n}}^{*} Y_{D}\right)_{i n} \\
& +3\left(\Lambda_{D^{j}}^{t} \Lambda_{D^{q}}^{*}\left(\left[T Y_{D}+\Lambda_{D^{m}}^{t} \Lambda_{D^{m}}^{*} Y_{D}\right]\right)_{i q}+\left(\frac{16}{3} g_{3}^{2}+\frac{16}{15} g_{1}^{2}\right)\left(\Lambda_{U^{q}} \Lambda_{U^{q}}^{\dagger} Y_{D}^{t}\right)_{j i}\right. \\
& +\left(6 g_{2}^{2}+\frac{2}{5} g_{1}^{2}\right)\left(Y_{D}\right)_{i k} \operatorname{Tr}\left(\Lambda_{D^{j}} \Lambda_{D^{k}}^{\dagger}\right)-2\left(Y_{D}^{t} \Lambda_{D^{p}}^{\dagger} \Lambda_{D^{p}} D^{t}\right)_{j i} \\
& -2\left(Y_{D}\right)_{i k} \operatorname{Tr}\left(\Lambda_{D^{j}}^{t} \Lambda_{D^{k}}^{*}[D+T]+\Lambda_{D^{k}}^{\dagger} \Lambda_{D^{j}} \Lambda_{D^{q}}^{\dagger} \Lambda_{D^{q}}\right) \\
& -\left(Y_{D}\right)_{i k} \operatorname{Tr}\left(6 \Lambda_{D^{q}} \Lambda_{D^{q}}^{\dagger} \Lambda_{D^{j}} \Lambda_{D^{k}}^{\dagger}+2 \Lambda_{E^{q}} \Lambda_{E^{q}}^{\dagger} \Lambda_{D^{j}} \Lambda_{D^{k}}^{\dagger}+2 E \Lambda_{D^{j}} \Lambda_{D^{k}}^{\dagger}\right) \\
& -4\left(Y_{D} \Lambda_{U^{m}}^{\dagger} \Lambda_{U^{p}} \Lambda_{U^{p}}^{\dagger} \Lambda_{U^{m}}\right)_{i j}+4\left(Y_{D} \Lambda_{U^{m}}^{\dagger}\right)_{i n}\left(\Lambda_{U^{m}}\right)_{j l} \operatorname{Tr}\left(\Lambda_{D^{l}} \Lambda_{D^{n}}^{\dagger}\right) \\
& -\left(6 D \Lambda_{D^{j}}^{t} \Lambda_{D^{p}}^{*} Y_{D}+2 D \Lambda_{D^{j}}^{t} \Lambda_{E^{q}}^{*} Y_{E}\right)_{i p}-\left(Y_{D}\right)_{i k}\left[6 Y_{D}^{t} \Lambda_{D^{k}}^{\dagger} \Lambda_{D^{p}} Y_{D}^{*}\right. \\
& \left.+2 Y_{D}^{t} \Lambda_{D^{j}}^{\dagger} \Lambda_{E^{p}} Y_{E}^{*}\right]_{j p}-2\left(Y_{D} \Lambda_{U^{n}}^{\dagger} \Lambda_{U^{l}}\right)_{i j}\left[\operatorname{Tr}\left(\Lambda_{U^{l}}^{\dagger} \Lambda_{U^{n}}\right)+2(\tilde{T})_{l n}\right] \\
& -\left(Y_{D}\right)_{i j} \operatorname{Tr}\left(3 E \Lambda_{D^{q}} \Lambda_{D^{q}}^{\dagger}+E \Lambda_{E^{q}} \Lambda_{E^{q}}^{\dagger}+6 \tilde{D} \Lambda_{U^{q}}^{\dagger} \Lambda_{U^{q}}+3 D \Lambda_{D^{q}}^{t} \Lambda_{D^{q}}^{*}\right. \\
& -\left(Y_{D}\right)_{i j} \tilde{E}_{n l} \operatorname{Tr}\left(\Lambda_{E^{l}}^{\dagger} \Lambda_{E^{n}}\right)-6\left(Y_{D}\right)_{i j} \tilde{D}_{l p} \operatorname{Tr}\left(\Lambda_{D^{p}}^{\dagger} \Lambda_{D^{l}}\right)+\frac{2}{5} g_{1}^{2}\left(Y_{D}^{t} \Lambda_{D^{q}}^{\dagger} \Lambda_{D^{q}}\right)_{j i} \\
& -2\left(Y_{D}^{t} \Lambda_{D^{l}}^{\dagger} \Lambda_{D^{m}}\right)_{j i}\left[\tilde{D}_{m l}+\left(\Lambda_{U^{q}} \Lambda_{U^{q}}^{\dagger}\right)_{l m}+\operatorname{Tr}\left(\Lambda_{D^{m}}^{\dagger} \Lambda_{D^{l}}\right)\right] \\
& -\left[Y_{D}^{t} \Lambda_{D^{m}}^{\dagger}\left(E \Lambda_{D^{m}}+3 \Lambda_{D^{q}} \Lambda_{D^{q}}^{\dagger} \Lambda_{D^{m}}+\Lambda_{E^{q}} \Lambda_{E^{q}}^{\dagger} \Lambda_{D^{m}}\right)\right]_{j i} \\
& -\left(Y_{D}\right)_{i l}\left[3 Y_{D}^{t} \Lambda_{D^{l}}^{\dagger} \Lambda_{D^{m}} Y_{D}^{*}+Y_{D}^{t} \Lambda_{D^{l}}^{\dagger} \Lambda_{E^{m}} Y_{E}^{*}\right]_{j m}-2\left(Y_{D} \Lambda_{U^{q}}^{\dagger} \Lambda_{U^{q}} \tilde{D}\right)_{i j} \\
& -2\left(Y_{D}\right)_{i m} \tilde{D}_{l j} \operatorname{Tr}\left(\Lambda_{D^{m}}^{\dagger} \Lambda_{D^{l}}\right)-\tilde{D}_{l j}\left(3 \Lambda_{D^{l}}^{t} \Lambda_{D^{m}}^{*} Y_{D}+\Lambda_{D^{l}}^{t} \Lambda_{E^{m}}^{*} Y_{E}\right)_{i m} \\
& -\left(Y_{U}\right)_{i l}\left(Y_{U}^{\dagger} Y_{D}\right)_{p j} \operatorname{Tr}\left(\Lambda_{U^{l}}^{\dagger} \Lambda_{U^{p}}\right)-4\left(\Lambda_{U^{m}} \tilde{D} \Lambda_{U^{m}}^{\dagger} Y_{D}^{t}\right)_{j i} \text {. } \\
& \left(16 \pi^{2}\right)^{2} \beta_{\left(Y_{U}\right)_{i j}}^{(2)}=\left(16 \pi^{2}\right)^{2} \beta_{\left(Y_{U}\right)_{R P C}}^{(2)}+\left(\frac{8}{3} g_{3}^{2}-\frac{4}{15} g_{1}^{2}\right)\left(Y_{U}\right)_{i k} \operatorname{Tr}\left(\Lambda_{U^{j}} \Lambda_{U^{k}}^{\dagger}\right)-2\left(T \Lambda_{D^{m}}^{t} \Lambda_{D^{m}}^{*} Y_{U}\right)_{i j} \\
& -4\left(Y_{U}\right)_{i k}\left[\left(\Lambda_{U^{k}}^{\dagger} \Lambda_{U^{j}}\right)_{l m} \operatorname{Tr}\left(\Lambda_{D^{m}}^{\dagger} \Lambda_{D^{l}}\right)+\operatorname{Tr}\left(\Lambda_{U^{k}}^{\dagger} \Lambda_{U^{j}} \tilde{D}\right)\right. \\
& \left.+\operatorname{Tr}\left(\Lambda_{U^{j}} \Lambda_{U^{k}}^{\dagger} \Lambda_{U^{p}} \Lambda_{U^{p}}^{\dagger}\right)\right]-\left(Y_{U}\right)_{i l}(\tilde{T})_{p j} \operatorname{Tr}\left(\Lambda_{U^{l}}^{\dagger} \Lambda_{U^{p}}\right) \\
& -3\left(Y_{U}\right)_{i j}\left[\operatorname{Tr}\left(\Lambda_{D^{q}}^{t} \Lambda_{D^{q}}^{*}\right)+\tilde{T}_{p m} \operatorname{Tr}\left(\Lambda_{U^{m}}^{\dagger} \Lambda_{U^{p}}\right)\right]+\frac{2}{5} g_{1}^{2}\left(Y_{U}^{t} \Lambda_{D^{q}}^{\dagger} \Lambda_{D^{q}}\right)_{j i} \\
& -2\left(Y_{U}^{\dagger} \Lambda_{D^{l}}^{\dagger} \Lambda_{D^{m}}\right)_{j i}\left[\tilde{D}_{m l}+\left(\Lambda_{U^{q}} \Lambda_{U^{q}}^{\dagger}\right)_{l m}+\operatorname{Tr}\left(\Lambda_{D^{m}}^{\dagger} \Lambda_{D^{l}}\right)\right] \\
& -\left(Y_{U}^{t} \Lambda_{D^{m}}^{\dagger} E \Lambda_{D^{m}}+3 Y_{U}^{t} \Lambda_{D^{m}}^{\dagger} \Lambda_{D^{q}} \Lambda_{D^{q}}^{\dagger} \Lambda_{D^{m}}+Y_{U}^{t} \Lambda_{D^{m}}^{\dagger} \Lambda_{E^{q}} \Lambda_{E^{q}}^{\dagger} \Lambda_{D^{m}}\right)_{j i} \\
& -\left(Y_{D}\right)_{i l}\left(3 Y_{U}^{t} \Lambda_{D^{l}}^{\dagger} \Lambda_{D^{m}} Y_{D}^{*}+Y_{U}^{t} \Lambda_{D^{l}}^{\dagger} \Lambda_{E^{m}} Y_{E}^{*}\right)_{j m} \\
& -2\left(Y_{D} \Lambda_{U^{q}}^{\dagger} \Lambda_{U^{q}} Y_{D}^{\dagger} Y_{U}\right)_{i j}-2\left(Y_{D}\right)_{i m}\left(Y_{D}^{\dagger} Y_{U}\right)_{l j} \operatorname{Tr}\left(\Lambda_{D^{m}}^{\dagger} \Lambda_{D^{l}}\right) \\
& -\left(Y_{D}^{\dagger} Y_{U}\right)_{l j}\left(3 \Lambda_{D^{l}}^{t} \Lambda_{D^{m}}^{*} Y_{D}+\Lambda_{D^{l}}^{t} \Lambda_{E^{m}}^{*} Y_{E}\right)_{i m} \text {. }
\end{aligned}
$$




$$
\begin{aligned}
& \left(16 \pi^{2}\right)^{2} \beta_{\left(\Lambda_{E^{k}}\right)_{i j}}^{(2)}=\left(\Lambda_{E^{l}}\right)_{i j}\left\{\left(6 g_{2}^{2}-\frac{6}{5} g_{1}^{2}\right)(\tilde{E})_{l k}+\left(3 g_{2}^{2}-\frac{3}{5} g_{1}^{2}\right) \operatorname{Tr}\left(\Lambda_{E^{k}} \Lambda_{E^{l}}^{\dagger}\right)\right. \\
& -2\left(Y_{E}^{\dagger} E Y_{E}+Y_{E}^{\dagger} \Lambda_{E^{q}} \Lambda_{E^{q}}^{\dagger} Y_{E}+3 Y_{E}^{\dagger} \Lambda_{D^{q}} \Lambda_{D^{q}}^{\dagger} Y_{E}\right)_{l k} \\
& -2(\tilde{E})_{l k} \operatorname{Tr}(E+3 D)+2\left(3 Y_{E}^{\dagger} \Lambda_{E^{k}} \Lambda_{D^{m}}^{*} Y_{D}\right. \\
& \left.-Y_{E}^{\dagger} \Lambda_{E^{k}} \Lambda_{E^{m}}^{\dagger} Y_{E}\right)_{l m}-2 \operatorname{Tr}\left[\Lambda _ { E ^ { k } } \Lambda _ { E ^ { l } } ^ { \dagger } \left(E+\Lambda_{E^{q}} \Lambda_{E^{q}}^{\dagger}\right.\right. \\
& \left.\left.\left.+3 \Lambda_{D^{q}} \Lambda_{D^{q}}^{\dagger}\right)\right]+2\left(3 Y_{D}^{\dagger} \Lambda_{D^{m}}^{t} \Lambda_{E^{l}}^{\dagger} Y_{E}-Y_{E}^{\dagger} \Lambda_{E^{m}} \Lambda_{E^{l}}^{\dagger} Y_{E}\right)_{m k}\right\} \\
& +\left(\Lambda_{E^{k}}\right)_{i l}\left\{\left(16 g_{3}^{2}-\frac{2}{5} g_{1}^{2}\right)\left(\Lambda_{D^{q}} \Lambda_{D^{q}}^{\dagger}\right)_{j l}-2\left(E^{2}\right)_{j l}\right. \\
& +\frac{6}{5} g_{1}^{2}\left(E+\Lambda_{E^{q}} \Lambda_{E^{q}}^{\dagger}\right)_{j l}+\left(Y_{E}^{*}\right)_{l m}\left(3 \Lambda_{E^{m}} \Lambda_{D^{p}}^{*} Y_{D}+\Lambda_{E^{m}} \Lambda_{E^{p}}^{*} Y_{E}\right)_{j p} \\
& -\left(Y_{E}^{\dagger}\right)_{m l}\left(Y_{E}\right)_{j n} \operatorname{Tr}\left(\Lambda_{E^{n}}^{\dagger} \Lambda_{E^{m}}\right)-2\left(\Lambda_{E^{n}} \Lambda_{E^{m}}^{\dagger}\right)_{j l}(\tilde{E})_{n m} \\
& -\left(\Lambda_{E^{n}} \Lambda_{E^{m}}^{\dagger}\right)_{j l} \operatorname{Tr}\left(\Lambda_{E^{n}}^{\dagger} \Lambda_{E^{m}}\right)-(E)_{j l} \operatorname{Tr}(E+3 D) \\
& -\left(Y_{E}\right)_{j m}\left(3 \Lambda_{E^{m}}^{\dagger} \Lambda_{D^{p}} Y_{D}^{*}+\Lambda_{E}^{\dagger} \Lambda_{E^{p}} Y_{E}^{*}\right)_{l p} \\
& -\left(\Lambda_{E^{m}}^{\dagger} E \Lambda_{E^{m}}+3 \Lambda_{E^{m}}^{\dagger} \Lambda_{D^{p}} \Lambda_{D^{p}}^{\dagger} \Lambda_{E^{m}}+\Lambda_{E^{m}}^{\dagger} \Lambda_{E^{p}} \Lambda_{E^{p}}^{\dagger} \Lambda_{E^{m}}\right)_{l j} \\
& -6\left(\Lambda_{D^{n}} \Lambda_{D^{m}}^{\dagger}\right)_{j l}\left[(\tilde{D})_{n m}+\operatorname{Tr}\left(\Lambda_{D^{n}}^{\dagger} \Lambda_{D^{m}}\right)+\left(\Lambda_{U^{q}} \Lambda_{U^{q}}^{\dagger}\right)_{m n}\right] \\
& \left.-3\left(\Lambda_{D^{m}}^{*} D \Lambda_{D^{m}}^{t}+\Lambda_{D^{m}}^{*} T \Lambda_{D^{m}}^{t}\right)_{l j}-3\left(\Lambda_{D^{m}} \Lambda_{D^{p}}^{\dagger} \Lambda_{D^{p}} \Lambda_{D^{m}}^{\dagger}\right)_{j l}\right\} \\
& -\left(\Lambda_{E^{k}}\right)_{j l}\left\{\left(16 g_{3}^{2}-\frac{2}{5} g_{1}^{2}\right)\left(\Lambda_{D^{q}} \Lambda_{D^{q}}^{\dagger}\right)_{i l}+\frac{6}{5} g_{1}^{2}\left(E+\Lambda_{E^{q}} \Lambda_{E^{q}}^{\dagger}\right)_{i l}\right. \\
& -2\left(E^{2}\right)_{i l}-\left(Y_{E}^{\dagger}\right)_{m l}\left(Y_{E}\right)_{i n} \operatorname{Tr}\left(\Lambda_{E^{n}}^{\dagger} \Lambda_{E^{m}}\right)-2\left(\Lambda_{E^{n}} \Lambda_{E^{m}}^{\dagger}\right)_{i l}(\tilde{E})_{n m} \\
& -\left(\Lambda_{E^{n}} \Lambda_{E^{m}}^{\dagger}\right)_{i l} \operatorname{Tr}\left(\Lambda_{E^{n}}^{\dagger} \Lambda_{E^{m}}\right)-E_{i l} \operatorname{Tr}(E+3 D)+\left(Y_{E}^{*}\right)_{l m}\left(3 \Lambda_{E^{m}} \Lambda_{D^{p}}^{*} Y_{D}\right. \\
& \left.+\Lambda_{E^{m}} \Lambda_{E^{p}}^{*} Y_{E}\right)_{i p}-\left(\Lambda_{E^{m}}^{\dagger} E \Lambda_{E^{m}}+3 \Lambda_{E m}^{\dagger} \Lambda_{D^{p}} \Lambda_{D^{p}}^{\dagger} \Lambda_{E^{m}}\right. \\
& \left.+\Lambda_{E^{m}}^{\dagger} \Lambda_{E^{p}} \Lambda_{E^{p}}^{\dagger} \Lambda_{E^{m}}\right)_{l i}-\left(Y_{E}\right)_{i m}\left(3 \Lambda_{E^{m}}^{\dagger} \Lambda_{D^{p}} Y_{D}^{*}+\Lambda_{E^{m}}^{\dagger} \Lambda_{E^{p}} Y_{E}^{*}\right)_{l p} \\
& -6\left(\Lambda_{D^{n}} \Lambda_{D^{m}}^{\dagger}\right)_{i l}\left[(\tilde{D})_{n m}+\operatorname{Tr}\left(\Lambda_{D^{n}}^{\dagger} \Lambda_{D^{m}}\right)+\left(\Lambda_{U^{q}} \Lambda_{U^{q}}^{\dagger}\right)_{m n}\right] \\
& \left.-3\left(\Lambda_{D^{m}}^{*} D \Lambda_{D^{m}}^{t}+\Lambda_{D^{m}}^{*} T \Lambda_{D^{m}}^{t}\right)_{l i}-3\left(\Lambda_{D^{m}} \Lambda_{D^{p}}^{\dagger} \Lambda_{D^{p}} \Lambda_{D^{m}}^{\dagger}\right)_{i l}\right\} \\
& +\left(Y_{E}\right)_{i k}\left\{\left(\frac{2}{5} g_{1}^{2}-16 g_{3}^{2}\right)\left(\Lambda_{D^{q}} Y_{D}^{*}\right)_{j p}-\frac{6}{5} g_{1}^{2}\left(\Lambda_{E} q Y_{E}^{*}\right)_{j p}\right. \\
& -\left(3 \Lambda_{E^{q}}^{t} \Lambda_{D^{p}}^{*} \Lambda_{D^{p}}^{t} Y_{E}^{*}+\Lambda_{E^{q}}^{t} \Lambda_{E^{p}}^{*} \Lambda_{E^{p}}^{t} Y_{E}^{*}+3 \Lambda_{E^{q}}^{t} E^{t} Y_{E}^{*}\right)_{j q} \\
& -\left(\Lambda_{E^{n}}^{t} Y_{E}^{*}\right)_{j l} \operatorname{Tr}\left(\Lambda_{E^{l}}^{t} \Lambda_{E^{n}}^{*}\right)-6\left(\Lambda_{D^{n}} Y_{D}^{*} \Lambda_{U^{m}}^{t} \Lambda_{U^{m}}^{*}\right)_{j n} \\
& -3\left(E \Lambda_{D^{m}} Y_{D}^{*}-E \Lambda_{E^{m}} Y_{E}^{*}\right)_{j m}+9\left(\Lambda_{D^{n}} D^{t} Y_{D}^{*}\right)_{j n} \\
& \left.+6\left(\Lambda_{D^{n}} Y_{D}^{*}\right)_{j l} \operatorname{Tr}\left(\Lambda_{D^{l}}^{*} \Lambda_{D^{n}}^{t}\right)+3\left(Y_{D}^{\dagger} T \Lambda_{D^{q}}^{t}\right)_{q j}+3\left(\Lambda_{D^{q}} \Lambda_{D^{m}}^{\dagger} \Lambda_{D^{m}} Y_{D}^{*}\right)_{j q}\right\} \\
& -\left(Y_{E}\right)_{j k}\left\{\left(\frac{2}{5} g_{1}^{2}-16 g_{3}^{2}\right)\left(\Lambda_{D^{q}} Y_{D}^{*}\right)_{i q}-\frac{6}{5} g_{1}^{2}\left(\Lambda_{E q} Y_{E}^{*}\right)_{i q}\right. \\
& -\left(3 \Lambda_{E q}^{t} \Lambda_{D^{p}}^{*} \Lambda_{D^{p}}^{t} Y_{E}^{*}+\Lambda_{E q}^{t} \Lambda_{E p}^{*} \Lambda_{E p}^{t} Y_{E}^{*}+3 \Lambda_{E q}^{t} E^{t} Y_{E}^{*}\right)_{i q} \\
& -\left(\Lambda_{E^{n}}^{t} Y_{E}^{*}\right)_{i l} \operatorname{Tr}\left(\Lambda_{E^{l}}^{t} \Lambda_{E^{n}}^{*}\right)-3\left(E \Lambda_{D^{m}} Y_{D}^{*}-E \Lambda_{E^{m}} Y_{E}^{*}\right)_{i m} \\
& +9\left(\Lambda_{D^{n}} D^{t} Y_{D}^{*}\right)_{i n}-6\left(\Lambda_{D^{n}} Y_{D^{*}}^{*} \Lambda_{U^{m}}^{t} \Lambda_{U^{m}}^{*}\right)_{i n}+6\left(\Lambda_{D^{n}} Y_{D}^{*}\right)_{i l} \operatorname{Tr}\left(\Lambda_{D^{l}}^{*} \Lambda_{D^{n}}^{t}\right) \\
& \left.+3\left(\Lambda_{D^{q}} Y_{U}^{*} Y_{U}^{t} Y_{D}^{*}\right)_{i q}+3\left(\Lambda_{D^{q}} \Lambda_{D^{m}}^{\dagger} \Lambda_{D^{m}} Y_{D}^{*}\right)_{i q}\right\} \\
& +\left(\Lambda_{E^{k}}\right)_{i j}\left[\frac{27}{2} g_{1}^{4}+\frac{15}{2} g_{2}^{4}+\frac{9}{5} g_{2}^{2} g_{1}^{2}\right] \\
& \left(16 \pi^{2}\right)^{2} \beta_{\left(\Lambda_{D^{k}}\right)_{i j}}^{(2)}=\left(\Lambda_{D^{l}}\right)_{i j}\left\{\left(\frac{16}{3} g_{3}^{2}+\frac{16}{15} g_{1}^{2}\right)\left(\Lambda_{U^{q}} \Lambda_{U^{q}}^{\dagger}\right)_{k l}+\left(6 g_{2}^{2}+\frac{2}{5} g_{1}^{2}\right)\left[D_{k l}+\operatorname{Tr}\left(\Lambda_{D^{k}} \Lambda_{D^{l}}^{\dagger}\right)\right]\right. \\
& -2\left(Y_{D}^{\dagger} D Y_{D}+Y_{D}^{\dagger} T Y_{D}\right)_{l k}-\left(6 Y_{D}^{\dagger} \Lambda_{D^{k}}^{t} \Lambda_{D^{p}}^{*} Y_{D}\right)
\end{aligned}
$$




$$
\begin{aligned}
& -2(\tilde{D})_{l k} \operatorname{Tr}(E+3 D)+4\left(\Lambda_{U^{m}}^{\dagger}\right)_{l n}\left(\Lambda_{U^{m}}\right)_{k h} \operatorname{Tr}\left(\Lambda_{D^{h}} \Lambda_{D^{n}}^{\dagger}\right) \\
& -\left(2 Y_{D}^{t} \Lambda_{D^{p}}^{\dagger} \Lambda_{D^{p}} Y_{D}^{*}+4 \Lambda_{U^{m}} \tilde{D} \Lambda_{U^{m}}^{\dagger}\right)_{k l}-4\left(\Lambda_{U^{m}}^{\dagger} \Lambda_{U^{p}} \Lambda_{U^{p}}^{\dagger} \Lambda_{U^{m}}\right)_{l k} \\
& -2 \operatorname{Tr}\left(\Lambda_{D^{k}}^{t} \Lambda_{D^{l}}^{*}(D+T)+\Lambda_{D^{l}}^{\dagger} \Lambda_{D^{k}} \Lambda_{D^{q}}^{\dagger} \Lambda_{D^{q}}\right) \\
& -\operatorname{Tr}\left(6 \Lambda_{D^{q}} \Lambda_{D^{q}}^{\dagger} \Lambda_{D^{k}} \Lambda_{D^{l}}^{\dagger}+2 \Lambda_{E^{q}} \Lambda_{E^{q}}^{\dagger} \Lambda_{D^{k}} \Lambda_{D^{l}}^{\dagger}+2 E \Lambda_{D^{k}} \Lambda_{D^{l}}^{\dagger}\right) \\
& -2\left(Y_{D}^{\dagger} \Lambda_{D^{k}}^{t} \Lambda_{E^{p}}^{*} Y_{E}\right)_{l p}-\left(6 Y_{D}^{t} \Lambda_{D^{l}}^{\dagger} \Lambda_{D^{p}} Y_{D}^{*}+2 Y_{D}^{t} \Lambda_{D^{k}}^{\dagger} \Lambda_{E^{p}} Y_{E}^{*}\right)_{k p} \\
& \left.-2\left(\Lambda_{U^{n}}^{\dagger} \Lambda_{U^{h}}\right)_{l k}\left[\operatorname{Tr}\left(\Lambda_{U^{h}}^{\dagger} \Lambda_{U^{n}}\right)+2(\tilde{T})_{h n}\right]\right\} \\
& +\frac{2}{5} g_{1}^{2}\left[\left(D \Lambda_{D^{k}}^{t}+2 T \Lambda_{D^{k}}^{t}\right)_{j i}+\left(\Lambda_{D^{k}} \Lambda_{D^{q}}^{\dagger} \Lambda_{D^{q}}\right)_{i j}\right]-2\left(D^{2} \Lambda_{D^{k}}^{t}\right)_{j i} \\
& -\left(D \Lambda_{D^{k}}^{t}\right)_{j i} \operatorname{Tr}(E+3 D)-2\left(T^{2} \Lambda_{D^{k}}^{t}\right)_{j i}-3\left(T \Lambda_{D^{k}}^{t}\right)_{j i} \operatorname{Tr}(T) \\
& -2\left(\Lambda_{D^{k}} \Lambda_{D^{p}}^{\dagger} \Lambda_{D^{m}}\right)_{i j}\left[(\tilde{D})_{m p}+\left(\Lambda_{U^{q}} \Lambda_{U^{q}}^{\dagger}\right)_{p m}+\operatorname{Tr}\left(\Lambda_{D^{m}}^{\dagger} \Lambda_{D^{p}}\right)\right] \\
& -\left[\Lambda_{D^{k}} \Lambda_{D^{m}}^{\dagger}\left(E \Lambda_{D^{m}}+3 \Lambda_{D^{q}} \Lambda_{D^{q}}^{\dagger} \Lambda_{D^{m}}+\Lambda_{E^{q}} \Lambda_{E^{q}}^{\dagger} \Lambda_{D^{m}}\right)\right]_{i j} \\
& -\left(Y_{D}\right)_{j p}\left(3 \Lambda_{D^{k}} \Lambda_{D^{p}}^{\dagger} \Lambda_{D^{m}} Y_{D}^{*}+\Lambda_{D^{k}} \Lambda_{D^{p}}^{\dagger} \Lambda_{E^{m}} Y_{E}^{*}\right)_{i m}-2\left(Y_{D} \Lambda_{U^{q}}^{\dagger} \Lambda_{U^{q}} Y_{D^{\dagger}}^{\dagger} \Lambda_{D^{k}}^{t}\right)_{j i} \\
& -2\left(Y_{D}\right)_{j m}\left(Y_{D^{\dagger}}^{\dagger} \Lambda_{D^{k}}^{t}\right)_{p i} \operatorname{Tr}\left(\Lambda_{D^{m}}^{\dagger} \Lambda_{D^{p}}\right)-\left(Y_{U}\right)_{j p}\left(Y_{U}^{\dagger} \Lambda_{D^{k}}^{t}\right)_{n i} \operatorname{Tr}\left(\Lambda_{U^{p}}^{\dagger} \Lambda_{U^{n}}\right) \\
& -\left(\Lambda_{D^{k}} Y_{D}^{*}\right)_{i p}\left(3 \Lambda_{D^{p}}^{t} \Lambda_{D^{m}}^{*} Y_{D}+\Lambda_{D^{p}}^{t} \Lambda_{E^{m}}^{*} Y_{E}\right)_{j m} \\
& +\left(Y_{D}\right)_{j k}\left\{-\left(\frac{2}{5} g_{1}^{2}-16 g_{3}^{2}\right)\left(\Lambda_{D^{q}} Y_{D}^{*}\right)_{i q}+\frac{6}{5} g_{1}^{2}\left(\Lambda_{E^{q}} Y_{E}^{*}\right)_{i q}\right. \\
& +\left(3 \Lambda_{E q}^{t} \Lambda_{D^{p}}^{*} \Lambda_{D^{p}}^{t} Y_{E}^{*}+\Lambda_{E^{q}}^{t} \Lambda_{E^{p}}^{*} \Lambda_{E^{p}}^{t} Y_{E}^{*}+3 \Lambda_{E^{q}}^{t} E^{*} Y_{E}^{*}\right. \\
& \left.-3 \Lambda_{D^{q}} Y_{U}^{*} Y_{U}^{t} Y_{D}^{*}-3 \Lambda_{D^{q}} \Lambda_{D^{m}}^{\dagger} \Lambda_{D^{m}} Y_{D}^{*}\right)_{i q}+\left(\Lambda_{E^{n}}^{t} Y_{E}^{*}\right)_{i l} \operatorname{Tr}\left(\Lambda_{E^{l}}^{t} \Lambda_{E^{n}}^{*}\right) \\
& +3\left[E \Lambda_{D^{m}} Y_{D}^{*}-E \Lambda_{E^{m}} Y_{E}^{*}\right]_{i m}-\left[9 \Lambda_{D^{n}} D^{t} Y_{D}^{*}\right. \\
& \left.\left.-6 \Lambda_{D^{n}} Y_{D}^{*} \Lambda_{U^{m}}^{t} \Lambda_{U^{m}}^{*}\right]_{i n}-6\left(\Lambda_{D^{n}} Y_{D}^{*}\right)_{i l} \operatorname{Tr}\left(\Lambda_{D^{l}}^{*} \Lambda_{D^{n}}^{t}\right)\right\} \\
& +\left(\Lambda_{D^{k}}\right)_{i j}\left[\frac{287}{90} g_{1}^{4}-\frac{16}{9} g_{3}^{4}+\frac{15}{2} g_{2}^{4}+g_{1}^{2} g_{2}^{2}+8 g_{3}^{2} g_{2}^{2}+\frac{8}{9} g_{3}^{2} g_{1}^{2}\right] \\
& +\left(\Lambda_{D^{q}} \Lambda_{D^{q}}^{\dagger} \Lambda_{D^{k}}\right)_{i j}\left(16 g_{3}^{2}-\frac{2}{5} g_{1}^{2}\right)+\frac{6}{5} g_{1}^{2}\left(E \Lambda_{D^{k}}\right. \\
& \left.+\Lambda_{E q} \Lambda_{E^{q}}^{\dagger} \Lambda_{D^{k}}\right)_{i j}-\left(2 E^{2} \Lambda_{D^{k}}-3 \Lambda_{D^{n}} \Lambda_{D^{p}}^{\dagger} \Lambda_{D^{p}} \Lambda_{D^{n}}^{\dagger} \Lambda_{D^{k}}\right)_{i j} \\
& -\left(Y_{E}^{\dagger} \Lambda_{D^{k}}\right)_{n j}\left(Y_{E}\right)_{i m} \operatorname{Tr}\left(\Lambda_{E^{m}}^{\dagger} \Lambda_{E^{n}}\right)-\left(\Lambda_{E^{m}} \Lambda_{E^{n}}^{\dagger} \Lambda_{D^{k}}\right)_{i j}\left[(2 \tilde{E})_{m n}\right. \\
& \left.+\operatorname{Tr}\left(\Lambda_{E^{m}}^{\dagger} \Lambda_{E^{n}}\right)\right]-\left(E \Lambda_{D^{k}}\right)_{i j} \operatorname{Tr}(E+3 D) \\
& -\left(Y_{E}\right)_{i n}\left(3 \Lambda_{D^{k}}^{t} \Lambda_{E^{n}}^{\dagger} \Lambda_{D^{p}} Y_{D}^{*}+\Lambda_{D^{k}}^{t} \Lambda_{E^{n}}^{\dagger} \Lambda_{E^{p}} Y_{E}^{*}\right)_{j p}+\left(Y_{E^{\prime}}^{\dagger} \Lambda_{D^{k}}\right)_{n j}\left(3 \Lambda_{E^{n}} \Lambda_{D^{p}}^{*} Y_{D}\right. \\
& \left.+\Lambda_{E^{n}} \Lambda_{E^{p}}^{*} Y_{E}\right)_{i p}-\left(\Lambda_{D^{k}}^{t} \Lambda_{E^{n}}^{\dagger} E \Lambda_{E^{n}}+3 \Lambda_{D^{k}}^{t} \Lambda_{E^{n}}^{\dagger} \Lambda_{D^{p}} \Lambda_{D^{p}}^{\dagger} \Lambda_{E^{n}}\right. \\
& \left.+\Lambda_{D^{k}}^{t} \Lambda_{E^{n}}^{\dagger} \Lambda_{E^{p}} \Lambda_{E^{p}}^{\dagger} \Lambda_{E^{n}}\right)_{j i}-6\left(\Lambda_{D^{m}} \Lambda_{D^{n}}^{\dagger} \Lambda_{D^{k}}\right)_{i j}\left[(\tilde{D})_{m n}+\operatorname{Tr}\left(\Lambda_{D^{m}}^{\dagger} \Lambda_{D^{n}}\right)\right. \\
& \left.+\left(\Lambda_{U^{q}} \Lambda_{U^{q}}^{\dagger}\right)_{n m}\right]-3\left(\Lambda_{D^{k}}^{t} \Lambda_{D^{n}}^{*} D \Lambda_{D^{n}}^{t}+\Lambda_{D^{k}}^{t} \Lambda_{D^{n}}^{*} T \Lambda_{D^{n}}^{t}\right)_{j i} \\
& \left(16 \pi^{2}\right)^{2} \beta_{\left(\Lambda_{U^{i}}\right)_{j k}}^{(2)}=-\left(\frac{16}{3} g_{3}^{2}+\frac{16}{15} g_{1}^{2}\right)\left(\Lambda_{U^{q}} \Lambda_{U^{q}}^{\dagger} \Lambda_{U^{i}}\right)_{k j}+\left(6 g_{2}^{2}+\frac{2}{5} g_{1}^{2}\right)\left(\Lambda_{U^{i}}\right)_{j l} \operatorname{Tr}\left(\Lambda_{D^{k}} \Lambda_{D^{l}}^{\dagger}\right) \\
& -\left(6 g_{2}^{2}+\frac{2}{5} g_{1}^{2}\right)\left(D \Lambda_{U^{i}}\right)_{k j}-2\left(\Lambda_{U^{i}} Y_{D}^{\dagger} D Y_{D}\right)_{j k}-2\left(\Lambda_{U^{i}} Y_{D}^{\dagger} T Y_{D}\right)_{j k} \\
& +2 \operatorname{Tr}(E+3 D)\left[\left(\Lambda_{U^{i}} \tilde{D}\right)_{k j}-\left(\Lambda_{U^{i}} \tilde{D}\right)_{j k}\right]+2\left(Y_{D}^{t} \Lambda_{D^{p}}^{\dagger} \Lambda_{D^{p}} Y_{D}^{*} \Lambda_{U^{i}}\right)_{k j} \\
& -2\left(\Lambda_{U^{i}}\right)_{j l} \operatorname{Tr}\left(\Lambda_{D^{k}}^{t} \Lambda_{D^{l}}^{*}(D+T)+\Lambda_{D^{l}}^{\dagger} \Lambda_{D^{k}} \Lambda_{D^{q}}^{\dagger} \Lambda_{D^{q}}\right) \\
& -\left(\Lambda_{U^{i}}\right)_{j l} \operatorname{Tr}\left(6 \Lambda_{D^{q}} \Lambda_{D^{q}}^{\dagger} \Lambda_{D^{k}} \Lambda_{D^{l}}^{\dagger}+2 \Lambda_{E^{q}} \Lambda_{E^{q}}^{\dagger} \Lambda_{D^{k}} \Lambda_{D^{l}}^{\dagger}+2 E \Lambda_{D^{k}} \Lambda_{D^{l}}^{\dagger}\right) \\
& -\left(6 \Lambda_{U^{i}} Y_{D}^{\dagger} \Lambda_{D^{k}}^{t} \Lambda_{D^{p}}^{*} Y_{D}+\Lambda_{U^{i}} Y_{D}^{\dagger} \Lambda_{D^{k}}^{t} \Lambda_{E^{p}}^{*} Y_{E}\right)_{j p} \\
& +4\left(\Lambda_{U^{m}} \tilde{D} \Lambda_{U^{m}}^{\dagger} \Lambda_{U^{i}}\right)_{k j}-4\left(\Lambda_{U^{i}} \Lambda_{U^{m}}^{\dagger}\right)_{k n}\left(\Lambda_{U^{m}}\right)_{j h} \operatorname{Tr}\left(\Lambda_{D^{h}} \Lambda_{D^{n}}^{\dagger}\right)
\end{aligned}
$$




$$
\begin{aligned}
& +4\left(\Lambda_{U^{i}} \Lambda_{U^{m}}^{\dagger}\right)_{j n}\left(\Lambda_{U^{m}}\right)_{k h} \operatorname{Tr}\left(\Lambda_{D^{h}} \Lambda_{D^{n}}^{\dagger}\right)-4\left(\Lambda_{U^{i}} \Lambda_{U^{m}}^{\dagger} \Lambda_{U^{p}} \Lambda_{U^{p}}^{\dagger} \Lambda_{U^{m}}\right)_{j k} \\
& -2\left(\Lambda_{U^{i}} \Lambda_{U^{n}}^{\dagger} \Lambda_{U^{h}}\right)_{j k}\left(\operatorname{Tr}\left[\Lambda_{U^{h}}^{\dagger} \Lambda_{U^{n}}\right]+2 \tilde{T}_{h n}\right)-\left(\Lambda_{U^{i}}\right)_{j l}\left(6 Y_{D}^{t} \Lambda_{D^{l}}^{\dagger} \Lambda_{D^{p}} Y_{D}^{*}\right. \\
& \left.+2 Y_{D}^{t} \Lambda_{D^{k}}^{\dagger} \Lambda_{E^{p}} Y_{E}^{*}\right)_{k p}+\left(\frac{16}{3} g_{3}^{2}+\frac{16}{15} g_{1}^{2}\right)\left(\Lambda_{U^{q}} \Lambda_{U^{q}}^{\dagger} \Lambda_{U^{i}}\right)_{j k} \\
& +\left(6 g_{2}^{2}+\frac{2}{5} g_{1}^{2}\right)\left[\left(D \Lambda_{U^{i}}\right)_{j k}+\operatorname{Tr}\left(\Lambda_{D^{j}} \Lambda_{D^{l}}^{\dagger}\right)\left(\Lambda_{U^{i}}\right)_{l k}\right] \\
& +2\left(\Lambda_{U^{i}} Y_{D}^{\dagger} D Y_{D}\right)_{k j}+2\left(\Lambda_{U^{i}} Y_{D}^{\dagger} T Y_{D}\right)_{k j}-2\left(Y_{D}^{t} \Lambda_{D^{p}}^{\dagger} \Lambda_{D^{p}} Y_{D}^{*} \Lambda_{U^{i}}\right)_{j k} \\
& -2\left(\Lambda_{U^{i}}\right)_{l k} \operatorname{Tr}\left(\Lambda_{D^{j}}^{t} \Lambda_{D^{l}}^{*}(D+T)+\Lambda_{D^{l}}^{\dagger} \Lambda_{D^{j}} \Lambda_{D^{q}}^{\dagger} \Lambda_{D^{q}}\right) \\
& -\left(\Lambda_{U^{i}}\right)_{l k} \operatorname{Tr}\left(6 \Lambda_{D^{q}} \Lambda_{D^{q}}^{\dagger} \Lambda_{D^{j}} \Lambda_{D^{l}}^{\dagger}+2 \Lambda_{E^{q}} \Lambda_{E^{q}}^{\dagger} \Lambda_{D^{j}} \Lambda_{D^{l}}^{\dagger}+2 E \Lambda_{D^{j}} \Lambda_{D^{l}}^{\dagger}\right) \\
& -4\left(\Lambda_{U^{m}} \tilde{D} \Lambda_{U^{m}}^{\dagger} \Lambda_{U^{i}}\right)_{j k}+4\left(\Lambda_{U^{i}} \Lambda_{U^{m}}^{\dagger} \Lambda_{U^{p}} \Lambda_{U^{p}}^{\dagger} \Lambda_{U^{m}}\right)_{k j} \\
& +2\left(\Lambda_{U^{i}} Y_{D^{\dagger}}^{\dagger} \Lambda_{D^{j}}^{t} \Lambda_{E^{p}}^{*} Y_{E}\right)_{k p}-\left(\Lambda_{U^{i}}\right)_{l k}\left(6 Y_{D}^{t} \Lambda_{D^{l}}^{\dagger} \Lambda_{D^{p}} Y_{D}^{*}\right. \\
& \left.+2 Y_{D}^{t} \Lambda_{D^{j}}^{\dagger} \Lambda_{E^{p}} Y_{E}^{*}\right)_{j p}+2\left(\Lambda_{U^{i}} \Lambda_{U^{n}}^{\dagger} \Lambda_{U^{h}}\right)_{k j}\left[\operatorname{Tr}\left(\Lambda_{U^{h}}^{\dagger} \Lambda_{U^{n}}\right)+2(\tilde{T})_{h n}\right] \\
& +\left(6 \Lambda_{U^{i}} Y_{D^{\dagger}}^{\dagger} \Lambda_{D^{j}}^{t} \Lambda_{D^{p}}^{*} Y_{D}\right)_{k p}+\left(\Lambda_{U^{l}}\right)_{j k}\left\{\left(\frac{8}{3} g_{3}^{2}-\frac{4}{15} g_{1}^{2}\right) \operatorname{Tr}\left(\Lambda_{U^{i}} \Lambda_{U^{l}}^{\dagger}\right)\right. \\
& +\left(6 g_{2}^{2}-\frac{2}{5} g_{1}^{2}\right)(\tilde{T})_{l i}-2\left(Y_{U}^{\dagger} T Y_{U}+Y_{U}^{\dagger} D Y_{U}+Y_{U}^{\dagger} \Lambda_{D^{m}}^{t} \Lambda_{D^{m}}^{*} Y_{U}\right)_{l i} \\
& -6 \tilde{T}_{l i} \operatorname{Tr}(T)-4\left(\Lambda_{U^{l}}^{\dagger} \Lambda_{U^{i}}\right)_{h m} \operatorname{Tr}\left(\Lambda_{D^{m}}^{\dagger} \Lambda_{D^{h}}\right)-4 \operatorname{Tr}\left(\Lambda_{U^{i}} \Lambda_{U^{l}}^{\dagger} \Lambda_{U^{p}} \Lambda_{U^{p}}^{\dagger}\right) \\
& \left.-4 \operatorname{Tr}\left(\Lambda_{U^{l}}^{\dagger} \Lambda_{U^{i}} \tilde{D}\right)\right\}+\left(\Lambda_{U^{i}}\right)_{j k}\left[-\frac{8}{3} g_{3}^{4}+\frac{28}{5} g_{1}^{4}+\frac{64}{15} g_{3}^{2} g_{1}^{2}\right]
\end{aligned}
$$

The renormalisation equations for the bilinear terms can be written as:

$$
\begin{gathered}
\frac{d}{d t} \mu=\beta_{\mu}=\mu\left\{\gamma_{H_{1}}^{H_{1}}+\gamma_{H_{2}}^{H_{2}}\right\}+\kappa^{i} \gamma_{L_{i}}^{H_{1}} \\
\frac{d}{d t} \kappa^{i}=\beta_{k^{i}}=\kappa^{i} \gamma_{H_{2}}^{H_{2}}+\kappa^{p} \gamma_{L_{p}}^{L_{i}}+\mu \gamma_{H_{1}}^{L_{i}}
\end{gathered}
$$

The two loop bilinear $\beta$-functions are given by:

$$
\begin{aligned}
\left(16 \pi^{2}\right)^{2} \beta_{\mu}^{(2)}= & \left(16 \pi^{2}\right)^{2} \beta_{\mu_{(R P C)}}^{(2)}+\mu\left[-\operatorname{Tr}\left(3 E \Lambda_{D^{q}} \Lambda_{D^{q}}^{\dagger}+E \Lambda_{E^{q}} \Lambda_{E^{q}}^{\dagger}+6 \tilde{D} \Lambda_{U^{q}}^{\dagger} \Lambda_{U^{q}}+3 D \Lambda_{D^{q}}^{t} \Lambda_{D^{q}}^{*}\right)\right. \\
& -(\tilde{E})_{l m} \operatorname{Tr}\left(\Lambda_{E^{m}}^{\dagger} \Lambda_{E^{l}}\right)-6(\tilde{D})_{m p} \operatorname{Tr}\left(\Lambda_{D^{p}}^{\dagger} \Lambda_{D^{m}}\right) \\
& \left.-\operatorname{Tr}\left(3 T \Lambda_{D^{q}}^{t} \Lambda_{D^{q}}^{*}\right)-3 \tilde{T}_{l_{p}} \operatorname{Tr}\left(\Lambda_{U^{p}}^{\dagger} \Lambda_{U^{l}}\right)\right] \\
& +\kappa^{i}\left[\left(\frac{2}{5} g_{1}^{2}-16 g_{3}^{2}\right)\left(\Lambda_{D^{q}}^{*} Y_{D}\right)_{i q}-\frac{6}{5} g_{1}^{2}\left(\Lambda_{E^{q}}^{*} Y_{E}\right)_{i q}-\left(3 \Lambda_{E^{l}}^{\dagger} \Lambda_{D^{p}} \Lambda_{D^{p}}^{\dagger} Y_{E}\right)_{i l}\right. \\
& -\left(\Lambda_{E^{l}}^{\dagger} \Lambda_{E^{p}} \Lambda_{E^{p}}^{\dagger} Y_{E}+3 \Lambda_{E^{l}}^{\dagger} E Y_{E}\right)_{i l}-\left(\Lambda_{E^{p}}^{\dagger} Y_{E}\right)_{i l} \operatorname{Tr}\left(\Lambda_{E^{l}}^{\dagger} \Lambda_{E^{p}}\right) \\
& -3\left(E^{t} \Lambda_{D^{m}}^{*} Y_{D}-E^{t} \Lambda_{E^{m}}^{*} Y_{E}\right)_{i m}+9\left(\Lambda_{D^{p}}^{*} D Y_{D}\right)_{i p} \\
& -6\left(\Lambda_{D^{p}}^{*} Y_{D} \Lambda_{U^{m}}^{\dagger} \Lambda_{U^{m}}\right)_{i p}+6\left(\Lambda_{D^{p}}^{*} Y_{D}\right)_{i l} \operatorname{Tr}\left(\Lambda_{D^{l}} \Lambda_{D^{p}}^{\dagger}\right)+3\left(\Lambda_{D^{l}}^{*} T Y_{D}\right)_{i l} \\
& \left.+3\left(\Lambda_{D^{l}}^{*} \Lambda_{D^{m}}^{t} \Lambda_{D^{m}}^{*} Y_{D}\right)_{i l}\right] \\
\left(16 \pi^{2}\right)^{2} \beta_{k^{i}}^{(2)}= & \kappa^{i}\left\{\frac{15}{2} g_{2}^{4}+\frac{207}{50} g_{1}^{4}+\frac{9}{5} g_{2}^{2} g_{1}^{2}\right)+\left(16 g_{3}^{2}+\frac{4}{5} g_{1}^{2}\right) \operatorname{Tr}(T) \\
& \left.-\operatorname{Tr}(9 T T+3 T D)-3 T r\left(T \Lambda_{D^{q}}^{t} \Lambda_{D^{q}}^{*}\right)-3 \tilde{T}_{l_{p}} \operatorname{Tr}\left(\Lambda_{U^{p}}^{\dagger} \Lambda_{U^{l}}\right)\right\} \\
& +\kappa^{p}\left\{\left(16 g_{3}^{2}-\frac{2}{5} g_{1}^{2}\right)\left(\Lambda_{D^{q}} \Lambda_{D^{q}}^{\dagger}\right)_{i p}+\frac{6}{5} g_{1}^{2}\left(E+\Lambda_{E q} \Lambda_{E^{q}}^{\dagger}\right)_{i p}-2\left(E^{2}\right)_{i p}\right.
\end{aligned}
$$




$$
\begin{aligned}
& -\left(Y_{E}^{\dagger}\right)_{k p}\left(Y_{E}\right)_{i l} \operatorname{Tr}\left(\Lambda_{E^{l}}^{\dagger} \Lambda_{E^{k}}\right)-2\left(\Lambda_{E^{l}} \Lambda_{E^{k}}^{\dagger}\right)_{i p}(\tilde{E})_{l k}-E_{i p} \operatorname{Tr}(E+3 D) \\
& -\left(\Lambda_{E^{k}}^{\dagger} E \Lambda_{E^{k}}\right)_{p i}-\left(\Lambda_{E^{l}} \Lambda_{E^{k}}^{\dagger}\right)_{i p} \operatorname{Tr}\left(\Lambda_{E^{l}}^{\dagger} \Lambda_{E^{k}}\right)-\left(Y_{E}\right)_{i k}\left(3 \Lambda_{E^{k}}^{\dagger} \Lambda_{D^{q}} Y_{D}^{*}\right. \\
& \left.+\Lambda_{E^{k}}^{\dagger} \Lambda_{E^{q}} Y_{E}^{*}\right)_{p q}+\left(Y_{E}^{\dagger}\right)_{k p}\left(3 \Lambda_{E^{k}} \Lambda_{D^{q}}^{*} Y_{D}+\Lambda_{E^{k}} \Lambda_{E^{q}}^{*} Y_{E}\right)_{i q} \\
& -\left(3 \Lambda_{E^{k}}^{\dagger} \Lambda_{D^{q}} \Lambda_{D^{q}}^{\dagger} \Lambda_{E^{k}}+\Lambda_{E^{k}}^{\dagger} \Lambda_{E^{q}} \Lambda_{E^{q}}^{\dagger} \Lambda_{E^{k}}\right)_{p^{i}}-6\left(\Lambda_{D^{l}} \Lambda_{D^{k}}^{\dagger}\right)_{i p}\left[(\tilde{D})_{l k}\right. \\
& \left.+\operatorname{Tr}\left(\Lambda_{D^{l}}^{\dagger} \Lambda_{D^{k}}\right)+\left(\Lambda_{U^{q}} \Lambda_{U^{q}}^{\dagger}\right)_{k l}\right]-3\left(\Lambda_{D^{k}}^{*} D \Lambda_{D^{k}}^{t}+\Lambda_{D^{k}}^{*} T \Lambda_{D^{k}}^{t}\right)_{p^{i}} \\
& \left.-3\left(\Lambda_{D^{k}} \Lambda_{D^{q}}^{\dagger} \Lambda_{D^{q}} \Lambda_{D^{k}}^{\dagger}\right)_{i p}\right\}+\mu\left\{\left(\frac{2}{5} g_{1}^{2}-16 g_{3}^{2}\right)\left(\Lambda_{D^{q}} Y_{D}^{*}\right)_{i q}-\frac{6}{5} g_{1}^{2}\left(\Lambda_{E^{q}} Y_{E}^{*}\right)_{i q}\right. \\
& -\left(3 \Lambda_{E^{n}}^{t} \Lambda_{D^{p}}^{*} \Lambda_{D^{p}}^{t} Y_{E}^{*}+\Lambda_{E^{n}}^{t} \Lambda_{E^{p}}^{*} \Lambda_{E^{p}}^{t} Y_{E}^{*}+3 \Lambda_{E^{n}}^{t} E^{t} Y_{E}^{*}\right)_{i n} \\
& -3\left(E \Lambda_{D^{m}} Y_{D}^{*}-E \Lambda_{E^{m}} Y_{E}^{*}\right)_{i m}+3\left(\Lambda_{D^{n}} \Lambda_{D^{m}}^{\dagger} \Lambda_{D^{m}} Y_{D}^{*}\right)_{i n} \\
& -6\left(\Lambda_{D^{q}} Y_{D}^{*} \Lambda_{U^{m}}^{t} \Lambda_{U^{m}}^{*}\right)_{i q}-\left(\Lambda_{E^{q}}^{t} Y_{E}^{*}\right)_{i l} \operatorname{Tr}\left(\Lambda_{E^{l}}^{t} \Lambda_{E^{q}}^{*}\right) \\
& \left.+6\left(\Lambda_{D^{q}} Y_{D}^{*}\right)_{i l} \operatorname{Tr}\left(\Lambda_{D^{l}}^{*} \Lambda_{D^{q}}^{t}\right)+3\left(\Lambda_{D^{n}} Y_{U}^{*} Y_{U}^{t} Y_{D}^{*}\right)_{i n}+9\left(\Lambda_{D^{q}} D^{t} Y_{D}^{*}\right)_{i q}\right\} .
\end{aligned}
$$

\subsection{Soft Supersymmetry Breaking equations}

A precise study of the renormalization group equations must include R-parity violating terms because they have direct and indirect effects on the mass-matrix, and on other couplings of the MSSM lagrangian. R-parity violating couplings allow the generation of lepton-Higgs mixing that results in sneutrino vevs and neutrino masses. By using the method that has been introduced in equations, the RGEs for the bilinear and trilinear soft SUSY breaking terms in R-parity violating scenario can be obtained. They are given by:

$$
\begin{aligned}
& \left(16 \pi^{2}\right)^{2} \beta_{B}^{(2)}=\left(16 \pi^{2}\right)^{2} \beta_{B_{(R P C)}}^{(2)}+B\left\{-T r\left(3 E \Lambda_{D^{q}} \Lambda_{D^{q}}^{\dagger}+E \Lambda_{E^{q}} \Lambda_{E^{q}}^{\dagger}+6 \tilde{D} \Lambda_{U^{q}}^{\dagger} \Lambda_{U^{q}}\right.\right. \\
& \left.+3 D \Lambda_{D^{q}}^{t} \Lambda_{D^{q}}^{*}\right)-\tilde{E}_{l m} \operatorname{Tr}\left(\Lambda_{E^{m}}^{\dagger} \Lambda_{E^{l}}\right)-6(\tilde{D})_{m p} \operatorname{Tr}\left(\Lambda_{D^{p}}^{\dagger} \Lambda_{D^{m}}\right) \\
& \left.-3 \operatorname{Tr}\left(T \Lambda_{D^{q}}^{t} \Lambda_{D^{q}}^{*}\right)-3(\tilde{T})_{l p} \operatorname{Tr}\left(\Lambda_{U^{p}}^{\dagger} \Lambda_{U^{l}}\right)\right\} \\
& +D^{i}\left\{\left(\frac{2}{5} g_{1}^{2}-16 g_{3}^{2}\right)\left(\Lambda_{D^{q}}^{*} Y_{D}\right)_{i q}-\frac{6}{5} g_{1}^{2}\left(\Lambda_{E^{q}}^{*} Y_{E}\right)_{i q}-\left(3 \Lambda_{E^{l}}^{\dagger} \Lambda_{D^{p}} \Lambda_{D^{p}}^{\dagger} Y_{E}\right.\right. \\
& \left.+\Lambda_{E^{l}}^{\dagger} \Lambda_{E^{p}} \Lambda_{E^{p}}^{\dagger} Y_{E}+3 \Lambda_{E^{l}}^{\dagger} E Y_{E}\right)_{i l}-\left(\Lambda_{E^{p}}^{\dagger} Y_{E}\right)_{i l} \operatorname{Tr}\left(\Lambda_{E^{l}}^{\dagger} \Lambda_{E^{p}}\right) \\
& -3\left(E^{t} \Lambda_{D^{m}}^{*} Y_{D}-E^{t} \Lambda_{E^{m}}^{*} Y_{E}\right)_{i m}+9\left(\Lambda_{D^{p}}^{*} D Y_{D}\right)_{i p} \\
& -6\left(\Lambda_{D^{p}}^{*} Y_{D} \Lambda_{U^{m}}^{\dagger} \Lambda_{U^{m}}\right)_{i p}+6\left(\Lambda_{D^{p}}^{*} Y_{D}\right)_{i l} \operatorname{Tr}\left(\Lambda_{D^{l}} \Lambda_{D^{p}}^{\dagger}\right)+3\left(\Lambda_{D^{l}}^{*} T Y_{D}\right)_{i l} \\
& \left.+3\left(\Lambda_{D^{l}}^{*} \Lambda_{D^{m}}^{t} \Lambda_{D^{m}}^{*} Y_{D}\right)_{i l}\right\}-2 \mu\left\{\operatorname{Tr}\left(3 h_{E} Y_{E}^{\dagger} \Lambda_{D^{q}} \Lambda_{D^{q}}^{\dagger}+h_{E} Y_{E^{\prime}}^{\dagger} \Lambda_{E^{q}} \Lambda_{E^{q}}^{\dagger}\right)\right. \\
& +\operatorname{Tr}\left(6 Y_{D}^{\dagger} h_{D} \Lambda_{U^{q}}^{\dagger} \Lambda_{U^{q}}+3 h_{D} Y_{D}^{\dagger} \Lambda_{D^{q}}^{t} \Lambda_{D^{q}}^{*}\right)+6\left(Y_{D}^{\dagger} h_{D}\right)_{n p} \operatorname{Tr}\left(\Lambda_{D^{p}}^{\dagger} \Lambda_{D^{n}}\right) \\
& +\operatorname{Tr}\left(E h_{E^{q}} \Lambda_{E^{q}}^{\dagger}\right)+(\tilde{E})_{l n} \operatorname{Tr}\left(\Lambda_{E^{n}}^{\dagger} h_{E^{l}}\right)+\operatorname{Tr}\left(6 \tilde{D} \Lambda_{U^{q}}^{\dagger} h_{U^{q}}\right) \\
& +\operatorname{Tr}\left(3 E h_{D^{q}} \Lambda_{D^{q}}^{\dagger}+3 D h_{D^{q}}^{t} \Lambda_{D^{q}}^{*}\right)+6(\tilde{D})_{n p} \operatorname{Tr}\left(\Lambda_{D^{p}}^{\dagger} h_{D^{n}}\right) \\
& +\operatorname{Tr}\left(3 h_{U} Y_{U^{\dagger}}^{\dagger} \Lambda_{D^{q}}^{t} \Lambda_{D^{q}}^{*}\right)+\left(Y_{E}^{\dagger} h_{E}\right)_{l n} \operatorname{Tr}\left(\Lambda_{E^{n}}^{\dagger} \Lambda_{E^{l}}\right)+3\left(Y_{U}^{\dagger} h_{U}\right)_{l p} \operatorname{Tr}\left(\Lambda_{U^{p}}^{\dagger} \Lambda_{U^{l}}\right) \\
& \left.+3(\tilde{T})_{l p} \operatorname{Tr}\left(\Lambda_{U^{p}}^{\dagger} h_{U^{l}}\right)+\operatorname{Tr}\left(3 T h_{D^{q}}^{t} \Lambda_{D^{q}}^{*}\right)\right\} \\
& -2 \kappa^{i}\left\{\left(\frac{2}{5} M_{1} g_{1}^{2}-16 M_{3} g_{3}^{2}\right)\left(\Lambda_{D^{q}}^{*} Y_{D}\right)_{i q}-6\left(\Lambda_{D^{q}}^{*} Y_{D}\right)_{i l} \operatorname{Tr}\left(h_{D^{l}} \Lambda_{D^{q}}^{\dagger}\right)\right. \\
& -\frac{6}{5} M_{1} g_{1}^{2}\left(\Lambda_{E^{q}}^{*} Y_{E}\right)_{i q}+\left(16 g_{3}^{2}-\frac{2}{5} g_{1}^{2}\right)\left(\Lambda_{D^{q}}^{*} h_{D}\right)_{i q}+\frac{6}{5} g_{1}^{2}\left(\Lambda_{E^{q}}^{*} h_{E}\right)_{i q} \\
& +\left(3 \Lambda_{E^{l}}^{\dagger} \Lambda_{D^{p}} \Lambda_{D^{p}}^{\dagger} h_{E}+\Lambda_{E^{l}}^{\dagger} \Lambda_{E^{p}} \Lambda_{E^{p}}^{\dagger} h_{E}+3 \Lambda_{E^{l}}^{\dagger} h_{E} \tilde{E}+3 \Lambda_{E^{l}}^{\dagger} E h_{E}\right)_{i l}
\end{aligned}
$$




$$
\begin{aligned}
& +\left(\Lambda_{E^{q}}^{\dagger} h_{E}\right)_{i l} \operatorname{Tr}\left(\Lambda_{E^{l}}^{\dagger} \Lambda_{E^{q}}\right)+3\left(Y_{E}^{*} h_{E}^{t} \Lambda_{D^{m}}^{*} Y_{D}-Y_{E}^{*} h_{E}^{t} \Lambda_{E^{m}}^{*} Y_{E}\right. \\
& \left.-E^{t} \Lambda_{E^{m}}^{*} h_{E}\right)_{i m}-3\left(\Lambda_{D^{l}}^{*} h_{U} Y_{U}^{\dagger} Y_{D}\right)_{i l}-3\left(\Lambda_{D^{l}}^{*} h_{D^{m}}^{t} \Lambda_{D^{m}}^{*} Y_{D}\right)_{i l} \\
& -9\left(\Lambda_{D^{q}}^{*} h_{D} \tilde{D}+\Lambda_{D^{q}}^{*} D h_{D}\right)_{i q}+\left(\Lambda_{E^{q}}^{\dagger} Y_{E}\right)_{i l} \operatorname{Tr}\left(\Lambda_{E^{l}}^{\dagger} h_{E^{q}}\right) \\
& +6\left(\Lambda_{D^{q}}^{*} h_{D} \Lambda_{U^{m}}^{\dagger} \Lambda_{U^{m}}\right)_{i q}-6\left(\Lambda_{D^{q}}^{*} h_{D}\right)_{i l} \operatorname{Tr}\left(\Lambda_{D^{l}} \Lambda_{D^{q}}^{\dagger}\right) \\
& -3\left(\Lambda_{D^{l}}^{*} \Lambda_{D^{m}}^{t} \Lambda_{D^{m}}^{*} h_{D}\right)_{i l}+\left(\Lambda_{E^{l}}^{\dagger} h_{E^{p}} \Lambda_{E^{p}}^{\dagger} Y_{E}\right)_{i l}+3\left(E^{t} \Lambda_{D^{m}}^{*} h_{D}\right)_{i m} \\
& \left.+6\left(\Lambda_{D^{q}}^{*} Y_{D} \Lambda_{U^{m}}^{\dagger} h_{U^{m}}\right)_{i q}+3\left(\Lambda_{E^{l}}^{\dagger} h_{D^{p}} \Lambda_{D^{p}}^{\dagger} Y_{E}\right)_{i l}-3\left(\Lambda_{D^{l}}^{*} T h_{D}\right)_{i l}\right\}
\end{aligned}
$$

$$
\begin{aligned}
& \left(16 \pi^{2}\right)^{2} \beta_{D^{i}}^{(2)}=B\left\{\left(\frac{2}{5} g_{1}^{2}-16 g_{3}^{2}\right)\left(\Lambda_{D^{q}} Y_{D}^{*}\right)_{i q}-\frac{6}{5} g_{1}^{2}\left(\Lambda_{E^{q}} Y_{E}^{*}\right)_{i q}-\left(3 \Lambda_{E^{n}}^{t} \Lambda_{D^{p}}^{*} \Lambda_{D^{p}}^{t} Y_{E}^{*}\right.\right. \\
& \left.+\Lambda_{E^{n}}^{t} \Lambda_{E^{p}}^{*} \Lambda_{E^{p}}^{t} Y_{E}^{*}+3 \Lambda_{E^{n}}^{t} E^{t} Y_{E}^{*}\right)_{i n}-\left(\Lambda_{E^{q}}^{t} Y_{E}^{*}\right)_{i l} \operatorname{Tr}\left(\Lambda_{E^{l}}^{t} \Lambda_{E^{q}}^{*}\right) \\
& -3\left(E \Lambda_{D^{m}} Y_{D}^{*}-E \Lambda_{E^{m}} Y_{E}^{*}\right)_{i m}+9\left(\Lambda_{D^{q}} D^{t} Y_{D}^{*}\right)_{i q} \\
& -6\left(\Lambda_{D^{q}} Y_{D}^{*} \Lambda_{U^{m}}^{t} \Lambda_{U^{m}}^{*}\right)_{i q}+6\left(\Lambda_{D^{q}} Y_{D}^{*}\right)_{i l} \operatorname{Tr}\left(\Lambda_{D^{l}}^{*} \Lambda_{D^{q}}^{t}\right)+3\left(Y_{D}^{\dagger} T \Lambda_{D^{m}}^{t}\right)_{n i} \\
& \left.+3\left(\Lambda_{D^{n}} \Lambda_{D^{m}}^{\dagger} \Lambda_{D^{m}} Y_{D}^{*}\right)_{i n}\right\}+D^{l}\left\{\left(16 g_{3}^{2}-\frac{2}{5} g_{1}^{2}\right)\left(\Lambda_{D^{q}} \Lambda_{D^{q}}^{\dagger}\right)_{i l}\right. \\
& +\frac{6}{5} g_{1}^{2}\left(E+\Lambda_{E^{q}} \Lambda_{E^{q}}^{\dagger}\right)_{i l}+\left(Y_{E}^{\dagger}\right)_{q l}\left(3 \Lambda_{E^{q}} \Lambda_{D^{p}}^{*} Y_{D}+\Lambda_{E^{q}} \Lambda_{E^{p}}^{*} Y_{E}\right)_{i p} \\
& -\left(Y_{E}^{\dagger}\right)_{q l}\left(Y_{E}\right)_{i n} \operatorname{Tr}\left(\Lambda_{E^{n}}^{\dagger} \Lambda_{E^{q}}\right)-2\left(\Lambda_{E^{n}} \Lambda_{E^{q}}^{\dagger}\right)_{i l}(\tilde{E})_{n q} \\
& -\left(\Lambda_{E^{n}} \Lambda_{E^{q}}^{\dagger}\right)_{i l} \operatorname{Tr}\left(\Lambda_{E^{n}}^{\dagger} \Lambda_{E^{q}}\right)-(E)_{i l} \operatorname{Tr}(E+3 D) \\
& -\left(Y_{E}\right)_{i q}\left(3 \Lambda_{E q}^{\dagger} \Lambda_{D^{p}} Y_{D}^{*}+\Lambda_{E^{q}}^{\dagger} \Lambda_{E^{p}} Y_{E}^{*}\right)_{l p}-2\left(E^{2}\right)_{i l} \\
& -\left(\Lambda_{E^{q}}^{\dagger} E \Lambda_{E^{q}}+3 \Lambda_{E^{q}}^{\dagger} \Lambda_{D^{p}} \Lambda_{D^{p}}^{\dagger} \Lambda_{E^{q}}+\Lambda_{E^{q}}^{\dagger} \Lambda_{E^{p}} \Lambda_{E^{p}}^{\dagger} \Lambda_{E^{q}}\right)_{l i} \\
& -6\left(\Lambda_{D^{n}} \Lambda_{D^{q}}^{\dagger}\right)_{i l}\left[(\tilde{D})_{n q}+\operatorname{Tr}\left(\Lambda_{D^{n}}^{\dagger} \Lambda_{D^{q}}\right)+\left(\Lambda_{U^{m}} \Lambda_{U^{m}}^{\dagger}\right)_{q n}\right] \\
& \left.-3\left(\Lambda_{D^{q}}^{*} D \Lambda_{D^{q}}^{t}+\Lambda_{D^{q}}^{*} T \Lambda_{D^{q}}^{t}\right)_{l i}-3\left(\Lambda_{D^{q}} \Lambda_{D^{p}}^{\dagger} \Lambda_{D^{p}} \Lambda_{D^{q}}^{\dagger}\right)_{i l}\right\} \\
& +D^{i}\left\{\left(\frac{15}{2} g_{2}^{4}+\frac{207}{50} g_{1}^{4}+\frac{9}{5} g_{2}^{2} g_{1}^{2}\right)+\left(16 g_{3}^{2}+\frac{4}{5} g_{1}^{2}\right) \operatorname{Tr}(T)\right. \\
& -\operatorname{Tr}\left(9 T^{2}+3 T D+3 T \Lambda_{D^{q}}^{t} \Lambda_{D^{q}}^{*}\right) \\
& \left.-3(\tilde{T})_{l q} \operatorname{Tr}\left(\Lambda_{U^{q}}^{\dagger} \Lambda_{U^{l}}\right)\right\}-2 \kappa^{i}\left\{15 M_{2} g_{2}^{4}+\frac{207}{25} M_{1} g_{1}^{4}\right. \\
& +\frac{9}{5}\left(M_{1}+M_{2}\right) g_{1}^{2} g_{2}^{2}+\left(16 M_{3} g_{3}^{2}+\frac{4}{5} M_{1} g_{1}^{2}\right) \operatorname{Tr}(T) \\
& -\left(16 g_{3}^{2}+\frac{4}{5} g_{1}^{2}\right) \operatorname{Tr}\left(h_{U} Y_{U}^{\dagger}\right)+\operatorname{Tr}\left(9 h_{U} Y_{U}^{\dagger} T+3 h_{U} Y_{U}^{\dagger} D\right. \\
& \left.+3 h_{U} Y_{U}^{\dagger} \Lambda_{D^{q}}^{t} \Lambda_{D^{q}}^{*}+9 T h_{U} Y_{U}^{\dagger}\right)+3\left(Y_{U}^{\dagger} h_{U}\right)_{l q} \operatorname{Tr}\left(\Lambda_{U^{q}}^{\dagger} \Lambda_{U^{l}}\right) \\
& \left.+\operatorname{Tr}\left(3 T h_{D} Y_{D}^{\dagger}\right)+3(\tilde{T})_{l q} \operatorname{Tr}\left(\Lambda_{U^{q}}^{\dagger} h_{U^{l}}\right)+\operatorname{Tr}\left(3 T h_{D^{q}}^{t} \Lambda_{D^{q}}^{*}\right)\right\} \\
& -2 \mu\left\{\left(\frac{2}{5} M_{1} g_{1}^{2}-16 M_{3} g_{3}^{2}\right)\left(\Lambda_{D^{q}} Y_{D}^{*}\right)_{i q}-\frac{6}{5} g_{1}^{2}\left(M_{1} \Lambda_{E^{q}} Y_{E}^{*}-h_{E^{q}} Y_{E}^{*}\right)_{i q}\right. \\
& +\left(16 g_{3}^{2}-\frac{2}{5} g_{1}^{2}\right)\left(h_{D^{q}} Y_{D}^{*}\right)_{i q}+\left(3 \Lambda_{E}^{t} Y_{E}^{*} h_{E}^{t} Y_{E}^{*}\right)_{i n}-3\left(\Lambda_{D^{n}} Y_{U}^{*} h_{U}^{t} Y_{D}^{*}\right)_{i n} \\
& +3\left(h_{E} Y_{E}^{\dagger} \Lambda_{D^{m}} Y_{D}^{*}-h_{E} Y_{E}^{\dagger} \Lambda_{E^{m}} Y_{E}^{*}\right)_{i m}-9\left(\Lambda_{D^{q}} Y_{D}^{*} h_{D}^{t} Y_{D}^{*}\right)_{i q} \\
& +\left(3 h_{E^{n}}^{t} \Lambda_{D^{p}}^{*} \Lambda_{D^{p}}^{t} Y_{E}^{*}+h_{E^{n}}^{t} \Lambda_{E^{p}}^{*} \Lambda_{E^{p}}^{t} Y_{E}^{*}+\Lambda_{E^{n}}^{t} \Lambda_{E^{p}}^{*} h_{E^{p}}^{t} Y_{E}^{*}\right. \\
& \left.+3 h_{E^{n}}^{t} E^{t} Y_{E}^{*}\right)_{i n}+\left(h_{E^{q}}^{t} Y_{E}^{*}\right)_{i l} \operatorname{Tr}\left(\Lambda_{E^{l}}^{t} \Lambda_{E^{q}}^{*}\right)+\left(\Lambda_{E^{q}}^{t} Y_{E}^{*}\right)_{i l} \operatorname{Tr}\left(h_{E^{l}}^{t} \Lambda_{E^{q}}^{*}\right) \\
& -3\left(E h_{E^{m}} Y_{E}^{*}\right)_{i m}+6\left(\Lambda_{D^{q}} Y_{D}^{*} h_{U^{m}}^{t} \Lambda_{U^{m}}^{*}\right)_{i q}+3\left(\Lambda_{E^{n}}^{t} \Lambda_{D^{p}}^{*} h_{D^{p}}^{t} Y_{E}^{*}\right)_{i n} \\
& +3\left(E h_{D^{m}} Y_{D}^{*}\right)_{i m}-9\left(h_{D^{q}} D^{t} Y_{D}^{*}\right)_{i q}+6\left(h_{D^{q}} Y_{D}^{*} \Lambda_{U^{m}}^{t} \Lambda_{U^{m}}^{*}\right)_{i q}
\end{aligned}
$$




$$
\begin{aligned}
& -6\left(h_{D^{q}} Y_{D}^{*}\right)_{i l} \operatorname{Tr}\left(\Lambda_{D^{l}}^{*} \Lambda_{D^{q}}^{t}\right)-6\left(\Lambda_{D^{q}} Y_{D}^{*}\right)_{i l} \operatorname{Tr}\left(\Lambda_{D^{l}}^{*} h_{D^{q}}^{t}\right) \\
& \left.-3\left(Y_{D}^{\dagger} T h_{D^{n}}^{t}\right)_{n i}-3\left(h_{D^{n}} \Lambda_{D^{m}}^{\dagger} \Lambda_{D^{m}} Y_{D}^{*}+\Lambda_{D^{n}} \Lambda_{D^{m}}^{\dagger} h_{D^{m}} Y_{D}^{*}\right)_{i n}\right\} \\
& -2 \kappa^{l}\left\{\left(16 M_{3} g_{3}^{2}-\frac{2}{5} M_{1} g_{1}^{2}\right)\left(\Lambda_{D^{q}} \Lambda_{D^{q}}^{\dagger}\right)_{i l}+\frac{6}{5} M_{1} g_{1}^{2}\left(E+\Lambda_{E^{q}} \Lambda_{E^{q}}^{\dagger}\right)_{i l}\right. \\
& -\left(16 g_{3}^{2}-\frac{2}{5} g_{1}^{2}\right)\left(h_{D^{q}} \Lambda_{D^{q}}^{\dagger}\right)_{i l}-\frac{6}{5} g_{1}^{2}\left(h_{E} Y_{E}^{\dagger}+h_{E^{q}} \Lambda_{E^{q}}^{\dagger}\right)_{i l} \\
& +2\left(h_{E} Y_{E}^{\dagger} E+E h_{E} Y_{E}^{\dagger}\right)_{i l}+\left(h_{E} Y_{E}^{\dagger}\right)_{i l} \operatorname{Tr}(E+3 D) \\
& +\left(Y_{E}^{\dagger}\right)_{q l}\left(h_{E}\right)_{i n} \operatorname{Tr}\left(\Lambda_{E^{n}}^{\dagger} \Lambda_{E^{q}}\right)+2\left(\Lambda_{E^{n}} \Lambda_{E^{q}}^{\dagger}\right)_{i l}\left(Y_{E}^{\dagger} h_{E}\right)_{n q} \\
& +(E)_{i l} \operatorname{Tr}\left(h_{E} Y_{E}^{\dagger}\right)+\left(h_{E}\right)_{i q}\left(3 \Lambda_{E^{q}}^{\dagger} \Lambda_{D^{p}} Y_{D}^{*}+\Lambda_{E^{q}}^{\dagger} \Lambda_{E^{p}} Y_{E}^{*}\right)_{l p} \\
& -\left(Y_{E}^{\dagger}\right)_{q l}\left(\Lambda_{E^{q}} \Lambda_{E^{p}}^{*} h_{E}\right)_{i p}+\left(\Lambda_{E^{q}}^{\dagger} h_{E} Y_{E}^{\dagger} \Lambda_{E^{q}}\right)_{l i}+3\left(\Lambda_{D^{q}}^{*} h_{U} Y_{U^{\dagger}}^{\dagger} \Lambda_{D^{q}}^{t}\right)_{l i} \\
& +(E)_{i l} \operatorname{Tr}\left(3 h_{D} Y_{D}^{\dagger}\right)-3\left(Y_{E}^{*}\right)_{l q}\left(\Lambda_{E^{q}} \Lambda_{D^{p}}^{*} h_{D}\right)_{i p} \\
& +6\left(\Lambda_{D^{n}} \Lambda_{D^{q}}^{\dagger}\right)_{i l}\left(Y_{D}^{\dagger} h_{D}\right)_{n q}+3\left(\Lambda_{D^{q}}^{*} h_{D} Y_{D}^{\dagger} \Lambda_{D^{q}}^{t}\right)_{l i} \\
& +2\left(h_{E^{n}} \Lambda_{E^{q}}^{\dagger}\right)_{i l}(\tilde{E})_{n q}+\left(h_{E^{n}} \Lambda_{E^{q}}^{\dagger}\right)_{i l} \operatorname{Tr}\left(\Lambda_{E^{n}}^{\dagger} \Lambda_{E^{q}}\right)+\left(\Lambda_{E^{n}} \Lambda_{E^{q}}^{\dagger}\right)_{i l} \operatorname{Tr}\left(\Lambda_{E^{n}}^{\dagger} h_{E^{q}}\right) \\
& +\left(Y_{E}\right)_{i q}\left(\Lambda_{E q}^{\dagger} h_{E^{p}} Y_{E}^{*}\right)_{l p}-\left(Y_{E}^{\dagger}\right)_{q l}\left(3 h_{E^{q}} \Lambda_{D^{p}}^{*} Y_{D}+h_{E^{q}} \Lambda_{E p}^{*} Y_{E}\right)_{i p} \\
& +\left(\Lambda_{E^{q}}^{\dagger} E h_{E^{q}}+3 \Lambda_{E^{q}}^{\dagger} \Lambda_{D^{p}} \Lambda_{D^{p}}^{\dagger} h_{E^{q}}+\Lambda_{E^{q}}^{\dagger} h_{E^{p}} \Lambda_{E^{p}}^{\dagger} \Lambda_{E^{q}}+\Lambda_{E^{q}}^{\dagger} \Lambda_{E^{p}} \Lambda_{E^{p}}^{\dagger} h_{E^{q}}\right)_{l i} \\
& +6\left(\Lambda_{D^{n}} \Lambda_{D^{q}}^{\dagger}\right)_{i l}\left(h_{U^{m}} \Lambda_{U^{m}}^{\dagger}\right)_{q n}+3\left(Y_{E}\right)_{i q}\left(\Lambda_{E^{q}}^{\dagger} h_{D^{p}} Y_{D}^{*}\right)_{l p}+3\left(\Lambda_{E^{q}}^{\dagger} h_{D^{p}} \Lambda_{D^{p}}^{\dagger} \Lambda_{E^{q}}\right)_{l i} \\
& +6\left(h_{D^{n}} \Lambda_{D^{q}}^{\dagger}\right)_{i l}\left[(\tilde{D})_{n q}+\operatorname{Tr}\left(\Lambda_{D^{n}}^{\dagger} \Lambda_{D^{q}}\right)+\left(\Lambda_{U^{m}} \Lambda_{U^{m}}^{\dagger}\right)_{q n}\right] \\
& +6\left(\Lambda_{D^{n}} \Lambda_{D^{q}}^{\dagger}\right)_{i l} \operatorname{Tr}\left(\Lambda_{D^{n}}^{\dagger} h_{D^{q}}\right)+3\left(\Lambda_{D^{q}}^{*} D h_{D^{q}}^{t}+\Lambda_{D^{q}}^{*} T h_{D^{q}}^{t}\right)_{l i} \\
& \left.+3\left(\Lambda_{D^{q}} \Lambda_{D^{p}}^{\dagger} h_{D^{p}} \Lambda_{D^{q}}^{\dagger}+h_{D^{q}} \Lambda_{D^{p}}^{\dagger} \Lambda_{D^{p}} \Lambda_{D^{q}}^{\dagger}\right)_{i l}+\left(Y_{E}^{\dagger}\right)_{q l}\left(Y_{E}\right)_{i n} \operatorname{Tr}\left(\Lambda_{E^{n}}^{\dagger} h_{E^{q}}\right)\right\} \text { (40) }
\end{aligned}
$$

$$
\begin{aligned}
& \left(16 \pi^{2}\right)^{2} \beta_{\left(h_{E}\right)_{i j}}^{(2)}=\left(16 \pi^{2}\right)^{2} \beta_{\left(h_{E}\right)_{(R P C)}}^{(2)}+\left(h_{E}\right)_{l j}\left\{\left(16 g_{3}^{2}-\frac{2}{5} g_{1}^{2}\right)\left(\Lambda_{D^{q}} \Lambda_{D^{q}}^{\dagger}\right)_{i l}+\frac{6}{5} g_{1}^{2}\left(\Lambda_{E^{q}} \Lambda_{E^{q}}^{\dagger}\right)_{i l}\right. \\
& -\left(Y_{E}^{\dagger}\right)_{m l}\left(Y_{E}\right)_{i q} \operatorname{Tr}\left(\Lambda_{E^{q}}^{\dagger} \Lambda_{E^{m}}\right)-2\left(\Lambda_{E^{n}} \Lambda_{E^{m}}^{\dagger}\right)_{i l}(\tilde{E})_{n m} \\
& -\left(\Lambda_{E^{n}} \Lambda_{E^{m}}^{\dagger}\right)_{i l} \operatorname{Tr}\left(\Lambda_{E^{n}}^{\dagger} \Lambda_{E^{m}}\right)+\left(Y_{E}^{*}\right)_{l q}\left(3 \Lambda_{E^{q}} \Lambda_{D^{p}}^{*} Y_{D}+\Lambda_{E^{q}} \Lambda_{E^{p}}^{*} Y_{E}\right)_{i p} \\
& -\left(Y_{E}\right)_{i q}\left(3 \Lambda_{E q}^{\dagger} \Lambda_{D^{p}} Y_{D}^{*}+\Lambda_{E^{q}}^{\dagger} \Lambda_{E^{p}} Y_{E}^{*}\right)_{l p}-\left(\Lambda_{E^{q}}^{\dagger} E \Lambda_{E^{q}}+3 \Lambda_{E q}^{\dagger} \Lambda_{D^{p}} \Lambda_{D^{p}}^{\dagger} \Lambda_{E^{q}}\right. \\
& \left.+\Lambda_{E^{q}}^{\dagger} \Lambda_{E^{p}} \Lambda_{E^{p}}^{\dagger} \Lambda_{E^{q}}\right)_{l i}-6\left(\Lambda_{D^{n}} \Lambda_{D^{m}}^{\dagger}\right)_{i l}\left[\tilde{D}_{n m}+\operatorname{Tr}\left(\Lambda_{D^{n}}^{\dagger} \Lambda_{D^{m}}\right)\right. \\
& \left.\left.+\left(\Lambda_{U^{q}} \Lambda_{U^{q}}^{\dagger}\right)_{m n}\right]-3\left(\Lambda_{D^{q}}^{*} D \Lambda_{D^{q}}^{t}+\Lambda_{D^{q}}^{*} T \Lambda_{D^{q}}^{t}\right)_{l i}-3\left(\Lambda_{D^{q}} \Lambda_{D^{p}}^{\dagger} \Lambda_{D^{p}} \Lambda_{D^{q}}^{\dagger}\right)_{i l}\right\} \\
& -\left(h_{E}\right)_{i j}\left\{\operatorname{Tr}\left(3 E \Lambda_{D^{q}} \Lambda_{D^{q}}^{\dagger}+E \Lambda_{E^{q}} \Lambda_{E^{q}}^{\dagger}+6 \tilde{D} \Lambda_{U^{q}}^{\dagger} \Lambda_{U^{q}}+3 D \Lambda_{D^{q}}^{t} \Lambda_{D^{q}}^{*}\right)\right. \\
& \left.+\tilde{E}_{m l} \operatorname{Tr}\left(\Lambda_{E^{l}}^{\dagger} \Lambda_{E^{m}}\right)+6(\tilde{D})_{l q} \operatorname{Tr}\left(\Lambda_{D^{q}}^{\dagger} \Lambda_{D^{l}}\right)\right\} \\
& +\left(h_{E}\right)_{i l}\left\{\left(3 g_{2}^{2}-\frac{3}{5} g_{1}^{2}\right) \operatorname{Tr}\left(\Lambda_{E^{j}} \Lambda_{E^{l}}^{\dagger}\right)\right. \\
& -2\left(Y_{E}^{\dagger} \Lambda_{E^{n}} \Lambda_{E^{n}}^{\dagger} Y_{E}+3 Y_{E}^{\dagger} \Lambda_{D^{n}} \Lambda_{D^{n}}^{\dagger} Y_{E}\right)_{l j}+6\left(Y_{E}^{\dagger} \Lambda_{E^{j}} \Lambda_{D^{m}}^{*} Y_{D}\right)_{l m} \\
& -2\left(Y_{E}^{\dagger} \Lambda_{E^{j}} \Lambda_{E^{m}}^{\dagger} Y_{E}\right)_{l m}-2 \operatorname{Tr}\left(\Lambda_{E^{j}} \Lambda_{E^{l}}^{\dagger} E\right) \\
& -2 \operatorname{Tr}\left(\Lambda_{E^{j}} \Lambda_{E^{l}}^{\dagger} \Lambda_{E^{n}} \Lambda_{E^{n}}^{\dagger}\right)-6 \operatorname{Tr}\left(\Lambda_{E^{j}} \Lambda_{E^{l}}^{\dagger} \Lambda_{D^{n}} \Lambda_{D^{n}}^{\dagger}\right)+6\left(Y_{D^{\dagger}}^{\dagger} \Lambda_{D^{m}}^{\dagger} \Lambda_{E^{l}}^{\dagger} Y_{E}\right)_{m j} \\
& \left.-2\left(Y_{E}^{\dagger} \Lambda_{E^{m}} \Lambda_{E^{l}}^{\dagger} Y_{E}\right)_{m j}\right\}+\left(h_{E^{j}}\right)_{i l}\left\{\left(\frac{2}{5} g_{1}^{2}-16 g_{3}^{2}\right)\left(\Lambda_{D^{q}}^{*} Y_{D}\right)_{l q}-\frac{6}{5} g_{1}^{2}\left(\Lambda_{E^{q}}^{*} Y_{E}\right)_{l q}\right. \\
& -3\left(\Lambda_{E^{k}}^{\dagger} \Lambda_{D^{p}} \Lambda_{D^{p}}^{\dagger} Y_{E}\right)_{l k}-\left(\Lambda_{E^{k}}^{\dagger} \Lambda_{E^{p}} \Lambda_{E^{p}}^{\dagger} Y_{E}\right)_{l k}-3\left(\Lambda_{E^{k}}^{\dagger} E Y_{E}\right)_{l k} \\
& -\left(\Lambda_{E^{q}}^{\dagger} Y_{E}\right)_{l n} \operatorname{Tr}\left(\Lambda_{E^{n}}^{\dagger} \Lambda_{E^{q}}\right)-3\left(E^{t} \Lambda_{D^{m}}^{*} Y_{D}-E^{t} \Lambda_{E^{m}}^{*} Y_{E}\right)_{l m}+9\left(\Lambda_{D^{q}}^{*} D Y_{D}\right)_{l q} \\
& -6\left(\Lambda_{D^{q}}^{*} Y_{D} \Lambda_{U^{m}}^{\dagger} \Lambda_{U^{m}}\right)_{l q}+6\left(\Lambda_{D^{q}}^{*} Y_{D}\right)_{l n} \operatorname{Tr}\left(\Lambda_{D^{n}} \Lambda_{D^{q}}^{\dagger}\right)+3\left(\Lambda_{D^{k}}^{*} T Y_{D}\right)_{l k} \\
& \left.+3\left(\Lambda_{D^{k}}^{*} \Lambda_{D^{m}}^{t} \Lambda_{D^{m}}^{*} Y_{D}\right)_{l k}\right\}-2\left(Y_{E}\right)_{l j}\left\{\left(16 M_{3} g_{3}^{2}-\frac{2}{5} M_{1} g_{1}^{2}\right)\left(\Lambda_{D^{q}} \Lambda_{D^{q}}^{\dagger}\right)_{i l}\right.
\end{aligned}
$$




$$
\begin{aligned}
& +\frac{6}{5} M_{1} g_{1}^{2}\left(\Lambda_{E^{q}} \Lambda_{E^{q}}^{\dagger}\right)_{i l}-\left(16 g_{2}^{3}-\frac{2}{5} g_{1}\right)\left(h_{D^{q}} \Lambda_{D^{q}}^{\dagger}\right)_{i l}-\frac{6}{5} g_{1}^{2}\left(h_{E^{q}} \Lambda_{E^{q}}^{\dagger}\right)_{i l} \\
& +\left(Y_{E}^{\dagger}\right)_{q l}\left(h_{E}\right)_{i n} \operatorname{Tr}\left(\Lambda_{E^{n}}^{\dagger} \Lambda_{E^{q}}\right)+2\left(\Lambda_{E^{n}} \Lambda_{E^{q}}^{\dagger}\right)_{i l}\left(Y_{E}^{\dagger} h_{E}\right)_{n q} \\
& +\left(h_{E}\right)_{i q}\left(3 \Lambda_{E^{q}}^{\dagger} \Lambda_{D^{p}} Y_{D}^{*}+\Lambda_{E^{q}}^{\dagger} \Lambda_{E^{p}} Y_{E}^{*}\right)_{l p}-\left(Y_{E}^{*}\right)_{l q}\left(\Lambda_{E^{q}} \Lambda_{E^{p}}^{*} h_{E}\right)_{i p} \\
& -3\left(Y_{E}^{*}\right)_{l q}\left(\Lambda_{E^{q}} \Lambda_{D^{p}}^{*} h_{D}\right)_{i p}+6\left(\Lambda_{D^{n}} \Lambda_{D^{q}}^{\dagger}\right)_{i l}\left(Y_{D}^{\dagger} h_{D}\right)_{n q}+3\left(\Lambda_{D^{q}}^{*} h_{D} Y_{D}^{\dagger} \Lambda_{D^{q}}^{t}\right)_{l i} \\
& +\left(\Lambda_{E}^{\dagger} h_{E} Y_{E}^{\dagger} \Lambda_{E^{q}}\right)_{l i}+3\left(\Lambda_{D^{q}}^{*} h_{U} Y_{U}^{\dagger} \Lambda_{D^{q}}^{t}\right)_{l i}+\left(h_{E^{n}} \Lambda_{E^{q}}^{\dagger}\right)_{i l} \operatorname{Tr}\left(\Lambda_{E^{n}}^{\dagger} \Lambda_{E^{q}}\right) \\
& +\left(Y_{E}^{\dagger}\right)_{q l}\left(Y_{E}\right)_{i n} \operatorname{Tr}\left(\Lambda_{E^{n}}^{\dagger} h_{E^{q}}\right)+2\left(h_{E^{n}} \Lambda_{E^{q}}^{\dagger}\right)_{i l}(\tilde{E})_{n q} \\
& +\left(\Lambda_{E^{n}} \Lambda_{E^{q}}^{\dagger}\right)_{i l} \operatorname{Tr}\left(\Lambda_{E^{n}}^{\dagger} h_{E^{q}}\right)+\left(Y_{E}\right)_{i q}\left(\Lambda_{E^{q}}^{\dagger} h_{E^{p}} Y_{E}^{*}\right)_{l p}-\left(Y_{E}^{*}\right)_{l q}\left(3 h_{E^{q}} \Lambda_{D^{p}}^{*} Y_{D}\right. \\
& \left.+h_{E^{q}} \Lambda_{E^{p}}^{*} Y_{E}\right)_{i p}+\left(\Lambda_{E^{q}}^{\dagger} E h_{E^{q}}\right)_{l i}+3\left(\Lambda_{E^{q}}^{\dagger} \Lambda_{D^{p}} \Lambda_{D^{p}}^{\dagger} h_{E^{q}}\right)_{l i} \\
& +\left(\Lambda_{E^{q}}^{\dagger} h_{E^{p}} \Lambda_{E^{p}}^{\dagger} \Lambda_{E^{q}}+\Lambda_{E^{q}}^{\dagger} \Lambda_{E^{p}} \Lambda_{E^{p}}^{\dagger} h_{E^{q}}\right)_{l i}+6\left(\Lambda_{D^{n}} \Lambda_{D^{q}}^{\dagger}\right)_{i l}\left(h_{U^{m}} \Lambda_{U^{m}}^{\dagger}\right)_{q n} \\
& +3\left(Y_{E}\right)_{i q}\left(\Lambda_{E^{q}}^{\dagger} h_{D^{p}} Y_{D}^{*}\right)_{l p}+3\left(\Lambda_{E^{q}}^{\dagger} h_{D^{p}} \Lambda_{D^{p}}^{\dagger} \Lambda_{E^{q}}\right)_{l i}+6\left(h_{D^{n}} \Lambda_{D^{q}}^{\dagger}\right)_{i l}\left[(\tilde{D})_{n q}\right. \\
& \left.+\operatorname{Tr}\left(\Lambda_{D^{n}}^{\dagger} \Lambda_{D^{q}}\right)+\left(\Lambda_{U^{m}} \Lambda_{U^{m}}^{\dagger}\right)_{q n}\right]+6\left(\Lambda_{D^{n}} \Lambda_{D^{q}}^{\dagger}\right)_{i l} \operatorname{Tr}\left(\Lambda_{D^{n}}^{\dagger} h_{D^{q}}\right)+3\left(\Lambda_{D^{q}}^{*} D h_{D^{q}}^{t}\right)_{l i} \\
& \left.+3\left(\Lambda_{D^{q}}^{*} T h_{D^{q}}^{t}\right)_{l i}+3\left(\Lambda_{D^{q}} \Lambda_{D^{p}}^{\dagger} h_{D^{p}} \Lambda_{D^{q}}^{\dagger}+h_{D^{q}} \Lambda_{D^{p}}^{\dagger} \Lambda_{D^{p}} \Lambda_{D^{q}}^{\dagger}\right)_{i l}\right\} \\
& -2\left(Y_{E}\right)_{i j}\left\{\operatorname{Tr}\left(3 h_{E} Y_{E}^{\dagger} \Lambda_{D^{q}} \Lambda_{D^{q}}^{\dagger}+h_{E} Y_{E}^{\dagger} \Lambda_{E q} \Lambda_{E^{q}}^{\dagger}\right)+6(\tilde{D}){ }_{l q} \operatorname{Tr}\left(\Lambda_{D^{q}}^{\dagger} h_{D^{l}}\right)\right. \\
& +6 \operatorname{Tr}\left(Y_{D}^{\dagger} h_{D} \Lambda_{U^{q}}^{\dagger} \Lambda_{U^{q}}\right)+3 \operatorname{Tr}\left(h_{D} Y_{D}^{\dagger} \Lambda_{D^{q}}^{t} \Lambda_{D^{q}}^{*}\right)+\left(Y_{E}^{\dagger} h_{E}\right)_{m n} \operatorname{Tr}\left(\Lambda_{E^{n}}^{\dagger} \Lambda_{E^{m}}\right)
\end{aligned}
$$

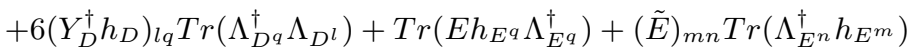

$$
\begin{aligned}
& \left.+6 \operatorname{Tr}\left(\tilde{D} \Lambda_{U^{q}}^{\dagger} h_{U^{q}}\right)+3 \operatorname{Tr}\left(E h_{D^{q}} \Lambda_{D^{q}}^{\dagger}+D h_{D^{q}}^{t} \Lambda_{D^{q}}^{*}\right)\right\} \\
& -2\left(Y_{E}\right)_{i l}\left\{\left(3 M_{2} g_{2}^{2}-\frac{3}{5} M_{1} g_{1}^{2}\right) \operatorname{Tr}\left(\Lambda_{E^{j}} \Lambda_{E^{l}}^{\dagger}\right)+\left(\frac{3}{5} g_{1}^{2}-3 g_{2}^{2}\right) \operatorname{Tr}\left(h_{E^{j}} \Lambda_{E^{l}}^{\dagger}\right)\right. \\
& +6\left(Y_{E}^{\dagger} \Lambda_{D^{q}} \Lambda_{D^{q}}^{\dagger} h_{E}\right)_{l j}+2\left(Y_{E}^{\dagger} \Lambda_{E^{j}} \Lambda_{E^{m}}^{\dagger} h_{E}\right)_{l m}+2 \operatorname{Tr}\left(\Lambda_{E^{j}} \Lambda_{E^{l}}^{\dagger} h_{E} Y_{E}^{\dagger}\right) \\
& -6\left(Y_{D}^{\dagger} \Lambda_{D^{m}}^{t} \Lambda_{E^{l}}^{\dagger} h_{E}\right)_{m j}+2\left(Y_{E}^{\dagger} \Lambda_{E^{m}} \Lambda_{E^{l}}^{\dagger} h_{E}\right)_{m j} \\
& +2\left(Y_{E}^{\dagger} h_{E^{q}} \Lambda_{E^{q}}^{\dagger} Y_{E}\right)_{l j}-6\left(Y_{E}^{\dagger} h_{E^{j}} \Lambda_{D^{m}}^{*} Y_{D}\right)_{l m}+2\left(Y_{E}^{\dagger} h_{E^{j}} \Lambda_{E^{m}}^{\dagger} Y_{E}\right)_{l m} \\
& +2 \operatorname{Tr}\left[h_{E^{j}} \Lambda_{E^{l}}^{\dagger}\left(E+\Lambda_{E^{q}} \Lambda_{E^{q}}^{\dagger}\right)\right]+6 \operatorname{Tr}\left(h_{E^{j}} \Lambda_{E^{l}}^{\dagger} \Lambda_{D^{q}} \Lambda_{D^{q}}^{\dagger}\right)+2 \operatorname{Tr}\left(\Lambda_{E^{j}} \Lambda_{E^{l}}^{\dagger} h_{E^{q}} \Lambda_{E^{q}}^{\dagger}\right) \\
& +2\left(Y_{E}^{\dagger} h_{E^{m}} \Lambda_{E^{l}}^{\dagger} Y_{E}\right)_{m j}+6\left(Y_{E}^{\dagger} h_{D^{q}} \Lambda_{D^{q}}^{\dagger} Y_{E}\right)_{l j}+6 \operatorname{Tr}\left(\Lambda_{E^{j}} \Lambda_{E^{l}}^{\dagger} h_{D^{q}} \Lambda_{D^{q}}^{\dagger}\right) \\
& \left.-6\left(Y_{D}^{\dagger} h_{D^{m}}^{t} \Lambda_{E^{l}}^{\dagger} Y_{E}\right)_{m j}+2\left(Y_{E}^{\dagger} \Lambda_{E^{q}} \Lambda_{E^{q}}^{\dagger} h_{E}\right)_{l j}-6\left(Y_{E}^{\dagger} \Lambda_{E^{j}} \Lambda_{D^{m}}^{*} h_{D}\right)_{l m}\right\} \\
& -2\left(\Lambda_{E^{j}}\right)_{i l}\left\{\left(\frac{2}{5} M_{1} g_{1}^{2}-16 M_{3} g_{3}^{2}\right)\left(\Lambda_{D^{q}}^{*} Y_{D}\right)_{l q}-\frac{6}{5} M_{1} g_{1}^{2}\left(\Lambda_{E^{q}}^{*} Y_{E}\right)_{l q}\right. \\
& +\left(16 g_{3}^{2}-\frac{2}{5} g_{1}^{2}\right)\left(\Lambda_{D^{q}}^{*} h_{D}\right)_{l q}+\frac{6}{5} g_{1}^{2}\left(\Lambda_{E^{q}}^{*} h_{E}\right)_{l q}-6\left(\Lambda_{D^{q}}^{*} h_{D}\right) \ln \operatorname{Tr}\left(\Lambda_{D^{n}} \Lambda_{D^{q}}^{\dagger}\right) \\
& +3\left(\Lambda_{E^{k}}^{\dagger} \Lambda_{D^{p}} \Lambda_{D^{p}}^{\dagger} h_{E}+\Lambda_{E^{k}}^{\dagger} h_{E} Y_{E}^{\dagger} Y_{E}+\Lambda_{E^{k}}^{\dagger} E h_{E}\right)_{l k} \\
& +\left(\Lambda_{E^{k}}^{\dagger} \Lambda_{E^{p}} \Lambda_{E^{p}}^{\dagger} h_{E}\right)_{l k}+\left(\Lambda_{E^{q}}^{\dagger} h_{E}\right)_{l n} \operatorname{Tr}\left(\Lambda_{E^{n}}^{\dagger} \Lambda_{E^{q}}\right)+3\left(Y_{E}^{*} h_{E}^{t} \Lambda_{D^{m}}^{*} Y_{D}\right)_{l m} \\
& -3\left(Y_{E}^{*} h_{E}^{t} \Lambda_{E^{m}}^{*} Y_{E}+E^{t} \Lambda_{E^{m}}^{*} h_{E}\right)_{l m}-3\left(\Lambda_{D^{k}}^{*} h_{U} Y_{U}^{\dagger} Y_{D}\right)_{l k} \\
& -9\left(\Lambda_{D^{q}}^{*} h_{D} \tilde{D}+\Lambda_{D^{q}}^{*} D h_{D}\right)_{l q}+6\left(\Lambda_{D^{q}}^{*} h_{D} \Lambda_{U^{m}}^{\dagger} \Lambda_{U^{m}}\right)_{l q} \\
& -3\left(\Lambda_{D^{k}}^{*} T h_{D}\right)_{l k}-3\left(\Lambda_{D^{k}}^{*} \Lambda_{D^{m}}^{t} \Lambda_{D^{m}}^{*} h_{D}\right)_{l k}+\left(\Lambda_{E^{k}}^{\dagger} h_{E^{p}} \Lambda_{E^{p}}^{\dagger} Y_{E}\right)_{l k} \\
& +\left(\Lambda_{E q}^{\dagger} Y_{E}\right)_{l n} \operatorname{Tr}\left(\Lambda_{E^{n}}^{\dagger} h_{E^{q}}\right)+6\left(\Lambda_{D^{q}}^{*} Y_{D} \Lambda_{U^{m}}^{\dagger} h_{U^{m}}\right)_{l q}+\left(\Lambda_{E^{k}}^{\dagger} h_{D^{p}} \Lambda_{D^{p}}^{\dagger} Y_{E}\right)_{l k} \\
& \left.-6\left(\Lambda_{D^{q}}^{*} Y_{D}\right)_{l n} \operatorname{Tr}\left(h_{D^{n}} \Lambda_{D^{q}}^{\dagger}\right)-3\left(\Lambda_{D^{k}}^{*} h_{D^{m}}^{t} \Lambda_{D^{m}}^{*} Y_{D}\right)_{l k}+3\left(E^{t} \Lambda_{D^{m}}^{*} h_{D}\right)_{l m}\right\} \\
& \left(16 \pi^{2}\right)^{2} \beta_{\left(h_{D}\right)_{i j}}^{(2)}=\left(16 \pi^{2}\right)^{2} \beta_{\left(h_{D}\right)_{(R P C)}^{(2)}}^{(2)}+\left(h_{D}\right)_{l j}\left\{\frac{2}{5} g_{1}^{2}\left(\Lambda_{D^{q}}^{\dagger} \Lambda_{D^{q}}\right)_{l i}-2\left(\Lambda_{D^{n}}^{\dagger} \Lambda_{D^{m}}\right)_{l i}\left[(\tilde{D})_{m n}+\left(\Lambda_{U^{q}} \Lambda_{U^{q}}^{\dagger}\right)_{n m}\right.\right. \\
& \left.+\operatorname{Tr}\left(\Lambda_{D^{m}}^{\dagger} \Lambda_{D^{n}}\right)\right]-\left(\Lambda_{D^{m}}^{\dagger} E \Lambda_{D^{m}}\right)_{l i}-3\left(\Lambda_{D^{m}}^{\dagger} \Lambda_{D^{q}} \Lambda_{D^{q}}^{\dagger} \Lambda_{D^{m}}\right)_{l i} \\
& -\left(\Lambda_{D^{m}}^{\dagger} \Lambda_{E^{q}} \Lambda_{E^{q}}^{\dagger} \Lambda_{D^{m}}\right)_{l i}-3\left(Y_{D}\right)_{i n}\left(\Lambda_{D^{n}}^{\dagger} \Lambda_{D^{m}} Y_{D}^{*}\right)_{l m}-\left(Y_{D}\right)_{i n}\left(\Lambda_{D^{n}}^{\dagger} \Lambda_{E^{m}} Y_{E}^{*}\right)_{l m}
\end{aligned}
$$




$$
\begin{aligned}
& -2\left(Y_{D} \Lambda_{U^{q}}^{\dagger} \Lambda_{U^{q}} Y_{D}^{\dagger}\right)_{i l}-2\left(Y_{D}\right)_{i m}\left(Y_{D}^{\dagger}\right)_{n l} \operatorname{Tr}\left(\Lambda_{D^{m}}^{\dagger} \Lambda_{D^{n}}\right) \\
& \left.-\left(Y_{D}^{*}\right)_{l n}\left(3 \Lambda_{D^{n}}^{t} \Lambda_{D^{m}}^{*} Y_{D}+\Lambda_{D^{n}}^{t} \Lambda_{E^{m}}^{*} Y_{E}\right)_{i m}-\left(Y_{U}\right)_{i n}\left(Y_{U}^{\dagger}\right)_{q l} \operatorname{Tr}\left(\Lambda_{U^{n}}^{\dagger} \Lambda_{U^{q}}\right)\right\} \\
& -\left(h_{D}\right)_{i j}\left\{3 \operatorname{Tr}\left(D \Lambda_{D^{q}}^{t} \Lambda_{D^{q}}^{*}\right)+3 \operatorname{Tr}\left(E \Lambda_{D^{q}} \Lambda_{D^{q}}^{\dagger}\right)+\operatorname{Tr}\left(E \Lambda_{E^{q}} \Lambda_{E^{q}}^{\dagger}\right)\right. \\
& \left.+6 \operatorname{Tr}\left(\tilde{D} \Lambda_{U^{q}}^{\dagger} \Lambda_{U^{q}}\right)+(\tilde{E})_{m n} \operatorname{Tr}\left(\Lambda_{E^{n}}^{\dagger} \Lambda_{E^{m}}\right)+6(\tilde{D})_{n q} \operatorname{Tr}\left(\Lambda_{D^{q}}^{\dagger} \Lambda_{D^{n}}\right)\right\} \\
& +\left(h_{D}\right)_{i l}\left\{\left(\frac{16}{3} g_{3}^{2}+\frac{16}{15} g_{1}^{2}\right)\left(\Lambda_{U^{q}} \Lambda_{U^{q}}^{\dagger}\right)_{j l}+\left(6 g_{2}^{2}+\frac{2}{5} g_{1}^{2}\right) \operatorname{Tr}\left(\Lambda_{D^{j}} \Lambda_{D^{l}}^{\dagger}\right)\right. \\
& -2\left(Y_{D}^{t} \Lambda_{D^{p}}^{\dagger} \Lambda_{D^{p}} Y_{D}^{*}\right)_{j l}-2 \operatorname{Tr}\left[\Lambda_{D^{j}}^{t} \Lambda_{D^{l}}^{*}(D+T)\right]-2 \operatorname{Tr}\left(\Lambda_{D^{l}}^{\dagger} \Lambda_{D^{j}} \Lambda_{D^{q}}^{\dagger} \Lambda_{D^{q}}\right) \\
& -6 \operatorname{Tr}\left(\Lambda_{D^{q}} \Lambda_{D^{q}}^{\dagger} \Lambda_{D^{j}} \Lambda_{D^{l}}^{\dagger}\right)-2 \operatorname{Tr}\left(\Lambda_{E^{q}} \Lambda_{E^{q}}^{\dagger} \Lambda_{D^{j}} \Lambda_{D^{l}}^{\dagger}\right)-2 \operatorname{Tr}\left(E \Lambda_{D^{j}} \Lambda_{D^{l}}^{\dagger}\right) \\
& -4\left(\Lambda_{U^{m}} \tilde{D} \Lambda_{U^{m}}^{\dagger}\right)_{j l}-4\left(\Lambda_{U^{m}}^{\dagger} \Lambda_{U^{p}} \Lambda_{U^{p}}^{\dagger} \Lambda_{U^{m}}\right)_{l j}+4\left(\Lambda_{U^{m}}^{\dagger}\right)_{l q}\left(\Lambda_{U^{m}}\right)_{j n} \operatorname{Tr}\left(\Lambda_{D^{n}} \Lambda_{D^{q}}^{\dagger}\right) \\
& -6\left(Y_{D^{\dagger}}^{\dagger} \Lambda_{D^{j}}^{t} \Lambda_{D^{p}}^{*} Y_{D}\right)_{l p}-2\left(Y_{D^{\dagger}}^{\dagger} \Lambda_{D^{j}}^{t} \Lambda_{E^{p}}^{*} Y_{E}\right)_{l p}-6\left(Y_{D}^{t} \Lambda_{D^{l}}^{\dagger} \Lambda_{D^{p}} Y_{D}^{*}\right)_{j p} \\
& \left.-2\left(Y_{D}^{t} \Lambda_{D^{l}}^{\dagger} \Lambda_{E^{p}} Y_{E}^{*}\right)_{j p}-2\left(\Lambda_{U^{q}}^{\dagger} \Lambda_{U^{n}}\right)_{l j}\left[\operatorname{Tr}\left(\Lambda_{U^{n}}^{\dagger} \Lambda_{U^{q}}\right)+2(\tilde{T})_{n q}\right]\right\} \\
& -\left(h_{D^{j}}\right)_{l i}\left\{\left(\frac{2}{5} g_{1}^{2}-16 g_{3}^{2}\right)\left(\Lambda_{D^{q}}^{*} Y_{D}\right)_{l q}-\frac{6}{5} g_{1}^{2}\left(\Lambda_{E^{q}}^{*} Y_{E}\right)_{l q}\right. \\
& -3\left(\Lambda_{E^{k}}^{\dagger} \Lambda_{D^{p}} \Lambda_{D^{p}}^{\dagger} Y_{E}\right)_{l k}-3\left(\Lambda_{E^{k}}^{\dagger} E Y_{E}\right)_{l k}-\left(\Lambda_{E^{k}}^{\dagger} \Lambda_{E^{p}} \Lambda_{E^{p}}^{\dagger} Y_{E}\right)_{l k} \\
& -\left(\Lambda_{E^{q}}^{\dagger} Y_{E}\right)_{l n} \operatorname{Tr}\left(\Lambda_{E^{n}}^{\dagger} \Lambda_{E^{q}}\right)-3\left(E^{t} \Lambda_{D^{m}}^{*} Y_{D}-E^{t} \Lambda_{E^{m}}^{*} Y_{E}\right)_{l m} \\
& +9\left(\Lambda_{D^{q}}^{*} D Y_{D}\right)_{l q}-6\left(\Lambda_{D^{q}}^{*} Y_{D} \Lambda_{U^{m}}^{\dagger} \Lambda_{U^{m}}\right)_{l q}+6\left(\Lambda_{D^{q}}^{*} Y_{D}\right)_{l n} \operatorname{Tr}\left(\Lambda_{D^{n}} \Lambda_{D^{q}}^{\dagger}\right) \\
& \left.+3\left(\Lambda_{D^{k}}^{*} T Y_{D}\right)_{l k}+3\left(\Lambda_{D^{k}}^{*} \Lambda_{D^{m}}^{t} \Lambda_{D^{m}}^{*} Y_{D}\right)_{l k}\right\} \\
& -2\left(Y_{D}\right)_{l j}\left\{\frac{2}{5} g_{1}^{2}\left[M_{1}\left(\Lambda_{D^{q}}^{\dagger} \Lambda_{D^{q}}\right)_{l i}-\left(\Lambda_{D^{q}}^{\dagger} h_{D^{q}}\right)_{l i}\right]+\left(\Lambda_{D^{m}}^{\dagger} h_{E} Y_{E}^{\dagger} \Lambda_{D^{m}}\right)_{l i}\right. \\
& +\left(Y_{D}^{*}\right)_{l n}\left(\Lambda_{D^{n}}^{t} \Lambda_{E^{m}}^{*} h_{E}\right)_{i m}+\left(h_{U}\right)_{i n}\left(Y_{U}^{\dagger}\right)_{q l} \operatorname{Tr}\left(\Lambda_{U^{n}}^{\dagger} \Lambda_{U^{q}}\right) \\
& +2\left(\Lambda_{D^{n}}^{\dagger} \Lambda_{D^{m}}\right)_{l i}\left(Y_{D}^{\dagger} h_{D}\right)_{m n}+3\left(h_{D}\right)_{i n}\left(\Lambda_{D^{n}}^{\dagger} \Lambda_{D^{m}} Y_{D}^{*}\right)_{l m} \\
& +\left(h_{D}\right)_{i n}\left(\Lambda_{D^{n}}^{\dagger} \Lambda_{E^{m}} Y_{E}^{*}\right)_{l m}+2\left(h_{D} \Lambda_{U^{q}}^{\dagger} \Lambda_{U^{q}} Y_{D}^{\dagger}\right)_{i l}+2\left(h_{D}\right)_{i m}\left(Y_{D}^{\dagger}\right)_{n l} \operatorname{Tr}\left(\Lambda_{D^{m}}^{\dagger} \Lambda_{D^{n}}\right) \\
& +3\left(Y_{D}^{*}\right)_{l n}\left(\Lambda_{D^{n}}^{t} \Lambda_{D^{m}}^{*} h_{D}\right)_{i m}+\left(\Lambda_{D^{m}}^{\dagger} h_{E^{q}} \Lambda_{E^{q}}^{\dagger} \Lambda_{D^{m}}\right)_{l i}+\left(Y_{D}\right)_{i n}\left(\Lambda_{D^{n}}^{\dagger} h_{E^{m}} Y_{E}^{*}\right)_{l m} \\
& +2\left(\Lambda_{D^{n}}^{\dagger} \Lambda_{D^{m}}\right)_{l i}\left(h_{U^{q}} \Lambda_{U^{q}}^{\dagger}\right)_{n m}+2\left(Y_{D} \Lambda_{U^{q}}^{\dagger} h_{U^{q}} Y_{D}^{\dagger}\right)_{i l}+\left(Y_{U}\right)_{i n}\left(Y_{U}^{\dagger}\right)_{q l} \operatorname{Tr}\left(\Lambda_{U^{n}}^{\dagger} h_{U^{q}}\right) \\
& +2\left(\Lambda_{D^{n}}^{\dagger} h_{D^{m}}\right)_{l i}\left[(\tilde{D})_{m n}+\left(\Lambda_{U^{q}} \Lambda_{U^{q}}^{\dagger}\right)_{n m}+\operatorname{Tr}\left(\Lambda_{D^{m}}^{\dagger} \Lambda_{D^{n}}\right)\right] \\
& +2\left(\Lambda_{D^{n}}^{\dagger} \Lambda_{D^{m}}\right)_{l i} \operatorname{Tr}\left(\Lambda_{D^{m}}^{\dagger} h_{D^{n}}\right)+\left(\Lambda_{D^{m}}^{\dagger} E h_{D^{m}}\right)_{l i}+\left(Y_{D}^{*}\right)_{l n}\left(h_{D^{n}}^{t} \Lambda_{E^{m}}^{*} Y_{E}\right)_{i m} \\
& +3\left(\Lambda_{D^{m}}^{\dagger} h_{D^{q}} \Lambda_{D^{q}}^{\dagger} \Lambda_{D^{m}}\right)_{l i}+3\left(\Lambda_{D^{m}}^{\dagger} \Lambda_{D^{q}} \Lambda_{D^{q}}^{\dagger} h_{D^{m}}\right)_{l i}+\left(\Lambda_{D^{m}}^{\dagger} \Lambda_{E^{q}} \Lambda_{E^{q}}^{\dagger} h_{D^{m}}\right)_{l i} \\
& \left.+3\left(Y_{D}\right)_{i n}\left(\Lambda_{D^{n}}^{\dagger} h_{D^{m}} Y_{D}^{*}\right)_{l m}+2\left(Y_{D}\right)_{i m}\left(Y_{D}^{\dagger}\right)_{n l} \operatorname{Tr}\left(\Lambda_{D^{m}}^{\dagger} h_{D^{n}}\right)+3\left(Y_{D}^{*}\right)_{l n}\left(h_{D^{n}}^{t} \Lambda_{D^{m}}^{*} Y_{D}\right)_{i m}\right\} \\
& -2\left(Y_{D}\right)_{i j}\left\{3 \operatorname{Tr}\left(h_{E} Y_{E}^{\dagger} \Lambda_{D^{q}} \Lambda_{D^{q}}^{\dagger}\right)+\operatorname{Tr}\left(h_{E} Y_{E^{\dagger}}^{\dagger} \Lambda_{E^{q}} \Lambda_{E^{q}}^{\dagger}\right)+\left(Y_{E}^{\dagger} h_{E}\right)_{m n} \operatorname{Tr}\left(\Lambda_{E^{m}} \Lambda_{E^{n}}^{\dagger}\right)\right. \\
& +6 \operatorname{Tr}\left(Y_{D}^{\dagger} h_{D} \Lambda_{U^{q}}^{\dagger} \Lambda_{U^{q}}\right)+3 \operatorname{Tr}\left(h_{D} Y_{D}^{\dagger} \Lambda_{D^{q}}^{t} \Lambda_{D^{q}}^{*}\right)+6\left(Y_{D}^{\dagger} h_{D}\right){ }_{l q} \operatorname{Tr}\left(\Lambda_{D^{q}}^{\dagger} \Lambda_{D^{l}}\right) \\
& +\operatorname{Tr}\left(E h_{E^{q}} \Lambda_{E^{q}}^{\dagger}\right)+(\tilde{E})_{m n} \operatorname{Tr}\left(\Lambda_{E^{n}}^{\dagger} h_{E^{m}}\right)+6 \operatorname{Tr}\left(\tilde{D} \Lambda_{U^{q}}^{\dagger} h_{U^{q}}\right) \\
& \left.+3 \operatorname{Tr}\left(E h_{D^{q}} \Lambda_{D^{q}}^{\dagger}\right)+3 \operatorname{Tr}\left(D h_{D^{q}}^{t} \Lambda_{D^{q}}^{*}\right)+6(\tilde{D})_{l q} \operatorname{Tr}\left(\Lambda_{D^{q}}^{\dagger} h_{D^{l}}\right)\right\} \\
& -2\left(Y_{D}\right)_{i l}\left\{\left(\frac{16}{3} M_{3} g_{3}^{2}+\frac{16}{15} M_{1} g_{1}^{2}\right)\left(\Lambda_{U^{q}} \Lambda_{U^{q}}^{\dagger}\right)_{j l}\right. \\
& -\left(\frac{16}{3} g_{3}^{2}+\frac{16}{15} g_{1}^{2}\right)\left(h_{U^{q}} \Lambda_{U^{q}}^{\dagger}\right)_{j l}+\left(6 M_{2} g_{2}^{2}+\frac{2}{5} M_{1} g_{1}^{2}\right) \operatorname{Tr}\left(\Lambda_{D^{j}} \Lambda_{D^{l}}^{\dagger}\right) \\
& -\left(6 g_{2}^{2}+\frac{2}{5} g_{1}^{2}\right) \operatorname{Tr}\left(h_{D^{j}} \Lambda_{D^{l}}^{\dagger}\right)+2 \operatorname{Tr}\left(h_{E} Y_{E^{\dagger}}^{\dagger} \Lambda_{D^{j}} \Lambda_{D^{l}}^{\dagger}\right)+2\left(Y_{D^{\dagger}}^{\dagger} \Lambda_{D^{j}}^{t} \Lambda_{E^{p}}^{*} h_{E}\right)_{l p} \\
& +2 \operatorname{Tr}\left(\Lambda_{D^{j}}^{t} \Lambda_{D^{l}}^{*} h_{U} Y_{U}^{\dagger}\right)+4\left(\Lambda_{U^{q}}^{\dagger} \Lambda_{U^{n}}\right)_{l j}\left(Y_{U}^{\dagger} h_{U}\right)_{n q} \\
& +2\left(h_{D}^{t} \Lambda_{D^{p}}^{\dagger} \Lambda_{D^{p}} Y_{D}^{*}\right)_{j l}+2 \operatorname{Tr}\left(\Lambda_{D^{j}}^{t} \Lambda_{D^{l}}^{*} h_{D} Y_{D}^{\dagger}\right)+4\left(\Lambda_{U^{m}} Y_{D}^{\dagger} h_{D} \Lambda_{U^{m}}^{\dagger}\right)_{j l} \\
& +6\left(Y_{D}^{\dagger} \Lambda_{D^{j}}^{t} \Lambda_{D^{p}}^{*} h_{D}\right)_{l p}+6\left(h_{D}^{t} \Lambda_{D^{l}}^{\dagger} \Lambda_{D^{p}} Y_{D}^{*}\right)_{j p}+2\left(h_{D}^{t} \Lambda_{D^{l}}^{\dagger} \Lambda_{E^{p}} Y_{E}^{*}\right)_{j p}
\end{aligned}
$$




$$
\begin{aligned}
& +2 \operatorname{Tr}\left(h_{E^{q}} \Lambda_{E^{q}}^{\dagger} \Lambda_{D^{j}} \Lambda_{D^{l}}^{\dagger}\right)+2\left(Y_{D}^{t} \Lambda_{D^{l}}^{\dagger} h_{E^{p}} Y_{E}^{*}\right)_{j p}+4\left(h_{U^{m}} \tilde{D} \Lambda_{U^{m}}^{\dagger}\right)_{j l} \\
& +4\left(\Lambda_{U^{m}}^{\dagger} h_{U^{p}} \Lambda_{U^{p}}^{\dagger} \Lambda_{U^{m}}\right)_{l j}+4\left(\Lambda_{U^{m}}^{\dagger} \Lambda_{U^{p}} \Lambda_{U^{p}}^{\dagger} h_{U^{m}}\right)_{l j} \\
& +2\left(\Lambda_{U^{q}}^{\dagger} h_{U^{n}}\right)_{l j} \operatorname{Tr}\left(\Lambda_{U^{n}}^{\dagger} \Lambda_{U^{q}}\right)+4\left(\Lambda_{U^{q}}^{\dagger} h_{U^{n}}\right)_{l j}(\tilde{T})_{n q}+2\left(\Lambda_{U^{q}}^{\dagger} \Lambda_{U^{n}}\right)_{l j} \operatorname{Tr}\left(\Lambda_{U^{n}}^{\dagger} h_{U^{q}}\right) \\
& +2\left(Y_{D}^{t} \Lambda_{D^{p}}^{\dagger} h_{D^{p}} Y_{D}^{*}\right)_{j l}+2 \operatorname{Tr}\left(h_{D^{j}}^{t} \Lambda_{D^{l}}^{*} D\right)+2 \operatorname{Tr}\left(h_{D^{j}}^{t} \Lambda_{D^{l}}^{*} T\right) \\
& +6 \operatorname{Tr}\left(h_{D^{q}} \Lambda_{D^{q}}^{\dagger} \Lambda_{D^{j}} \Lambda_{D^{l}}^{\dagger}\right)+6 \operatorname{Tr}\left(\Lambda_{D^{q}} \Lambda_{D^{q}}^{\dagger} h_{D^{j}} \Lambda_{D^{l}}^{\dagger}\right)+2 \operatorname{Tr}\left(\Lambda_{E^{q}} \Lambda_{E^{q}}^{\dagger} h_{D^{j}} \Lambda_{D^{l}}^{\dagger}\right. \\
& \left.+E h_{D^{j}} \Lambda_{D^{l}}^{\dagger}\right)-4\left(\Lambda_{U^{m}}^{\dagger}\right)_{l q}\left(\Lambda_{U^{m}}\right)_{j n} \operatorname{Tr}\left(h_{D^{n}} \Lambda_{D^{q}}^{\dagger}\right)+6\left(Y_{D^{\prime}}^{\dagger} h_{D^{j}}^{t} \Lambda_{D^{p}}^{*} Y_{D}\right)_{l p} \\
& +2\left(Y_{D^{\dagger}}^{\dagger} h_{D^{j}}^{t} \Lambda_{E^{p}}^{*} Y_{E}\right)_{l p}+6\left(Y_{D}^{t} \Lambda_{D^{l}}^{\dagger} h_{D^{p}} Y_{D}^{*}\right)_{j p}+2 \operatorname{Tr}\left(\Lambda_{D^{l}}^{\dagger} h_{D^{j}} \Lambda_{D^{q}}^{\dagger} \Lambda_{D^{q}}\right) \\
& \left.+2 \operatorname{Tr}\left(\Lambda_{D^{l}}^{\dagger} \Lambda_{D^{j}} \Lambda_{D^{q}}^{\dagger} h_{D^{q}}\right)-4\left(\Lambda_{U^{m}}^{\dagger}\right)_{l q}\left(h_{U^{m}}\right)_{j n} \operatorname{Tr}\left(\Lambda_{D^{n}} \Lambda_{D^{q}}^{\dagger}\right)\right\} \\
& +2\left(\Lambda_{D^{j}}\right)_{l i}\left\{\left(\frac{2}{5} M_{1} g_{1}^{2}-16 M_{3} g_{3}^{2}\right)\left(\Lambda_{D^{q}}^{*} Y_{D}\right)_{l q}-\frac{6}{5} M_{1} g_{1}^{2}\left(\Lambda_{E^{q}}^{*} Y_{E}\right)_{l q}\right. \\
& +\left(16 g_{3}^{2}-\frac{2}{5} g_{1}^{2}\right)\left(\Lambda_{D^{q}}^{*} h_{D}\right)_{l q}+\frac{6}{5} g_{1}^{2}\left(\Lambda_{E^{q}}^{*} h_{E}\right)_{l q}+3\left(\Lambda_{E^{q}}^{\dagger} \Lambda_{D^{p}} \Lambda_{D^{p}}^{\dagger} h_{E}\right)_{l q} \\
& +\left(\Lambda_{E^{q}}^{\dagger} \Lambda_{E^{p}} \Lambda_{E^{p}}^{\dagger} h_{E}\right)_{l q}+3\left(\Lambda_{E^{q}}^{\dagger} h_{E} \tilde{E}+\Lambda_{E^{q}}^{\dagger} E h_{E}\right)_{l q} \\
& +\left(\Lambda_{E q}^{\dagger} h_{E}\right)_{l n} \operatorname{Tr}\left(\Lambda_{E^{n}}^{\dagger} \Lambda_{E^{q}}\right)-3\left(\Lambda_{D^{q}}^{*} h_{U} Y_{U}^{\dagger} Y_{D}+\Lambda_{D^{q}}^{*} h_{D^{m}}^{t} \Lambda_{D^{m}}^{*} Y_{D}\right)_{l q} \\
& +3\left(Y_{E}^{*} h_{E}^{t} \Lambda_{D^{m}}^{*} Y_{D}-Y_{E}^{*} h_{E}^{t} \Lambda_{E m}^{*} Y_{E}-E^{t} \Lambda_{E m}^{*} h_{E}\right)_{l m} \\
& +3\left(E^{t} \Lambda_{D^{m}}^{*} h_{D}\right)_{l m}-9\left(\Lambda_{D^{q}}^{*} h_{D} \tilde{D}+\Lambda_{D^{q}}^{*} D h_{D}\right)_{l q} \\
& +6\left(\Lambda_{D^{q}}^{*} h_{D} \Lambda_{U^{m}}^{\dagger} \Lambda_{U^{m}}\right)_{l q}-6\left(\Lambda_{D^{q}}^{*} h_{D}\right)_{l n} \operatorname{Tr}\left(\Lambda_{D^{n}} \Lambda_{D^{q}}^{\dagger}\right)-3\left(\Lambda_{D^{q}}^{*} T h_{D}\right)_{l q} \\
& -3\left(\Lambda_{D^{q}}^{*} \Lambda_{D^{m}}^{t} \Lambda_{D^{m}}^{*} h_{D}\right)_{l q}+\left(\Lambda_{E^{q}}^{\dagger} h_{E^{p}} \Lambda_{E^{p}}^{\dagger} Y_{E}\right)_{l q}+\left(\Lambda_{E^{q}}^{\dagger} Y_{E}\right)_{l n} \operatorname{Tr}\left(\Lambda_{E^{n}}^{\dagger} h_{E^{q}}\right) \\
& \left.+6\left(\Lambda_{D^{q}}^{*} Y_{D} \Lambda_{U m}^{\dagger} h_{U^{m}}\right)_{l q}+3\left(\Lambda_{E q}^{\dagger} h_{D^{p}} \Lambda_{D^{p}}^{\dagger} Y_{E}\right)_{l q}-6\left(\Lambda_{D^{q}}^{*} Y_{D}\right)_{l n} \operatorname{Tr}\left(h_{D^{n}} \Lambda_{D^{q}}^{\dagger}\right)\right\} \\
& \left(16 \pi^{2}\right)^{2} \beta_{\left(h_{U}\right)_{i j}}^{(2)}=\left(16 \pi^{2}\right)^{2} \beta_{\left(h_{U}\right)_{(R P C)}}^{(2)} \\
& +\left(h_{U}\right)_{i l}\left\{\left(\frac{8}{3} g_{3}^{2}-\frac{4}{15} g_{1}^{2}\right) \operatorname{Tr}\left(\Lambda_{U^{j}} \Lambda_{U^{l}}^{\dagger}\right)-4 \operatorname{Tr}\left(\Lambda_{U^{j}} \Lambda_{U^{l}}^{\dagger} \Lambda_{U^{p}} \Lambda_{U^{p}}^{\dagger}\right)\right. \\
& -2\left(Y_{U^{\dagger}}^{\dagger} \Lambda_{D^{m}}^{t} \Lambda_{D^{m}}^{*} Y_{U}\right)_{l j}-4\left(\Lambda_{U^{l}}^{\dagger} \Lambda_{U^{j}}\right)_{n m} \operatorname{Tr}\left(\Lambda_{D^{m}}^{\dagger} \Lambda_{D^{n}}\right) \\
& \left.-4 \operatorname{Tr}\left(\Lambda_{U^{l}}^{\dagger} \Lambda_{U^{j}} \tilde{D}\right)\right\}+\left(h_{U}\right)_{l j}\left\{\frac{2}{5} g_{1}^{2}\left(\Lambda_{D^{q}}^{\dagger} \Lambda_{D^{q}}\right)_{l i}-\left(\Lambda_{D^{m}}^{\dagger} E \Lambda_{D^{m}}\right)_{l i}\right. \\
& -2\left(\Lambda_{D^{n}}^{\dagger} \Lambda_{D^{m}}\right)_{l i}\left[(\tilde{D})_{m n}+\left(\Lambda_{U^{q}} \Lambda_{U^{q}}^{\dagger}\right)_{n m}+\operatorname{Tr}\left(\Lambda_{D^{m}}^{\dagger} \Lambda_{D^{n}}\right)\right] \\
& -3\left(\Lambda_{D^{m}}^{\dagger} \Lambda_{D^{q}} \Lambda_{D^{q}}^{\dagger} \Lambda_{D^{m}}\right)_{l i}-\left(\Lambda_{D^{m}}^{\dagger} \Lambda_{E^{q}} \Lambda_{E^{q}}^{\dagger} \Lambda_{D^{m}}\right)_{l i}-\left(Y_{D}\right)_{i n}\left(3 \Lambda_{D^{n}}^{\dagger} \Lambda_{D^{m}} Y_{D}^{*}\right. \\
& \left.+\Lambda_{D^{n}}^{\dagger} \Lambda_{E^{m}} Y_{E}^{*}\right)_{l m}-2\left(Y_{D} \Lambda_{U^{q}}^{\dagger} \Lambda_{U^{q}} Y_{D}^{\dagger}\right)_{i l}-2\left(Y_{D}\right)_{i m}\left(Y_{D}^{\dagger}\right)_{n l} \operatorname{Tr}\left(\Lambda_{D^{m}}^{\dagger} \Lambda_{D^{n}}\right) \\
& \left.-\left(Y_{D}^{*}\right)_{l n}\left(3 \Lambda_{D^{n}}^{t} \Lambda_{D^{m}}^{*} Y_{D}+\Lambda_{D^{n}}^{t} \Lambda_{E^{m}}^{*} Y_{E}\right)_{i m}-\left(Y_{U}\right)_{i n}\left(Y_{U}^{\dagger}\right)_{q l} \operatorname{Tr}\left(\Lambda_{U^{n}}^{\dagger} \Lambda_{U^{q}}\right)\right\} \\
& -\left(h_{U}\right)_{i j}\left\{3 \operatorname{Tr}\left(T \Lambda_{D^{q}}^{t} \Lambda_{D^{q}}^{*}\right)+3 \tilde{T}_{l q} \operatorname{Tr}\left(\Lambda_{U^{q}}^{\dagger} \Lambda_{U^{l}}\right)\right\} \\
& -2\left(Y_{U}\right)_{i l}\left\{\left(\frac{8}{3} M_{3} g_{3}^{2}-\frac{4}{15} M_{1} g_{1}^{2}\right) \operatorname{Tr}\left(\Lambda_{U^{j}} \Lambda_{U^{l}}^{\dagger}\right)+\left(\frac{4}{15} g_{1}^{2}-\frac{8}{3} g_{3}^{2}\right) \operatorname{Tr}\left(h_{U^{j}} \Lambda_{U^{l}}^{\dagger}\right)\right. \\
& +2\left(Y_{U^{\dagger}}^{\dagger} \Lambda_{D^{m}}^{t} \Lambda_{D^{m}}^{*} h_{U}\right)_{l j}+4 \operatorname{Tr}\left(\Lambda_{U^{l}}^{\dagger} \Lambda_{U^{j}} Y_{D}^{\dagger} h_{D}\right)+4\left(\Lambda_{U^{l}}^{\dagger} h_{U^{j}}\right)_{n m} \operatorname{Tr}\left(\Lambda_{D^{m}}^{\dagger} \Lambda_{D^{n}}\right) \\
& +4 \operatorname{Tr}\left(h_{U^{j}} \Lambda_{U^{l}}^{\dagger} \Lambda_{U^{p}} \Lambda_{U^{p}}^{\dagger}\right)+4 \operatorname{Tr}\left(\Lambda_{U^{j}} \Lambda_{U^{l}}^{\dagger} h_{U^{p}} \Lambda_{U^{p}}^{\dagger}\right)+4 \operatorname{Tr}\left(\Lambda_{U^{l}}^{\dagger} h_{U^{j}} \tilde{D}\right) \\
& \left.+2\left(Y_{U^{\prime}}^{\dagger} h_{D^{m}}^{t} \Lambda_{D^{m}}^{*} Y_{U}\right)_{l j}+4\left(\Lambda_{U^{l}}^{\dagger} \Lambda_{U^{j}}\right)_{n m} \operatorname{Tr}\left(\Lambda_{D^{m}}^{\dagger} h_{D^{n}}\right)\right\} \\
& -2\left(Y_{U}\right)_{l j}\left\{\frac{2}{5} M_{1} g_{1}^{2}\left(\Lambda_{D^{q}}^{\dagger} \Lambda_{D^{q}}\right)_{l i}-\frac{2}{5} g_{1}^{2}\left(\Lambda_{D^{q}}^{\dagger} h_{D^{q}}\right)_{l i}\right. \\
& +\left(\Lambda_{D^{m}}^{\dagger} h_{E} Y_{E}^{\dagger} \Lambda_{D^{m}}\right)_{l i}+\left(Y_{D}^{*}\right)_{l n}\left(\Lambda_{D^{n}}^{t} \Lambda_{E}^{*} h_{E}\right)_{i m} \\
& +\left(h_{U}\right)_{i n}\left(Y_{U}^{\dagger}\right)_{q l} \operatorname{Tr}\left(\Lambda_{U^{n}}^{\dagger} \Lambda_{U^{q}}\right)+2\left(\Lambda_{D^{n}}^{\dagger} \Lambda_{D^{m}}\right)_{l i}\left(Y_{D^{\dagger}}^{\dagger} h_{D}\right)_{m n} \\
& +\left(h_{D}\right)_{i n}\left(3 \Lambda_{D^{n}}^{\dagger} \Lambda_{D^{m}} Y_{D}^{*}+\Lambda_{D^{n}}^{\dagger} \Lambda_{E^{m}} Y_{E}^{*}\right)_{l m}+2\left(h_{D} \Lambda_{U^{q}}^{\dagger} \Lambda_{U^{q}} Y_{D}^{\dagger}\right)_{i l}
\end{aligned}
$$




$$
\begin{aligned}
& +2\left(h_{D}\right)_{i m}\left(Y_{D}^{\dagger}\right)_{n l} \operatorname{Tr}\left(\Lambda_{D^{m}}^{\dagger} \Lambda_{D^{n}}\right)+3\left(Y_{D}^{*}\right)_{l n}\left(\Lambda_{D^{n}}^{t} \Lambda_{D^{m}}^{*} h_{D}\right)_{i m} \\
& +\left(Y_{D}\right)_{i n}\left(\Lambda_{D^{n}}^{\dagger} h_{E^{m}} Y_{E}^{*}\right)_{l m}+2\left(\Lambda_{D^{n}}^{\dagger} \Lambda_{D^{m}}\right)_{l i}\left(h_{U^{q}} \Lambda_{U^{q}}^{\dagger}\right)_{n m}+2\left(Y_{D} \Lambda_{U^{q}}^{\dagger} h_{U^{q}} Y_{D}^{\dagger}\right)_{i l} \\
& +\left(Y_{U}\right)_{i n}\left(Y_{U}^{\dagger}\right)_{q l} \operatorname{Tr}\left(\Lambda_{U^{n}}^{\dagger} h_{U^{q}}\right)+\left(\Lambda_{D^{m}}^{\dagger} h_{E^{q}} \Lambda_{E^{q}}^{\dagger} \Lambda_{D^{m}}\right)_{l i} \\
& +2\left(\Lambda_{D^{n}}^{\dagger} h_{D^{m}}\right)_{l i}\left[(\tilde{D})_{m n}+\left(\Lambda_{U^{q}} \Lambda_{U^{q}}^{\dagger}\right)_{n m}+\operatorname{Tr}\left(\Lambda_{D^{m}}^{\dagger} \Lambda_{D^{n}}\right)\right] \\
& +2\left(\Lambda_{D^{n}}^{\dagger} \Lambda_{D^{m}}\right)_{l i} \operatorname{Tr}\left(\Lambda_{D^{m}}^{\dagger} h_{D^{n}}\right)+\left(\Lambda_{D^{m}}^{\dagger} E h_{D^{m}}\right)_{l i}+3\left(\Lambda_{D^{m}}^{\dagger} h_{D^{q}} \Lambda_{D^{q}}^{\dagger} \Lambda_{D^{m}}\right)_{l i} \\
& +3\left(\Lambda_{D^{m}}^{\dagger} \Lambda_{D^{q}} \Lambda_{D^{q}}^{\dagger} h_{D^{m}}\right)_{l i}+\left(\Lambda_{D^{m}}^{\dagger} \Lambda_{E^{q}} \Lambda_{E^{q}}^{\dagger} h_{D^{m}}\right)_{l i}+3\left(Y_{D}\right)_{i n}\left(\Lambda_{D^{n}}^{\dagger} h_{D^{m}} Y_{D}^{*}\right)_{l m} \\
& +2\left(Y_{D}\right)_{i m}\left(Y_{D}^{\dagger}\right)_{n l} \operatorname{Tr}\left(\Lambda_{D^{m}}^{\dagger} h_{D^{n}}\right)+3\left(Y_{D}^{*}\right)_{l n}\left(h_{D^{n}}^{t} \Lambda_{D^{m}}^{*} Y_{D}\right)_{i m} \\
& \left.+\left(Y_{D}^{*}\right)_{l n}\left(h_{D^{n}}^{t} \Lambda_{E^{m}}^{*} Y_{E}\right)_{i m}\right\}-6\left(Y_{U}\right)_{i j}\left\{(\tilde{T})_{l q} \operatorname{Tr}\left(\Lambda_{U^{q}}^{\dagger} h_{U^{l}}\right)+\operatorname{Tr}\left(T h_{D^{q}}^{t} \Lambda_{D^{q}}^{*}\right)\right. \\
& \left.+\operatorname{Tr}\left(h_{U} Y_{U}^{\dagger} \Lambda_{D^{q}}^{t} \Lambda_{D^{q}}^{*}\right)+\left(Y_{U}^{\dagger} h_{U}\right)_{l q} \operatorname{Tr}\left(\Lambda_{U^{q}}^{\dagger} \Lambda_{U^{l}}\right)\right\} \\
& \left(16 \pi^{2}\right)^{2} \beta_{\left(h_{E^{k}}\right)_{i j}}^{(2)}=\left(h_{E^{l}}\right)_{i j}\left\{\left(6 g_{2}^{2}-\frac{6}{5} g_{1}^{2}\right)(\tilde{E})_{l k}+\left(3 g_{2}^{2}-\frac{3}{5} g_{1}^{2}\right) \operatorname{Tr}\left(\Lambda_{E^{k}} \Lambda_{E^{l}}^{\dagger}\right)\right. \\
& -2\left(\tilde{E}^{2}+Y_{E}^{\dagger} \Lambda_{E^{q}} \Lambda_{E^{q}}^{\dagger} Y_{E}+3 Y_{E}^{\dagger} \Lambda_{D^{q}} \Lambda_{D^{q}}^{\dagger} Y_{E}\right)_{l k} \\
& -2(\tilde{E})_{l k} \operatorname{Tr}(E+3 D)+2\left(3 Y_{E}^{\dagger} \Lambda_{E^{k}} \Lambda_{D}^{*} Y_{D}\right. \\
& \left.-Y_{E}^{\dagger} \Lambda_{E^{k}} \Lambda_{E^{m}}^{\dagger} Y_{E}\right)_{l m}-2 \operatorname{Tr}\left[\Lambda_{E^{k}} \Lambda_{E^{l}}^{\dagger}\left(E+\Lambda_{E^{q}} \Lambda_{E^{q}}^{\dagger}+3 \Lambda_{D^{q}} \Lambda_{D^{q}}^{\dagger}\right)\right] \\
& \left.+2\left(3 Y_{D}^{\dagger} \Lambda_{D^{m}}^{t} \Lambda_{E l}^{\dagger} Y_{E}-Y_{E}^{\dagger} \Lambda_{E^{m}} \Lambda_{E l}^{\dagger} Y_{E}\right)_{m k}\right\} \\
& +\left(h_{E}\right)_{i k}\left\{\left(\frac{2}{5} g_{1}^{2}-16 g_{3}^{2}\right)\left(\Lambda_{D^{q}} Y_{D}^{*}\right)_{j q}+6\left(\Lambda_{D^{q}} Y_{D}^{*}\right)_{j l} \operatorname{Tr}\left(\Lambda_{D^{l}}^{*} \Lambda_{D^{q}}^{t}\right)\right. \\
& -\left(3 \Lambda_{E q}^{t} \Lambda_{D^{p}}^{*} \Lambda_{D p}^{t} Y_{E}^{*}+\Lambda_{E q}^{t} \Lambda_{E p}^{*} \Lambda_{E p}^{t} Y_{E}^{*}+3 \Lambda_{E q}^{t} E^{t} Y_{E}^{*}\right)_{j q} \\
& -\left(Y_{E}^{\dagger} \Lambda_{E^{q}}\right)_{l j} \operatorname{Tr}\left(\Lambda_{E^{q}}^{\dagger} \Lambda_{E^{l}}\right)-3\left(E \Lambda_{D^{m}} Y_{D}^{*}-E \Lambda_{E^{m}} Y_{E}^{*}\right)_{j m} \\
& +9\left(\Lambda_{D^{q}} D^{t} Y_{D}^{*}\right)_{j q}-6\left(\Lambda_{D^{q}} Y_{D}^{*} \Lambda_{U^{m}}^{t} \Lambda_{U^{m}}^{*}\right)_{j q}-\frac{6}{5} g_{1}^{2}\left(\Lambda_{E^{q}} Y_{E}^{*}\right)_{j q} \\
& \left.+3\left(\Lambda_{D^{q}} Y_{U}^{*} Y_{U}^{t} Y_{D}^{*}\right)_{j q}+3\left(\Lambda_{D^{q}} \Lambda_{D^{m}}^{\dagger} \Lambda_{D^{m}} Y_{D}^{*}\right)_{j q}\right\} \\
& +\left(h_{E^{k}}\right)_{l j}\left\{\left(16 g_{3}^{2}-\frac{2}{5} g_{1}^{2}\right)\left(\Lambda_{D^{q}} \Lambda_{D^{q}}^{\dagger}\right)_{i l}+\frac{6}{5} g_{1}^{2}\left(E+\Lambda_{E^{q}} \Lambda_{E^{q}}^{\dagger}\right)_{i l}\right. \\
& -2\left(E^{2}\right)_{i l}-\left(Y_{E}^{\dagger}\right)_{n l}\left(Y_{E}\right)_{i m} \operatorname{Tr}\left(\Lambda_{E^{m}}^{\dagger} \Lambda_{E^{n}}\right)-2\left(\Lambda_{E^{m}} \Lambda_{E^{n}}^{\dagger}\right)_{i l}(\tilde{E})_{m n} \\
& -\left(\Lambda_{E^{m}} \Lambda_{E^{n}}^{\dagger}\right)_{i l} \operatorname{Tr}\left(\Lambda_{E^{m}}^{\dagger} \Lambda_{E^{n}}\right)-(E)_{i l} \operatorname{Tr}(E+3 D) \\
& -\left(Y_{E}\right)_{i n}\left(3 \Lambda_{E^{n}}^{\dagger} \Lambda_{D^{p}} Y_{D}^{*}+\Lambda_{E^{n}}^{\dagger} \Lambda_{E^{p}} Y_{E}^{*}\right)_{l p}+\left(Y_{E}^{*}\right)_{l n}\left(3 \Lambda_{E^{n}} \Lambda_{D^{p}}^{*} Y_{D}\right. \\
& \left.+\Lambda_{E^{n}} \Lambda_{E^{p}}^{*} Y_{E}\right)_{i p}-\left(\Lambda_{E^{n}}^{\dagger} E \Lambda_{E^{n}}+3 \Lambda_{E^{n}}^{\dagger} \Lambda_{D^{p}} \Lambda_{D^{p}}^{\dagger} \Lambda_{E^{n}}+\Lambda_{E^{n}}^{\dagger} \Lambda_{E^{p}} \Lambda_{E^{p}}^{\dagger} \Lambda_{E^{n}}\right)_{l i} \\
& -6\left(\Lambda_{D^{m}} \Lambda_{D^{n}}^{\dagger}\right)_{i l}\left[(\tilde{D})_{m n}+\operatorname{Tr}\left(\Lambda_{D^{m}}^{\dagger} \Lambda_{D^{n}}\right)+\left(\Lambda_{U^{q}} \Lambda_{U^{q}}^{\dagger}\right)_{n m}\right] \\
& \left.-3\left(\Lambda_{D^{n}}^{*} D \Lambda_{D^{n}}^{t}+\Lambda_{D^{n}}^{*} T \Lambda_{D^{n}}^{t}\right)_{l i}-3\left(\Lambda_{D^{n}} \Lambda_{D^{p}}^{\dagger} \Lambda_{D^{p}} \Lambda_{D^{n}}^{\dagger}\right)_{i l}\right\} \\
& -\left(h_{E}\right)_{j k}\left\{\left(\frac{2}{5} g_{1}^{2}-16 g_{3}^{2}\right)\left(\Lambda_{D^{q}} Y_{D}^{*}\right)_{i q}-\frac{6}{5} g_{1}^{2}\left(\Lambda_{E^{q}} Y_{E}^{*}\right)_{i q}\right. \\
& -\left(3 \Lambda_{E^{n}}^{t} \Lambda_{D^{p}}^{*} \Lambda_{D^{p}}^{t} Y_{E}^{*}+\Lambda_{E^{n}}^{t} \Lambda_{E^{p}}^{*} \Lambda_{E^{p}}^{t} Y_{E}^{*}+3 \Lambda_{E^{n}}^{t} E^{t} Y_{E}^{*}\right)_{i n} \\
& -3\left(E \Lambda_{D^{m}} Y_{D}^{*}-E \Lambda_{E^{m}} Y_{E}^{*}\right)_{i m}+9\left(\Lambda_{D^{q}} D^{t} Y_{D}^{*}\right)_{i q} \\
& -6\left(\Lambda_{D^{q}} Y_{D}^{*} \Lambda_{U^{m}}^{t} \Lambda_{U^{m}}^{*}\right)_{i q}+6\left(\Lambda_{D^{q}} Y_{D}^{*}\right)_{i l} \operatorname{Tr}\left(\Lambda_{D^{l}}^{*} \Lambda_{D^{q}}^{t}\right) \\
& \left.+3\left(\Lambda_{D^{n}} Y_{U}^{*} Y_{U}^{t} Y_{D}^{*}\right)_{i n}+3\left(\Lambda_{D^{n}} \Lambda_{D^{m}}^{\dagger} \Lambda_{D^{m}} Y_{D}^{*}\right)_{i n}-\left(\Lambda_{E^{p}}^{t} Y_{E}^{*}\right)_{i l} \operatorname{Tr}\left(\Lambda_{E^{l}}^{t} \Lambda_{E^{p}}^{*}\right)\right\} \\
& +\left(h_{E^{k}}\right)_{i l}\left\{\left(16 g_{3}^{2}-\frac{2}{5} g_{1}^{2}\right)\left(\Lambda_{D^{q}} \Lambda_{D^{q}}^{\dagger}\right)_{j l}+\frac{6}{5} g_{1}^{2}\left(E+\Lambda_{E q} \Lambda_{E^{q}}^{\dagger}\right)_{j l}\right. \\
& -2\left(E^{2}\right)_{j l}-\left(Y_{E}^{\dagger}\right)_{n l}\left(Y_{E}\right)_{j m} \operatorname{Tr}\left(\Lambda_{E^{m}}^{\dagger} \Lambda_{E^{n}}\right)-2\left(\Lambda_{E^{m}} \Lambda_{E^{n}}^{\dagger}\right)_{j l}(\tilde{E})_{m n} \\
& -\left(\Lambda_{E^{m}} \Lambda_{E^{n}}^{\dagger}\right)_{j l} \operatorname{Tr}\left(\Lambda_{E^{m}}^{\dagger} \Lambda_{E^{n}}\right)-E_{j l} \operatorname{Tr}(E+3 D) \\
& -\left(Y_{E}\right)_{j n}\left(3 \Lambda_{E^{n}}^{\dagger} \Lambda_{D^{p}} Y_{D}^{*}+\Lambda_{E^{n}}^{\dagger} \Lambda_{E^{p}} Y_{E}^{*}\right)_{l p}+\left(Y_{E}^{*}\right)_{l n}\left(3 \Lambda_{E^{n}} \Lambda_{D^{p}}^{*} Y_{D}\right.
\end{aligned}
$$




$$
\begin{aligned}
& \left.+\Lambda_{E^{n}} \Lambda_{E^{p}}^{*} Y_{E}\right)_{j p}-\left(\Lambda_{E^{n}}^{\dagger} E \Lambda_{E^{n}}+3 \Lambda_{E^{n}}^{\dagger} \Lambda_{D^{p}} \Lambda_{D^{p}}^{\dagger} \Lambda_{E^{n}}+\Lambda_{E^{n}}^{\dagger} \Lambda_{E^{p}} \Lambda_{E^{p}}^{\dagger} \Lambda_{E^{n}}\right)_{l j} \\
& -6\left(\Lambda_{D^{m}} \Lambda_{D^{n}}^{\dagger}\right)_{j l}\left[(\tilde{D})_{m n}+\operatorname{Tr}\left(\Lambda_{D^{m}}^{\dagger} \Lambda_{D^{n}}\right)+\left(\Lambda_{U^{q}} \Lambda_{U^{q}}^{\dagger}\right)_{n m}\right] \\
& \left.-3\left(\Lambda_{D^{n}}^{*} D \Lambda_{D^{n}}^{t}+\Lambda_{D^{n}}^{*} T \Lambda_{D^{n}}^{t}\right)_{l j}-3\left(\Lambda_{D^{n}} \Lambda_{D^{p}}^{\dagger} \Lambda_{D^{p}} \Lambda_{D^{n}}^{\dagger}\right)_{j l}\right\} \\
& -2\left(\Lambda_{E^{l}}\right)_{i j}\left\{\left(6 M_{2} g_{2}^{2}-\frac{6}{5} M_{1} g_{1}^{2}\right)(\tilde{E})_{l k}+\left(3 M_{2} g_{2}^{2}-\frac{3}{5} M_{1} g_{1}^{2}\right) \operatorname{Tr}\left(\Lambda_{E^{k}} \Lambda_{E^{l}}^{\dagger}\right)\right. \\
& -\left(6 g_{2}^{2}-\frac{6}{5} g_{1}^{2}\right)\left(Y_{E}^{\dagger} h_{E}\right)_{l k}-\left(3 g_{2}^{2}-\frac{3}{5} g_{1}^{2}\right) \operatorname{Tr}\left(h_{E^{k}} \Lambda_{E^{l}}^{\dagger}\right)+2\left(Y_{E}^{\dagger} h_{E} \tilde{E}\right. \\
& \left.+Y_{E}^{\dagger} E h_{E}+Y_{E}^{\dagger} \Lambda_{E q} \Lambda_{E^{q}}^{\dagger} h_{E}+3 Y_{E}^{\dagger} \Lambda_{D^{q}} \Lambda_{D^{q}}^{\dagger} h_{E}\right)_{l k} \\
& +2\left(Y_{E}^{\dagger} h_{E}\right)_{l k} \operatorname{Tr}(E+3 D)+2(\tilde{E})_{l k} \operatorname{Tr}\left(Y_{E}^{\dagger} h_{E}\right) \\
& +2\left(Y_{E^{\dagger}}^{\dagger} \Lambda_{E^{k}} \Lambda_{E^{m}}^{\dagger} h_{E}\right)_{l m}+2 \operatorname{Tr}\left(\Lambda_{E^{k}} \Lambda_{E^{l}}^{\dagger} h_{E} Y_{E}^{\dagger}\right) \\
& -2\left(3 Y_{D}^{\dagger} \Lambda_{D^{m}}^{t} \Lambda_{E^{l}}^{\dagger} h_{E}-Y_{E}^{\dagger} \Lambda_{E^{m}} \Lambda_{E^{l}}^{\dagger} h_{E}\right)_{m k}+6(\tilde{E})_{l k} \operatorname{Tr}\left(Y_{D}^{\dagger} h_{D}\right) \\
& +2\left(Y_{E}^{\dagger} h_{E^{k}} \Lambda_{E^{m}}^{\dagger} Y_{E}\right)_{l m}-6\left(Y_{E^{\prime}}^{\dagger} \Lambda_{E^{k}} \Lambda_{D^{m}}^{*} h_{D}\right)_{l m}+2\left(Y_{E^{\prime}}^{\dagger} h_{E^{q}} \Lambda_{E^{q}}^{\dagger} Y_{E}\right)_{l k} \\
& -6\left(Y_{E}^{\dagger} h_{E^{k}} \Lambda_{D^{m}}^{*} Y_{D}\right)_{l m}+2 \operatorname{Tr}\left(h_{E^{k}} \Lambda_{E^{l}}^{\dagger} E\right)+6 \operatorname{Tr}\left(h_{E^{k}} \Lambda_{E^{l}}^{\dagger} \Lambda_{D^{q}} \Lambda_{D^{q}}^{\dagger}\right) \\
& +2 \operatorname{Tr}\left(h_{E^{k}} \Lambda_{E^{l}}^{\dagger} \Lambda_{E^{q}} \Lambda_{E^{q}}^{\dagger}\right)+2 \operatorname{Tr}\left(\Lambda_{E^{k}} \Lambda_{E^{l}}^{\dagger} h_{E^{q}} \Lambda_{E^{q}}^{\dagger}\right)+2\left(Y_{E}^{\dagger} h_{E^{m}} \Lambda_{E^{l}}^{\dagger} Y_{E}\right)_{m k} \\
& \left.+6\left(Y_{E}^{\dagger} h_{D^{q}} \Lambda_{D^{q}}^{\dagger} Y_{E}\right)_{l k}+6 \operatorname{Tr}\left(\Lambda_{E^{k}} \Lambda_{E^{l}}^{\dagger} h_{D^{q}} \Lambda_{D^{q}}^{\dagger}\right)-6\left(Y_{D^{\dagger}}^{\dagger} h_{D^{m}}^{t} \Lambda_{E^{l}}^{\dagger} Y_{E}\right)_{m k}\right\} \\
& -2\left(Y_{E}\right)_{i k}\left\{\left(\frac{2}{5} M_{1} g_{1}^{2}-16 M_{3} g_{3}^{2}\right)\left(\Lambda_{D^{q}} Y_{D}^{*}\right)_{j q}-\frac{6}{5} M_{1} g_{1}^{2}\left(\Lambda_{E^{q}} Y_{E}^{*}\right)_{j q}\right. \\
& -\left(\frac{2}{5} g_{1}^{2}-16 g_{3}^{2}\right)\left(h_{D^{q}} Y_{D}^{*}\right)_{j q}+\frac{6}{5} g_{1}^{2}\left(h_{E q} Y_{E}^{*}\right)_{j q}+\left(3 \Lambda_{E^{n}}^{t} Y_{E}^{*} h_{E}^{t} Y_{E}^{*}\right)_{j n} \\
& +3\left(h_{E} Y_{E}^{\dagger} \Lambda_{D^{m}} Y_{D}^{*}-h_{E} Y_{E}^{\dagger} \Lambda_{E^{m}} Y_{E}^{*}\right)_{j m}-3\left(\Lambda_{D^{n}} Y_{U}^{*} h_{U}^{t} Y_{D}^{*}\right)_{j n} \\
& -9\left(\Lambda_{D^{q}} Y_{D}^{*} h_{D}^{t} Y_{D}^{*}\right)_{j q}+3\left(h_{E^{n}}^{t} \Lambda_{D^{p}}^{*} \Lambda_{D^{p}}^{t} Y_{E}^{*}+h_{E^{n}}^{t} E^{t} Y_{E}^{*}\right)_{j n} \\
& +\left(h_{E^{n}}^{t} \Lambda_{E^{p}}^{*} \Lambda_{E^{p}}^{t} Y_{E}^{*}+\Lambda_{E^{n}}^{t} \Lambda_{E^{p}}^{*} h_{E^{p}}^{t} Y_{E}^{*}\right)_{j n}+3\left(\Lambda_{E^{n}}^{t} \Lambda_{D^{p}}^{*} h_{D^{p}}^{t} Y_{E}^{*}\right)_{j n} \\
& +\left(h_{E^{q}}^{t} Y_{E}^{*}\right)_{j l} \operatorname{Tr}\left(\Lambda_{E^{l}}^{t} \Lambda_{E^{q}}^{*}\right)+\left(Y_{E}^{\dagger} \Lambda_{E^{q}}\right)_{l j} \operatorname{Tr}\left(h_{E^{l}}^{t} \Lambda_{E^{q}}^{*}\right)-3\left(E h_{E^{m}} Y_{E}^{*}\right)_{j m} \\
& +6\left(\Lambda_{D^{q}} Y_{D}^{*} h_{U^{m}}^{t} \Lambda_{U^{m}}^{*}\right)_{j q}+3\left(E h_{D^{m}} Y_{D}^{*}\right)_{j m}-9\left(h_{D^{q}} D^{t} Y_{D}^{*}\right)_{j q} \\
& +6\left(h_{D^{q}} Y_{D}^{*} \Lambda_{U^{m}}^{t} \Lambda_{U^{m}}^{*}\right)_{j q}-6\left(h_{D^{q}} Y_{D}^{*}\right)_{j l} \operatorname{Tr}\left(\Lambda_{D^{l}}^{*} \Lambda_{D^{q}}^{t}\right)-6\left(\Lambda_{D^{q}} Y_{D}^{*}\right)_{j l} \operatorname{Tr}\left(\Lambda_{D^{l}}^{*} h_{D^{q}}^{t}\right) \\
& \left.-3\left(Y_{D}^{\dagger} T h_{D^{n}}^{t}\right)_{n j}-3\left(h_{D^{n}} \Lambda_{D^{m}}^{\dagger} \Lambda_{D^{m}} Y_{D}^{*}+\Lambda_{D^{n}} \Lambda_{D^{m}}^{\dagger} h_{D^{m}} Y_{D}^{*}\right)_{j n}\right\} \\
& +2\left(Y_{E}\right)_{j k}\left\{\left(\frac{2}{5} M_{1} g_{1}^{2}-16 M_{3} g_{3}^{2}\right)\left(\Lambda_{D^{q}} Y_{D}^{*}\right)_{i q}-\frac{6}{5} M_{1} g_{1}^{2}\left(\Lambda_{E^{q}} Y_{E}^{*}\right)_{i q}\right. \\
& -\left(\frac{2}{5} g_{1}^{2}-16 g_{3}^{2}\right)\left(h_{D^{q}} Y_{D}^{*}\right)_{i q}+\frac{6}{5} g_{1}^{2}\left(h_{E} q Y_{E}^{*}\right)_{i q}+\left(3 \Lambda_{E^{n}}^{t} Y_{E}^{*} h_{E}^{t} Y_{E}^{*}\right)_{i n} \\
& +3\left(h_{E} Y_{E}^{\dagger} \Lambda_{D^{m}} Y_{D}^{*}-h_{E} Y_{E}^{\dagger} \Lambda_{E^{m}} Y_{E}^{*}\right)_{i m}-3\left(\Lambda_{D^{n}} Y_{U}^{*} h_{U}^{t} Y_{D}^{*}\right)_{i n} \\
& -9\left(\Lambda_{D^{k}} Y_{D}^{*} h_{D}^{t} Y_{D}^{*}\right)_{i k}+3\left(h_{E^{n}}^{t} \Lambda_{D^{p}}^{*} \Lambda_{D^{p}}^{t} Y_{E}^{*}+h_{E^{n}}^{t} E^{t} Y_{E}^{*}\right)_{i n} \\
& +\left(h_{E^{n}}^{t} \Lambda_{E^{p}}^{*} \Lambda_{E^{p}}^{t} Y_{E}^{*}+\Lambda_{E^{n}}^{t} \Lambda_{E^{p}}^{*} h_{E^{p}}^{t} Y_{E}^{*}\right)_{i n}+\left(h_{E^{k}}^{t} Y_{E}^{*}\right)_{i l} \operatorname{Tr}\left(\Lambda_{E^{l}}^{t} \Lambda_{E^{k}}^{*}\right) \\
& +\left(\Lambda_{E^{k}}^{t} Y_{E}^{*}\right)_{i l} \operatorname{Tr}\left(h_{E^{l}}^{t} \Lambda_{E^{k}}^{*}\right)-3\left(E h_{E^{m}} Y_{E}^{*}\right)_{i m}+6\left(\Lambda_{D^{q}} Y_{D}^{*} h_{U^{m}}^{t} \Lambda_{U^{m}}^{*}\right)_{i q} \\
& +3\left(\Lambda_{E^{n}}^{t} \Lambda_{D^{p}}^{*} h_{D^{p}}^{t} Y_{E}^{*}\right)_{i n}+3\left(E h_{D^{m}} Y_{D}^{*}\right)_{i m}-9\left(h_{D^{q}} D^{t} Y_{D}^{*}\right)_{i q} \\
& +6\left(h_{D^{q}} Y_{D}^{*} \Lambda_{U^{m}}^{t} \Lambda_{U^{m}}^{*}\right)_{i q}-6\left(h_{D^{q}} Y_{D}^{*}\right)_{i l} \operatorname{Tr}\left(\Lambda_{D^{l}}^{*} \Lambda_{D^{q}}^{t}\right)-6\left(\Lambda_{D^{q}} Y_{D}^{*}\right)_{i l} \operatorname{Tr}\left(\Lambda_{D^{l}}^{*} h_{D^{q}}^{t}\right) \\
& \left.-3\left(h_{D^{n}} Y_{U}^{*} Y_{U}^{t} Y_{D}^{*}\right)_{i n}-3\left(h_{D^{n}} \Lambda_{D^{m}}^{\dagger} \Lambda_{D^{m}} Y_{D}^{*}+\Lambda_{D^{n}} \Lambda_{D^{m}}^{\dagger} h_{D^{m}} Y_{D}^{*}\right)_{i n}\right\} \\
& -2\left(\Lambda_{E^{k}}\right)_{i l}\left\{\left(16 M_{3} g_{3}^{2}-\frac{2}{5} M_{1} g_{1}^{2}\right)\left(\Lambda_{D^{q}} \Lambda_{D^{q}}^{\dagger}\right)_{j l}+\frac{6}{5} M_{1} g_{1}^{2}\left(E+\Lambda_{E^{q}} \Lambda_{E^{q}}^{\dagger}\right)_{j l}\right. \\
& -\left(16 g_{3}^{2}-\frac{2}{5} g_{1}^{2}\right)\left(h_{D^{q}} \Lambda_{D^{q}}^{\dagger}\right)_{j l}-\frac{6}{5} g_{1}^{2}\left(h_{E} Y_{E}^{\dagger}+h_{E^{q}} \Lambda_{E^{q}}^{\dagger}\right)_{j l} \\
& +2\left(h_{E} Y_{E}^{\dagger} E+E h_{E} Y_{E}^{\dagger}\right)_{j l}+\left(Y_{E}^{\dagger}\right)_{q l}\left(h_{E}\right)_{j n} \operatorname{Tr}\left(\Lambda_{E^{n}}^{\dagger} \Lambda_{E} q\right)
\end{aligned}
$$




$$
\begin{aligned}
& +2\left(\Lambda_{E^{n}} \Lambda_{E^{q}}^{\dagger}\right)_{j l}\left(Y_{E}^{\dagger} h_{E}\right)_{n q}+\left(h_{E} Y_{E}^{\dagger}\right)_{j l} \operatorname{Tr}(E+3 D) \\
& +(E)_{j l} \operatorname{Tr}\left(h_{E} Y_{E}^{\dagger}\right)+\left(h_{E}\right)_{j q}\left(3 \Lambda_{E q}^{\dagger} \Lambda_{D^{p}} Y_{D}^{*}+\Lambda_{E q}^{\dagger} \Lambda_{E^{p}} Y_{E}^{*}\right)_{l p} \\
& -\left(Y_{E}^{*}\right)_{l q}\left(\Lambda_{E^{q}} \Lambda_{E^{p}}^{*} h_{E}\right)_{j p}+\left(\Lambda_{E^{q}}^{\dagger} h_{E} Y_{E}^{\dagger} \Lambda_{E^{q}}\right)_{l j}+3\left(\Lambda_{D^{q}}^{*} h_{U} Y_{U}^{\dagger} \Lambda_{D^{q}}^{t}\right)_{l j} \\
& +3(E)_{j l} \operatorname{Tr}\left(h_{D} Y_{D}^{\dagger}\right)-3\left(Y_{E}^{\dagger}\right)_{q l}\left(\Lambda_{E^{q}} \Lambda_{D^{p}}^{*} h_{D}\right)_{j p}+6\left(\Lambda_{D^{n}} \Lambda_{D^{q}}^{\dagger}\right)_{j l}\left(Y_{D}^{\dagger} h_{D}\right)_{n q} \\
& +3\left(\Lambda_{D^{q}}^{*} h_{D} Y_{D}^{\dagger} \Lambda_{D^{q}}^{t}\right)_{l j}+\left(Y_{E}^{\dagger}\right)_{q l}\left(Y_{E}\right)_{j n} \operatorname{Tr}\left(\Lambda_{E^{n}}^{\dagger} h_{E^{q}}\right)+2\left(h_{E^{n}} \Lambda_{E q}^{\dagger}\right)_{j l}(\tilde{E})_{n q} \\
& +\left(h_{E^{n}} \Lambda_{E^{q}}^{\dagger}\right)_{j l} \operatorname{Tr}\left(\Lambda_{E^{n}}^{\dagger} \Lambda_{E^{q}}\right)+\left(\Lambda_{E^{n}} \Lambda_{E^{q}}^{\dagger}\right)_{j l} \operatorname{Tr}\left(\Lambda_{E^{n}}^{\dagger} h_{E^{q}}\right)+\left(Y_{E}\right)_{j q}\left(\Lambda_{E^{q}}^{\dagger} h_{E^{p}} Y_{E}^{*}\right)_{l p} \\
& -\left(Y_{E}^{\dagger}\right)_{q l}\left(3 h_{E^{q}} \Lambda_{D^{p}}^{*} Y_{D}+h_{E^{q}} \Lambda_{E^{p}}^{*} Y_{E}\right)_{j p}+\left(\Lambda_{E^{q}}^{\dagger} E h_{E^{q}}\right. \\
& \left.+3 \Lambda_{E^{q}}^{t} \Lambda_{D^{p}} \Lambda_{D^{p}}^{\dagger} h_{E^{q}}\right)_{l j}+\left(\Lambda_{E^{q}}^{\dagger} h_{E^{p}} \Lambda_{E^{p}}^{\dagger} \Lambda_{E^{q}}+\Lambda_{E^{q}}^{\dagger} \Lambda_{E^{p}} \Lambda_{E^{p}}^{\dagger} h_{E^{q}}\right)_{l j} \\
& +6\left(\Lambda_{D^{n}} \Lambda_{D^{q}}^{\dagger}\right)_{j l}\left(h_{U^{m}} \Lambda_{U^{m}}^{\dagger}\right)_{q n}+3\left(Y_{E}\right)_{j q}\left(\Lambda_{E^{q}}^{\dagger} h_{D^{p}} Y_{D}^{*}\right)_{l p}+3\left(\Lambda_{E^{q}}^{\dagger} h_{D^{p}} \Lambda_{D^{p}}^{\dagger} \Lambda_{E^{q}}\right)_{l j} \\
& +6\left(h_{D^{n}} \Lambda_{D^{q}}^{\dagger}\right)_{j l}\left[(\tilde{D})_{n q}+\operatorname{Tr}\left(\Lambda_{D^{n}}^{\dagger} \Lambda_{D^{q}}\right)+\left(\Lambda_{U^{m}} \Lambda_{U^{m}}^{\dagger}\right)_{q n}\right] \\
& +6\left(\Lambda_{D^{n}} \Lambda_{D^{q}}^{\dagger}\right)_{j l} \operatorname{Tr}\left(\Lambda_{D^{n}}^{\dagger} h_{D^{q}}\right)+3\left(\Lambda_{D^{q}}^{*} D h_{D^{q}}^{t}+\Lambda_{D^{q}}^{*} T h_{D^{q}}^{t}\right)_{l j} \\
& \left.+3\left(\Lambda_{D^{q}} \Lambda_{D^{p}}^{\dagger} h_{D^{p}} \Lambda_{D^{q}}^{\dagger}+h_{D^{q}} \Lambda_{D^{p}}^{\dagger} \Lambda_{D^{p}} \Lambda_{D^{q}}^{\dagger}\right)_{j l}\right\} \\
& -2\left(\Lambda_{E^{k}}\right)_{l j}\left\{\left(16 M_{3} g_{3}^{2}-\frac{2}{5} M_{1} g_{1}^{2}\right)\left(\Lambda_{D^{q}} \Lambda_{D^{q}}^{\dagger}\right)_{i l}+\frac{6}{5} M_{1} g_{1}^{2}\left(E+\Lambda_{E^{q}} \Lambda_{E^{q}}^{\dagger}\right)_{i l}\right. \\
& +\left(\frac{2}{5} g_{1}^{2}-16 g_{3}^{2}\right)\left(h_{D^{q}} \Lambda_{D^{q}}^{\dagger}\right)_{i l}-\frac{6}{5} g_{1}^{2}\left(h_{E} Y_{E}^{\dagger}+h_{E^{q}} \Lambda_{E^{q}}^{\dagger}\right)_{i l} \\
& +2\left(h_{E} Y_{E}^{\dagger} E+E h_{E} Y_{E}^{\dagger}\right)_{i l}+\left(Y_{E}^{\dagger}\right)_{q l}\left(h_{E}\right)_{i n} \operatorname{Tr}\left(\Lambda_{E^{n}}^{\dagger} \Lambda_{E^{q}}\right) \\
& +2\left(\Lambda_{E^{n}} \Lambda_{E q}^{\dagger}\right)_{i l}\left(Y_{E}^{\dagger} h_{E}\right)_{n q}+\left(h_{E} Y_{E}^{\dagger}\right)_{i l} \operatorname{Tr}(E+3 D) \\
& +E_{i l} \operatorname{Tr}\left(h_{E} Y_{E}^{\dagger}\right)+\left(h_{E}\right)_{i q}\left(3 \Lambda_{E q}^{\dagger} \Lambda_{D p} Y_{D}^{*}+\Lambda_{E q}^{\dagger} \Lambda_{E} p Y_{D}^{*}\right)_{l p} \\
& -\left(Y_{E}^{\dagger}\right)_{q l}\left(\Lambda_{E^{q}} \Lambda_{E^{p}}^{*} h_{E}\right)_{i p}+\left(\Lambda_{E^{q}}^{\dagger} h_{E} Y_{E}^{\dagger} \Lambda_{E^{q}}\right)_{l i}+3\left(\Lambda_{D^{q}}^{*} h_{U} Y_{U}^{\dagger} \Lambda_{D^{q}}^{t}\right)_{l i} \\
& +3(E)_{i l} \operatorname{Tr}\left(h_{D} Y_{D}^{\dagger}\right)-3\left(Y_{E}^{\dagger}\right)_{q l}\left(\Lambda_{E^{q}} \Lambda_{D^{p}}^{*} h_{D}\right)_{i p}+6\left(\Lambda_{D^{n}} \Lambda_{D^{q}}^{\dagger}\right)_{i l}\left(Y_{D}^{\dagger} h_{D}\right)_{n q} \\
& +3\left(\Lambda_{D^{q}}^{*} h_{D} Y_{D^{\dagger}}^{\dagger} \Lambda_{D^{q}}^{\dagger}\right)_{l i}+\left(Y_{E}^{\dagger}\right)_{q l}\left(Y_{E}\right)_{i n} \operatorname{Tr}\left(\Lambda_{E^{n}}^{\dagger} h_{E^{q}}\right)+2\left(h_{E^{n}} \Lambda_{E^{q}}^{\dagger}\right)_{i l}(\tilde{E})_{n q} \\
& +\left(h_{E^{n}} \Lambda_{E^{q}}^{\dagger}\right)_{i l} \operatorname{Tr}\left(\Lambda_{E^{n}}^{\dagger} \Lambda_{E^{q}}\right)+\left(\Lambda_{E^{n}} \Lambda_{E^{q}}^{\dagger}\right)_{i l} \operatorname{Tr}\left(\Lambda_{E^{n}}^{\dagger} h_{E^{q}}\right)+\left(Y_{E}\right)_{i q}\left(\Lambda_{E^{q}}^{\dagger} h_{E^{p}} Y_{E}^{*}\right)_{l p} \\
& -3\left(Y_{E}^{\dagger}\right)_{q l}\left(h_{E^{q}} \Lambda_{D^{p}}^{*} Y_{D}\right)_{i p}-\left(Y_{E}^{\dagger}\right)_{q l}\left(h_{E^{q}} \Lambda_{E^{p}}^{*} Y_{E}\right)_{i p}+\left(\Lambda_{E^{q}}^{\dagger} E h_{E^{q}}\right. \\
& \left.+3 \Lambda_{E^{q}}^{\dagger} \Lambda_{D^{p}} \Lambda_{D^{p}}^{\dagger} h_{E^{q}}\right)_{l i}+\left(\Lambda_{E^{q}}^{\dagger} h_{E^{p}} \Lambda_{E^{p}}^{\dagger} \Lambda_{E^{q}}+\Lambda_{E^{q}}^{\dagger} \Lambda_{E^{p}} \Lambda_{E^{p}}^{\dagger} h_{E^{q}}\right)_{l i} \\
& +6\left(\Lambda_{D^{n}} \Lambda_{D^{q}}^{\dagger}\right)_{i l}\left(h_{U^{m}} \Lambda_{U^{m}}^{\dagger}\right)_{q n}+3\left(Y_{E}\right)_{i q}\left(\Lambda_{E^{q}}^{\dagger} h_{D^{p}} Y_{D}^{*}\right)_{l p}+3\left(\Lambda_{E^{q}}^{\dagger} h_{D^{p}} \Lambda_{D^{p}}^{\dagger} \Lambda_{E^{q}}\right)_{l i} \\
& +6\left(h_{D^{n}} \Lambda_{D^{q}}^{\dagger}\right)_{i l}\left[(\tilde{D})_{n q}+\operatorname{Tr}\left(\Lambda_{D^{n}}^{\dagger} \Lambda_{D^{q}}\right)+\left(\Lambda_{U^{m}} \Lambda_{U^{m}}^{\dagger}\right)_{q n}\right] \\
& +6\left(\Lambda_{D^{n}} \Lambda_{D^{q}}^{\dagger}\right)_{i l} \operatorname{Tr}\left(\Lambda_{D^{n}}^{\dagger} h_{D^{q}}\right)+3\left(\Lambda_{D^{q}}^{*} D h_{D^{q}}^{t}+\Lambda_{D^{q}}^{*} T h_{D^{q}}^{t}\right)_{l i} \\
& \left.+3\left(\Lambda_{D^{q}} \Lambda_{D^{p}}^{\dagger} h_{D^{p}} \Lambda_{D^{q}}^{\dagger}+h_{D^{q}} \Lambda_{D^{p}}^{\dagger} \Lambda_{D^{p}} \Lambda_{D^{q}}^{\dagger}\right)_{i l}\right\} \\
& +\left(h_{E^{k}}\right)_{i j}\left\{\frac{234}{25} g_{1}^{4}+\frac{15}{2} g_{2}^{4}+\frac{207}{50} g_{1}^{4}+\frac{9}{5} g_{1}^{2} g_{2}^{2}\right\} \\
& \left.-\left(\Lambda_{E^{k}}\right)_{i j}\left\{30 M_{2} g_{2}^{4}+\frac{18}{5}\left(M_{1}+M_{2}\right) g_{2}^{2} g_{1}^{2}+\frac{414}{25} M_{1} g_{1}^{4}+4\left(\frac{936}{25}\right) g_{1}^{4}\right)\right\} \\
& \left(16 \pi^{2}\right)^{2} \beta_{\left(h_{D^{k}}\right)_{i j}}^{(2)}=\left(h_{D^{l}}\right)_{i j}\left\{\left(\frac{16}{3} g_{3}^{2}+\frac{16}{15} g_{1}^{2}\right)\left(\Lambda_{U^{q}} \Lambda_{U^{q}}^{\dagger}\right)_{k l}-2(D)_{l k} \operatorname{Tr}(E+3 D)\right. \\
& +\left(6 g_{2}^{2}+\frac{2}{5} g_{1}^{2}\right)\left[D_{k l}+\operatorname{Tr}\left(\Lambda_{D^{k}} \Lambda_{D^{l}}^{\dagger}\right)\right]-2\left(Y_{D}^{\dagger} D Y_{D}\right)_{l k}-2\left(Y_{D}^{\dagger} T Y_{D}\right)_{l k} \\
& -2\left(Y_{D}^{t} \Lambda_{D^{p}}^{\dagger} \Lambda_{D^{p}} Y_{D}^{*}\right)_{k l}-2 \operatorname{Tr}\left(\Lambda_{D^{k}}^{t} \Lambda_{D^{l}}^{*}[D+T]\right. \\
& \left.+\Lambda_{D^{l}}^{\dagger} \Lambda_{D^{k}} \Lambda_{D^{q}}^{\dagger} \Lambda_{D^{q}}\right)-\operatorname{Tr}\left(6 \Lambda_{D^{q}} \Lambda_{D^{q}}^{\dagger} \Lambda_{D^{k}} \Lambda_{D^{l}}^{\dagger}+2 \Lambda_{E^{q}} \Lambda_{E^{q}}^{\dagger} \Lambda_{D^{k}} \Lambda_{D^{l}}^{\dagger}\right. \\
& \left.+2 E \Lambda_{D^{k}} \Lambda_{D^{l}}^{\dagger}\right)-4\left(\Lambda_{U^{m}} \tilde{D} \Lambda_{U^{m}}^{\dagger}\right)_{k l}-4\left(\Lambda_{U^{m}}^{\dagger} \Lambda_{U^{p}} \Lambda_{U^{p}}^{\dagger} \Lambda_{U^{m}}\right)_{l k} \\
& +4\left(\Lambda_{U^{m}}^{\dagger}\right)_{l q}\left(\Lambda_{U^{m}}\right)_{k n} \operatorname{Tr}\left(\Lambda_{D^{n}} \Lambda_{D^{q}}^{\dagger}\right)-\left(6 Y_{D^{\dagger}}^{\dagger} \Lambda_{D^{k}}^{t} \Lambda_{D^{p}}^{*} Y_{D}\right)_{l p}
\end{aligned}
$$




$$
\begin{aligned}
& -2\left(Y_{D}^{\dagger} \Lambda_{D^{k}}^{t} \Lambda_{E^{p}}^{*} Y_{E}\right)_{l p}-\left(6 Y_{D}^{t} \Lambda_{D^{l}}^{\dagger} \Lambda_{D^{p}} Y_{D}^{*}+2 Y_{D}^{t} \Lambda_{D^{l}}^{\dagger} \Lambda_{E^{p}} Y_{E}^{*}\right)_{k p} \\
& \left.-2\left(\Lambda_{U^{q}}^{\dagger} \Lambda_{U^{n}}\right)_{l k}\left[\operatorname{Tr}\left(\Lambda_{U^{n}}^{\dagger} \Lambda_{U^{q}}\right)+2(\tilde{T})_{n q}\right]\right\} \\
& +\left(h_{D^{k}}\right)_{i l}\left\{\frac{2}{5} g_{1}^{2}\left[(D+2 T)_{j l}+\left(\Lambda_{D^{q}}^{\dagger} \Lambda_{D^{q}}\right)_{l j}\right]-\left(\Lambda_{D^{m}}^{\dagger} E \Lambda_{D^{m}}\right)_{l j}\right. \\
& -2\left(D^{2}\right)_{j l}-(D)_{j l} \operatorname{Tr}(E+3 D)-2\left(T^{2}\right)_{j l}-3(T)_{j l} \operatorname{Tr}(T) \\
& -2\left(\Lambda_{D^{n}}^{\dagger} \Lambda_{D^{m}}\right)_{l j}\left[(\tilde{D})_{m n}+\left(\Lambda_{U^{q}} \Lambda_{U^{q}}^{\dagger}\right)_{n m}+\operatorname{Tr}\left(\Lambda_{D^{m}}^{\dagger} \Lambda_{D^{n}}\right)\right] \\
& -3\left(\Lambda_{D^{m}}^{\dagger} \Lambda_{D^{q}} \Lambda_{D^{q}}^{\dagger} \Lambda_{D^{m}}\right)_{l j}-\left(\Lambda_{D^{m}}^{\dagger} \Lambda_{E^{q}} \Lambda_{E^{q}}^{\dagger} \Lambda_{D^{m}}\right)_{l j}-\left(Y_{D}\right)_{j n}\left(3 \Lambda_{D^{n}}^{\dagger} \Lambda_{D^{m}} Y_{D}^{*}\right. \\
& \left.+\Lambda_{D^{n}}^{\dagger} \Lambda_{E^{m}} Y_{E}^{*}\right)_{l m}-2\left(Y_{D} \Lambda_{U^{q}}^{\dagger} \Lambda_{U^{q}} Y_{D}^{\dagger}\right)_{j l}-2\left(Y_{D}\right)_{j m}\left(Y_{D}^{\dagger}\right)_{n l} \operatorname{Tr}\left(\Lambda_{D^{m}}^{\dagger} \Lambda_{D^{n}}\right) \\
& \left.-\left(Y_{D}^{*}\right)_{l n}\left(3 \Lambda_{D^{n}}^{t} \Lambda_{D^{m}}^{*} Y_{D}+\Lambda_{D^{n}}^{t} \Lambda_{E^{m}}^{*} Y_{E}\right)_{j m}-\left(Y_{U}\right)_{j n}\left(Y_{U}^{\dagger}\right)_{q l} \operatorname{Tr}\left(\Lambda_{U^{n}}^{\dagger} \Lambda_{U^{q}}\right)\right\} \\
& +\left(h_{D^{k}}\right)_{l j}\left\{\left(16 g_{3}^{2}-\frac{2}{5} g_{1}^{2}\right)\left(\Lambda_{D^{q}} \Lambda_{D^{q}}^{\dagger}\right)_{i l}+\frac{6}{5} g_{1}^{2}\left(E+\Lambda_{E^{q}} \Lambda_{E^{q}}^{\dagger}\right)_{i l}\right. \\
& -2\left(E^{2}\right)_{i l}-\left(Y_{E}^{\dagger}\right)_{q l}\left(Y_{E}\right)_{i n} \operatorname{Tr}\left(\Lambda_{E^{n}}^{\dagger} \Lambda_{E^{q}}\right)-2\left(\Lambda_{E^{n}} \Lambda_{E^{q}}^{\dagger}\right)_{i l}(\tilde{E})_{n q} \\
& -\left(\Lambda_{E^{n}} \Lambda_{E^{q}}^{\dagger}\right)_{i l} \operatorname{Tr}\left(\Lambda_{E^{n}}^{\dagger} \Lambda_{E^{q}}\right)-(E)_{i l} \operatorname{Tr}(E+3 D) \\
& -\left(Y_{E}\right)_{i q}\left(3 \Lambda_{E^{q}}^{\dagger} \Lambda_{D^{p}} Y_{D}^{*}+\Lambda_{E^{q}}^{\dagger} \Lambda_{E^{p}} Y_{E}^{*}\right)_{l p}+\left(Y_{E}^{*}\right)_{l q}\left(3 \Lambda_{E^{q}} \Lambda_{D^{p}}^{*} Y_{D}\right. \\
& \left.+\Lambda_{E^{q}} \Lambda_{E^{p}}^{*} Y_{E}\right)_{i p}-\left(\Lambda_{E^{q}}^{\dagger} E \Lambda_{E^{q}}+3 \Lambda_{E^{q}}^{\dagger} \Lambda_{D^{p}} \Lambda_{D^{p}}^{\dagger} \Lambda_{E^{q}}+\Lambda_{E^{q}}^{\dagger} \Lambda_{E^{p}} \Lambda_{E^{p}}^{\dagger} \Lambda_{E^{q}}\right)_{l i} \\
& -6\left(\Lambda_{D^{n}} \Lambda_{D^{q}}^{\dagger}\right)_{i l}\left[(\tilde{D})_{n q}+\operatorname{Tr}\left(\Lambda_{D^{n}}^{\dagger} \Lambda_{D^{q}}\right)+\left(\Lambda_{U^{m}} \Lambda_{U^{m}}^{\dagger}\right)_{q n}\right] \\
& \left.-3\left(\Lambda_{D^{q}}^{*} D \Lambda_{D^{q}}^{t}+\Lambda_{D^{q}}^{*} T \Lambda_{D^{q}}^{t}\right)_{l i}-3\left(\Lambda_{D^{q}} \Lambda_{D^{p}}^{\dagger} \Lambda_{D^{p}} \Lambda_{D^{q}}^{\dagger}\right)_{i l}\right\} \\
& -\left(h_{D}\right)_{j k}\left\{\left(\frac{2}{5} g_{1}^{2}-16 g_{3}^{2}\right)\left(\Lambda_{D q} Y_{D}^{*}\right)_{i q}-\frac{6}{5} g_{1}^{2}\left(\Lambda_{E q} Y_{E}^{*}\right)_{i q}\right. \\
& -\left(3 \Lambda_{E^{n}}^{t} \Lambda_{D^{p}}^{*} \Lambda_{D^{p}}^{t} Y_{E}^{*}+\Lambda_{E^{n}}^{t} \Lambda_{E^{p}}^{*} \Lambda_{E^{p}}^{t} Y_{E}^{*}+3 \Lambda_{E^{n}}^{t} E^{t} Y_{E}^{*}\right)_{i n} \\
& -\left(\Lambda_{E^{q}}^{t} Y_{E}^{*}\right)_{i l} \operatorname{Tr}\left(\Lambda_{E^{l}}^{t} \Lambda_{E^{q}}^{*}\right)-3\left(E \Lambda_{D^{m}} Y_{D}^{*}-E \Lambda_{E^{m}} Y_{E}^{*}\right)_{i m} \\
& +9\left(\Lambda_{D^{q}} D^{t} Y_{D}^{*}\right)_{i q}-6\left(\Lambda_{D^{q}} Y_{D}^{*} \Lambda_{U^{m}}^{t} \Lambda_{U^{m}}^{*}\right)_{i q}+6\left(\Lambda_{D^{q}} Y_{D}^{*}\right)_{i l} \operatorname{Tr}\left(\Lambda_{D^{l}}^{*} \Lambda_{D^{q}}^{t}\right) \\
& \left.+3\left(\Lambda_{D^{n}} Y_{U}^{*} Y_{U}^{t} Y_{D}^{*}+\Lambda_{D^{n}} \Lambda_{D^{m}}^{\dagger} \Lambda_{D^{m}} Y_{D}^{*}\right)_{i n}\right\} \\
& -2\left(\Lambda_{D^{l}}\right)_{i j}\left\{\left(\frac{16}{3} M_{3} g_{3}^{2}+\frac{16}{15} M_{1} g_{1}^{2}\right)\left(\Lambda_{U^{q}} \Lambda_{U^{q}}^{\dagger}\right)_{k l}\right. \\
& +\left(6 M_{2} g_{2}^{2}+\frac{2}{5} M_{1} g_{1}^{2}\right)\left[(D)_{k l}+\operatorname{Tr}\left(\Lambda_{D^{k}} \Lambda_{D^{l}}^{\dagger}\right)\right]+2\left(h_{D}^{t} \Lambda_{D^{p}}^{\dagger} \Lambda_{D^{p}} Y_{D}^{*}\right)_{k l} \\
& -\left(\frac{16}{3} g_{3}^{2}+\frac{16}{15} g_{1}^{2}\right)\left(h_{U^{q}} \Lambda_{U^{q}}^{\dagger}\right)_{k l}-\left(6 g_{2}^{2}+\frac{2}{5} g_{1}^{2}\right)\left[\left(h_{D} Y_{D}^{\dagger}\right)_{k l}+\operatorname{Tr}\left(h_{D^{k}} \Lambda_{D^{l}}^{\dagger}\right)\right] \\
& +2(\tilde{D})_{l k} \operatorname{Tr}\left(Y_{E}^{\dagger} h_{E}\right)+2 \operatorname{Tr}\left(h_{E} Y_{E}^{\dagger} \Lambda_{D^{k}} \Lambda_{D^{l}}^{\dagger}\right)+2\left(Y_{D^{\dagger}}^{\dagger} \Lambda_{D^{k}}^{t} \Lambda_{E^{p}}^{*} h_{E}\right)_{l p} \\
& +2\left(Y_{D}^{\dagger} h_{U} Y_{U}^{\dagger} Y_{D}\right)_{l k}+2 \operatorname{Tr}\left(\Lambda_{D^{k}}^{t} \Lambda_{D^{l}}^{*} h_{U} Y_{U}^{\dagger}\right)+4\left(\Lambda_{U^{q}}^{\dagger} \Lambda_{U^{n}}\right)_{l k}\left(Y_{U}^{\dagger} h_{U}\right)_{n q} \\
& +2\left(Y_{D}^{\dagger} h_{D} \tilde{D}\right)_{l k}+2\left(Y_{D}^{\dagger} D h_{D}\right)_{l k}+2\left(Y_{D}^{\dagger} T h_{D}\right)_{l k} \\
& +2\left(Y_{D}^{\dagger} h_{D}\right)_{l k} \operatorname{Tr}(E+3 D)+6 \tilde{D}_{l k} \operatorname{Tr}\left(Y_{D}^{\dagger} h_{D}\right) \\
& +2 \operatorname{Tr}\left(\Lambda_{D^{k}}^{t} \Lambda_{D^{l}}^{*} h_{D} Y_{D}^{\dagger}\right)+4\left(\Lambda_{U^{m}} Y_{D}^{\dagger} h_{D} \Lambda_{U^{m}}^{\dagger}\right)_{k l}+6\left(Y_{D^{\dagger}}^{\dagger} \Lambda_{D^{k}}^{t} \Lambda_{D^{p}}^{*} h_{D}\right)_{l p} \\
& +6\left(h_{D}^{t} \Lambda_{D^{l}}^{\dagger} \Lambda_{D^{p}} Y_{D}^{*}+2 h_{D}^{t} \Lambda_{D^{l}}^{\dagger} \Lambda_{E^{p}} Y_{E}^{*}\right)_{k p}+\operatorname{Tr}\left(2 h_{E^{q}} \Lambda_{E^{q}}^{\dagger} \Lambda_{D^{k}} \Lambda_{D^{l}}^{\dagger}\right) \\
& +2\left(Y_{D}^{t} \Lambda_{D^{l}}^{\dagger} h_{E^{p}} Y_{E}^{*}\right)_{k p}+4\left(h_{U^{m}} \tilde{D} \Lambda_{U^{m}}^{\dagger}\right)_{k l}+4\left(\Lambda_{U^{m}}^{\dagger} h_{U^{p}} \Lambda_{U^{p}}^{\dagger} \Lambda_{U^{m}}\right)_{l k} \\
& +4\left(\Lambda_{U^{m}}^{\dagger} \Lambda_{U^{p}} \Lambda_{U^{p}}^{\dagger} h_{U^{m}}\right)_{l k}-4\left(\Lambda_{U^{m}}^{\dagger}\right)_{l q}\left(h_{U^{m}}\right)_{k n} \operatorname{Tr}\left(\Lambda_{D^{n}} \Lambda_{D^{q}}^{\dagger}\right)+2\left(Y_{D}^{t} \Lambda_{D^{p}}^{\dagger} h_{D^{p}} Y_{D}^{*}\right)_{k l} \\
& +2\left(\Lambda_{U^{q}}^{\dagger} h_{U^{n}}\right)_{l k}\left[\operatorname{Tr}\left(\Lambda_{U^{n}}^{\dagger} \Lambda_{U^{q}}\right)+2(\tilde{T})_{n q}\right]+2\left(\Lambda_{U^{q}}^{\dagger} \Lambda_{U^{n}}\right)_{l k} \operatorname{Tr}\left(\Lambda_{U^{n}}^{\dagger} h_{U^{q}}\right) \\
& +2 \operatorname{Tr}\left(h_{D^{k}}^{t} \Lambda_{D^{l}}^{*}(D+T)+\Lambda_{D^{l}}^{\dagger} h_{D^{k}} \Lambda_{D^{q}}^{\dagger} \Lambda_{D^{q}}+\Lambda_{D^{l}}^{\dagger} \Lambda_{D^{k}} \Lambda_{D^{q}}^{\dagger} h_{D^{q}}\right) \\
& +\operatorname{Tr}\left(6 h_{D^{q}} \Lambda_{D^{q}}^{\dagger} \Lambda_{D^{k}} \Lambda_{D^{l}}^{\dagger}+6 \Lambda_{D^{q}} \Lambda_{D^{q}}^{\dagger} h_{D^{k}} \Lambda_{D^{l}}^{\dagger}+2 \Lambda_{E^{q}} \Lambda_{E^{q}}^{\dagger} h_{D^{k}} \Lambda_{D^{l}}^{\dagger}+2 E h_{D^{k}} \Lambda_{D^{l}}^{\dagger}\right) \\
& -4\left(\Lambda_{U^{m}}^{\dagger}\right)_{l q}\left(\Lambda_{U^{m}}\right)_{k n} \operatorname{Tr}\left(h_{D^{n}} \Lambda_{D^{q}}^{\dagger}\right)+\left(6 Y_{D^{\dagger}}^{\dagger} h_{D^{k}}^{t} \Lambda_{D^{p}}^{*} Y_{D}\right)_{l p}
\end{aligned}
$$




$$
\begin{aligned}
& \left.+2\left(Y_{D}^{\dagger} h_{D^{k}}^{t} \Lambda_{E^{p}}^{*} Y_{E}\right)_{l p}+\left(6 Y_{D}^{t} \Lambda_{D^{l}}^{\dagger} h_{D^{p}} Y_{D}^{*}\right)_{k p}\right\} \\
& -2\left(\Lambda_{D^{k}}\right)_{i l}\left\{\frac{2}{5} M_{1} g_{1}^{2}\left[(D+2 T)_{j l}+\left(\Lambda_{D^{q}}^{\dagger} \Lambda_{D^{q}}\right)_{l j}\right]\right. \\
& -\frac{2}{5} g_{1}^{2}\left[\left(h_{D} Y_{D}^{\dagger}+2 h_{U} Y_{U}^{\dagger}\right)_{j l}+\left(\Lambda_{D^{q}}^{\dagger} h_{D^{q}}\right)_{l j}\right]+3\left(h_{U} Y_{U}^{\dagger}\right)_{j l} \operatorname{Tr}(T) \\
& +(D)_{j l} \operatorname{Tr}\left(Y_{E}^{\dagger} h_{E}\right)+\left(\Lambda_{D^{m}}^{\dagger} h_{E} Y_{E}^{\dagger} \Lambda_{D^{m}}\right)_{l j}+\left(Y_{D}^{*}\right)_{l n}\left(3 \Lambda_{D^{n}}^{t} \Lambda_{E^{m}}^{*} h_{E}\right)_{j m} \\
& +2\left(h_{U} Y_{U}^{\dagger} T\right)_{j l}+2\left(T h_{U} Y_{U}^{\dagger}\right)_{j l}+\left(Y_{D}\right)_{j n}\left(\Lambda_{D^{n}}^{\dagger} h_{E^{m}} Y_{E}^{*}\right)_{l m} \\
& +3(T)_{j l} \operatorname{Tr}\left(Y_{U}^{\dagger} h_{U}\right)+\left(h_{U}\right)_{j n}\left(Y_{U}^{\dagger}\right)_{q l} \operatorname{Tr}\left(\Lambda_{U^{n}}^{\dagger} \Lambda_{U^{q}}\right)+2\left(h_{D} Y_{D}^{\dagger} D\right. \\
& \left.+D h_{D} Y_{D}^{\dagger}\right)_{j l}+\left(h_{D} Y_{D}^{\dagger}\right)_{j l} \operatorname{Tr}(E+3 D)+\left(Y_{U}\right)_{j n}\left(Y_{U}^{\dagger}\right)_{q l} \operatorname{Tr}\left(\Lambda_{U^{n}}^{\dagger} h_{U^{q}}\right) \\
& +(D)_{j l} \operatorname{Tr}\left(3 Y_{D^{\dagger}}^{\dagger} h_{D}\right)+2\left(\Lambda_{D^{n}}^{\dagger} \Lambda_{D^{m}}\right)_{l j}\left(Y_{D}^{\dagger} h_{D}\right)_{m n}+\left(h_{D}\right)_{j n}\left(3 \Lambda_{D^{n}}^{\dagger} \Lambda_{D^{m}} Y_{D}^{*}\right. \\
& \left.+\Lambda_{D^{n}}^{\dagger} \Lambda_{E^{m}} Y_{E}^{*}\right)_{l m}+2\left(h_{D} \Lambda_{U^{q}}^{\dagger} \Lambda_{U^{q}} Y_{D}^{\dagger}\right)_{j l}+2\left(h_{D}\right)_{j m}\left(Y_{D}^{\dagger}\right)_{n l} \operatorname{Tr}\left(\Lambda_{D^{m}}^{\dagger} \Lambda_{D^{n}}\right) \\
& +3\left(Y_{D}^{*}\right)_{l n}\left(\Lambda_{D^{n}}^{t} \Lambda_{D^{m}}^{*} h_{D}\right)_{j m}+\left(\Lambda_{D^{m}}^{\dagger} h_{E^{q}} \Lambda_{E^{q}}^{\dagger} \Lambda_{D^{m}}\right)_{l j} \\
& +2\left(\Lambda_{D^{n}}^{\dagger} \Lambda_{D^{n}}\right)_{l j}\left(h_{U^{q}} \Lambda_{U^{q}}^{\dagger}\right)_{n m}+2\left(Y_{D} \Lambda_{U^{q}}^{\dagger} h_{U^{q}} Y_{D}^{\dagger}\right)_{j l} \\
& +2\left(\Lambda_{D^{n}}^{\dagger} h_{D^{m}}\right)_{l j}\left[(\tilde{D})_{m n}+\left(\Lambda_{U^{q}} \Lambda_{U^{q}}^{\dagger}\right)_{n m}+\operatorname{Tr}\left(\Lambda_{D^{m}}^{\dagger} \Lambda_{D^{n}}\right)\right] \\
& +2\left(\Lambda_{D^{n}}^{\dagger} \Lambda_{D^{m}}\right)_{l j} \operatorname{Tr}\left(\Lambda_{D^{m}}^{\dagger} h_{D^{n}}\right)+\left(\Lambda_{D^{m}}^{\dagger} E h_{D^{m}}\right)_{l j}+3\left(\Lambda_{D^{m}}^{\dagger} h_{D^{q}} \Lambda_{D^{q}}^{\dagger} \Lambda_{D^{m}}\right. \\
& \left.+\Lambda_{D^{m}}^{\dagger} \Lambda_{D^{q}} \Lambda_{D^{q}}^{\dagger} h_{D^{m}}\right)_{l j}+\left(\Lambda_{D^{m}}^{\dagger} \Lambda_{E^{q}} \Lambda_{E^{q}}^{\dagger} h_{D^{m}}\right)_{l j}+3\left(Y_{D}\right)_{j n}\left(\Lambda_{D^{n}}^{\dagger} h_{D^{m}} Y_{D}^{*}\right)_{l m} \\
& \left.+2\left(Y_{D}\right)_{j m}\left(Y_{D}^{\dagger}\right)_{n l} \operatorname{Tr}\left(\Lambda_{D^{m}}^{\dagger} h_{D^{n}}\right)+\left(Y_{D}^{*}\right)_{l n}\left(3 h_{D^{n}}^{t} \Lambda_{D^{m}}^{*} Y_{D}+h_{D^{n}}^{t} \Lambda_{E^{m}}^{*} Y_{E}\right)_{j m}\right\} \\
& -2\left(\Lambda_{D^{k}}\right)_{l j}\left\{\left(16 M_{3} g_{3}^{2}-\frac{2}{5} M_{1} g_{1}^{2}\right)\left(\Lambda_{D^{q}} \Lambda_{D^{q}}^{\dagger}\right)_{i l}+\frac{6}{5} M_{1} g_{1}^{2}\left(E+\Lambda_{E^{q}} \Lambda_{E^{q}}^{\dagger}\right)_{i l}\right. \\
& -\left(16 g_{3}^{2}-\frac{2}{5} g_{1}^{2}\right)\left(h_{D^{q}} \Lambda_{D^{q}}^{\dagger}\right)_{i l}-\frac{6}{5} g_{1}^{2}\left(h_{E} Y_{E}^{\dagger}+h_{E^{q}} \Lambda_{E^{q}}^{\dagger}\right)_{i l} \\
& +2\left(h_{E} Y_{E}^{\dagger} E+E h_{E} Y_{E}^{\dagger}\right)_{i l}+\left(Y_{E}^{\dagger}\right)_{q l}\left(h_{E}\right)_{i n} \operatorname{Tr}\left(\Lambda_{E^{n}}^{\dagger} \Lambda_{E^{q}}\right) \\
& +\left(h_{E} Y_{E}^{\dagger}\right)_{i l} \operatorname{Tr}(E+3 D)+(E)_{i l} \operatorname{Tr}\left(h_{E} Y_{E}^{\dagger}+3 h_{D} Y_{D}^{\dagger}\right) \\
& +\left(h_{E}\right)_{i q}\left(3 \Lambda_{E q}^{\dagger} \Lambda_{D^{p}} Y_{D}^{*}+\Lambda_{E^{q}}^{\dagger} \Lambda_{E^{p}} Y_{E}^{*}\right)_{l p}-\left(Y_{E}^{*}\right)_{l q}\left(\Lambda_{E^{q}} \Lambda_{E^{p}}^{*} h_{E}\right)_{i p} \\
& +\left(\Lambda_{E^{q}}^{\dagger} h_{E} Y_{E}^{\dagger} \Lambda_{E^{q}}\right)_{l i}+3\left(\Lambda_{D^{q}}^{*} h_{U} Y_{U}^{\dagger} \Lambda_{D^{q}}^{t}\right)_{l i}+2\left(\Lambda_{E^{n}} \Lambda_{E^{q}}^{\dagger}\right)_{i l}\left(Y_{E}^{\dagger} h_{E}\right)_{n q} \\
& -3\left(Y_{E}^{*}\right)_{l q}\left(\Lambda_{E^{q}} \Lambda_{D^{p}}^{*} h_{D}\right)_{i p}+6\left(\Lambda_{D^{n}} \Lambda_{D^{q}}^{\dagger}\right)_{i l}\left(Y_{D}^{\dagger} h_{D}\right)_{n q}+3\left(\Lambda_{D^{q}}^{*} h_{D} Y_{D}^{\dagger} \Lambda_{D^{q}}^{t}\right)_{l i} \\
& +\left(Y_{E}^{\dagger}\right)_{q l}\left(Y_{E}\right)_{i n} \operatorname{Tr}\left(\Lambda_{E^{n}}^{\dagger} h_{E^{q}}\right)+2\left(h_{E^{n}} \Lambda_{E^{q}}^{\dagger}\right)_{i l}(\tilde{E})_{n q} \\
& +\left(\Lambda_{E^{n}} \Lambda_{E^{q}}^{\dagger}\right)_{i l} \operatorname{Tr}\left(\Lambda_{E^{n}}^{\dagger} h_{E^{q}}\right)+\left(Y_{E}\right)_{i q}\left(\Lambda_{E^{q}}^{\dagger} h_{E^{p}} Y_{E}^{*}\right)_{l p}-\left(Y_{E}^{*}\right)_{l q}\left(3 h_{E^{q}} \Lambda_{D^{p}}^{*} Y_{D}\right. \\
& \left.+h_{E^{q}} \Lambda_{E p}^{*} Y_{E}\right)_{i p}+\left(\Lambda_{E q}^{\dagger} E h_{E^{q}}+3 \Lambda_{E^{q}}^{\dagger} \Lambda_{D^{p}} \Lambda_{D^{p}}^{\dagger} h_{E^{q}}\right. \\
& \left.+\Lambda_{E^{q}}^{\dagger} h_{E^{p}} \Lambda_{E^{p}}^{\dagger} \Lambda_{E^{q}}+\Lambda_{E^{q}}^{\dagger} \Lambda_{E^{p}} \Lambda_{E^{p}}^{\dagger} h_{E^{q}}\right)_{l i}+6\left(\Lambda_{D^{n}} \Lambda_{D^{q}}^{\dagger}\right)_{i l}\left(h_{U^{m}} \Lambda_{U^{m}}^{\dagger}\right)_{q n} \\
& +3\left(Y_{E}\right)_{i q}\left(\Lambda_{E^{q}}^{\dagger} h_{D^{p}} Y_{D}^{*}\right)_{l p}+3\left(\Lambda_{E^{q}}^{\dagger} h_{D^{p}} \Lambda_{D^{p}}^{\dagger} \Lambda_{E^{q}}\right)_{l i}+6\left(h_{D^{n}} \Lambda_{D^{q}}^{\dagger}\right)_{i l}\left[(\tilde{D})_{n q}\right. \\
& \left.+\operatorname{Tr}\left(\Lambda_{D^{n}}^{\dagger} \Lambda_{D^{q}}\right)+\left(\Lambda_{U^{m}} \Lambda_{U^{m}}^{\dagger}\right)_{q n}\right]+6\left(\Lambda_{D^{n}} \Lambda_{D^{q}}^{\dagger}\right)_{i l} \operatorname{Tr}\left(\Lambda_{D^{n}}^{\dagger} h_{D^{q}}\right) \\
& +3\left(\Lambda_{D^{q}}^{*} D h_{D^{q}}^{t}+\Lambda_{D^{q}}^{*} T h_{D^{q}}^{t}\right)_{l i}+\left(h_{E^{n}} \Lambda_{E^{q}}^{\dagger}\right)_{i l} \operatorname{Tr}\left(\Lambda_{E^{n}}^{\dagger} \Lambda_{E^{q}}\right) \\
& \left.+3\left(\Lambda_{D^{q}} \Lambda_{D^{p}}^{\dagger} h_{D^{p}} \Lambda_{D^{q}}^{\dagger}+h_{D^{q}} \Lambda_{D^{p}}^{\dagger} \Lambda_{D^{p}} \Lambda_{D^{q}}^{\dagger}\right)_{i l}\right\} \\
& +2\left(Y_{D}\right)_{j k}\left\{\left(\frac{2}{5} M_{1} g_{1}^{2}-16 M_{3} g_{3}^{2}\right)\left(\Lambda_{D^{q}} Y_{D}^{*}\right)_{i q}-\frac{6}{5} M_{1} g_{1}^{2}\left(\Lambda_{E^{q}} Y_{E}^{*}\right)_{i q}\right. \\
& -\left(\frac{2}{5} g_{1}^{2}-16 g_{3}^{2}\right)\left(h_{D^{q}} Y_{D}^{*}\right)_{i q}+\frac{6}{5} g_{1}^{2}\left(h_{E^{q}} Y_{E}^{*}\right)_{i q}+\left(3 \Lambda_{E^{n}}^{t} Y_{E}^{*} h_{E}^{t} Y_{E}^{*}\right)_{i n} \\
& +3\left(h_{E} Y_{E}^{\dagger} \Lambda_{D^{m}} Y_{D}^{*}-h_{E} Y_{E}^{\dagger} \Lambda_{E m} Y_{E}^{*}\right)_{i m}-3\left(\Lambda_{D^{n}} Y_{U}^{*} h_{U}^{t} Y_{D}^{*}\right)_{i n} \\
& -9\left(\Lambda_{D^{q}} Y_{D}^{*} h_{D}^{t} Y_{D}^{*}\right)_{i q}+\left(3 h_{E^{n}}^{t} \Lambda_{D^{p}}^{*} \Lambda_{D^{p}}^{t} Y_{E}^{*}+h_{E^{n}}^{t} \Lambda_{E^{p}}^{*} \Lambda_{E^{p}}^{t} Y_{E}^{*}\right. \\
& \left.+\Lambda_{E^{n}}^{t} \Lambda_{E^{p}}^{*} h_{E^{p}}^{t} Y_{E}^{*}+3 h_{E^{n}}^{t} E^{t} Y_{E}^{*}\right)_{i n}+\left(h_{E^{q}}^{t} Y_{E}^{*}\right)_{i l} \operatorname{Tr}\left(\Lambda_{E^{l}}^{t} \Lambda_{E^{q}}^{*}\right)
\end{aligned}
$$




$$
\begin{aligned}
& +\left(\Lambda_{E^{q}}^{t} Y_{E}^{*}\right)_{i l} \operatorname{Tr}\left(h_{E^{l}}^{t} \Lambda_{E^{q}}^{*}\right)-3\left(E h_{E^{m}} Y_{E}^{*}\right)_{i m}+6\left(\Lambda_{D^{q}} Y_{D}^{*} h_{U^{m}}^{t} \Lambda_{U^{m}}^{*}\right)_{i q} \\
& +3\left(\Lambda_{E^{n}}^{t} \Lambda_{D^{p}}^{*} h_{D^{p}}^{t} Y_{E}^{*}\right)_{i n}+3\left(E h_{D^{m}} Y_{D}^{*}\right)_{i m}-6\left(\Lambda_{D^{q}} Y_{D}^{*}\right)_{i l} \operatorname{Tr}\left(\Lambda_{D^{l}}^{*} h_{D^{q}}^{t}\right) \\
& +6\left(h_{D^{q}} Y_{D^{*}}^{*} \Lambda_{U^{m}}^{t} \Lambda_{U^{m}}^{*}\right)_{i q}-6\left(h_{D^{q}} Y_{D}^{*}\right)_{i l} \operatorname{Tr}\left(\Lambda_{D^{l}}^{*} \Lambda_{D^{q}}^{t}\right)-9\left(h_{D^{q}} D^{t} Y_{D}^{*}\right)_{i q} \\
& \left.-3\left(Y_{D^{\dagger}}^{T} h_{D^{n}}^{t}\right)_{n i}-3\left(h_{D^{n}} \Lambda_{D^{m}}^{\dagger} \Lambda_{D^{m}} Y_{D}^{*}+\Lambda_{D^{n}} \Lambda_{D^{m}}^{\dagger} h_{D^{m}} Y_{D}^{*}\right)_{i n}\right\} \\
& +\left(h_{D^{k}}\right)_{i j}\left\{\frac{15}{2} g_{2}^{4}+\frac{287}{90} g_{1}^{4}-\frac{16}{9} g_{3}^{4}+8 g_{3}^{2} g_{2}^{2}+\frac{8}{9} g_{3}^{2} g_{1}^{2}+g_{2}^{2} g_{1}^{2}\right\} \\
& +\left(\Lambda_{D^{k}}\right)_{i j}\left\{-30 M_{2} g_{2}^{2}-\frac{574}{45} M_{1} g_{1}^{4}+\frac{64}{9} M_{3} g_{3}^{4}-16\left(M_{3}+M_{2}\right) g_{3}^{2} g_{2}^{2}\right. \\
& \left.-\frac{16}{9}\left(M_{3}+M_{1}\right) g_{3}^{2} g_{1}^{2}-2\left(M_{1}+M_{2}\right) g_{2}^{2} g_{1}^{2}\right\}
\end{aligned}
$$

$$
\begin{aligned}
& \left(16 \pi^{2}\right)^{2} \beta_{\left(h_{U^{i}}\right)_{j k}}^{(2)}=\left(h_{U^{l}}\right)_{j k}\left\{\left(6 g_{2}^{2}-\frac{2}{5} g_{1}^{2}\right)(\tilde{T})_{l i}+\left(\frac{8}{3} g_{3}^{2}-\frac{4}{15} g_{1}^{2}\right) \operatorname{Tr}\left(\Lambda_{U^{i}} \Lambda_{U^{l}}^{\dagger}\right)\right. \\
& -2\left(Y_{U}^{\dagger} D Y_{U}\right)_{l i}-6(\tilde{T})_{l i} \operatorname{Tr}(T)-4 \operatorname{Tr}\left(\Lambda_{U^{i}} \Lambda_{U^{l}}^{\dagger} \Lambda_{U^{p}} \Lambda_{U^{p}}^{\dagger}\right) \\
& -2\left(Y_{U^{\dagger}}^{\dagger} \Lambda_{D^{m}}^{t} \Lambda_{D^{m}}^{*} Y_{U}\right)_{l i}-4\left(\Lambda_{U^{l}}^{\dagger} \Lambda_{U^{i}}\right)_{q m} \operatorname{Tr}\left(\Lambda_{D^{m}}^{\dagger} \Lambda_{D^{q}}\right)-2\left(Y_{U}^{\dagger} T Y_{U}\right)_{l i} \\
& \left.-4 \operatorname{Tr}\left(\Lambda_{U^{l}}^{\dagger} \Lambda_{U^{i}} \tilde{D}\right)\right\}+\left(h_{U^{i}}\right)_{l k}\left\{\left(\frac{16}{3} g_{3}^{2}+\frac{16}{15} g_{1}^{2}\right)\left(\Lambda_{U^{q}} \Lambda_{U^{q}}^{\dagger}\right)_{j l}\right. \\
& +\left(6 g_{2}^{2}+\frac{2}{5} g_{1}^{2}\right)\left[(D)_{j l}+\operatorname{Tr}\left(\Lambda_{D^{j}} \Lambda_{D^{l}}^{\dagger}\right)\right]-2\left(Y_{D}^{\dagger} D Y_{D}+Y_{D}^{\dagger} T Y_{D}\right)_{l j} \\
& -2\left(Y_{D}^{t} \Lambda_{D^{p}}^{\dagger} \Lambda_{D^{p}} Y_{D}^{*}\right)_{j l}-6 \operatorname{Tr}\left(\Lambda_{D^{q}} \Lambda_{D^{q}}^{\dagger} \Lambda_{D^{j}} \Lambda_{D^{l}}^{\dagger}\right)-\left(6 Y_{D}^{\dagger} \Lambda_{D^{j}}^{t} \Lambda_{D^{p}}^{*} Y_{D}\right)_{l p} \\
& -2 \operatorname{Tr}\left[\Lambda_{D^{j}}^{t} \Lambda_{D^{l}}^{*}(D+T)+\Lambda_{D^{l}}^{\dagger} \Lambda_{D^{j}} \Lambda_{D^{q}}^{\dagger} \Lambda_{D^{q}}\right]-2 \tilde{D}_{l j} \operatorname{Tr}(E+3 D) \\
& +2 \operatorname{Tr}\left(\Lambda_{E^{q}} \Lambda_{E^{q}}^{\dagger} \Lambda_{D^{j}} \Lambda_{D^{l}}^{\dagger}+E \Lambda_{D^{j}} \Lambda_{D^{l}}^{\dagger}\right)-4\left(\Lambda_{U^{m}} \tilde{D} \Lambda_{U^{m}}^{\dagger}\right)_{j l} \\
& -4\left(\Lambda_{U^{m}}^{\dagger} \Lambda_{U^{p}} \Lambda_{U^{p}}^{\dagger} \Lambda_{U^{m}}\right)_{l j}+4\left(\Lambda_{U^{m}}^{\dagger}\right)_{l q}\left(\Lambda_{U^{m}}\right)_{j n} \operatorname{Tr}\left(\Lambda_{D^{n}} \Lambda_{D^{q}}^{\dagger}\right) \\
& -2\left(Y_{D}^{\dagger} \Lambda_{D^{j}}^{t} \Lambda_{E^{p}}^{*} Y_{E}\right)_{l p}-\left(6 Y_{D}^{t} \Lambda_{D^{l}}^{\dagger} \Lambda_{D^{p}} Y_{D}^{*}+2 Y_{D}^{t} \Lambda_{D^{l}}^{\dagger} \Lambda_{E^{p}} Y_{E}^{*}\right)_{j p} \\
& \left.-2\left(\Lambda_{U^{q}}^{\dagger} \Lambda_{U^{n}}\right)_{l j}\left[\operatorname{Tr}\left(\Lambda_{U^{n}}^{\dagger} \Lambda_{U^{q}}\right)+2\left(Y_{U}^{\dagger} Y_{U}\right)_{n q}\right]\right\} \\
& +\left(h_{U^{i}}\right)_{j l}\left\{\left(\frac{16}{3} g_{3}^{2}+\frac{16}{15} g_{1}^{2}\right)\left(\Lambda_{U^{q}} \Lambda_{U^{q}}^{\dagger}\right)_{k l}+\left(6 g_{2}^{2}+\frac{2}{5} g_{1}^{2}\right)\left[D_{k l}\right.\right. \\
& \left.+\operatorname{Tr}\left(\Lambda_{D^{k}} \Lambda_{D^{l}}^{\dagger}\right)\right]-2\left(Y_{D}^{\dagger} D Y_{D}\right)_{l k}-2\left(Y_{D}^{\dagger} T Y_{D}\right)_{l k} \\
& -2 \tilde{D}_{l k} \operatorname{Tr}(E+3 D)-2\left(Y_{D}^{t} \Lambda_{D^{p}}^{\dagger} \Lambda_{D^{p}} Y_{D}^{*}\right)_{k l} \\
& -2 \operatorname{Tr}\left[\Lambda_{D^{k}}^{t} \Lambda_{D^{l}}^{*}(D+T)\right]-2 \operatorname{Tr}\left(\Lambda_{D^{l}}^{\dagger} \Lambda_{D^{k}} \Lambda_{D^{q}}^{\dagger} \Lambda_{D^{q}}\right) \\
& -\operatorname{Tr}\left(6 \Lambda_{D^{q}} \Lambda_{D^{q}}^{\dagger} \Lambda_{D^{k}} \Lambda_{D^{l}}^{\dagger}+2 \Lambda_{E^{q}} \Lambda_{E^{q}}^{\dagger} \Lambda_{D^{k}} \Lambda_{D^{l}}^{\dagger}+2 E \Lambda_{D^{k}} \Lambda_{D^{l}}^{\dagger}\right) \\
& -4\left(\Lambda_{U^{m}} \tilde{D} \Lambda_{U^{m}}^{\dagger}\right)_{k l}-4\left(\Lambda_{U^{m}}^{\dagger} \Lambda_{U^{p}} \Lambda_{U^{p}}^{\dagger} \Lambda_{U^{m}}\right)_{l k}-6\left(Y_{D}^{\dagger} \Lambda_{D^{k}}^{t} \Lambda_{D^{p}}^{*} Y_{D}\right)_{l p} \\
& +4\left(\Lambda_{U^{m}}^{\dagger}\right)_{l q}\left(\Lambda_{U^{m}}\right)_{k n} \operatorname{Tr}\left(\Lambda_{D^{n}} \Lambda_{D^{q}}^{\dagger}\right)-\left(6 Y_{D}^{t} \Lambda_{D^{l}}^{\dagger} \Lambda_{D^{p}} Y_{D}^{*}+2 Y_{D}^{t} \Lambda_{D^{l}}^{\dagger} \Lambda_{E^{p}} Y_{E}^{*}\right)_{k p} \\
& \left.-2\left(Y_{D}^{\dagger} \Lambda_{D^{k}}^{t} \Lambda_{E^{p}}^{*} Y_{E}\right)_{l p}-2\left(\Lambda_{U^{q}}^{\dagger} \Lambda_{U^{n}}\right)_{l k}\left[\operatorname{Tr}\left(\Lambda_{U^{n}}^{\dagger} \Lambda_{U^{q}}\right)+2(\tilde{T})_{n q}\right]\right\} \\
& -2\left(\Lambda_{U^{l}}\right)_{j k}\left\{\left(6 M_{2} g_{2}^{2}-\frac{2}{5} M_{1} g_{1}^{2}\right)(\tilde{T})_{l i}-\left(6 g_{2}^{2}-\frac{2}{5} g_{1}^{2}\right)\left(Y_{U}^{\dagger} h_{U}\right)_{l i}\right. \\
& -\left(\frac{8}{3} g_{3}^{2}-\frac{4}{15} g_{1}^{2}\right) \operatorname{Tr}\left(h_{U^{i}} \Lambda_{U^{l}}^{\dagger}\right)+\left(\frac{8}{3} M_{3} g_{3}^{2}-\frac{4}{15} M_{1} g_{1}^{2}\right) \operatorname{Tr}\left(\Lambda_{U^{i}} \Lambda_{U^{l}}^{\dagger}\right) \\
& +2\left(Y_{U}^{\dagger} h_{U} \tilde{T}+Y_{U}^{\dagger} T h_{U}+Y_{U}^{\dagger} D h_{U}\right)_{l i}+2\left(Y_{U}^{\dagger} \Lambda_{D^{m}}^{t} \Lambda_{D^{m}}^{*} h_{U}\right)_{l i} \\
& +6\left(Y_{U}^{\dagger} h_{U}\right)_{l i} \operatorname{Tr}(T)+6(\tilde{T})_{l i} \operatorname{Tr}\left(h_{U} Y_{U}^{\dagger}\right)+2\left(Y_{U}^{\dagger} h_{D} Y_{D}^{\dagger} Y_{U}\right)_{l i} \\
& +4 \operatorname{Tr}\left(\Lambda_{U^{l}}^{\dagger} \Lambda_{U^{i}} Y_{D}^{\dagger} h_{D}\right)+4\left(\Lambda_{U^{l}}^{\dagger} h_{U^{i}}\right)_{n m} \operatorname{Tr}\left(\Lambda_{D^{m}}^{\dagger} \Lambda_{D^{n}}\right)
\end{aligned}
$$




$$
\begin{aligned}
& +4 \operatorname{Tr}\left(h_{U^{i}} \Lambda_{U^{l}}^{\dagger} \Lambda_{U^{p}} \Lambda_{U^{p}}^{\dagger}\right)+4 \operatorname{Tr}\left(\Lambda_{U^{i}} \Lambda_{U^{l}}^{\dagger} h_{U^{p}} \Lambda_{U^{p}}^{\dagger}\right) \\
& \left.+4 \operatorname{Tr}\left(\Lambda_{U^{l}}^{\dagger} h_{U^{i}} \tilde{D}\right)+2\left(Y_{U}^{\dagger} h_{D^{m}}^{t} \Lambda_{D^{m}}^{*} Y_{U}\right)_{l i}+4\left(\Lambda_{U^{l}}^{\dagger} \Lambda_{U^{i}}\right)_{n m} \operatorname{Tr}\left(\Lambda_{D^{m}}^{\dagger} h_{D^{n}}\right)\right\} \\
& -2\left(\Lambda_{U^{i}}\right)_{l k}\left\{\left(\frac{16}{3} M_{3} g_{3}^{2}+\frac{16}{15} M_{1} g_{1}^{2}\right)\left(\Lambda_{U^{q}} \Lambda_{U^{q}}^{\dagger}\right)_{j l}+2\left(Y_{D^{\dagger}}^{\dagger} \Lambda_{D^{j}}^{t} \Lambda_{E^{p}}^{*} h_{E}\right)_{l p}\right. \\
& -\left(6 g_{2}^{2}+\frac{2}{5} g_{1}^{2}\right)\left[\left(h_{D} Y_{D}^{\dagger}\right)_{j l}+\operatorname{Tr}\left(h_{D^{j}} \Lambda_{D^{l}}^{\dagger}\right)\right]+2 \operatorname{Tr}\left(h_{E} Y_{E}^{\dagger} \Lambda_{D^{j}} \Lambda_{D^{l}}^{\dagger}\right) \\
& +\left(6 M_{2} g_{2}^{2}+\frac{2}{5} M_{1} g_{1}^{2}\right)\left[(D)_{j l}+\operatorname{Tr}\left(\Lambda_{D^{j}} \Lambda_{D^{l}}^{\dagger}\right)\right]-\left(\frac{16}{3} g_{3}^{2}+\frac{16}{15} g_{1}^{2}\right)\left(h_{U^{q}} \Lambda_{U^{q}}^{\dagger}\right)_{j l} \\
& +2 \tilde{D}_{l j} \operatorname{Tr}\left(Y_{E}^{\dagger} h_{E}\right)+2 \operatorname{Tr}\left(\Lambda_{D^{j}}^{t} \Lambda_{D^{l}}^{*} h_{U} Y_{U}^{\dagger}\right)+4\left(\Lambda_{U^{q}}^{\dagger} \Lambda_{U^{n}}\right)_{l j}\left(Y_{U}^{\dagger} h_{U}\right)_{n q} \\
& +2\left(Y_{D}^{\dagger} h_{D} \tilde{D}+Y_{D}^{\dagger} h_{U} Y_{U}^{\dagger} Y_{D}\right)_{l j}+2\left(Y_{D}^{\dagger} D h_{D}+Y_{D}^{\dagger} T h_{D}\right)_{l j} \\
& +2\left(Y_{D}^{\dagger} h_{D}\right)_{l j} \operatorname{Tr}(E+3 D)+6 \tilde{D}_{l j} \operatorname{Tr}\left(Y_{D}^{\dagger} h_{D}\right) \\
& +2\left(h_{D}^{t} \Lambda_{D^{p}}^{\dagger} \Lambda_{D^{p}} Y_{D}^{*}\right)_{j l}+2 \operatorname{Tr}\left(\Lambda_{D^{j}}^{t} \Lambda_{D^{l}}^{*} h_{D} Y_{D}^{\dagger}\right)+4\left(\Lambda_{U^{m}} Y_{D}^{\dagger} h_{D} \Lambda_{U^{m}}^{\dagger}\right)_{j l} \\
& +6\left(Y_{D}^{\dagger} \Lambda_{D^{j}}^{t} \Lambda_{D^{p}}^{*} h_{D}\right)_{l p}+\left(6 h_{D}^{t} \Lambda_{D^{l}}^{\dagger} \Lambda_{D^{p}} Y_{D}^{*}+2 h_{D}^{t} \Lambda_{D^{l}}^{\dagger} \Lambda_{E^{p}} Y_{E}^{*}\right)_{j p} \\
& +2 \operatorname{Tr}\left(h_{E^{q}} \Lambda_{E^{q}}^{\dagger} \Lambda_{D^{j}} \Lambda_{D^{l}}^{\dagger}\right)+2\left(Y_{D}^{t} \Lambda_{D^{l}}^{\dagger} h_{E^{p}} Y_{E}^{*}\right)_{j p}+4\left(h_{U^{m}} \tilde{D} \Lambda_{U^{m}}^{\dagger}\right)_{j l} \\
& +4\left(\Lambda_{U^{m}}^{\dagger} h_{U^{p}} \Lambda_{U^{p}}^{\dagger} \Lambda_{U^{m}}\right)_{l j}+4\left(\Lambda_{U^{m}}^{\dagger} \Lambda_{U^{p}} \Lambda_{U^{p}}^{\dagger} h_{U^{m}}\right)_{l j}-4\left(\Lambda_{U^{m}}^{\dagger}\right)_{l q}\left(h_{U^{m}}\right)_{j n} \operatorname{Tr}\left(\Lambda_{D^{n}} \Lambda_{D^{q}}^{\dagger}\right) \\
& +2\left(\Lambda_{U^{q}}^{\dagger} h_{U^{n}}\right)_{l j}\left[\operatorname{Tr}\left(\Lambda_{U^{n}}^{\dagger} \Lambda_{U^{q}}\right)+2(\tilde{T})_{n q}\right]+2\left(\Lambda_{U^{q}}^{\dagger} \Lambda_{U^{n}}\right)_{l j} \operatorname{Tr}\left(\Lambda_{U^{n}}^{\dagger} h_{U^{q}}\right) \\
& +2\left(Y_{D}^{t} \Lambda_{D^{p}}^{\dagger} h_{D^{p}} Y_{D}^{*}\right)_{j l}+2 \operatorname{Tr}\left[h_{D^{j}}^{t} \Lambda_{D^{l}}^{*}(D+T)+\Lambda_{D^{l}}^{\dagger} h_{D^{j}} \Lambda_{D^{q}}^{\dagger} \Lambda_{D^{q}}\right. \\
& \left.+\Lambda_{D^{l}}^{\dagger} \Lambda_{D^{j}} \Lambda_{D^{q}}^{\dagger} h_{D^{q}}\right]+6 \operatorname{Tr}\left(h_{D^{q}} \Lambda_{D^{q}}^{\dagger} \Lambda_{D^{j}} \Lambda_{D^{l}}^{\dagger}+\Lambda_{D^{q}} \Lambda_{D^{q}}^{\dagger} h_{D^{j}} \Lambda_{D^{l}}^{\dagger}\right) \\
& +2 \operatorname{Tr}\left(\Lambda_{E^{q}} \Lambda_{E^{q}}^{\dagger} h_{D^{j}} \Lambda_{D^{l}}^{\dagger}+E h_{D^{j}} \Lambda_{D^{l}}^{\dagger}\right)-4\left(\Lambda_{U^{m}}^{\dagger}\right)_{l q}\left(\Lambda_{U^{m}}\right)_{j n} \operatorname{Tr}\left(h_{D^{n}} \Lambda_{D^{q}}^{\dagger}\right) \\
& \left.+\left(6 Y_{D^{\dagger}}^{\dagger} h_{D^{j}}^{t} \Lambda_{D^{p}}^{*} Y_{D}\right)_{l_{p}}+2\left(Y_{D^{\dagger}}^{\dagger} h_{D^{j}}^{t} \Lambda_{E^{p}}^{*} Y_{E}\right)_{l_{p}}+\left(6 Y_{D}^{t} \Lambda_{D^{l}}^{\dagger} h_{D^{p}} Y_{D}^{*}\right)_{j p}\right\} \\
& -2\left(\Lambda_{U^{i}}\right)_{j l}\left\{\left(\frac{16}{3} M_{3} g_{3}^{2}+\frac{16}{15} M_{1} g_{1}^{2}\right)\left(\Lambda_{U^{q}} \Lambda_{U^{q}}^{\dagger}\right)_{k l}-\left(6 g_{2}^{2}+\frac{2}{5} g_{1}^{2}\right)\left(h_{D} Y_{D}^{\dagger}\right)_{k l}\right. \\
& +\left(6 M_{2} g_{2}^{2}+\frac{2}{5} M_{1} g_{1}^{2}\right)\left[(D)_{k l}+\operatorname{Tr}\left(\Lambda_{D^{k}} \Lambda_{D^{l}}^{\dagger}\right)\right]-\left(\frac{16}{3} g_{3}^{2}+\frac{16}{15} g_{1}^{2}\right)\left(h_{U^{q}} \Lambda_{U^{q}}^{\dagger}\right)_{k l} \\
& +2(\tilde{D})_{l k} \operatorname{Tr}\left(Y_{E}^{\dagger} h_{E}\right)+\operatorname{Tr}\left(2 h_{E} Y_{E}^{\dagger} \Lambda_{D^{k}} \Lambda_{D^{l}}^{\dagger}\right)+2\left(Y_{D^{\dagger}}^{\dagger} \Lambda_{D^{k}}^{t} \Lambda_{E^{p}}^{*} h_{E}\right)_{l p} \\
& +2\left(Y_{D}^{\dagger} h_{U} Y_{U}^{\dagger} Y_{D}\right)_{l k}+2 \operatorname{Tr}\left(\Lambda_{D^{k}}^{t} \Lambda_{D^{l}}^{*} h_{U} Y_{U}^{\dagger}\right)+4\left(\Lambda_{U^{q}}^{\dagger} \Lambda_{U^{n}}\right)_{l k}\left(Y_{U}^{\dagger} h_{U}\right)_{n q} \\
& +2\left(Y_{D}^{\dagger} h_{D} \tilde{D}\right)_{l k}+2\left(Y_{D}^{\dagger} D h_{D}\right)_{l k}+2\left(Y_{D}^{\dagger} T h_{D}\right)_{l k} \\
& +6(\tilde{D})_{l k} \operatorname{Tr}\left(Y_{D}^{\dagger} h_{D}\right)+2\left(h_{D}^{t} \Lambda_{D^{p}}^{\dagger} \Lambda_{D^{p}} Y_{D}^{*}\right)_{k l}+2 \operatorname{Tr}\left(\Lambda_{D^{k}}^{t} \Lambda_{D^{l}}^{*} h_{D} Y_{D}^{\dagger}\right) \\
& +4\left(\Lambda_{U^{m}} Y_{D}^{\dagger} h_{D} \Lambda_{U^{m}}^{\dagger}\right)_{k l}+6\left(Y_{D}^{\dagger} \Lambda_{D^{k}}^{t} \Lambda_{D^{p}}^{*} h_{D}\right)_{l p}+6\left(h_{D}^{t} \Lambda_{D^{l}}^{\dagger} \Lambda_{D^{p}} Y_{D}^{*}\right. \\
& \left.+2 h_{D}^{t} \Lambda_{D^{l}}^{\dagger} \Lambda_{E^{p}} Y_{E}^{*}\right)_{k p}+2 \operatorname{Tr}\left(h_{E q} \Lambda_{E^{q}}^{\dagger} \Lambda_{D^{k}} \Lambda_{D^{l}}^{\dagger}\right)+2\left(Y_{D}^{t} \Lambda_{D^{l}}^{\dagger} h_{E^{p}} Y_{E}^{*}\right)_{k p} \\
& +4\left(h_{U^{m}} \tilde{D} \Lambda_{U^{m}}^{\dagger}\right)_{k l}+4\left(\Lambda_{U^{m}}^{\dagger} h_{U^{p}} \Lambda_{U^{p}}^{\dagger} \Lambda_{U^{m}}\right)_{l k}+4\left(\Lambda_{U^{m}}^{\dagger} \Lambda_{U^{p}} \Lambda_{U^{p}}^{\dagger} h_{U^{m}}\right)_{l k} \\
& -4\left(\Lambda_{U^{m}}^{\dagger}\right)_{l q}\left(h_{U^{m}}\right)_{k n} \operatorname{Tr}\left(\Lambda_{D^{n}} \Lambda_{D^{q}}^{\dagger}\right)+2\left(\Lambda_{U^{q}}^{\dagger} h_{U^{n}}\right)_{l k}\left[\operatorname{Tr}\left(\Lambda_{U^{n}}^{\dagger} \Lambda_{U^{q}}\right)+2(\tilde{T})_{n q}\right] \\
& +2\left(\Lambda_{U^{q}}^{\dagger} \Lambda_{U^{n}}\right)_{l k} \operatorname{Tr}\left(\Lambda_{U^{n}}^{\dagger} h_{U^{q}}\right)+2\left(Y_{D^{t}}^{t} \Lambda_{D^{p}}^{\dagger} h_{D^{p}} Y_{D}^{*}\right)_{k l}+2 \operatorname{Tr}\left[h_{D^{k}}^{t} \Lambda_{D^{l}}^{*}(D+T)\right] \\
& +2 \operatorname{Tr}\left(\Lambda_{D^{l}}^{\dagger} h_{D^{k}} \Lambda_{D^{q}}^{\dagger} \Lambda_{D^{q}}+\Lambda_{D^{l}}^{\dagger} \Lambda_{D^{k}} \Lambda_{D^{q}}^{\dagger} h_{D^{q}}\right)+\operatorname{Tr}\left(6 h_{D^{q}} \Lambda_{D^{q}}^{\dagger} \Lambda_{D^{k}} \Lambda_{D^{l}}^{\dagger}\right. \\
& \left.+6 \Lambda_{D^{q}} \Lambda_{D^{q}}^{\dagger} h_{D^{k}} \Lambda_{D^{l}}^{\dagger}+2 \Lambda_{E^{q}} \Lambda_{E^{q}}^{\dagger} h_{D^{k}} \Lambda_{D^{l}}^{\dagger}+2 E h_{D^{k}} \Lambda_{D^{l}}^{\dagger}\right) \\
& -4\left(\Lambda_{U^{m}}^{\dagger}\right)_{l q}\left(\Lambda_{U^{m}}\right)_{k n} \operatorname{Tr}\left(h_{D^{n}} \Lambda_{D^{q}}^{\dagger}\right)+6\left(Y_{D}^{\dagger} h_{D^{k}}^{t} \Lambda_{D^{p}}^{*} Y_{D}\right)_{l p}+2\left(Y_{D^{\dagger}}^{\dagger} h_{D^{k}}^{t} \Lambda_{E^{p}}^{*} Y_{E}\right)_{l p} \\
& \left.+6\left(Y_{D}^{t} \Lambda_{D^{l}}^{\dagger} h_{D^{p}} Y_{D}^{*}\right)_{k p}+2\left(Y_{D}^{\dagger} h_{D}\right)_{l k} \operatorname{Tr}(E+3 D)\right\} \\
& +\left(\Lambda_{U^{i}}\right)_{j k}\left(\frac{32}{3} M_{3} g_{3}^{4}-\frac{112}{5} M_{1} g_{1}^{4}-\frac{128}{15}\left(M_{1}+M_{3}\right) g_{3}^{2} g_{1}^{2}\right)
\end{aligned}
$$




$$
+\left(h_{U^{i}}\right)_{j k}\left(-\frac{8}{3} g_{3}^{4}+\frac{28}{5} g_{1}^{4}+\frac{64}{15} g_{3}^{2} g_{1}^{2}\right)
$$

The two loop $\beta$ functions for the gaugino masses and soft breaking masses are given by:

$$
\begin{aligned}
& \left(16 \pi^{2}\right)^{2} \beta_{M_{1}}^{(2)}=\left(16 \pi^{2}\right)^{2} \beta_{M_{1(R P C)}^{(2)}}^{(2)}-2 M_{1} g_{1}^{2}\left\{\frac{12}{5} \operatorname{Tr}\left(\Lambda_{U^{i}} \Lambda_{U^{i}}^{\dagger}\right)+\frac{14}{5} \operatorname{Tr}\left(\Lambda_{D^{i}} h_{D^{i}}^{\dagger}\right)+\frac{9}{5} \operatorname{Tr}\left(\Lambda_{E^{i}} \Lambda_{E^{i}}^{\dagger}\right)\right\} \\
& +2 g_{1}^{2}\left[\frac{12}{5} \operatorname{Tr}\left(h_{U^{i}} \Lambda_{U^{i}}^{\dagger}\right)+\frac{14}{5} \operatorname{Tr}\left(h_{D^{i}} \Lambda_{D^{i}}^{\dagger}\right)+\frac{9}{5} \operatorname{Tr}\left(h_{E^{i}} \Lambda_{E^{i}}^{\dagger}\right)\right] \\
& \left(16 \pi^{2}\right)^{2} \beta_{M_{2}}^{(2)}=\left(16 \pi^{2}\right)^{2} \beta_{M_{2}(R P C)}^{(2)}-2 M_{2} g_{2}^{2}\left[6 \operatorname{Tr}\left(\Lambda_{D^{i}} \Lambda_{D^{i}}^{\dagger}\right)+\operatorname{Tr}\left(\Lambda_{E^{i}} \Lambda_{E^{i}}^{\dagger}\right)\right] \\
& +2 g_{2}^{2}\left[6 \operatorname{Tr}\left(h_{D^{i}} \Lambda_{D^{i}}^{\dagger}\right)+\operatorname{Tr}\left(h_{E^{i}} \Lambda_{E^{i}}^{\dagger}\right)\right] \\
& \left(16 \pi^{2}\right)^{2} \beta_{M_{3}}^{(2)}=\left(16 \pi^{2}\right)^{2} \beta_{M_{3(R P C)}}^{(2)}-2 M_{3} g_{3}^{2}\left[3 \operatorname{Tr}\left(\Lambda_{U^{i}} \Lambda_{U^{i}}^{\dagger}\right)+4 \operatorname{Tr}\left(\Lambda_{D^{i}} \Lambda_{D^{i}}^{\dagger}\right)\right] \\
& +2 g_{3}^{2}\left[3 \operatorname{Tr}\left(h_{U^{i}} \Lambda_{U^{i}}^{\dagger}\right)+4 \operatorname{Tr}\left(h_{D^{i}} \Lambda_{D^{i}}^{\dagger}\right)\right] \\
& \left(16 \pi^{2}\right)^{2} \beta_{\left(M_{\tilde{E}}^{2}\right)_{i j}}^{(2)}=\left(16 \pi^{2}\right)^{2} \beta_{\left(M_{\tilde{E}}^{2}\right)_{R P C}}^{(2)}+\left(12 M_{2}^{2} g_{2}^{2}-\frac{12}{5} M_{1}^{2} g_{1}^{2}\right) \operatorname{Tr}\left(\Lambda_{E^{j}} \Lambda_{E^{i}}^{\dagger}\right)-\left(6 M_{2} g_{2}^{2}-\frac{6}{5} M_{1} g_{1}^{2}\right) \operatorname{Tr}\left(\Lambda_{E^{j}} h_{E^{i}}^{\dagger}\right) \\
& -\left(6 M_{2}^{*} g_{2}^{2}-\frac{6}{5} M_{1}^{*} g_{1}^{2}\right) \operatorname{Tr}\left(h_{E^{j}} \Lambda_{E^{i}}^{\dagger}\right)+\left(6 g_{2}^{2}-\frac{6}{5} g_{1}^{2}\right)\left[\operatorname{Tr}\left(h_{E^{j}} h_{E^{i}}^{\dagger}\right)\right. \\
& -4\left(h_{E}^{\dagger} h_{E^{l}} \Lambda_{E^{l}}^{\dagger} Y_{E}+h_{E}^{\dagger} \Lambda_{E^{l}} \Lambda_{E^{l}}^{\dagger} h_{E}+Y_{E}^{\dagger} h_{E^{l}} h_{E^{l}}^{\dagger} Y_{E}+Y_{E}^{\dagger} \Lambda_{E^{l}} h_{E^{l}}^{\dagger} h_{E}\right)_{i j} \\
& -12\left(h_{E}^{\dagger} h_{D^{l}} \Lambda_{D^{l}}^{\dagger} Y_{E}+h_{E}^{\dagger} \Lambda_{D^{l}} \Lambda_{D^{l}}^{\dagger} h_{E}+Y_{E}^{\dagger} h_{D^{l}} h_{D^{l}}^{\dagger} Y_{E}+Y_{E}^{\dagger} \Lambda_{D^{l}} h_{D^{l}}^{\dagger} h_{E}\right)_{i j} \\
& +12\left(h_{E}^{\dagger} h_{E^{j}} \Lambda_{D^{m}}^{*} Y_{D}+h_{E}^{\dagger} \Lambda_{E^{j}} \Lambda_{D^{m}}^{*} h_{D}+Y_{E}^{\dagger} h_{E^{j}} h_{D^{m}}^{*} Y_{D}+Y_{E}^{\dagger} \Lambda_{E^{j}} h_{D^{m}}^{*} h_{D}\right)_{i m} \\
& -4\left(h_{E}^{\dagger} \Lambda_{E^{j}} \Lambda_{E^{m}}^{\dagger} h_{E}+h_{E}^{\dagger} h_{E^{j}} \Lambda_{E^{m}}^{\dagger} Y_{E}+Y_{E}^{\dagger} h_{E^{j}} h_{E^{m}}^{\dagger} Y_{E}+Y_{E}^{\dagger} \Lambda_{E^{j}} h_{E^{m}}^{\dagger} h_{E}\right)_{i m} \\
& -4 \operatorname{Tr}\left[h_{E^{j}} h_{E^{i}}^{\dagger}\left(E+\Lambda_{E^{l}} \Lambda_{E^{l}}^{\dagger}+3 \Lambda_{D^{l}} \Lambda_{D^{l}}^{\dagger}\right)\right]+12\left(Y_{D}^{\dagger} h_{D^{m}}^{t} h_{E^{i}}^{\dagger} Y_{E}\right. \\
& \left.+Y_{D}^{\dagger} \Lambda_{D^{m}}^{t} h_{E^{i}}^{\dagger} h_{E}\right)_{m j}-4 \operatorname{Tr}\left[\Lambda_{E^{j}} h_{E^{i}}^{\dagger}\left(h_{E} Y_{E}^{\dagger}+h_{E^{l}} \Lambda_{E^{l}}^{\dagger}+3 h_{D^{l}} \Lambda_{D^{l}}^{\dagger}\right)\right] \\
& -4 \operatorname{Tr}\left[h_{E^{j}} \Lambda_{E^{i}}^{\dagger}\left(Y_{E} h_{E}^{\dagger}+\Lambda_{E^{l}} h_{E^{l}}^{\dagger}+3 \Lambda_{D^{l}} h_{D^{l}}^{\dagger}\right)\right]+12\left(h_{D}^{\dagger} h_{D^{m}}^{t} \Lambda_{E^{i}}^{\dagger} Y_{E}\right. \\
& \left.+h_{D}^{\dagger} \Lambda_{D^{m}}^{t} \Lambda_{E^{i}}^{\dagger} h_{E}\right)_{m j}-4 \operatorname{Tr}\left[\Lambda_{E^{j}} \Lambda_{E^{i}}^{\dagger}\left(h_{E} h_{E}^{\dagger}+h_{E^{l}} h_{E^{l}}^{\dagger}+3 h_{D^{l}} h_{D^{l}}^{\dagger}\right)\right] \\
& -4\left(h_{E}^{\dagger} h_{E^{m}} \Lambda_{E^{i}}^{\dagger} Y_{E}+h_{E}^{\dagger} \Lambda_{E^{m}} \Lambda_{E^{i}}^{\dagger} h_{E}+Y_{E}^{\dagger} h_{E^{m}} h_{E^{i}}^{\dagger} Y_{E}+Y_{E}^{\dagger} \Lambda_{E^{m}} h_{E^{i}}^{\dagger} h_{E}\right)_{m j} \\
& +\left(3 g_{2}^{2}-\frac{3}{5} g_{1}^{2}\right)\left[4 \operatorname{Tr}\left(m_{\tilde{L}}^{2} \Lambda_{E^{j}} \Lambda_{E^{i}}^{\dagger}\right)+\left(m_{\tilde{E}}^{2}\right)_{l j} \operatorname{Tr}\left(\Lambda_{E^{l}} \Lambda_{E^{i}}^{\dagger}\right)+\left(m_{\tilde{E}}^{2}\right)_{i l} \operatorname{Tr}\left(\Lambda_{E^{j}} \Lambda_{E^{l}}^{\dagger}\right)\right. \\
& \left.+4\left(\Lambda_{E^{i}}^{\dagger} Y_{E}\right)_{l j} m_{\tilde{L}_{l} H_{1}}^{2}\right]+\left(12 g_{2}^{2}-\frac{12}{5} g_{1}^{2}\right)\left(Y_{E}^{\dagger} \Lambda_{E^{j}}\right)_{i l} m_{H_{1} \tilde{L}_{l}}^{2}-2\left(Y_{E}^{\dagger} \Lambda_{E^{k}}\right)_{i q}(\tilde{E})_{k j} m_{H_{1} \tilde{L}_{q}}^{2} \\
& -4\left(Y_{E}^{\dagger} E \Lambda_{E^{j}}\right)_{i q} m_{H_{1} \tilde{L}_{q}}^{2}-4\left(Y_{E}^{\dagger} m_{\tilde{L}^{2}}^{2} \Lambda_{E^{l}} \Lambda_{E^{l}}^{\dagger} Y_{E}\right)_{i j}-4\left(Y_{E}^{\dagger} \Lambda_{E^{l}}\left(m_{\tilde{L}}^{2}\right)^{t} \Lambda_{E^{l}}^{\dagger} Y_{E}\right)_{i j} \\
& -4\left(m_{\tilde{E}}^{2}\right)_{k l}\left(Y_{E}^{\dagger} \Lambda_{E^{k}} \Lambda_{E^{l}}^{\dagger} Y_{E}\right)_{i j}-2(\tilde{E})_{i l}\left(\Lambda_{E^{l}}^{\dagger} Y_{E}\right)_{q j} m_{\tilde{L}_{q} H_{1}}^{2}+2\left(Y_{E}^{t} \Lambda_{E^{l}}^{\dagger} Y_{E}\right)_{l j}\left(Y_{E}^{\dagger}\right)_{i q} m_{\tilde{L}_{q} H_{1}}^{2} \\
& -4\left(Y_{E}^{\dagger} \Lambda_{E^{l}} \Lambda_{E^{l}}^{\dagger} m_{\tilde{L}}^{2} Y_{E}\right)_{i j}-2\left(Y_{E}^{\dagger} \Lambda_{E^{l}} \Lambda_{E^{l}}^{\dagger} Y_{E} m_{\tilde{E}}^{2}\right)_{i j}-4\left(Y_{E}^{\dagger} \Lambda_{E^{l}} \Lambda_{E^{l}}^{\dagger} \Lambda_{E^{j}}\right)_{i l} m_{H_{1} \tilde{L}_{l}}^{2} \\
& -4 m_{H_{1}}^{2}\left(Y_{E}^{\dagger} \Lambda_{E^{l}} \Lambda_{E^{l}}^{\dagger} Y_{E}\right)_{i j}-12\left(Y_{E}^{\dagger} m_{\tilde{L}}^{2} \Lambda_{D^{l}} \Lambda_{D^{l}}^{\dagger} Y_{E}+Y_{E}^{\dagger} \Lambda_{D^{l}}\left(m_{\tilde{Q}}^{2}\right)^{t} \Lambda_{D^{l}}^{\dagger} Y_{E}\right)_{i j} \\
& -12\left(m_{\tilde{D}}^{2}\right)_{k l}\left(Y_{E}^{\dagger} \Lambda_{D^{k}} \Lambda_{D^{l}}^{\dagger} Y_{E}\right)_{i j}+6\left(Y_{D}^{t} \Lambda_{D^{l}}^{\dagger} Y_{E}\right)_{l j}\left(Y_{E}^{\dagger}\right)_{i q} m_{\tilde{L}_{q} H_{1}}^{2} \\
& -12\left(Y_{E}^{\dagger} \Lambda_{D^{l}} \Lambda_{D^{l}}^{\dagger} m_{\tilde{L}}^{2} Y_{E}\right)_{i j}-12 m_{H_{1}}^{2}\left(Y_{E}^{\dagger} \Lambda_{D^{l}} \Lambda_{D^{l}}^{\dagger} Y_{E}\right)_{i j}-6\left(Y_{E}^{\dagger} \Lambda_{D^{l}} \Lambda_{D^{l}}^{\dagger} Y_{E} m_{\tilde{E}}^{2}\right)_{i j}
\end{aligned}
$$




$$
\begin{aligned}
& -4\left(Y_{E}^{\dagger} \Lambda_{E^{j}}\right)_{i l} m_{H_{1} \tilde{L}_{l}}^{2} \operatorname{Tr}(E+3 D)-4 \tilde{E}_{i j}\left(Y_{E^{\prime}}^{\dagger} \Lambda_{E^{k}}\right)_{k l} m_{H_{1} \tilde{L}_{l}}^{2} \\
& +12(\tilde{E})_{i j}\left(Y_{D}^{\dagger} \Lambda_{D^{k}}^{t}\right)_{k l} m_{H_{1} \tilde{L}_{l}}^{2}+12\left(Y_{E}^{\dagger} m_{\tilde{L}}^{2} \Lambda_{E^{j}} \Lambda_{D^{m}}^{*} Y_{D}\right)_{i m} \\
& +12\left(Y_{E}^{\dagger} \Lambda_{E^{j}} m_{\tilde{L}}^{2 t} \Lambda_{D^{m}}^{*} Y_{D}\right)_{i m}+6\left(m_{\tilde{E}}^{2}\right)_{k j}\left(Y_{E}^{\dagger} \Lambda_{E^{k}} \Lambda_{D^{m}}^{*} Y_{D}\right)_{i m} \\
& +6(\tilde{E})_{i j}\left(\Lambda_{D^{m}}^{*} Y_{D}\right)_{q m} m_{\tilde{L}_{q} H_{1}}^{2}-6\left(Y_{E}^{t} \Lambda_{D^{m}}^{*} Y_{D}\right)_{j m}\left(Y_{E}^{\dagger}\right)_{i l} m_{\tilde{L}_{l} H_{1}}^{2} \\
& +12\left(Y_{E}^{\dagger} \Lambda_{E^{j}} \Lambda_{D^{m}}^{*} m_{\tilde{Q}}^{2} Y_{D}\right)_{i m}+12\left(Y_{E}^{\dagger} \Lambda_{E^{j}} \Lambda_{D^{m}}^{*} Y_{D} m_{\tilde{D}}^{2}\right)_{i m}-4 m_{H_{1}}^{2} \operatorname{Tr}\left(\Lambda_{E^{j}} \Lambda_{E^{i}}^{\dagger} E\right) \\
& +12 m_{H_{1}}^{2}\left(Y_{E^{\dagger}}^{\dagger} \Lambda_{E^{j}} \Lambda_{D^{m}}^{*} Y_{D}\right)_{i m}-12\left(Y_{E}^{\dagger} \Lambda_{E^{j}} \Lambda_{D^{m}}^{*} \Lambda_{D^{m}}^{t}\right)_{i l} m_{H_{1} \tilde{L}_{l}}^{2} \\
& -4\left(Y_{E}^{\dagger} m_{\tilde{L}}^{2} \Lambda_{E^{j}} \Lambda_{E^{m}}^{\dagger} Y_{E}\right)_{i m}-4\left(Y_{E}^{\dagger} \Lambda_{E^{j}}\left(m_{\tilde{L}}^{2}\right)^{t} \Lambda_{E^{m}}^{\dagger} Y_{E}\right)_{i m} \\
& -2\left(m_{\tilde{E}}^{2}\right)_{k j}\left(Y_{E}^{\dagger} \Lambda_{E^{k}} \Lambda_{E^{m}}^{\dagger} Y_{E}\right)_{i m}-2(\tilde{E})_{i j}\left(\Lambda_{E^{m}}^{\dagger} Y_{E}\right)_{q m} m_{\tilde{L}_{q} H_{1}}^{2} \\
& +2\left(Y_{E}^{t} \Lambda_{E^{m}}^{\dagger} Y_{E}\right)_{j m}\left(Y_{E}^{\dagger}\right)_{i l} m_{\tilde{L}_{l} H_{1}}^{2}-4\left(Y_{E}^{\dagger} \Lambda_{E^{j}} \Lambda_{E^{m}}^{\dagger} m_{\tilde{L}}^{2} Y_{E}\right)_{i m} \\
& -4\left(Y_{E}^{\dagger} \Lambda_{E^{j}} \Lambda_{E^{m}}^{\dagger} Y_{E} m_{\tilde{E}}^{2}\right)_{i m}-4 m_{H_{1}}^{2}\left(Y_{E}^{\dagger} \Lambda_{E^{j}} \Lambda_{E m}^{\dagger} Y_{E}\right)_{i m}-4 \operatorname{Tr}\left(E m_{\tilde{L}}^{2} \Lambda_{E^{j}} \Lambda_{E^{i}}^{\dagger}\right) \\
& -4\left(Y_{E}^{\dagger} \Lambda_{E^{j}} \Lambda_{E^{m}}^{\dagger} \Lambda_{E^{m}}\right)_{i l} m_{H_{1} \tilde{L}_{l}}^{2}-12\left(Y_{E^{\prime}}^{\dagger} \Lambda_{D^{l}} \Lambda_{D^{l}}^{\dagger} \Lambda_{E^{j}}\right)_{i q} m_{H_{1} \tilde{L}_{q}}^{2} \\
& -4 \operatorname{Tr}\left(\Lambda_{E^{j}} m_{\tilde{L}}^{2 t} \Lambda_{E^{i}}^{\dagger} E\right)-2\left(m_{\tilde{E}}^{2}\right)_{l j} \operatorname{Tr}\left(\Lambda_{E^{l}} \Lambda_{E^{i}}^{\dagger} E\right)-4\left(\Lambda_{E^{i}}^{\dagger} E Y_{E}\right)_{q j} m_{\tilde{L}_{q} H_{1}}^{2} \\
& +2\left(Y_{E}^{t} \Lambda_{E^{i}}^{\dagger} E\right)_{j q} m_{\tilde{L}_{q} H_{1}}^{2}-4 \operatorname{Tr}\left(m_{\tilde{L}^{2}}^{2} \Lambda_{E^{j}} \Lambda_{E^{i}}^{\dagger} \Lambda_{E^{l}} \Lambda_{E^{l}}^{\dagger}\right)-4 \operatorname{Tr}\left(\Lambda_{E^{i}}^{\dagger} \Lambda_{E^{l}} \Lambda_{E^{l}}^{\dagger} \Lambda_{E^{j}}\left(m_{\tilde{L}}^{2}\right)^{t}\right) \\
& -2\left(m_{\tilde{E}}^{2}\right)_{k j} \operatorname{Tr}\left(\Lambda_{E^{k}} \Lambda_{E^{i}}^{\dagger} \Lambda_{E^{l}} \Lambda_{E^{l}}^{\dagger}\right)-4\left(\Lambda_{E^{i}}^{\dagger} \Lambda_{E^{l}} \Lambda_{E^{l}}^{\dagger} Y_{E}\right)_{q j} m_{\tilde{L}_{q} H_{1}}^{2} \\
& +2\left(Y_{E}^{t} \Lambda_{E^{i}}^{\dagger} \Lambda_{E^{l}} \Lambda_{E^{l}}^{\dagger}\right)_{j q} m_{\tilde{L}_{q} H_{1}}^{2}-12 \operatorname{Tr}\left(m_{\tilde{L}}^{2} \Lambda_{E^{j}} \Lambda_{E^{i}}^{\dagger} \Lambda_{D^{l}} \Lambda_{D^{l}}^{\dagger}\right)-12 \operatorname{Tr}\left(\Lambda_{E^{i}}^{\dagger} \Lambda_{D^{l}} \Lambda_{D^{l}}^{\dagger} \Lambda_{E^{j}}\left(m_{\tilde{L}}^{2}\right)^{t}\right) \\
& -6\left(m_{\tilde{E}}^{2}\right)_{k j} \operatorname{Tr}\left(\Lambda_{E^{k}} \Lambda_{E^{i}}^{\dagger} \Lambda_{D^{l}} \Lambda_{D^{l}}^{\dagger}\right)-12\left(\Lambda_{E^{i}}^{\dagger} \Lambda_{D^{l}} \Lambda_{D^{l}}^{\dagger} Y_{E}\right)_{q j} m_{\tilde{L}_{q} H_{1}}^{2}+6\left(Y_{E}^{t} \Lambda_{E^{i}}^{\dagger} \Lambda_{D^{l}} \Lambda_{D^{l}}^{\dagger}\right)_{j q} m_{\tilde{L}_{q} H_{1}}^{2} \\
& -4 \operatorname{Tr}\left(\Lambda_{E^{j}} \Lambda_{E^{i}}^{\dagger} m_{\tilde{L}}^{2} E\right)-4 \operatorname{Tr}\left(\Lambda_{E^{j}} \Lambda_{E^{i}}^{\dagger} Y_{E} m_{\tilde{E}}^{2} Y_{E}^{\dagger}\right)+2\left(Y_{E}^{\dagger} \Lambda_{E^{m}} Y_{E}^{*}\right)_{m i}\left(Y_{E}\right)_{q j} m_{H_{1} \tilde{L}_{q}}^{2} \\
& -4\left(Y_{E^{\prime}}^{\dagger} \Lambda_{E^{j}} \Lambda_{E^{i}}^{\dagger} \Lambda_{E^{k}}\right)_{k l} m_{H_{1} \tilde{L}_{l}}^{2}-4 \operatorname{Tr}\left(\Lambda_{E^{j}} \Lambda_{E^{i}}^{\dagger} m_{\tilde{L}^{2}}^{2} \Lambda_{E^{l}} \Lambda_{E^{l}}^{\dagger}\right)-4 \operatorname{Tr}\left(\Lambda_{E^{j}} \Lambda_{E^{i}}^{\dagger} \Lambda_{E^{l}}\left(m_{\tilde{L}}^{2}\right)^{t} \Lambda_{E^{l}}^{\dagger}\right) \\
& -4\left(m_{\tilde{E}}^{2}\right)_{k l} \operatorname{Tr}\left(\Lambda_{E^{j}} \Lambda_{E^{i}}^{\dagger} \Lambda_{E^{k}} \Lambda_{E^{l}}^{\dagger}\right)-4\left(\Lambda_{E^{l}}^{\dagger} \Lambda_{E^{j}} \Lambda_{E^{i}}^{\dagger} Y_{E}\right)_{q l} m_{\tilde{L}_{q} H_{1}}^{2} \\
& +2\left(Y_{E}^{t} \Lambda_{E^{l}}^{\dagger} \Lambda_{E^{j}} \Lambda_{E^{i}}^{\dagger}\right)_{l q} m_{\tilde{L}_{q} H_{1}}^{2}-12 \operatorname{Tr}\left(\Lambda_{E^{j}} \Lambda_{E^{i}}^{\dagger} m_{\tilde{L}}^{2} \Lambda_{D^{l}} \Lambda_{D^{l}}^{\dagger}\right) \\
& -12 \operatorname{Tr}\left(\Lambda_{E^{j}} \Lambda_{E^{i}}^{\dagger} \Lambda_{D^{l}}\left(m_{\tilde{Q}}^{2}\right)^{t} \Lambda_{D^{l}}^{\dagger}\right)-12\left(m_{\tilde{D}}^{2}\right)_{k l} \operatorname{Tr}\left(\Lambda_{E^{j}} \Lambda_{E^{i}}^{\dagger} \Lambda_{D^{k}} \Lambda_{D^{l}}^{\dagger}\right) \\
& +6\left(Y_{D}^{t} \Lambda_{D^{l}}^{\dagger} \Lambda_{E^{j}} \Lambda_{E^{i}}^{\dagger}\right)_{l q} m_{\tilde{L}_{q} H_{1}}^{2}+12\left(Y_{E}^{t} \Lambda_{E^{i}}^{*} m_{\tilde{L}^{2}}^{2} \Lambda_{D^{m}} Y_{D}^{*}\right)_{j m}+12\left(Y_{E}^{t} \Lambda_{E^{i}}^{*} \Lambda_{D^{m}}\left(m_{\tilde{Q}}^{2}\right)^{t} Y_{D}^{*}\right)_{j m} \\
& -12 \operatorname{Tr}(\tilde{D})\left(Y_{E}^{t} \Lambda_{E^{i}}^{*}\right)_{j l} m_{\tilde{L}_{l} H_{1}}^{2}+12\left(Y_{D}^{\dagger} \Lambda_{D^{m}}^{t} \Lambda_{E^{i}}^{\dagger} m_{\tilde{L}}^{2} Y_{E}\right)_{m j}+6\left(Y_{D}^{\dagger} \Lambda_{D^{m}}^{t} \Lambda_{E^{i}}^{\dagger} Y_{E} m_{\tilde{E}}^{2}\right)_{m j} \\
& +6\left(Y_{D}^{\dagger} \Lambda_{D^{m}}^{t} \Lambda_{E^{i}}^{\dagger} \Lambda_{E^{j}}\right)_{m l} m_{H_{1} \tilde{L}_{l}}^{2}+12 m_{H_{1}}^{2}\left(Y_{D^{\dagger}}^{\dagger} \Lambda_{D^{m}}^{t} \Lambda_{E^{i}}^{\dagger} Y_{E}\right)_{m j}-4\left(Y_{E}^{\dagger} m_{\tilde{L}}^{2} \Lambda_{E^{m}} \Lambda_{E^{i}}^{\dagger} Y_{E}\right)_{m j} \\
& -4\left(Y_{E}^{\dagger} \Lambda_{E^{m}}\left(m_{\tilde{L}}^{2}\right)^{t} \Lambda_{E^{i}}^{\dagger} Y_{E}\right)_{m j}-4\left(m_{\tilde{E}}^{2} Y_{E}^{\dagger} \Lambda_{E^{k}} \Lambda_{E^{i}}^{\dagger} Y_{E}\right)_{k j}-4 \operatorname{Tr}(\tilde{E})\left(\Lambda_{E^{i}}^{\dagger} Y_{E}\right)_{q j} m_{\tilde{L}_{q} H_{1}}^{2} \\
& -4\left(Y_{E}^{\dagger} \Lambda_{E^{m}} \Lambda_{E^{i}}^{\dagger} m_{\tilde{L}}^{2} Y_{E}\right)_{m j}-2\left(Y_{E}^{\dagger} \Lambda_{E^{m}} \Lambda_{E^{i}}^{\dagger} Y_{E} m_{\tilde{E}}^{2}\right)_{m j}-4\left(m_{H_{1}}^{2}\right)\left(Y_{E^{\prime}}^{\dagger} \Lambda_{E^{m}} \Lambda_{E^{i}}^{\dagger} Y_{E}\right)_{m j} \\
& -2\left(Y_{E}^{\dagger} \Lambda_{E^{m}} \Lambda_{E^{i}}^{\dagger} \Lambda_{E^{j}}\right)_{m k} m_{H_{1} \tilde{L}_{k}}^{2}+12\left(m_{\tilde{D}}^{2} Y_{D}^{\dagger} \Lambda_{D^{k}}^{t} \Lambda_{E^{i}}^{\dagger} Y_{E}\right)_{k j}+2\left(E^{t} \Lambda_{E^{i}}^{\dagger} Y_{E}\right)_{q j} m_{\tilde{L}_{q} H_{1}}^{2} \\
& -2(\tilde{E})_{i l}\left(\Lambda_{E^{l}}^{\dagger} Y_{E}\right)_{q j} m_{\tilde{L}_{q} H_{1}}^{2}-2\left(m_{\tilde{E}}^{2} Y_{E}^{\dagger} \Lambda_{E^{l}} \Lambda_{E^{l}}^{\dagger} Y_{E}\right)_{i j}-2(\tilde{E})_{l j}\left(Y_{E}^{\dagger} \Lambda_{E^{l}}\right)_{i q} m_{H_{1} \tilde{L}_{q}}^{2} \\
& -6\left(m_{\tilde{E}}^{2} Y_{E}^{\dagger} \Lambda_{D^{l}} \Lambda_{D^{l}}^{\dagger} Y_{E}\right)_{i j}+6\left(Y_{E}^{\dagger} \Lambda_{D^{l}} Y_{D}^{*}\right)_{i l}\left(Y_{E}\right)_{q j} m_{H_{1} \tilde{L}_{q}}^{2}-2(\tilde{E})_{i j}\left(\Lambda_{E^{k}}^{\dagger} Y_{E}\right)_{l k} m_{\tilde{L}_{l} H_{1}}^{2} \\
& +6(\tilde{E})_{i j}\left(\Lambda_{D^{k}}^{*} Y_{D}\right)_{l k} m_{\tilde{L}_{l} H_{1}}^{2}+6\left(m_{\tilde{E}}^{2} Y_{E}^{\dagger} \Lambda_{E^{j}} \Lambda_{D^{m}}^{*} Y_{D}\right)_{i m}+6\left(\Lambda_{E^{i}}^{\dagger} \Lambda_{E^{j}} \Lambda_{D^{m}}^{*} Y_{D}\right)_{l m} m_{\tilde{L}_{l} H_{1}}^{2} \\
& -2\left(m_{\tilde{E}}^{2} Y_{E}^{\dagger} \Lambda_{E^{j}} \Lambda_{E^{m}}^{\dagger} Y_{E}\right)_{i m}-2\left(\Lambda_{E^{i}}^{\dagger} \Lambda_{E^{j}} \Lambda_{E^{m}}^{\dagger} Y_{E}\right)_{l m} m_{\tilde{L}_{l} H_{1}}^{2}+2\left(Y_{E}^{\dagger} \Lambda_{E^{j}} E^{t}\right)_{i q} m_{H_{1} \tilde{L}_{q}}^{2} \\
& -2\left(m_{\tilde{E}}^{2}\right)_{i k} \operatorname{Tr}\left(\Lambda_{E^{j}} \Lambda_{E^{k}}^{\dagger} E\right)+2\left(E \Lambda_{E^{j}} Y_{E}^{*}\right)_{q i} m_{H_{1} \tilde{L}_{q}}^{2}-6\left(m_{\tilde{E}}^{2}\right)_{i k} \operatorname{Tr}\left(\Lambda_{E^{j}} \Lambda_{E^{k}}^{\dagger} \Lambda_{D^{l}} \Lambda_{D^{l}}^{\dagger}\right)
\end{aligned}
$$




$$
\begin{aligned}
& -2\left(m_{\tilde{E}}^{2}\right)_{i k} \operatorname{Tr}\left(\Lambda_{E^{j}} \Lambda_{E^{k}}^{\dagger} \Lambda_{E^{l}} \Lambda_{E^{l}}^{\dagger}\right)+2\left(\Lambda_{E^{j}} \Lambda_{E^{i}}^{\dagger} \Lambda_{E^{l}} Y_{E}^{*}\right)_{q l} m_{H_{1} \tilde{L}_{q}}^{2} \\
& +6\left(\Lambda_{E^{j}} \Lambda_{E^{i}}^{\dagger} \Lambda_{D^{l}} Y_{D}^{*}\right)_{q l} m_{H_{1} \tilde{L}_{q}}^{2}-6\left(\Lambda_{D^{m}}^{*} \Lambda_{D^{m}}^{t} \Lambda_{E^{i}}^{\dagger} Y_{E}\right)_{l j} m_{\tilde{L}_{l} H_{1}}^{2} \\
& +6\left(m_{\tilde{E}^{2}}^{2}\right)_{i k}\left(Y_{D^{\prime}}^{\dagger} \Lambda_{D^{m}}^{t} \Lambda_{E^{k}}^{\dagger} Y_{E}\right)_{m j}-6\left(Y_{D^{\dagger}}^{\dagger} \Lambda_{D^{m}}^{t} Y_{E}^{*}\right)_{m i}\left(Y_{E}\right)_{q j} m_{H_{1} \tilde{L}_{q}}^{2} \\
& -2\left(\Lambda_{E^{m}}^{\dagger} \Lambda_{E^{m}} \Lambda_{E^{i}}^{\dagger} Y_{E}\right)_{l j} m_{\tilde{L}_{l} H_{1}}^{2}-2\left(Y_{E}^{\dagger} \Lambda_{E^{m}} \Lambda_{E^{k}}^{\dagger} Y_{E}\right)_{m j}\left(m_{\tilde{E}}^{2}\right)_{i k}-\frac{6}{5} s^{\prime} \delta_{i j}
\end{aligned}
$$

$$
\begin{aligned}
& \left(16 \pi^{2}\right)^{2} \beta_{\left(M_{\tilde{L}}^{2}\right)_{i j}}^{(2)}=\left(16 \pi^{2}\right)^{2} \beta_{\left(M_{\tilde{L}}^{2}\right)_{R P C}}^{(2)}+ \\
& \left(64 M_{3}^{2} g_{3}^{2}-\frac{8}{5} M_{1}^{2} g_{1}^{2}\right)\left(\Lambda_{D^{q}} \Lambda_{D^{q}}^{\dagger}\right)_{i j}+\frac{24}{5} M_{1}^{2} g_{1}^{2}\left(\Lambda_{E^{q}} \Lambda_{E^{q}}^{\dagger}\right)_{i j} \\
& +\left(-32 M_{3} g_{3}^{2}+\frac{4}{5} M_{1} g_{1}^{2}\right)\left(\Lambda_{D^{q}} h_{D^{q}}^{\dagger}\right)_{i j}-\frac{12}{5} M_{1} g_{1}^{2}\left(\Lambda_{E^{q}} h_{E^{q}}^{\dagger}\right)_{i j} \\
& +\left(-32 M_{3}^{*} g_{3}^{2}+\frac{4}{5} M_{1}^{*} g_{1}^{2}\right)\left(h_{D^{q}} \Lambda_{D^{q}}^{\dagger}\right)_{i j}-\frac{12}{5} M_{1}^{*} g_{1}^{2}\left(h_{E^{q}} \Lambda_{E^{q}}^{\dagger}\right)_{i j} \\
& +\left(32 g_{3}^{2}-\frac{4}{5} g_{1}^{2}\right)\left(h_{D^{q}} h_{D^{q}}^{\dagger}\right)_{i j}+\frac{12}{5} g_{1}^{2}\left(h_{E^{q}} h_{E^{q}}^{\dagger}\right)_{i j} \\
& -2\left(h_{E}^{\dagger}\right)_{k j}\left(h_{E}\right)_{i l} \operatorname{Tr}\left(\Lambda_{E^{l}}^{\dagger} \Lambda_{E^{k}}\right)-2\left(h_{E}^{\dagger}\right)_{k j}\left(Y_{E}\right)_{i l} \operatorname{Tr}\left(\Lambda_{E^{l}}^{\dagger} h_{E^{k}}\right) \\
& -2\left(Y_{E}^{\dagger}\right)_{k j}\left(h_{E}\right)_{i l} \operatorname{Tr}\left(h_{E^{l}}^{\dagger} \Lambda_{E^{k}}\right)-2\left(Y_{E}^{\dagger}\right)_{k j}\left(Y_{E}\right)_{i l} \operatorname{Tr}\left(h_{E^{l}}^{\dagger} h_{E^{k}}\right) \\
& -4\left(\Lambda_{E^{l}} \Lambda_{E^{k}}^{\dagger}\right)_{i j}\left(h_{E}^{\dagger} h_{E}\right)_{l k}-4\left(h_{E^{l}} \Lambda_{E^{k}}^{\dagger}\right)_{i j}\left(h_{E}^{\dagger} Y_{E}\right)_{l k}-4\left(\Lambda_{E^{l}} h_{E^{k}}^{\dagger}\right)_{i j}\left(Y_{E}^{\dagger} h_{E}\right)_{l k} \\
& -4\left(h_{E^{l}} h_{E^{k}}^{\dagger}\right)_{i j} \tilde{E}_{l k}-2\left(h_{E^{l}} \Lambda_{E^{k}}^{\dagger}\right)_{i j} \operatorname{Tr}\left(h_{E^{l}}^{\dagger} \Lambda_{E^{k}}\right)-2\left(h_{E^{l}} h_{E^{k}}^{\dagger}\right)_{i j} \operatorname{Tr}\left(\Lambda_{E^{l}}^{\dagger} \Lambda_{E^{k}}\right) \\
& -2\left(\Lambda_{E^{l}} h_{E^{k}}^{\dagger}\right)_{i j} \operatorname{Tr}\left(\Lambda_{E^{l}}^{\dagger} h_{E^{k}}\right)-2\left(\Lambda_{E^{l}} \Lambda_{E^{k}}^{\dagger}\right)_{i j} \operatorname{Tr}\left(h_{E^{l}}^{\dagger} h_{E^{k}}\right) \\
& -2\left(h_{E}\right)_{i k}\left(3 h_{E^{k}}^{\dagger} \Lambda_{D^{p}} Y_{D}^{*}+3 \Lambda_{E^{k}}^{\dagger} \Lambda_{D^{p}} h_{D}^{*}+h_{E^{k}}^{\dagger} \Lambda_{E^{p}} Y_{E}^{*}+\Lambda_{E^{k}}^{\dagger} \Lambda_{E^{p}} h_{E}^{*}\right)_{j p} \\
& -2\left(Y_{E}\right)_{i k}\left(3 h_{E^{k}}^{\dagger} h_{D^{p}} Y_{D}^{*}+3 \Lambda_{E^{k}}^{\dagger} h_{D^{p}} h_{D}^{*}+h_{E^{k}}^{\dagger} h_{E^{p}} Y_{E}^{*}+\Lambda_{E^{k}}^{\dagger} h_{E^{p}} h_{E}^{*}\right)_{j p} \\
& +2\left(h_{E}^{*}\right)_{j k}\left(3 \Lambda_{E^{k}} \Lambda_{D^{p}}^{*} h_{D}+3 h_{E^{k}} \Lambda_{D^{p}}^{*} Y_{D}+\Lambda_{E^{k}} \Lambda_{E^{p}}^{*} h_{E}+h_{E^{k}} \Lambda_{E^{p}}^{*} Y_{E}\right)_{i p} \\
& +2\left(Y_{E}^{*}\right)_{j k}\left(3 \Lambda_{E^{k}} h_{D^{p}}^{*} h_{D}+3 h_{E^{k}} h_{D^{p}}^{*} Y_{D}+\Lambda_{E^{k}} h_{E^{p}}^{*} h_{E}+h_{E^{k}} h_{E^{p}}^{*} Y_{E}\right)_{i p} \\
& -2\left(h_{E^{k}}^{\dagger} h_{E} Y_{E^{\dagger}}^{\dagger} \Lambda_{E^{k}}+h_{E^{k}}^{\dagger} E h_{E^{k}}+\Lambda_{E^{k}}^{\dagger} h_{E} h_{E^{\dagger}}^{\dagger} \Lambda_{E^{k}}+\Lambda_{E^{k}}^{\dagger} Y_{E} h_{E}^{\dagger} h_{E^{k}}\right)_{j i} \\
& -6\left(h_{E^{k}}^{\dagger} h_{D^{p}} \Lambda_{D^{p}}^{\dagger} \Lambda_{E^{k}}+h_{E^{k}}^{\dagger} \Lambda_{D^{p}} \Lambda_{D^{p}}^{\dagger} h_{E^{k}}+\Lambda_{E^{k}}^{\dagger} h_{D^{p}} h_{D^{p}}^{\dagger} \Lambda_{E^{k}}+\Lambda_{E^{k}}^{\dagger} \Lambda_{D^{p}} h_{D^{p}}^{\dagger} h_{E^{k}}\right)_{j i} \\
& -2\left(h_{E^{k}}^{\dagger} h_{E^{p}} \Lambda_{E^{p}}^{\dagger} \Lambda_{E^{k}}+h_{E^{k}}^{\dagger} \Lambda_{E^{p}} \Lambda_{E^{p}}^{\dagger} h_{E^{k}}+\Lambda_{E^{k}}^{\dagger} h_{E^{p}} h_{E^{p}}^{\dagger} \Lambda_{E^{k}}+\Lambda_{E^{k}}^{\dagger} \Lambda_{E^{p}} h_{E^{p}}^{\dagger} h_{E^{k}}\right)_{j i} \\
& -12\left(h_{D^{l}} h_{D^{k}}^{\dagger}\right)_{i j}\left[\tilde{D}_{l k}+\operatorname{Tr}\left(\Lambda_{D^{l}}^{\dagger} \Lambda_{D^{k}}\right)+\left(\Lambda_{U^{q}} \Lambda_{U^{q}}^{\dagger}\right)_{k l}\right] \\
& -12\left(\Lambda_{D^{l}} h_{D^{k}}^{\dagger}\right)_{i j}\left[\left(Y_{D}^{\dagger} h_{D}\right)_{l k}+\operatorname{Tr}\left(\Lambda_{D^{l}}^{\dagger} h_{D^{k}}\right)+\left(h_{U^{q}} \Lambda_{U^{q}}^{\dagger}\right)_{k l}\right] \\
& -12\left(\Lambda_{D^{l}} \Lambda_{D^{k}}^{\dagger}\right)_{i j}\left[\left(h_{D}^{\dagger} h_{D}\right)_{l k}+\operatorname{Tr}\left(h_{D^{l}}^{\dagger} h_{D^{k}}\right)+\left(h_{U^{q}} h_{U^{q}}^{\dagger}\right)_{k l}\right] \\
& -12\left(h_{D^{l}} \Lambda_{D^{k}}^{\dagger}\right)_{i j}\left[\left(h_{D}^{\dagger} Y_{D}\right)_{l k}+\operatorname{Tr}\left(h_{D^{l}}^{\dagger} \Lambda_{D^{k}}\right)+\left(\Lambda_{U^{q}} h_{U^{q}}^{\dagger}\right)_{k l}\right] \\
& -6\left(h_{D^{k}}^{*} h_{D} Y_{D^{\dagger}}^{\dagger} \Lambda_{D^{k}}^{t}+h_{D^{k}}^{*} h_{U} Y_{U}^{\dagger} \Lambda_{D^{k}}^{t}+h_{D^{k}}^{*} Y_{D} Y_{D^{\dagger}}^{\dagger} h_{D^{k}}^{t}+h_{D^{k}}^{*} Y_{U} Y_{U^{\prime}}^{\dagger} h_{D^{k}}^{t}\right)_{j i} \\
& -6\left(\Lambda_{D^{k}}^{*} h_{D} h_{D^{\dagger}}^{\dagger} \Lambda_{D^{k}}^{t}+\Lambda_{D^{k}}^{*} Y_{D} h_{D^{\dagger}}^{\dagger} h_{D^{k}}^{t}+\Lambda_{D^{k}}^{*} h_{U} h_{U}^{\dagger} \Lambda_{D^{k}}^{t}+\Lambda_{D^{k}}^{*} Y_{U} h_{U}^{\dagger} h_{D^{k}}^{t}\right)_{j i} \\
& -6\left(h_{D^{k}} h_{D^{p}}^{\dagger} \Lambda_{D^{p}} \Lambda_{D^{k}}^{\dagger}+\Lambda_{D^{k}} h_{D^{p}}^{\dagger} h_{D^{p}} \Lambda_{D^{k}}^{\dagger}+\Lambda_{D^{k}} \Lambda_{D^{p}}^{\dagger} h_{D^{p}} h_{D^{k}}^{\dagger}+h_{D^{k}} \Lambda_{D^{p}}^{\dagger} \Lambda_{D^{p}} h_{D^{k}}^{\dagger}\right)_{i j} \\
& +\left(16 g_{3}^{2}-\frac{2}{5} g_{1}^{2}\right)\left[\left(m_{\tilde{L}}^{2} \Lambda_{D^{q}} \Lambda_{D^{q}}^{\dagger}\right)_{i j}+2\left(\Lambda_{D^{q}} m_{\tilde{Q}}^{2} \Lambda_{D^{q}}^{t}\right)_{i j}+2\left(m_{\tilde{D}}^{2}\right)_{l q}\left(\Lambda_{D^{l}} \Lambda_{D^{q}}^{\dagger}\right)_{i j}\right. \\
& \left.-\left(Y_{D}^{t} \Lambda_{D^{q}}^{\dagger}\right)_{q j} m_{\tilde{L}_{i} H_{1}}^{2}\right]-4\left(E Y_{E}\right)_{i k}\left(\Lambda_{E^{k}}^{\dagger}\right)_{q j} m_{\tilde{L}_{q} H_{1}}^{2}+3\left(\Lambda_{E^{k}}^{*} E Y_{E}\right)_{j k} m_{\tilde{L}_{i} H_{1}}^{2} \\
& +\frac{6}{5} g_{1}^{2}\left(\Lambda_{E^{k}}\right)_{i l}\left(Y_{E}^{\dagger}\right)_{k j} m_{H_{1} \tilde{L}_{l}}^{2}+\frac{6}{5} g_{1}^{2}\left[\left(m_{\tilde{L}^{2}}^{2} \Lambda_{E^{q}} \Lambda_{E^{q}}^{\dagger}\right)_{i j}+2\left(\Lambda_{E^{q}} m_{\tilde{L}}^{2 t} \Lambda_{E^{q}}^{\dagger}\right)_{i j}\right.
\end{aligned}
$$




$$
\begin{aligned}
& \left.+2\left(m_{\tilde{E}}^{2}\right)_{l q}\left(\Lambda_{E^{l}} \Lambda_{E^{q}}^{\dagger}\right)_{i j}+\left(Y_{E}\right)_{i q}\left(\Lambda_{E^{q}}^{\dagger}\right)_{k j} m_{\tilde{L}_{k} H_{1}}^{2}-\left(Y_{E}^{t} \Lambda_{E^{q}}^{\dagger}\right)_{q j} m_{\tilde{L}_{i} H_{1}}^{2}\right] \\
& -4\left(\Lambda_{E^{k}}\right)_{i l}\left(Y_{E}^{\dagger} E\right)_{k j} m_{H_{1} \tilde{L}_{l}}^{2}-3\left(Y_{E}^{\dagger}\right)_{k j}\left(E \Lambda_{E^{k}}\right)_{i l} m_{H_{1} \tilde{L}_{l}}^{2} \\
& -\left(Y_{E}^{\dagger}\right)_{k j}\left(m_{\tilde{L}}^{2} Y_{E}\right)_{i l} \operatorname{Tr}\left(\Lambda_{E^{l}}^{\dagger} \Lambda_{E^{k}}\right)-2\left(Y_{E}^{\dagger}\right)_{k j}\left(Y_{E} m_{\tilde{E}}^{2}\right)_{i l} \operatorname{Tr}\left(\Lambda_{E^{l}}^{\dagger} \Lambda_{E^{k}}\right) \\
& -2 m_{H_{1}}^{2}\left(Y_{E}^{\dagger}\right)_{k j}\left(Y_{E}\right)_{i l} \operatorname{Tr}\left(\Lambda_{E^{l}}^{\dagger} \Lambda_{E^{k}}\right)-\left(Y_{E}^{\dagger}\right)_{k j}\left(\Lambda_{E^{l}}\right)_{i q} \operatorname{Tr}\left(\Lambda_{E^{l}}^{\dagger} \Lambda_{E^{k}}\right) m_{H_{1} \tilde{L}_{q}}^{2} \\
& -2\left(m_{\tilde{E}}^{2} Y_{E}^{\dagger}\right)_{q j}\left(Y_{E}\right)_{i l} \operatorname{Tr}\left(\Lambda_{E^{l}}^{\dagger} \Lambda_{E^{q}}\right)-2\left(\Lambda_{E^{l}}^{\dagger} E\right)_{m j}\left(Y_{E}\right)_{i l} m_{\tilde{L}_{m} H_{1}}^{2} \\
& -2\left(m_{\tilde{L}^{2}}^{2} \Lambda_{E^{l}} \Lambda_{E^{k}}^{\dagger}\right)_{i j} \tilde{E}_{l k}-3\left(\Lambda_{D^{k}}^{*} T \Lambda_{D^{k}}^{t}\left(m_{\tilde{L}}^{2}\right)^{t}\right)_{j i} \\
& -4\left(\Lambda_{E^{l}} m_{\tilde{L}}^{2 t} \Lambda_{E^{k}}^{\dagger}\right)_{i j} \tilde{E}_{l k}-4\left(m_{\tilde{E}}^{2} \tilde{E}\right)_{q k}\left(\Lambda_{E^{q}} \Lambda_{E^{k}}^{\dagger}\right)_{i j} \\
& -4\left(\Lambda_{E^{l}} \Lambda_{E^{k}}^{\dagger}\right)_{i j}\left(Y_{E}^{\dagger} m_{\tilde{L}}^{2} Y_{E}\right)_{l k}-4\left(\Lambda_{E^{l}} \Lambda_{E^{k}}^{\dagger}\right)_{i j}\left[\tilde{E} m_{\tilde{E}}^{2}\right]_{l k} \\
& -4 m_{H_{1}}^{2}\left(\Lambda_{E^{l}} \Lambda_{E^{k}}^{\dagger}\right)_{i j} \tilde{E}_{l k}-2\left(\Lambda_{E^{l}} \Lambda_{E^{k}}^{\dagger}\right)_{i j}\left(Y_{E}^{\dagger} \Lambda_{E^{k}}\right)_{l q} m_{H_{1} \tilde{L}_{q}}^{2} \\
& -\left(m_{\tilde{L}^{2}}^{2} \Lambda_{E^{l}} \Lambda_{E^{k}}^{\dagger}\right)_{i j} \operatorname{Tr}\left(\Lambda_{E^{l}}^{\dagger} \Lambda_{E^{k}}\right)-2\left(\Lambda_{E^{l}} m_{\tilde{L}}^{2 t} \Lambda_{E^{k}}^{\dagger}\right)_{i j} \operatorname{Tr}\left(\Lambda_{E^{l}}^{\dagger} \Lambda_{E^{k}}\right) \\
& -2\left(m_{\tilde{E}}^{2}\right)_{q l}\left(\Lambda_{E^{q}} \Lambda_{E^{k}}^{\dagger}\right)_{i j} \operatorname{Tr}\left(\Lambda_{E^{l}}^{\dagger} \Lambda_{E^{k}}\right)-\operatorname{Tr}\left(\Lambda_{E^{l}}^{\dagger} \Lambda_{E^{k}}\right)\left(Y_{E}\right)_{i l}\left(\Lambda_{E^{k}}^{\dagger}\right)_{q j} m_{\tilde{L}_{q} H_{1}}^{2} \\
& +\operatorname{Tr}\left(\Lambda_{E^{l}}^{\dagger} \Lambda_{E^{k}}\right)\left(Y_{E}^{t} \Lambda_{E^{k}}^{\dagger}\right)_{l j} m_{\tilde{L}_{i} H_{1}}^{2}-4\left(\Lambda_{E^{l}} \Lambda_{E^{k}}^{\dagger}\right)_{i j} \operatorname{Tr}\left(\Lambda_{E^{l}}^{\dagger} m_{\tilde{L}^{2}}^{2} \Lambda_{E^{k}}\right) \\
& -2\left(m_{\tilde{E}}^{2}\right)_{q k}\left(\Lambda_{E^{l}} \Lambda_{E^{k}}^{\dagger}\right)_{i j} \operatorname{Tr}\left(\Lambda_{E^{l}}^{\dagger} \Lambda_{E^{q}}\right)-4\left(\Lambda_{E^{l}} \Lambda_{E^{k}}^{\dagger}\right)_{i j}\left(\Lambda_{E^{l}}^{\dagger} Y_{E}\right)_{q k} m_{\tilde{L}_{q} H_{1}}^{2} \\
& -\operatorname{Tr}(2 E+6 D)\left(\Lambda_{E^{k}}\right)_{i l}\left(Y_{E}^{\dagger}\right)_{k j} m_{H_{1} \tilde{L}_{l}}^{2}+3\left(\Lambda_{D^{k}}^{*} Y_{D}\right)_{j l}\left(\Lambda_{D^{l}}^{*} \Lambda_{D^{k}}^{t}\right)_{q i} m_{\tilde{L}_{q} H_{1}}^{2} \\
& -E_{i j}\left(Y_{E}^{\dagger} \Lambda_{E^{k}}\right)_{k l} m_{H_{1} \tilde{L}_{l}}^{2}+6 E_{i j}\left(Y_{D}^{\dagger} \Lambda_{D^{k}}^{t}\right)_{k l} m_{H_{1} \tilde{L}_{l}}^{2} \\
& -3\left(m_{\tilde{L}}^{2} Y_{E}\right)_{i k}\left(\Lambda_{E^{k}}^{\dagger} \Lambda_{D^{p}} Y_{D}^{*}\right)_{j p}-\left(m_{\tilde{L}}^{2} Y_{E}\right)_{i k}\left(\Lambda_{E^{k}}^{\dagger} \Lambda_{E^{p}} Y_{E}^{*}\right)_{j p} \\
& -m_{H_{1}}^{2}\left(Y_{E}\right)_{i k}\left[6 \Lambda_{E^{k}}^{\dagger} \Lambda_{D^{p}} Y_{D}^{*}+2 \Lambda_{E^{k}}^{\dagger} \Lambda_{E^{p}} Y_{E}^{*}\right]_{j p} \\
& -6\left(Y_{E} m_{\tilde{E}}^{2}\right)_{i k}\left(\Lambda_{E^{k}}^{\dagger} \Lambda_{D^{p}} Y_{D}^{*}\right)_{j p}-2\left(Y_{E} m_{\tilde{E}}^{2}\right)_{i k}\left(\Lambda_{E^{k}}^{\dagger} \Lambda_{E^{p}} Y_{E}^{*}\right)_{j p} \\
& -3\left(\Lambda_{E^{k}}\right)_{i l} m_{H_{1} \tilde{L}_{l}}^{2}\left(\Lambda_{E^{k}}^{\dagger} \Lambda_{D^{p}} Y_{D}^{*}\right)_{j p}-\left(\Lambda_{E^{k}}\right)_{i l} m_{H_{1} \tilde{L}_{l}}^{2}\left(\Lambda_{E^{k}}^{\dagger} \Lambda_{E^{p}} Y_{E}^{*}\right)_{j p} \\
& -6\left(Y_{E}\right)_{i k}\left(\Lambda_{E^{k}}^{\dagger} m_{\tilde{L}^{2}}^{2} \Lambda_{D^{p}} Y_{D}^{*}\right)_{j p}-6\left(Y_{E}\right)_{i k}\left(\Lambda_{E^{k}}^{\dagger} \Lambda_{D^{p}} m_{\tilde{Q}}^{2 t} Y_{D}^{*}\right)_{j p} \\
& -3\left(Y_{E}\right)_{i k}\left(\Lambda_{E^{k}}^{\dagger} \Lambda_{D^{l}} Y_{D}^{*} m_{\tilde{D}}^{2}\right)_{j l}^{t}+6\left(Y_{E}\right)_{i k}\left(\Lambda_{E^{k}}^{\dagger}\right)_{j l} \operatorname{Tr}(D) m_{\tilde{L}_{l} H_{1}}^{2} \\
& -2\left(Y_{E}\right)_{i k}\left(\Lambda_{E^{k}}^{\dagger} m_{\tilde{L}}^{2} \Lambda_{E^{p}} Y_{E}^{*}\right)_{j p}-2\left(Y_{E}\right)_{i k}\left(\Lambda_{E^{k}}^{\dagger} \Lambda_{E^{p}} m_{\tilde{L}}^{2}{ }^{t} Y_{E}^{*}\right)_{j p} \\
& -\left(Y_{E}\right)_{i k}\left(m_{\tilde{E}}^{2} Y_{E}^{\dagger} \Lambda_{E^{l}}^{t} \Lambda_{E^{k}}^{*}\right)_{l j}+2\left(Y_{E}\right)_{i k}\left(\Lambda_{E^{k}}^{\dagger}\right)_{j l} \operatorname{Tr}(E) m_{\tilde{L}_{l} H_{1}}^{2} \\
& +6 m_{H_{1}}^{2}\left(Y_{E}^{\dagger}\right)_{k j}\left(\Lambda_{E^{k}} \Lambda_{D^{p}}^{*} Y_{D}\right)_{i p}-3\left(Y_{E}^{\dagger}\right)_{k j}\left(\Lambda_{E^{k}} \Lambda_{D^{p}}^{*} \Lambda_{D^{p}}^{t}\right)_{i l} m_{H_{1} \tilde{L}_{l}}^{2} \\
& +2\left(Y_{E}^{\dagger}\right)_{k j}\left(\Lambda_{E^{k}} \Lambda_{E p}^{*} m_{\tilde{L}}^{2} Y_{E}+\Lambda_{E^{k}} \Lambda_{E^{p}}^{*} Y_{E} m_{\tilde{E}}^{2}\right)_{i p}+2 m_{H_{1}}^{2}\left(Y_{E}^{\dagger}\right)_{k j}\left(\Lambda_{E^{k}} \Lambda_{E^{p}}^{*} Y_{E}\right)_{i p} \\
& +m_{H_{1} \tilde{L}_{l}}^{2}\left(\Lambda_{E^{k}} \Lambda_{E^{p}}^{*} \Lambda_{E^{p}}\right)_{i l}\left(Y_{E}^{\dagger}\right)_{k j}+3\left(Y_{E}^{\dagger}\right)_{k j}\left(m_{\tilde{L}}^{2} \Lambda_{E^{k}} \Lambda_{D^{p}}^{*} Y_{D}+2 \Lambda_{E^{k}} m_{\tilde{L}}^{2 t} \Lambda_{D^{p}}^{*} Y_{D}\right)_{i p} \\
& +6\left(m_{\tilde{E}}^{2} Y_{E}^{\dagger}\right)_{l j}\left(\Lambda_{E^{l}} \Lambda_{D^{p}}^{*} Y_{D}\right)_{i p}+6 E_{i j}\left(\Lambda_{D^{p}}^{*} Y_{D}\right)_{k p} m_{\tilde{L}_{k} H_{1}}^{2} \\
& -3\left(Y_{D}^{t} \Lambda_{D^{p}}^{\dagger} E\right)_{j p} m_{\tilde{L}_{i} H_{1}}^{2}+\left(Y_{E}^{\dagger}\right)_{k j}\left(m_{\tilde{L}}^{2} \Lambda_{E^{k}} \Lambda_{E^{p}}^{*} Y_{E}+2 \Lambda_{E^{k}} m_{\tilde{L}}^{2 t} \Lambda_{E^{p}}^{*} Y_{E}\right)_{i p} \\
& +2\left(m_{\tilde{E}}^{2} Y_{E}^{\dagger}\right)_{l j}\left(\Lambda_{E^{l}} \Lambda_{E^{p}}^{*} Y_{E}\right)_{i p}+2 E_{i j}\left(\Lambda_{E^{p}}^{*} Y_{E}\right)_{k p} m_{\tilde{L}_{k} H_{1}}^{2} \\
& -\left(Y_{E}^{t} \Lambda_{E^{p}}^{\dagger} E\right)_{p j} m_{\tilde{L}_{i} H_{1}}^{2}-2\left(\Lambda_{E^{k}}^{\dagger} m_{\tilde{L}}^{2} E \Lambda_{E^{k}}\right)_{j i} \\
& -2\left(\Lambda_{E^{k}}^{\dagger} Y_{E} m_{\tilde{E}}^{2} Y_{E}^{\dagger} \Lambda_{E^{k}}\right)_{j i}-\left(2 \Lambda_{E^{k}}^{\dagger} E m_{\tilde{L}^{2}}^{2} \Lambda_{E^{k}}+\Lambda_{E^{k}}^{\dagger} E \Lambda_{E^{k}} m_{\tilde{L}}^{2 t}\right)_{j i} \\
& -\left(\Lambda_{E^{k}}^{\dagger} \Lambda_{E^{m}}\right)_{j q}\left(Y_{E}^{\dagger} \Lambda_{E^{k}}\right)_{m i} m_{H_{1} \tilde{L}_{q}}^{2}-2 m_{H_{1}}^{2}\left(\Lambda_{E^{k}}^{\dagger} E \Lambda_{E^{k}}\right)_{j i} \\
& -2\left(m_{\widetilde{E}}^{2}\right)_{l k}\left(\Lambda_{E^{k}}^{\dagger} E \Lambda_{E^{l}}\right)_{j i}-\left(\Lambda_{E^{k}}^{\dagger} E Y_{E}\right)_{j k} m_{\tilde{L}_{i} H_{1}}^{2}+6\left(Y_{E}^{\dagger}\right)_{k j}\left(\Lambda_{E^{k}} \Lambda_{D^{p}}^{*} m_{\widetilde{Q}^{2}}^{2} Y_{D}\right.
\end{aligned}
$$




$$
\begin{aligned}
& \left.+\Lambda_{E^{k}} \Lambda_{D^{p}}^{*} Y_{D} m_{\tilde{D}}^{2}\right)_{i p}-6\left(\Lambda_{E^{k}}^{\dagger} m_{\tilde{L}}^{2} \Lambda_{D^{p}} \Lambda_{D^{p}}^{\dagger} \Lambda_{E^{k}}+\Lambda_{E^{k}}^{\dagger} \Lambda_{D^{p}} m_{\tilde{Q}}^{2 t} \Lambda_{D^{p}}^{\dagger} \Lambda_{E^{k}}\right)_{j i} \\
& -6\left(m_{\tilde{D}}^{2}\right)_{l p}\left(\Lambda_{E^{k}}^{\dagger} \Lambda_{D^{l}} \Lambda_{D^{p}}^{\dagger} \Lambda_{E^{k}}\right)_{j i}+3\left(Y_{D}^{t} \Lambda_{D^{p}}^{\dagger} \Lambda_{E^{k}}\right)_{p i}\left(\Lambda_{E^{k}}^{\dagger}\right)_{j l} m_{\tilde{L}_{l} H_{1}}^{2} \\
& +3\left(2 \Lambda_{E^{k}}^{\dagger} \Lambda_{D^{p}} \Lambda_{D^{p}}^{\dagger} m_{\tilde{L}^{2}}^{2} \Lambda_{E^{k}}+\Lambda_{E^{k}}^{\dagger} \Lambda_{D^{p}} \Lambda_{D^{p}}^{\dagger} \Lambda_{E^{k}} m_{\tilde{L}^{2}}^{2 t}\right)_{j i} \\
& -6\left(m_{\tilde{E}}^{2}\right)_{l k}\left(\Lambda_{E^{k}}^{\dagger} \Lambda_{D^{p}} \Lambda_{D^{p}}^{\dagger} \Lambda_{E^{l}}\right)_{j i}-\left(\Lambda_{E^{k}}^{\dagger} \Lambda_{E^{p}} \Lambda_{E^{p}}^{\dagger} Y_{E}\right)_{j k} m_{\tilde{L}_{i} H_{1}}^{2} \\
& -3\left(\Lambda_{E^{k}}^{\dagger} \Lambda_{D^{p}} \Lambda_{D^{p}}^{\dagger} Y_{E}\right)_{j k} m_{\tilde{L}_{i} H_{1}}^{2}+3\left(\Lambda_{E^{k}}^{\dagger} \Lambda_{D^{p}} \Lambda_{D^{p}}^{\dagger}\right)_{j q}\left(Y_{E}\right)_{i k} m_{\tilde{L}_{q} H_{1}}^{2} \\
& +2\left(\Lambda_{E^{k}}^{\dagger} \Lambda_{E^{p}} \Lambda_{E^{p}}^{\dagger}\right)_{j q}\left(Y_{E}\right)_{i k} m_{\tilde{L}_{q} H_{1}}^{2}-2\left(m_{\tilde{E}}^{2}\right)_{l k}\left(\Lambda_{E^{k}}^{\dagger} \Lambda_{E^{p}} \Lambda_{E^{p}}^{\dagger} \Lambda_{E^{l}}\right)_{j i} \\
& -\left(2 \Lambda_{E^{k}}^{\dagger} \Lambda_{E^{p}} \Lambda_{E^{p}}^{\dagger} m_{\tilde{L}}^{2} \Lambda_{E^{k}}+\Lambda_{E^{k}}^{\dagger} \Lambda_{E^{p}} \Lambda_{E^{p}}^{\dagger} \Lambda_{E^{k}} m_{\tilde{L}}^{2 t}\right)_{j i} \\
& -2\left(\Lambda_{E^{k}}^{\dagger} m_{\tilde{L}^{2}}^{2} \Lambda_{E^{p}} \Lambda_{E^{p}}^{\dagger} \Lambda_{E^{k}}+\Lambda_{E^{k}}^{\dagger} \Lambda_{E^{p}} m_{\tilde{L}}^{2 t} \Lambda_{E^{p}}^{\dagger} \Lambda_{E^{k}}\right)_{j i} \\
& -2\left(m_{\tilde{E}}^{2}\right)_{l p}\left(\Lambda_{E^{k}}^{\dagger} \Lambda_{E^{l}} \Lambda_{E^{p}}^{\dagger} \Lambda_{E^{k}}\right)_{j i}-\left(\Lambda_{E^{k}}^{\dagger} Y_{E}\right)_{j p}\left(\Lambda_{E^{p}}^{\dagger} \Lambda_{E^{k}}\right)_{q i} m_{\tilde{L}_{q} H_{1}}^{2} \\
& +\left(Y_{E}^{t} \Lambda_{E^{p}}^{\dagger} \Lambda_{E^{k}}\right)_{p i}\left(\Lambda_{E^{k}}^{\dagger}\right)_{j l} m_{\tilde{L}_{l} H_{1}}^{2}+6\left(\Lambda_{U^{q}} \Lambda_{U^{q}}^{\dagger} Y_{D}^{t} \Lambda_{D^{k}}^{\dagger}\right)_{k j} m_{\tilde{L}_{i} H_{1}}^{2} \\
& -12\left(\Lambda_{D^{l}} m_{\tilde{Q}}^{2 t} \Lambda_{D^{k}}^{\dagger}\right)_{i j}\left[(\tilde{D})_{l k}+\operatorname{Tr}\left(\Lambda_{D^{l}}^{\dagger} \Lambda_{D^{k}}\right)+\left(\Lambda_{U^{q}} \Lambda_{U^{q}}^{\dagger}\right)_{k l}\right] \\
& -6\left(m_{\tilde{L}}^{2} \Lambda_{D^{l}} \Lambda_{D^{k}}^{\dagger}\right)_{i j} \tilde{D}_{l k}-6\left(m_{\tilde{L}^{2}}^{2} \Lambda_{D^{l}} \Lambda_{D^{k}}^{\dagger}\right)_{i j} \operatorname{Tr}\left(\Lambda_{D^{l}}^{\dagger} \Lambda_{D^{k}}\right) \\
& -6\left(m_{\tilde{L}}^{2} \Lambda_{D^{l}} \Lambda_{D^{k}}^{\dagger}\right)_{i j}\left(\Lambda_{U^{q}} \Lambda_{U^{q}}^{\dagger}\right)_{k l}+9\left(\Lambda_{D^{k}}^{*} D Y_{D}\right)_{j k} m_{\tilde{L}_{i} H_{1}}^{2} . \\
& +6\left(\Lambda_{D^{k}}^{*} Y_{D}\right)_{j l} \operatorname{Tr}\left(\Lambda_{D^{l}}^{\dagger} \Lambda_{D^{k}}\right) m_{\tilde{L}_{i} H_{1}}^{2}-12\left(\Lambda_{D^{l}} \Lambda_{D^{k}}^{\dagger}\right)_{i j}\left(Y_{D^{\prime}}^{\dagger} m_{\tilde{Q}}^{2} Y_{D}\right)_{l k} \\
& -12\left(\Lambda_{D^{l}} \Lambda_{D^{k}}^{\dagger}\right)_{i j}\left(\tilde{D} m_{\tilde{D}}^{2}\right)_{l k}-12 m_{H_{1}}^{2}\left(\Lambda_{D^{l}} \Lambda_{D^{k}}^{\dagger}\right)_{i j}(\tilde{D})_{l k} \\
& +6 m_{H_{1} \tilde{L}_{q}}^{2}\left(Y_{D^{\dagger}}^{\dagger} \Lambda_{D^{k}}^{t}\right)_{l q}\left(\Lambda_{D^{l}} \Lambda_{D^{k}}^{\dagger}\right)_{i j}-12\left(\Lambda_{D^{l}} \Lambda_{D^{k}}^{\dagger}\right)_{i j}\left[\operatorname{Tr}\left(\Lambda_{D^{l}}^{\dagger} m_{\tilde{L}}^{2} \Lambda_{D^{k}}\right)\right. \\
& \left.+\operatorname{Tr}\left(\Lambda_{D^{l}}^{\dagger} \Lambda_{D^{k}} m_{\tilde{Q}}^{2}{ }^{t}\right)\right]-4\left(Y_{E}^{\dagger}\right)_{k j}\left(Y_{E}\right)_{i l} \operatorname{Tr}\left(\Lambda_{E^{l}}^{\dagger} m_{\tilde{L}}^{2} \Lambda_{E^{k}}\right) \\
& -12\left(m_{\tilde{D}}^{2}\right)_{q k}\left(\Lambda_{D^{l}} \Lambda_{D^{k}}^{\dagger}\right)_{i j} \operatorname{Tr}\left(\Lambda_{D^{l}}^{\dagger} \Lambda_{D^{q}}\right)+6\left(\Lambda_{D^{l}} \Lambda_{D^{k}}^{\dagger}\right)_{i j}\left(Y_{D}^{t} \Lambda_{D^{l}}^{\dagger}\right)_{k q} m_{\tilde{L}_{q} H_{1}}^{2} \\
& -12\left(\Lambda_{D^{l}} \Lambda_{D^{k}}^{\dagger}\right)_{i j}\left(\Lambda_{U^{q}} m_{\tilde{D}^{2}}^{2} \Lambda_{U^{q}}^{\dagger}\right)_{k l}-12\left(\Lambda_{D^{l}} \Lambda_{D^{k}}^{\dagger}\right)_{i j}\left(m_{\tilde{D}}^{2}{ }^{t} \Lambda_{U^{q}} \Lambda_{U^{q}}^{\dagger}\right)_{k l} \\
& -12\left(\Lambda_{D^{l}} \Lambda_{D^{k}}^{\dagger}\right)_{i j}\left(\Lambda_{U^{p}} \Lambda_{U^{q}}^{\dagger}\right)_{k l}\left(m_{\tilde{U}}^{2}\right)_{p q}-6\left(\Lambda_{D^{k}}^{*} m_{\tilde{Q}}^{2} D \Lambda_{D^{k}}^{t}\right)_{j i} \\
& -6\left(\Lambda_{D^{k}}^{*} Y_{D} m_{\tilde{D}}^{2} Y_{D}^{\dagger} \Lambda_{D^{k}}^{t}\right)_{j i}-6 m_{H_{1}}^{2}\left(\Lambda_{D^{k}}^{*} D \Lambda_{D^{k}}^{t}\right)_{j i} \\
& +3\left(\Lambda_{D^{k}}^{*} \Lambda_{D^{m}}^{t}\right)_{j q}\left(Y_{D^{\dagger}}^{\dagger} \Lambda_{D^{k}}^{t}\right)_{m i} m_{H_{1} \tilde{L}_{q}}^{2}-3\left(m_{\tilde{L}^{2}}^{2} \Lambda_{D^{k}} D^{t} \Lambda_{D^{k}}^{\dagger}\right)_{i j} \\
& -6\left(\Lambda_{D^{k}} m_{\tilde{Q}}^{2 t} D^{t} \Lambda_{D^{k}}^{\dagger}\right)_{i j}-6\left(m_{\tilde{D}}^{2}\right)_{l k}\left(\Lambda_{D^{l}} D^{t} \Lambda_{D^{k}}^{\dagger}\right)_{i j}-6\left(m_{\tilde{D}}^{2}\right)_{l k}\left(\Lambda_{D^{k}}^{*} T \Lambda_{D^{l}}^{t}\right)_{j i} \\
& +3\left(\Lambda_{D^{k}}^{*} T Y_{D}\right)_{j k} m_{\tilde{L}_{i} H_{1}}^{2}-6\left(\Lambda_{D^{k}}^{*} m_{\tilde{Q}^{2}}^{2} T \Lambda_{D^{k}}^{t}\right)_{j i}-6\left(\Lambda_{D^{k}}^{*} Y_{U} m_{\tilde{U}^{2}}^{2} Y_{U}^{\dagger} \Lambda_{D^{k}}^{t}\right)_{j i} \\
& -3\left(m_{\tilde{L}^{2}}^{2} \Lambda_{D^{k}} \Lambda_{D^{p}}^{\dagger} \Lambda_{D^{p}} \Lambda_{D^{k}}^{\dagger}\right)_{i j}-6\left(\Lambda_{D^{k}} m_{\tilde{Q}}^{2}{ }^{t} \Lambda_{D^{p}}^{\dagger} \Lambda_{D^{p}} \Lambda_{D^{k}}^{\dagger}\right)_{i j} \\
& +3\left(Y_{D}^{t} \Lambda_{D^{p}}^{\dagger} \Lambda_{D^{p}} \Lambda_{D^{k}}^{\dagger}\right)_{k j} m_{\tilde{L}_{i} H_{1}}^{2}-6\left(\Lambda_{D^{k}} \Lambda_{D^{p}}^{\dagger} m_{\tilde{L}}^{2} \Lambda_{D^{p}} \Lambda_{D^{k}}^{\dagger}\right)_{i j} \\
& -6\left(m_{\tilde{D}}^{2}\right)_{l p}\left(\Lambda_{D^{k}} \Lambda_{D^{p}}^{\dagger} \Lambda_{D^{l}} \Lambda_{D^{k}}^{\dagger}\right)_{i j}+3\left(Y_{D}^{t} \Lambda_{D^{k}}^{\dagger}\right)_{p j}\left(\Lambda_{D^{k}} \Lambda_{D^{p}}^{\dagger}\right)_{i l} m_{\tilde{L}_{l} H_{1}}^{2} \\
& -12\left(m_{\tilde{D}}^{2}\right)_{p l} \operatorname{Tr}\left(\Lambda_{D^{l}}^{\dagger} \Lambda_{D^{k}}\right)\left(\Lambda_{D^{p}} \Lambda_{D^{k}}^{\dagger}\right)_{i j}-6\left(m_{\tilde{D}}^{2}\right)_{l k}\left(\Lambda_{D^{l}} \Lambda_{D^{p}}^{\dagger} \Lambda_{D^{p}} \Lambda_{D^{k}}^{\dagger}\right)_{i j} \\
& +\left(16 g_{3}^{2}-\frac{2}{5} g_{1}^{2}\right)\left[\left(\Lambda_{D^{q}} \Lambda_{D^{q}}^{\dagger} m_{\tilde{L}}^{2}\right)_{i j}-\left(\Lambda_{D^{q}} Y_{D}^{*}\right)_{i q} m_{H_{1} \tilde{L}_{j}}^{2}\right] \\
& +\frac{6}{5} g_{1}^{2}\left[\left(Y_{E}\right)_{i k}\left(\Lambda_{E^{k}}^{\dagger}\right)_{l j} m_{\tilde{L}_{l} H_{1}}^{2}+\left(\Lambda_{E^{q}} \Lambda_{E^{q}}^{\dagger} m_{\tilde{L}}^{2}\right)_{i j}+\left(\Lambda_{E^{q}}\right)_{i k}\left(Y_{E}^{\dagger}\right)_{q j} m_{H_{1} \tilde{L}_{k}}^{2}\right. \\
& \left.-\left(\Lambda_{E^{q}} Y_{E}^{*}\right)_{i q} m_{H_{1} \tilde{L}_{j}}^{2}\right]-6 m_{H_{2}}^{2}\left(\Lambda_{D^{k}}^{*} T \Lambda_{D^{k}}^{t}\right)_{j i}-6\left(\Lambda_{D^{k}}^{*} T m_{\tilde{Q}^{2}}^{2} \Lambda_{D^{k}}^{t}\right)_{i j} \\
& -2\left(Y_{E}\right)_{i l}\left(\Lambda_{E^{l}}^{\dagger} E\right)_{q j} m_{\tilde{L}_{q} H_{1}}^{2}-\left(Y_{E}^{\dagger} m_{\tilde{L}}^{2}\right)_{k j}\left(Y_{E}\right)_{i l} \operatorname{Tr}\left(\Lambda_{E^{l}}^{\dagger} \Lambda_{E^{k}}\right)
\end{aligned}
$$




$$
\begin{aligned}
& -m_{\tilde{L}_{q} H_{1}}^{2}\left(\Lambda_{E^{k}}^{\dagger}\right)_{q j}\left(Y_{E}\right)_{i l} \operatorname{Tr}\left(\Lambda_{E^{l}}^{\dagger} \Lambda_{E^{k}}\right)+\left(Y_{E}^{\dagger}\right)_{k j}\left(E \Lambda_{E^{k}}^{t}\right)_{i q} m_{H_{1} \tilde{L}_{q}}^{2} \\
& -2 \tilde{E}_{l k}\left(\Lambda_{E^{l}} \Lambda_{E^{k}}^{\dagger} m_{\tilde{L}^{2}}^{2}\right)_{i j}+3\left(Y_{E}^{\dagger} E \Lambda_{E^{l}}^{t}\right)_{l i} m_{H_{1} \tilde{L}_{j}}^{2}-\left(Y_{E^{\prime}}^{\dagger} \Lambda_{E^{p}}^{t} \Lambda_{E^{k}}^{*} m_{\tilde{L}}^{2}\right)_{p j}\left(Y_{E}\right)_{i k} \\
& -\left(\Lambda_{E^{l}} \Lambda_{E^{k}}^{\dagger} m_{\tilde{L}}^{2}\right)_{i j} \operatorname{Tr}\left(\Lambda_{E^{l}}^{\dagger} \Lambda_{E^{k}}\right)-\left(\Lambda_{E^{l}}\right)_{i q}\left(Y_{E}^{\dagger}\right)_{k j} \operatorname{Tr}\left(\Lambda_{E^{l}}^{\dagger} \Lambda_{E^{k}}\right) m_{H_{1} \tilde{L}_{q}}^{2} \\
& +\left(\Lambda_{E^{l}} Y_{E}^{*}\right)_{i k} \operatorname{Tr}\left(\Lambda_{E^{l}}^{\dagger} \Lambda_{E^{k}}\right) m_{H_{1} \tilde{L}_{j}}^{2}-\left(Y_{E}^{\dagger} \Lambda_{E^{k}}\right)_{l q}\left(\Lambda_{E^{l}} \Lambda_{E^{k}}^{\dagger}\right)_{i j} m_{H_{1} \tilde{L}_{q}}^{2} \\
& +\left(\Lambda_{E^{k}} Y_{E}^{*}\right)_{q l}\left(\Lambda_{E^{l}} \Lambda_{E^{k}}^{\dagger}\right)_{i j} m_{H_{1} \tilde{L}_{q}}^{2}-E_{i j}\left(\Lambda_{E^{k}}^{\dagger} Y_{E}\right)_{l k} m_{\tilde{L}_{l} H_{1}}^{2} \\
& -3\left(Y_{E}\right)_{i k}\left(Y_{D}^{\dagger} \Lambda_{D^{p}}^{t} \Lambda_{E^{k}}^{*} m_{\tilde{L}}^{2}\right)_{p j}-3\left(E \Lambda_{D^{p}} Y_{D}^{*}\right)_{i p} m_{H_{1} \tilde{L}_{j}}^{2}-\left(E \Lambda_{E^{p}} Y_{E}^{*}\right)_{i p} m_{H_{1} \tilde{L}_{j}}^{2} \\
& -3\left(Y_{E}\right)_{i k}\left(m_{\tilde{D}}^{2} Y_{D}^{\dagger} \Lambda_{D^{p}}^{t} \Lambda_{E^{k}}^{*}\right)_{p j}+3\left(Y_{E}\right)_{i k}\left(\Lambda_{E^{k}}^{\dagger} \Lambda_{D^{p}} \Lambda_{D^{p}}^{\dagger}\right)_{j l} m_{\tilde{L}_{l} H_{1}}^{2} \\
& -\left(Y_{E}\right)_{i k}\left(m_{\tilde{E}}^{2} Y_{E}^{\dagger} \Lambda_{E^{p}}^{t} \Lambda_{E^{k}}^{*}\right)_{p j}+\left(Y_{E}^{\dagger}\right)_{k j}\left(E \Lambda_{E^{k}}\right)_{q i} m_{H_{1} \tilde{L}_{q}}^{2} \\
& +\left(Y_{E}^{\dagger} m_{\tilde{L}}^{2}\right)_{k j}\left(3 \Lambda_{E^{k}} \Lambda_{D^{p}}^{*} Y_{D}+\Lambda_{E^{k}} \Lambda_{E^{p}}^{*} Y_{E}\right)_{i p}+\left(\Lambda_{E^{k}}^{\dagger}\right)_{l j}\left(3 \Lambda_{E^{k}} \Lambda_{D^{p}}^{*} Y_{D}\right. \\
& \left.+\Lambda_{E^{k}} \Lambda_{E^{p}}^{*} Y_{E}\right)_{i p} m_{\tilde{L}_{l} H_{1}}^{2}+\left(Y_{E}^{\dagger}\right)_{k j}\left(E \Lambda_{E^{k}}^{t}\right)_{l i} m_{H_{1} \tilde{L}_{l}}^{2}-\left(m_{\tilde{L}}^{2} \Lambda_{E^{k}}^{\dagger} E \Lambda_{E^{k}}\right)_{j i} \\
& -\left(\Lambda_{E^{m}}^{\dagger} \Lambda_{E^{k}}\right)_{l i}\left(\Lambda_{E^{k}}^{\dagger} Y_{E}\right)_{j m} m_{\tilde{L}_{l} H_{1}}^{2}-3\left(m_{\tilde{L}}^{2} \Lambda_{E^{k}}^{\dagger} \Lambda_{D^{p}} \Lambda_{D^{p}}^{\dagger} \Lambda_{E^{k}}\right)_{j i} \\
& -3\left(Y_{E}^{\dagger} \Lambda_{D^{p}} \Lambda_{D^{p}}^{\dagger} \Lambda_{E^{k}}\right)_{k i} m_{H_{1} \tilde{L}_{j}}^{2}+3\left(Y_{E}^{\dagger}\right)_{k j}\left(\Lambda_{D^{p}} \Lambda_{D^{p}}^{\dagger} \Lambda_{E^{k}}\right)_{q i} m_{H_{1} \tilde{L}_{q}}^{2} \\
& +3\left(\Lambda_{E^{k}}\right)_{q i}\left(\Lambda_{E^{k}}^{\dagger} \Lambda_{D^{p}} Y_{D}^{*}\right)_{j p} m_{H_{1} \tilde{L}_{q}}^{2}-\left(m_{\tilde{L}}^{2 t} \Lambda_{E^{k}}^{\dagger} \Lambda_{E^{p}} \Lambda_{E^{p}}^{\dagger} \Lambda_{E^{k}}\right)_{j i} \\
& -\left(Y_{E^{\prime}}^{\dagger} \Lambda_{E^{p}} \Lambda_{E^{p}}^{\dagger} \Lambda_{E^{k}}\right)_{k i} m_{H_{1} \tilde{L}_{j}}^{2}+\left(Y_{E}^{\dagger}\right)_{k j}\left(\Lambda_{E^{p}} \Lambda_{E^{p}}^{\dagger} \Lambda_{E^{k}}\right)_{q i} m_{H_{1} \tilde{L}_{q}}^{2} \\
& -\left(Y_{E}^{\dagger} \Lambda_{E^{k}}\right)_{p i}\left(\Lambda_{E^{k}}^{\dagger} \Lambda_{E^{p}}\right)_{j l} m_{H_{1} \tilde{L}_{l}}^{2}+\left(\Lambda_{E^{k}}^{\dagger} \Lambda_{E^{p}} Y_{E}^{*}\right)_{j p}\left(\Lambda_{E^{k}}\right)_{q i} m_{H_{1} \tilde{L}_{q}}^{2} \\
& -12\left(\Lambda_{U^{q}} \Lambda_{U^{q}}^{\dagger} m_{\tilde{D}}^{2 t}\right)_{k l}\left(\Lambda_{D^{l}} \Lambda_{D^{k}}^{\dagger}\right)_{i j}-3\left(\Lambda_{D^{k}} D^{t} \Lambda_{D^{k}}^{\dagger} m_{\tilde{L}}^{2}\right)_{i j}-3\left(\left(m_{\tilde{L}}^{2}\right)^{t} \Lambda_{D^{k}}^{*} T \Lambda_{D^{k}}^{t}\right)_{j i} \\
& +3\left(Y_{D}^{\dagger} T \Lambda_{D^{k}}^{t}\right)_{k i} m_{H_{1} \tilde{L}_{j}}^{2}+3\left(\Lambda_{D^{k}} Y_{D}^{*}\right)_{i p}\left(\Lambda_{D^{p}} \Lambda_{D^{k}}^{\dagger}\right)_{q j} m_{H_{1} \tilde{L}_{q}}^{2}-3\left(\Lambda_{D^{k}} \Lambda_{D^{p}}^{\dagger} \Lambda_{D^{p}} \Lambda_{D^{k}}^{\dagger} m_{\tilde{L}}^{2}\right)_{i j} \\
& +3\left(\Lambda_{D^{k}} \Lambda_{D^{p}}^{\dagger} \Lambda_{D^{p}} Y_{D}^{*}\right)_{i k} m_{H_{1} \tilde{L}_{j}}^{2}-6\left(\Lambda_{D^{k}} \Lambda_{D^{p}}^{\dagger} \Lambda_{D^{p}} m_{\tilde{Q}}^{2} \Lambda_{D^{k}}^{\dagger}\right)_{i j} \\
& -6\left(\Lambda_{D^{l}} \Lambda_{D^{k}}^{\dagger} m_{\tilde{L}}^{2}\right)_{i j}\left[\tilde{D}_{l k}+\operatorname{Tr}\left(\Lambda_{D^{l}}^{\dagger} \Lambda_{D^{k}}\right)+\left(\Lambda_{U^{q}} \Lambda_{U^{q}}^{\dagger}\right)_{k l}\right] \\
& +9\left(Y_{D}^{\dagger} D \Lambda_{D^{l}}^{t}\right)_{l i} m_{H_{1} \tilde{L}_{j}}^{2}+6\left(\Lambda_{D^{l}} Y_{D}^{*} \Lambda_{U^{q}} \Lambda_{U^{q}}^{\dagger}\right)_{i l} m_{H_{1} \tilde{L}_{j}}^{2} \\
& +6\left(\Lambda_{D^{l}} Y_{D}^{*}\right)_{i k} \operatorname{Tr}\left(\Lambda_{D^{l}}^{\dagger} \Lambda_{D^{k}}\right) m_{H_{1} \tilde{L}_{j}}^{2}-12\left(m_{\tilde{D}}^{2} \tilde{D}\right)_{l k}\left(\Lambda_{D^{l}} \Lambda_{D^{k}}^{\dagger}\right)_{i j} \\
& +6\left(\Lambda_{D^{l}}^{*} Y_{D}\right)_{q k}\left(\Lambda_{D^{l}} \Lambda_{D^{k}}^{\dagger}\right)_{i j} m_{\tilde{L}_{q} H_{1}}^{2}+6\left(\Lambda_{D^{k}} Y_{D}^{*}\right)_{q l}\left(\Lambda_{D^{l}} \Lambda_{D^{k}}^{\dagger}\right)_{i j} m_{H_{1} \tilde{L}_{q}}^{2}+\frac{3}{5} s^{\prime} \delta_{i j}
\end{aligned}
$$

$$
\begin{aligned}
& \left(16 \pi^{2}\right)^{2} \beta_{\left(M_{\tilde{Q}}^{2}\right)_{i j}}^{(2)}=\left(16 \pi^{2}\right)^{2} \beta_{\left(M_{\tilde{Q}}^{2}\right)_{R P C}}^{(2)} \\
& +\frac{4}{5} g_{1}^{2}\left[2 M_{1}^{2}\left(\Lambda_{D^{q}}^{\dagger} \Lambda_{D^{q}}\right)_{j i}-M_{1}\left(h_{D^{q}}^{\dagger} \Lambda_{D^{q}}\right)_{j i}-M_{1}^{*}\left(\Lambda_{D^{q}}^{\dagger} h_{D^{q}}\right)_{j i}+\left(h_{D^{q}}^{\dagger} h_{D^{q}}\right)_{j i}\right] \\
& -4\left(h_{D^{l}}^{\dagger} h_{D^{m}}\right)_{j i}\left[(\tilde{D})_{m l}+\left(\Lambda_{U^{q}} \Lambda_{U^{q}}^{\dagger}\right)_{l m}+\operatorname{Tr}\left(\Lambda_{D^{m}}^{\dagger} \Lambda_{D^{l}}\right)\right] \\
& -4\left(h_{D^{l}}^{\dagger} \Lambda_{D^{m}}\right)_{j i}\left[\left(Y_{D}^{\dagger} h_{D}\right)_{m l}+\left(h_{U^{q}} \Lambda_{U^{q}}^{\dagger}\right)_{l m}+\operatorname{Tr}\left(\Lambda_{D^{m}}^{\dagger} h_{D^{l}}\right)\right] \\
& -4\left(\Lambda_{D^{l}}^{\dagger} h_{D^{m}}\right)_{j i}\left[\left(h_{D}^{\dagger} Y_{D}\right)_{m l}+\left(\Lambda_{U^{q}} h_{U^{q}}^{\dagger}\right)_{l m}+\operatorname{Tr}\left(h_{D^{m}}^{\dagger} \Lambda_{D^{l}}\right)\right] \\
& -4\left(\Lambda_{D^{l}}^{\dagger} \Lambda_{D^{m}}\right)_{j i}\left[\left(h_{D}^{\dagger} h_{D}\right)_{m l}+\left(h_{U^{q}} h_{U^{q}}^{\dagger}\right)_{l m}+\operatorname{Tr}\left(h_{D^{m}}^{\dagger} h_{D^{l}}\right)\right] \\
& -2\left(h_{D^{m}}^{\dagger} h_{E} Y_{E}^{\dagger} \Lambda_{D^{m}}+h_{D^{m}}^{\dagger} E h_{D^{m}}+\Lambda_{D^{m}}^{\dagger} h_{E} h_{E}^{\dagger} \Lambda_{D^{m}}+\Lambda_{D^{m}}^{\dagger} Y_{E} h_{E}^{\dagger} h_{D^{m}}\right)_{j i} \\
& -6\left(h_{D^{m}}^{\dagger} h_{D^{q}} \Lambda_{D^{q}}^{\dagger} \Lambda_{D^{m}}+h_{D^{m}}^{\dagger} \Lambda_{D^{q}} \Lambda_{D^{q}}^{\dagger} h_{D^{m}}\right)_{j i}-6\left(\Lambda_{D^{m}}^{\dagger} h_{D^{q}} h_{D^{q}}^{\dagger} \Lambda_{D^{m}}+\Lambda_{D^{m}}^{\dagger} \Lambda_{D^{q}} h_{D^{q}}^{\dagger} h_{D^{m}}\right)_{j i} \\
& -2\left(h_{D^{m}}^{\dagger} h_{E^{q}} \Lambda_{E^{q}}^{\dagger} \Lambda_{D^{m}}+h_{D^{m}}^{\dagger} \Lambda_{E^{q}} \Lambda_{E^{q}}^{\dagger} h_{D^{m}}+\Lambda_{D^{m}}^{\dagger} h_{E^{q}} h_{E^{q}}^{\dagger} \Lambda_{D^{m}}+\Lambda_{D^{m}}^{\dagger} \Lambda_{E^{q}} h_{E^{q}}^{\dagger} h_{D^{m}}\right)_{j i}
\end{aligned}
$$




$$
\begin{aligned}
& -2\left(h_{D}\right)_{i l}\left[3 h_{D^{l}}^{\dagger} \Lambda_{D^{m}} Y_{D}^{*}+3 \Lambda_{D^{l}}^{\dagger} \Lambda_{D^{m}} h_{D}^{*}+h_{D^{l}}^{\dagger} \Lambda_{E^{m}} Y_{E}^{*}+\Lambda_{D^{l}}^{\dagger} \Lambda_{E^{m}} h_{E}^{*}\right]_{j m} \\
& -6\left(Y_{D}\right)_{i l}\left[h_{D^{l}}^{\dagger} h_{D^{m}} Y_{D}^{*}+\Lambda_{D^{l}}^{\dagger} h_{D^{m}} h_{D}^{*}\right]_{j m}-2\left(Y_{U}\right)_{i l}\left(Y_{U}^{\dagger}\right)_{k j} \operatorname{Tr}\left(h_{U^{l}}^{\dagger} h_{U^{k}}\right) \\
& -2\left(Y_{D}\right)_{i l}\left(h_{D^{l}}^{\dagger} h_{E^{m}} Y_{E}^{*}+\Lambda_{D^{l}}^{\dagger} h_{E^{m}} h_{E}^{*}\right)_{j m}-4\left(h_{D} h_{U^{q}}^{\dagger} \Lambda_{U^{q}} Y_{D}^{\dagger}\right. \\
& \left.+Y_{D} h_{U q}^{\dagger} h_{U q} Y_{D}^{\dagger}\right)_{i j}-4\left(h_{D} \Lambda_{U q}^{\dagger} \Lambda_{U^{q}} h_{D}^{\dagger}+Y_{D} \Lambda_{U^{q}}^{\dagger} h_{U^{q}} h_{D}^{\dagger}\right)_{i j} \\
& -4\left(h_{D}\right)_{i m}\left(h_{D}^{\dagger}\right)_{l j} \operatorname{Tr}\left(\Lambda_{D^{m}}^{\dagger} \Lambda_{D^{l}}\right)-4\left(Y_{D}\right)_{i m}\left(h_{D}^{\dagger}\right)_{l j} \operatorname{Tr}\left(\Lambda_{D^{m}}^{\dagger} h_{D^{l}}\right) \\
& -4\left(h_{D}\right)_{i m}\left(Y_{D}^{\dagger}\right)_{l j} \operatorname{Tr}\left(h_{D^{m}}^{\dagger} \Lambda_{D^{l}}\right)-4\left(Y_{D}\right)_{i m}\left(Y_{D}^{\dagger}\right)_{l j} \operatorname{Tr}\left(h_{D^{m}}^{\dagger} h_{D^{l}}\right) \\
& -6\left(h_{D}^{*}\right)_{j l}\left(h_{D^{l}}^{t} \Lambda_{D^{m}}^{*} Y_{D}+\Lambda_{D^{l}}^{t} \Lambda_{D^{m}}^{*} h_{D}\right)_{i m}-2\left(h_{D}^{*}\right)_{j l}\left(h_{D^{l}}^{t} \Lambda_{E^{m}}^{*} Y_{E}\right. \\
& \left.+\Lambda_{D^{l}}^{t} \Lambda_{E^{m}}^{*} h_{E}\right)_{i m}-6\left(Y_{D}^{*}\right)_{j l}\left(h_{D^{l}}^{t} h_{D^{m}}^{*} Y_{D}+\Lambda_{D^{l}}^{t} h_{D^{m}}^{*} h_{D}\right)_{i m} \\
& -2\left(Y_{D}^{*}\right)_{j l}\left(h_{D^{l}}^{t} h_{E^{m}}^{*} Y_{E}+\Lambda_{D^{l}}^{t} h_{E^{m}}^{*} h_{E}\right)_{i m}-2\left(h_{U}\right)_{i l}\left(h_{U}^{\dagger}\right)_{k j} \operatorname{Tr}\left(\Lambda_{U^{l}}^{\dagger} \Lambda_{U^{k}}\right) \\
& -2\left(Y_{U}\right)_{i l}\left(h_{U}^{\dagger}\right)_{k j} \operatorname{Tr}\left(\Lambda_{U^{l}}^{\dagger} h_{U^{k}}\right)-2\left(h_{U}\right)_{i l}\left(Y_{U}^{\dagger}\right)_{k j} \operatorname{Tr}\left(h_{U^{l}}^{\dagger} \Lambda_{U^{k}}\right) \\
& +\frac{4}{5} g_{1}^{2}\left[-\left(\Lambda_{D^{k}}\right)_{l i}\left(Y_{D}^{\dagger}\right)_{k j} m_{H_{1} \tilde{L}_{l}}^{2}+\left(\Lambda_{D^{q}}^{\dagger} m_{\tilde{L}}^{2} \Lambda_{D^{q}}\right)_{j i}\right. \\
& \left.+\left(\Lambda_{D^{q}}^{\dagger} \Lambda_{D^{q}} m_{\tilde{Q}}^{2 t}\right)_{j i}+2\left(m_{\tilde{D}}^{2}\right)_{l q}\left(\Lambda_{D^{q}}^{\dagger} \Lambda_{D^{l}}\right)_{j i}-\left(\Lambda_{D^{q}}^{\dagger}\right)_{j m}\left(Y_{D}\right)_{i q} m_{\tilde{L}_{m} H_{1}}^{2}\right] \\
& +4\left(\Lambda_{D^{k}}\right)_{l i}\left(Y_{D}^{\dagger} D\right)_{k j} m_{H_{1} \tilde{L}_{l}}^{2}+4\left(D \Lambda_{D^{q}}^{t}\right)_{i l}\left(Y_{D}^{\dagger}\right)_{q j} m_{H_{1} \tilde{L}_{l}}^{2} \\
& +2 \operatorname{Tr}(E+3 D)\left(\Lambda_{D^{k}}\right)_{l i}\left(Y_{D}^{\dagger}\right)_{k j} m_{H_{1} \tilde{L}_{l}}^{2}+6(D)_{i j}\left(Y_{D}^{\dagger} \Lambda_{D^{k}}^{t}\right)_{k l} m_{H_{1} \tilde{L}_{l}}^{2} \\
& -(D)_{i j}\left(Y_{E}^{\dagger} \Lambda_{E^{k}}\right)_{k l} m_{H_{1} \tilde{L}_{l}}^{2}-2(\tilde{D})_{m l}\left[2\left(\Lambda_{D^{l}}^{\dagger} m_{\tilde{L}}^{2} \Lambda_{D^{m}}\right)_{j i}+\left(\Lambda_{D^{l}}^{\dagger} \Lambda_{D^{m}} m_{\tilde{Q}}^{2}\right)_{j i}\right. \\
& \left.+2\left(m_{\tilde{D}}^{2}\right)_{q m}\left(\Lambda_{D^{l}}^{\dagger} \Lambda_{D^{q}}\right)_{j i}-2\left(\Lambda_{D^{l}}^{\dagger}\right)_{j q}\left(Y_{D}\right)_{i m} m_{\tilde{L}_{q} H_{1}}^{2}\right]-6\left(\Lambda_{D^{m}}^{\dagger} \Lambda_{D^{q}} \Lambda_{D^{q}}^{\dagger} m_{\tilde{L}}^{2} \Lambda_{D^{m}}\right)_{j i} \\
& -2\left(\Lambda_{U^{q}} \Lambda_{U^{q}}^{\dagger}\right)_{l m}\left(2 \Lambda_{D^{l}}^{\dagger} m_{\tilde{L}}^{2} \Lambda_{D^{m}}+\Lambda_{D^{l}}^{\dagger} \Lambda_{D^{m}} m_{\tilde{Q}}^{2}\right)_{j i} \\
& -4\left(m_{\tilde{D}}^{2} \Lambda_{U^{q}}^{\dagger} \Lambda_{U^{q}}\right)_{m l}\left(\Lambda_{D^{l}}^{\dagger} \Lambda_{D^{m}}\right)_{j i}-2 \operatorname{Tr}\left(\Lambda_{D^{m}}^{\dagger} \Lambda_{D^{l}}\right)\left[2\left(\Lambda_{D^{l}}^{\dagger} m_{\tilde{L}}^{2} \Lambda_{D^{m}}\right)_{j i}\right. \\
& \left.+\left(\Lambda_{D^{l}}^{\dagger} \Lambda_{D^{m}} m_{\tilde{Q}}^{2 t}\right)_{j i}+2\left(m_{\tilde{D}}^{2}\right)_{q m}\left(\Lambda_{D^{l}}^{\dagger} \Lambda_{D^{q}}\right)_{j i}-2\left(\Lambda_{D^{l}}^{\dagger}\right)_{j q}\left(Y_{D}\right)_{i m} m_{\tilde{L}_{q} H_{1}}^{2}\right] \\
& +4\left(\Lambda_{D^{l}}^{\dagger} \Lambda_{D^{m}}\right)_{j i}\left(Y_{D^{\dagger}}^{\dagger} \Lambda_{D^{l}}^{t}\right)_{m k} m_{H_{1} \tilde{L}_{k}}^{2}-4\left(\Lambda_{D^{l}}^{\dagger} \Lambda_{D^{m}}\right)_{j i}\left(Y_{D}^{\dagger} m_{\tilde{Q}}^{2} Y_{D}\right. \\
& \left.+Y_{D}^{\dagger} m_{H_{1}}^{2} Y_{D}+\tilde{D} m_{\tilde{D}}^{2}\right)_{m l}-\left(\Lambda_{D^{m}}^{\dagger} \Lambda_{E^{q}} \Lambda_{E^{q}}^{\dagger} \Lambda_{D^{m}} m_{\tilde{Q}}^{2 t}\right)_{j i} \\
& -4\left(\Lambda_{D^{l}}^{\dagger} \Lambda_{D^{m}}\right)_{j i}\left[\left(\Lambda_{U^{q}} m_{\tilde{D}}^{2} \Lambda_{U^{q}}^{\dagger}+m_{\tilde{D}}^{2 t} \Lambda_{U^{q}} \Lambda_{U^{q}}^{\dagger}\right)_{l m}+\left(\Lambda_{U^{k}} \Lambda_{U^{q}}^{\dagger}\right)_{l m}\left(m_{\tilde{U}}^{2}\right)_{k q}\right] \\
& -4\left(\Lambda_{D^{l}}^{\dagger} \Lambda_{D^{m}}\right)_{j i} \operatorname{Tr}\left(\Lambda_{D^{m}}^{\dagger} m_{\tilde{L}}^{2} \Lambda_{D^{l}}+\Lambda_{D^{m}}^{\dagger} \Lambda_{D^{l}} m_{\tilde{Q}}^{2}{ }^{t}\right) \\
& -4\left(\Lambda_{D^{l}}^{\dagger} \Lambda_{D^{m}}\right)_{j i} \operatorname{Tr}\left(\Lambda_{D^{m}}^{\dagger} \Lambda_{D^{k}}\right)\left(m_{\tilde{D}}^{2}\right)_{k l}+4\left(\Lambda_{D^{l}}^{\dagger} \Lambda_{D^{m}}\right)_{j i}\left(Y_{D}^{t} \Lambda_{D^{m}}^{\dagger}\right)_{l k} m_{\tilde{L}_{k} H_{1}}^{2} \\
& -2\left(\Lambda_{D^{m}}^{\dagger} m_{\tilde{L}}^{2} E \Lambda_{D^{m}}+\Lambda_{D^{m}}^{\dagger} Y_{E} m_{\tilde{E}}^{2} Y_{E}^{\dagger} \Lambda_{D^{m}}\right)_{j i}+2\left(\Lambda_{U^{q}} \Lambda_{U^{q}}^{\dagger} Y_{D}^{t}\right)_{l i}\left(\Lambda_{D^{l}}^{\dagger}\right)_{j p} m_{\tilde{L}_{p} H_{1}}^{2} \\
& -2\left(\Lambda_{D^{m}}^{\dagger} E \Lambda_{D^{m}}\right)_{j i} m_{H_{1}}^{2}-2\left(\Lambda_{D^{m}}^{\dagger} \Lambda_{E^{k}}\right)_{j l} m_{H_{1} \tilde{L}_{l}}^{2}\left(Y_{E}^{\dagger} \Lambda_{D^{m}}\right)_{k i} \\
& -2\left(\Lambda_{D^{m}}^{\dagger} E m_{\tilde{L}}^{2} \Lambda_{D^{m}}\right)_{j i}-\left(\Lambda_{D^{m}}^{\dagger} E \Lambda_{D^{m}} m_{\tilde{Q}}^{2 t}\right)_{j i}-2\left(m_{\tilde{D}}^{2}\right)_{k m}\left(\Lambda_{D^{m}}^{\dagger} E \Lambda_{D^{k}}\right)_{j i} \\
& -3\left(\Lambda_{D^{m}}^{\dagger} \Lambda_{D^{q}} \Lambda_{D^{q}}^{\dagger} \Lambda_{D^{m}} m_{\tilde{Q}}^{2}\right)_{j i}-6\left(m_{\tilde{D}}^{2}\right)_{l q}\left(\Lambda_{D^{m}}^{\dagger} \Lambda_{D^{l}} \Lambda_{D^{q}}^{\dagger} \Lambda_{D^{m}}\right)_{j i} \\
& -6\left(m_{\tilde{D}}^{2}\right)_{l m}\left(\Lambda_{D^{m}}^{\dagger} \Lambda_{D^{q}} \Lambda_{D^{q}}^{\dagger} \Lambda_{D^{l}}\right)_{j i}+6\left(\Lambda_{D^{m}}^{\dagger} \Lambda_{D^{q}} \Lambda_{D^{q}}^{\dagger}\right)_{j l}\left(Y_{D}\right)_{i m} m_{\tilde{L}_{l} H_{1}}^{2} \\
& -6\left(\Lambda_{D^{m}}^{\dagger} m_{\tilde{L}}^{2} \Lambda_{D^{q}} \Lambda_{D^{q}}^{\dagger} \Lambda_{D^{m}}+\Lambda_{D^{m}}^{\dagger} \Lambda_{D^{q}} m_{\tilde{Q}}^{2 t} \Lambda_{D^{q}}^{\dagger} \Lambda_{D^{m}}\right)_{j i} \\
& +3\left(\Lambda_{D^{m}}^{\dagger}\right)_{j k}\left(Y_{D}^{t} \Lambda_{D^{q}}^{\dagger} \Lambda_{D^{m}}\right)_{q i} m_{\tilde{L}_{k} H_{1}}^{2}-2\left(\Lambda_{D^{m}}^{\dagger} \Lambda_{E^{q}} \Lambda_{E^{q}}^{\dagger} m_{\tilde{L}}^{2} \Lambda_{D^{m}}\right)_{j i} \\
& -2\left(m_{\tilde{D}}^{2}\right)_{l m}\left(\Lambda_{D^{m}}^{\dagger} \Lambda_{E^{q}} \Lambda_{E^{q}}^{\dagger} \Lambda_{D^{l}}\right)_{j i}+2\left(\Lambda_{D^{m}}^{\dagger} \Lambda_{E^{q}} \Lambda_{E^{q}}^{\dagger}\right)_{j l}\left(Y_{D}\right)_{i m} m_{\tilde{L}_{l} H_{1}}^{2} \\
& -2\left(\Lambda_{D^{m}}^{\dagger} m_{\tilde{L}}^{2} \Lambda_{E^{q}} \Lambda_{E^{q}}^{\dagger} \Lambda_{D^{m}}+\Lambda_{D^{m}}^{\dagger} \Lambda_{E^{q}} m_{\tilde{L}}^{2 t} \Lambda_{E^{q}}^{\dagger} \Lambda_{D^{m}}\right)_{j i}
\end{aligned}
$$




$$
\begin{aligned}
& -2\left(m_{\tilde{E}}^{2}\right)_{l q}\left(\Lambda_{D^{m}}^{\dagger} \Lambda_{E^{l}} \Lambda_{E^{q}}^{\dagger} \Lambda_{D^{m}}\right)_{j i}-2\left(\Lambda_{D^{m}}^{\dagger} Y_{E}\right)_{j q}\left(\Lambda_{E^{q}}^{\dagger} \Lambda_{D^{m}}\right)_{k i} m_{\tilde{L}_{k} H_{1}}^{2} \\
& +\left(\Lambda_{D^{m}}^{\dagger}\right)_{j k}\left(Y_{E}^{t} \Lambda_{E^{q}}^{\dagger} \Lambda_{D^{m}}\right)_{q i} m_{\tilde{L}_{k} H_{1}}^{2}-4\left(Y_{D} \Lambda_{U^{q}}^{\dagger} \Lambda_{U^{l}} Y_{D}^{\dagger}\right)_{i j}\left(m_{\tilde{U}}^{2}\right)_{l q} \\
& -3\left(\Lambda_{D^{l}}^{\dagger} \Lambda_{D^{m}} Y_{D}^{*}\right)_{j m}\left[\left(m_{\tilde{Q}}^{2} Y_{D}+2 Y_{D} m_{\tilde{D}}^{2}\right)_{i l}+2 m_{H_{1}}^{2}\left(Y_{D}\right)_{i l}\right] \\
& +6\left(\Lambda_{D^{l}}^{\dagger} \Lambda_{D^{m}} Y_{D}^{*}\right)_{j m}\left(\Lambda_{D^{l}}\right)_{k i} m_{H_{1} \tilde{L}_{k}}^{2}-2\left(Y_{D}\right)_{i l}\left(\Lambda_{D^{l}}^{\dagger} \Lambda_{E^{k}} Y_{E}^{*} m_{\tilde{E}}^{2 t}\right)_{j k} \\
& -\left(\Lambda_{D^{l}}^{\dagger} \Lambda_{E} m Y_{E}^{*}\right)_{j m}\left[\left(m_{\tilde{Q}}^{2} Y_{D}+2 Y_{D} m_{\tilde{D}}^{2}\right)_{i l}+2 m_{H_{1}}^{2}\left(Y_{D}\right)_{i l}\right. \\
& \left.-2\left(\Lambda_{D^{l}}\right)_{k i} m_{H_{1} \tilde{L}_{k}}^{2}\right]-3\left(Y_{D}\right)_{i l}\left(2 \Lambda_{D^{l}}^{\dagger} m_{\tilde{L}}^{2} \Lambda_{D^{m}} Y_{D}^{*}+\Lambda_{D^{l}}^{\dagger} \Lambda_{D^{m}} m_{\tilde{Q}}^{2}\right)_{j m} \\
& -6\left(Y_{D}\right)_{i l}\left(m_{\tilde{D}}^{2}\right)_{k m}\left(\Lambda_{D^{l}}^{\dagger} \Lambda_{D^{k}} Y_{D}^{*}\right)_{j m}+6\left(Y_{D}\right)_{i l}\left(\Lambda_{D^{l}}^{\dagger}\right)_{j k} \operatorname{Tr}(D) m_{\tilde{L}_{k} H_{1}}^{2} \\
& -2\left(Y_{D}\right)_{i l}\left(\Lambda_{D^{l}}^{\dagger} m_{\tilde{L}}^{2} \Lambda_{E^{m}} Y_{E}^{*}+\Lambda_{D^{l}}^{\dagger} \Lambda_{E^{m}} m_{\tilde{L}}^{2 t} Y_{E}^{*}\right)_{j m} \\
& -2\left(m_{\tilde{Q}}^{2} Y_{D} \Lambda_{U^{q}}^{\dagger} \Lambda_{U^{q}} Y_{D}^{\dagger}+2 Y_{D} m_{\tilde{D}}^{2} \Lambda_{U^{q}}^{\dagger} \Lambda_{U^{q}} Y_{D}^{\dagger}\right)_{i j}-4 m_{H_{1}}^{2}\left(Y_{D} \Lambda_{U^{q}}^{\dagger} \Lambda_{U^{q}} Y_{D}^{\dagger}\right)_{i j} \\
& +2 m_{H_{1} \tilde{L}_{l}}^{2}\left(\Lambda_{D^{k}}\right)_{l i}\left(\Lambda_{U^{q}}^{\dagger} \Lambda_{U^{q}} Y_{D}^{\dagger}\right)_{k j}-4\left(Y_{D} \Lambda_{U^{q}}^{\dagger} \Lambda_{U^{q}} m_{\tilde{D}}^{2} Y_{D}^{\dagger}+Y_{D} \Lambda_{U^{q}}^{\dagger} m_{\tilde{D}}^{2}{ }^{t} \Lambda_{U^{q}} Y_{D}^{\dagger}\right)_{i j} \\
& -2\left(m_{\tilde{Q}}^{2} Y_{D}+2 Y_{D} m_{\tilde{D}}^{2}\right)_{i m}\left(Y_{D}^{\dagger}\right)_{l j} \operatorname{Tr}\left(\Lambda_{D^{m}}^{\dagger} \Lambda_{D^{l}}\right)+2\left(Y_{D}\right)_{i l} \operatorname{Tr}(\tilde{E})\left(\Lambda_{D^{l}}^{\dagger}\right)_{j k} m_{\tilde{L}_{k} H_{1}}^{2} \\
& -4 m_{H_{1}}^{2}\left(Y_{D}\right)_{i m}\left(Y_{D}^{\dagger}\right)_{l j} \operatorname{Tr}\left(\Lambda_{D^{m}}^{\dagger} \Lambda_{D^{l}}\right)+4\left(\Lambda_{D^{m}}\right)_{k i}\left(Y_{D}^{\dagger}\right)_{l j} \operatorname{Tr}\left(\Lambda_{D^{m}}^{\dagger} \Lambda_{D^{l}}\right) m_{H_{1} \tilde{L}_{k}}^{2} \\
& -4\left(Y_{D}\right)_{i m}\left(Y_{D}^{\dagger}\right)_{l j} \operatorname{Tr}\left(\Lambda_{D^{m}}^{\dagger} m_{\tilde{L}}^{2} \Lambda_{D^{l}}+\Lambda_{D^{m}}^{\dagger} \Lambda_{D^{l}} m_{\tilde{Q}}^{2}{ }^{t}\right) \\
& -4\left(m_{\tilde{D}}^{2}\right)_{k l}\left(Y_{D}\right)_{i m}\left(Y_{D}^{\dagger}\right)_{l j} \operatorname{Tr}\left(\Lambda_{D^{m}}^{\dagger} \Lambda_{D^{k}}\right)-6\left(m_{\tilde{D}}^{2} Y_{D}^{\dagger}\right)_{k j}\left(Y_{D}^{t} \Lambda_{D^{m}}^{\dagger} \Lambda_{D^{k}}\right)_{m i} \\
& -6\left(Y_{D}^{\dagger}\right)_{l j}\left(\Lambda_{D^{l}}^{t} \Lambda_{D^{m}}^{*} m_{\tilde{Q}}^{2} Y_{D}+\Lambda_{D^{l}}^{t} \Lambda_{D^{m}}^{*} Y_{D} m_{\tilde{D}}^{2}\right)_{i m} \\
& -6\left(Y_{D}^{*}\right)_{j l} m_{H_{1}}^{2}\left(\Lambda_{D^{l}}^{t} \Lambda_{D^{m}}^{*} Y_{D}\right)_{i m}+6 m_{H_{1} \tilde{L}_{k}}^{2}\left(Y_{D}^{*}\right)_{j l}\left(\Lambda_{D^{l}}^{t} \Lambda_{D^{m}}^{*} \Lambda_{D^{m}}^{t}\right)_{i k} \\
& -3\left(Y_{D}^{\dagger}\right)_{l j}\left(2 Y_{D}^{t} \Lambda_{D^{m}}^{\dagger} m_{\tilde{L}}^{2} \Lambda_{D^{l}}+Y_{D}^{t} \Lambda_{D^{m}}^{\dagger} \Lambda_{D^{l}} m_{\tilde{Q}}^{2 t}\right)_{m i} \\
& +6(D)_{i j} m_{\tilde{L}_{k} H_{1}}^{2}\left(Y_{D}^{t} \Lambda_{D^{m}}^{\dagger}\right)_{m k}-\left(Y_{D}^{\dagger}\right)_{l j}\left(2 Y_{E}^{t} \Lambda_{E^{m}}^{\dagger} m_{\tilde{L}}^{2} \Lambda_{D^{l}}\right. \\
& \left.+Y_{E}^{t} \Lambda_{E m}^{\dagger} \Lambda_{D^{l}} m_{\tilde{Q}}^{2 t}\right)_{m i}+4\left(Y_{D}\right)_{i m}\left(Y_{D}^{\dagger}\right)_{l j}\left(Y_{D}^{t} \Lambda_{D m}^{\dagger}\right)_{l k} m_{\tilde{L}_{k} H_{1}}^{2} \\
& -2\left(m_{\tilde{D}}^{2} Y_{D}^{\dagger}\right)_{k j}\left(Y_{E}^{t} \Lambda_{E^{m}}^{\dagger} \Lambda_{D^{k}}\right)_{m i}+2(D)_{i j} m_{\tilde{L}_{k} H_{1}}^{2}\left(Y_{E}^{t} \Lambda_{E^{m}}^{\dagger}\right)_{m k} \\
& -2\left(Y_{D}^{\dagger}\right)_{l j}\left(\Lambda_{D^{l}}^{t} \Lambda_{E^{m}}^{*} m_{\tilde{L}}^{2} Y_{E}+\Lambda_{D^{l}}^{t} \Lambda_{E^{m}}^{*} Y_{E} m_{\tilde{E}}^{2}\right)_{i m}-\left(Y_{U}^{\dagger} m_{\tilde{Q}}^{2}\right)_{k j}\left(Y_{U}\right)_{i l} \operatorname{Tr}\left(\Lambda_{U^{l}}^{\dagger} \Lambda_{U^{k}}\right) \\
& -2 m_{H_{1}}^{2}\left(Y_{D}^{\dagger}\right)_{l j}\left(\Lambda_{D^{l}}^{t} \Lambda_{E^{m}}^{*} Y_{E}\right)_{i m}-\left(Y_{D}^{\dagger}\right)_{l j}\left(\Lambda_{D^{l}}^{t} \Lambda_{E^{m}}^{*} \Lambda_{E^{m}}\right)_{i k} m_{H_{1} \tilde{L}_{k}}^{2} \\
& -\left[2 m_{H_{2}}^{2}\left(Y_{U}\right)_{i l}+\left(m_{\tilde{Q}}^{2} Y_{U}+2 Y_{U} m_{\tilde{U}}^{2}\right)_{i l}\right]\left(Y_{U}^{\dagger}\right)_{k j} \operatorname{Tr}\left(\Lambda_{U^{l}}^{\dagger} \Lambda_{U^{k}}\right) \\
& -2\left(Y_{U}\right)_{i l}\left(Y_{U}^{\dagger}\right)_{k j}\left[\operatorname{Tr}\left(\Lambda_{U^{l}}^{\dagger} \Lambda_{U^{k}} m_{\tilde{D}}^{2}+m_{\tilde{D}}^{2 t} \Lambda_{U^{k}} \Lambda_{U^{l}}^{\dagger}\right)+\left(m_{\tilde{U}}^{2}\right)_{q k} \operatorname{Tr}\left(\Lambda_{U^{l}}^{\dagger} \Lambda_{U^{q}}\right)\right] \\
& +\frac{2}{5} g_{1}^{2}\left[-\left(Y_{D}\right)_{i k}\left(\Lambda_{D^{k}}^{\dagger}\right)_{j l} m_{\tilde{L}_{l} H_{1}}^{2}+\left(m_{\tilde{Q}}^{2 t} \Lambda_{D^{q}}^{\dagger} \Lambda_{D^{q}}\right)_{j i}\right] \\
& -2\left[(\tilde{D})_{m l}+\left(\Lambda_{U^{q}} \Lambda_{U^{q}}^{\dagger}\right)_{l m}+\operatorname{Tr}\left(\Lambda_{D^{m}}^{\dagger} \Lambda_{D^{l}}\right)\right]\left(m_{\tilde{Q}}^{2}{ }^{t} \Lambda_{D^{l}}^{\dagger} \Lambda_{D^{m}}\right)_{j i} \\
& +2\left(Y_{D}^{*} \Lambda_{U^{q}} \Lambda_{U^{q}}^{\dagger}\right)_{j m}\left(\Lambda_{D^{m}}\right)_{k i} m_{H_{1} \tilde{L}_{k}}^{2}-\left(m_{\tilde{Q}}^{2} \Lambda_{D^{m}}^{\dagger} E \Lambda_{D^{m}}\right)_{j i} \\
& +\left(Y_{D}^{\dagger}\right)_{m j}\left(\Lambda_{E^{q}} \Lambda_{E^{q}}^{\dagger} \Lambda_{D^{m}}\right)_{k i} m_{H_{1} \tilde{L}_{k}}^{2}-\left(m_{\tilde{Q}}^{2 t} \Lambda_{D^{m}}^{\dagger} \Lambda_{E^{q}} \Lambda_{E^{q}}^{\dagger} \Lambda_{D^{m}}\right)_{j i} \\
& -3\left(Y_{D}\right)_{i l}\left(Y_{D}^{\dagger} m_{\tilde{Q}}^{2} \Lambda_{D^{m}}^{t} \Lambda_{D^{l}}^{*}\right)_{m j}-\left(Y_{D}\right)_{i l}\left(m_{\tilde{Q}}^{2}{ }^{t} \Lambda_{D^{l}}^{\dagger} \Lambda_{E} Y_{E}^{*}\right)_{j m} \\
& +(D)_{i j}\left(\Lambda_{E m} Y_{E}^{*}\right)_{k m} m_{H_{1} \tilde{L}_{k}}^{2}-3\left(Y_{D}\right)_{i l}\left(m_{\tilde{Q}}^{2 t} \Lambda_{D^{l}}^{\dagger} \Lambda_{D^{m}} Y_{D}^{*}\right)_{j m} \\
& +2\left(\Lambda_{D^{k}}^{\dagger}\right)_{j l}\left(Y_{D} \Lambda_{U^{q}}^{\dagger} \Lambda_{U^{q}}\right)_{i k} m_{\tilde{L}_{l} H_{1}}^{2}-2\left(Y_{D}\right)_{i m}\left(Y_{D}^{\dagger} m_{\tilde{Q}}^{2}\right)_{l j} \operatorname{Tr}\left(\Lambda_{D^{m}}^{\dagger} \Lambda_{D^{l}}\right) \\
& -\left(Y_{D}^{\dagger} m_{\tilde{Q}}^{2}\right)_{l j}\left[3 \Lambda_{D^{l}}^{t} \Lambda_{D^{m}}^{*} Y_{D}+\Lambda_{D^{l}}^{t} \Lambda_{E^{m}}^{*} Y_{E}\right]_{i m}-2\left(Y_{D} \Lambda_{U^{q}}^{\dagger} \Lambda_{U^{q}} Y_{D}^{\dagger} m_{\tilde{Q}}^{2}\right)_{i j}
\end{aligned}
$$




$$
-3\left(m_{\tilde{Q}}^{2 t} \Lambda_{D^{m}}^{\dagger} \Lambda_{D^{q}} \Lambda_{D^{q}}^{\dagger} \Lambda_{D^{m}}\right)_{j i}+\left(\Lambda_{D^{l}}^{\dagger}\right)_{j k}\left(3 \Lambda_{D^{l}}^{t} \Lambda_{D^{m}}^{*} Y_{D}+\Lambda_{D^{l}}^{t} \Lambda_{E^{m}}^{*} Y_{E}\right)_{i m} m_{\tilde{L}_{k} H_{1}}^{2}-\frac{1}{5} s^{\prime} \delta_{i}
$$

$$
\begin{aligned}
& \left(16 \pi^{2}\right)^{2} \beta_{\left(M_{\tilde{D}}^{2}\right)_{i j}}^{(2)}=\left(16 \pi^{2}\right)^{2} \beta_{\left(M_{\tilde{D}}^{2}\right)_{R P C}}^{(2)}+ \\
& \left(\frac{64}{3} M_{3}^{2} g_{3}^{2}+\frac{64}{15} M_{1}^{2} g_{1}^{2}\right)\left(\Lambda_{U^{q}} \Lambda_{U^{q}}^{\dagger}\right)_{j i}+\left(24 M_{2}^{2} g_{2}^{2}+\frac{8}{5} M_{1}^{2} g_{1}^{2}\right) \operatorname{Tr}\left(\Lambda_{D^{j}} \Lambda_{D^{i}}^{\dagger}\right) \\
& -\left(\frac{32}{3} M_{3} g_{3}^{2}+\frac{32}{15} M_{1} g_{1}^{2}\right)\left(\Lambda_{U^{q}} h_{U^{q}}^{\dagger}\right)_{j i}-\left(12 M_{2} g_{2}^{2}+\frac{4}{5} M_{1} g_{1}^{2}\right) \operatorname{Tr}\left(\Lambda_{D^{j}} h_{D^{i}}^{\dagger}\right) \\
& -\left(\frac{32}{3} M_{3}^{*} g_{3}^{2}+\frac{32}{15} M_{1}^{*} g_{1}^{2}\right)\left(h_{U^{q}} \Lambda_{U^{q}}^{\dagger}\right)_{j i}-\left(12 M_{2}^{*} g_{2}^{2}+\frac{4}{5} M_{1}^{*} g_{1}^{2}\right) \operatorname{Tr}\left(h_{D^{j}} \Lambda_{D^{i}}^{\dagger}\right) \\
& +\left(\frac{32}{3} g_{3}^{2}+\frac{32}{15} g_{1}^{2}\right)\left(h_{U^{q}} h_{U^{q}}^{\dagger}\right)_{j i}+\left(12 g_{2}^{2}+\frac{4}{5} g_{1}^{2}\right) \operatorname{Tr}\left(h_{D^{j}} h_{D^{i}}^{\dagger}\right) \\
& -4\left(h_{D}^{t} h_{D^{p}}^{\dagger} \Lambda_{D^{p}} Y_{D}^{*}+Y_{D}^{t} h_{D^{p}}^{\dagger} h_{D^{p}} Y_{D}^{*}+h_{D}^{t} \Lambda_{D^{p}}^{\dagger} \Lambda_{D^{p}} h_{D}^{*}+Y_{D}^{t} \Lambda_{D^{p}}^{\dagger} h_{D^{p}} h_{D}^{*}\right)_{j i} \\
& -4 \operatorname{Tr}\left(h_{D^{j}}^{t} h_{D^{i}}^{*} D+\Lambda_{D^{j}}^{t} h_{D^{i}}^{*} h_{D} Y_{D}^{\dagger}\right)-4 \operatorname{Tr}\left(h_{D^{j}}^{t} \Lambda_{D^{i}}^{*} Y_{D} h_{D}^{\dagger}+\Lambda_{D^{j}}^{t} \Lambda_{D^{i}}^{*} h_{D} h_{D}^{\dagger}\right) \\
& -4 \operatorname{Tr}\left(h_{D^{j}}^{t} h_{D^{i}}^{*} T+\Lambda_{D^{j}}^{t} h_{D^{i}}^{*} h_{U} Y_{U}^{\dagger}\right)-4 \operatorname{Tr}\left(\Lambda_{D^{i}}^{\dagger} h_{D^{j}} h_{D^{q}}^{\dagger} \Lambda_{D^{q}}+\Lambda_{D^{i}}^{\dagger} \Lambda_{D^{j}} h_{D^{q}}^{\dagger} h_{D^{q}}\right) \\
& -4 \operatorname{Tr}\left(h_{D^{j}}^{t} \Lambda_{D^{i}}^{*} Y_{U} h_{U}^{\dagger}+\Lambda_{D^{j}}^{t} \Lambda_{D^{i}}^{*} h_{U} h_{U}^{\dagger}\right)-4 \operatorname{Tr}\left(h_{D^{i}}^{\dagger} h_{D^{j}} \Lambda_{D^{q}}^{\dagger} \Lambda_{D^{q}}+h_{D^{i}}^{\dagger} \Lambda_{D^{j}} \Lambda_{D^{q}}^{\dagger} h_{D^{q}}\right) \\
& -12 \operatorname{Tr}\left(h_{D^{q}} h_{D^{q}}^{\dagger} \Lambda_{D^{j}} \Lambda_{D^{i}}^{\dagger}+\Lambda_{D^{q}} h_{D^{q}}^{\dagger} h_{D^{j}} \Lambda_{D^{i}}^{\dagger}\right)-12 \operatorname{Tr}\left(h_{D^{q}} \Lambda_{D^{q}}^{\dagger} \Lambda_{D^{j}} h_{D^{i}}^{\dagger}\right. \\
& \left.+\Lambda_{D^{q}} \Lambda_{D^{q}}^{\dagger} h_{D^{j}} h_{D^{i}}^{\dagger}\right)-4 \operatorname{Tr}\left(h_{E^{q}} h_{E^{q}}^{\dagger} \Lambda_{D^{j}} \Lambda_{D^{i}}^{\dagger}+\Lambda_{E^{q}} h_{E^{q}}^{\dagger} h_{D^{j}} \Lambda_{D^{i}}^{\dagger}\right) \\
& -4 \operatorname{Tr}\left(h_{E^{q}} \Lambda_{E^{q}}^{\dagger} \Lambda_{D^{j}} h_{D^{i}}^{\dagger}+\Lambda_{E^{q}} \Lambda_{E^{q}}^{\dagger} h_{D^{j}} h_{D^{i}}^{\dagger}\right)-4 \operatorname{Tr}\left(h_{E} h_{E}^{\dagger} \Lambda_{D^{j}} \Lambda_{D^{i}}^{\dagger}\right. \\
& \left.+Y_{E} h_{E}^{\dagger} h_{D^{j}} \Lambda_{D^{i}}^{\dagger}\right)-4 \operatorname{Tr}\left(h_{E} Y_{E}^{\dagger} \Lambda_{D^{j}} h_{D^{i}}^{\dagger}+E h_{D^{j}} h_{D^{i}}^{\dagger}\right) \\
& -8\left(h_{U^{m}} h_{D}^{\dagger} Y_{D} \Lambda_{U^{m}}^{\dagger}+\Lambda_{U^{m}} h_{D}^{\dagger} h_{D} \Lambda_{U^{m}}^{\dagger}+h_{U^{m}} \tilde{D} h_{U^{m}}^{\dagger}+\Lambda_{U^{m}} Y_{D}^{\dagger} h_{D} h_{U m}^{\dagger}\right)_{j i} \\
& -8\left(h_{U^{m}}^{\dagger} h_{U^{p}} \Lambda_{U^{p}}^{\dagger} \Lambda_{U^{m}}+h_{U^{m}}^{\dagger} \Lambda_{U^{p}} \Lambda_{U^{p}}^{\dagger} h_{U^{m}}+\Lambda_{U^{m}}^{\dagger} h_{U^{p}} h_{U^{p}}^{\dagger} \Lambda_{U^{m}}+\Lambda_{U^{m}}^{\dagger} \Lambda_{U^{p}} h_{U^{p}}^{\dagger} h_{U^{m}}\right)_{i j} \\
& +8\left(h_{U^{m}}^{\dagger}\right)_{i k}\left(h_{U^{m}}\right)_{j l} \operatorname{Tr}\left(\Lambda_{D^{l}} \Lambda_{D^{k}}^{\dagger}\right)+8\left(\Lambda_{U^{m}}^{\dagger}\right)_{i k}\left(\Lambda_{U^{m}}\right)_{j l} \operatorname{Tr}\left(h_{D^{l}} h_{D^{k}}^{\dagger}\right) \\
& +8\left(h_{U^{m}}^{\dagger}\right)_{i k}\left(\Lambda_{U^{m}}\right)_{j l} \operatorname{Tr}\left(h_{D^{l}} \Lambda_{D^{k}}^{\dagger}\right)+8\left(\Lambda_{U^{m}}^{\dagger}\right)_{i k}\left(h_{U^{m}}\right)_{j l} \operatorname{Tr}\left(\Lambda_{D^{l}} h_{D^{k}}^{\dagger}\right) \\
& -12\left(h_{D}^{\dagger} h_{D^{j}}^{t} \Lambda_{D^{p}}^{*} Y_{D}+h_{D^{\prime}}^{\dagger} \Lambda_{D^{j}}^{t} \Lambda_{D^{p}}^{*} h_{D}+Y_{D}^{\dagger} h_{D^{j}}^{t} h_{D^{p}}^{*} Y_{D}+Y_{D^{\dagger}}^{\dagger} \Lambda_{D^{j}}^{t} h_{D^{p}}^{*} h_{D}\right)_{i p} \\
& -4\left(h_{D}^{\dagger} h_{D^{j}}^{t} \Lambda_{E^{p}}^{*} Y_{E}+h_{D^{\dagger}}^{\dagger} \Lambda_{D^{j}}^{t} \Lambda_{E^{p}}^{*} h_{E}+Y_{D^{\dagger}}^{\dagger} h_{D^{j}}^{t} h_{E^{p}}^{*} Y_{E}+Y_{D^{\dagger}}^{\dagger} \Lambda_{D^{j}}^{t} h_{E^{p}}^{*} h_{E}\right)_{i p} \\
& -12\left(h_{D}^{t} h_{D^{i}}^{\dagger} \Lambda_{D^{p}} Y_{D}^{*}+Y_{D}^{t} h_{D^{i}}^{\dagger} h_{D^{p}} Y_{D}^{*}+h_{D}^{t} \Lambda_{D^{i}}^{\dagger} \Lambda_{D^{p}} h_{D}^{*}+Y_{D}^{t} \Lambda_{D^{i}}^{\dagger} h_{D^{p}} h_{D}^{*}\right)_{j p} \\
& -4\left(h_{D}^{t} h_{D^{i}}^{\dagger} \Lambda_{E^{p}} Y_{E}^{*}+Y_{D}^{t} h_{D^{i}}^{\dagger} h_{E^{p}} Y_{E}^{*}+h_{D}^{t} \Lambda_{D^{i}}^{\dagger} \Lambda_{E^{p}} h_{E}^{*}+Y_{D}^{t} \Lambda_{D^{i}}^{\dagger} h_{E^{p}} h_{E}^{*}\right)_{j p} \\
& -4\left(h_{U^{k}}^{\dagger} \Lambda_{U^{l}}\right)_{i j} \operatorname{Tr}\left(\Lambda_{U^{l}}^{\dagger} h_{U^{k}}\right)-4\left(\Lambda_{U^{k}}^{\dagger} h_{U^{l}}\right)_{i j} \operatorname{Tr}\left(h_{U^{l}}^{\dagger} \Lambda_{U^{k}}\right) \\
& -4\left(\Lambda_{U^{k}}^{\dagger} \Lambda_{U^{l}}\right)_{i j} \operatorname{Tr}\left(h_{U^{l}}^{\dagger} h_{U^{k}}\right)-8\left(h_{U^{k}}^{\dagger} h_{U^{l}}\right)_{i j} \tilde{T}_{l k}-4\left(h_{U^{k}}^{\dagger} h_{U^{l}}\right)_{i j} \operatorname{Tr}\left(\Lambda_{U^{l}}^{\dagger} \Lambda_{U^{k}}\right) \\
& -8\left(h_{U^{k}}^{\dagger} \Lambda_{U^{l}}\right)_{i j}\left(Y_{U}^{\dagger} h_{U}\right)_{l k}-8\left(\Lambda_{U^{k}}^{\dagger} h_{U^{l}}\right)_{i j}\left(h_{U}^{\dagger} Y_{U}\right)_{l k}-8\left(\Lambda_{U^{k}}^{\dagger} \Lambda_{U^{l}}\right)_{i j}\left(h_{U}^{\dagger} h_{U}\right)_{l k} \\
& +\left(\frac{16}{3} g_{3}^{2}+\frac{16}{15} g_{1}^{2}\right)\left[2\left(\Lambda_{U^{q}} m_{\tilde{D}}^{2} \Lambda_{U^{q}}^{\dagger}\right)_{j i}+\left(m_{\tilde{D}}^{2 t} \Lambda_{U^{q}} \Lambda_{U^{q}}^{\dagger}\right)_{j i}+2\left(m_{\tilde{U}}^{2}\right)_{l q}\left(\Lambda_{U^{l}} \Lambda_{U^{q}}^{\dagger}\right)_{j i}\right. \\
& \left.+\left(\Lambda_{U^{q}} \Lambda_{U^{q}}^{\dagger} m_{\tilde{D}}^{2 t}\right)_{j i}\right]-\left(6 g_{2}^{2}+\frac{2}{5} g_{1}^{2}\right)\left[\left(\Lambda_{D^{k}}\right)_{l j}\left(Y_{D}^{\dagger}\right)_{k i} m_{H_{1} \tilde{L}_{l}}^{2}+\left(Y_{D}\right)_{j k}\left(\Lambda_{D^{k}}^{\dagger}\right)_{i l} m_{\tilde{L}_{l} H_{1}}^{2}\right] \\
& +\left(6 g_{2}^{2}+\frac{2}{5} g_{1}^{2}\right)\left[2 \operatorname{Tr}\left(m_{\tilde{L}^{2}}^{2} \Lambda_{D^{j}} \Lambda_{D^{i}}^{\dagger}+\Lambda_{D^{j}} m_{\tilde{Q}}^{2} \Lambda_{D^{i}}^{\dagger}\right)+\left(m_{\tilde{D}}^{2}\right)_{l j} \operatorname{Tr}\left(\Lambda_{D^{l}} \Lambda_{D^{i}}^{\dagger}\right)\right. \\
& \left.+\left(m_{\tilde{D}}^{2}\right)_{i l} \operatorname{Tr}\left(\Lambda_{D^{j}} \Lambda_{D^{l}}^{\dagger}\right)\right]-\left(6 g_{2}^{2}+\frac{2}{5} g_{1}^{2}\right)\left[\left(\Lambda_{D^{i}}^{*} Y_{D}\right)_{k j} m_{\tilde{L}_{k} H_{1}}^{2}\right. \\
& \left.+\left(\Lambda_{D^{j}} Y_{D}^{*}\right)_{k i} m_{H_{1} \tilde{L}_{k}}^{2}\right]+4\left(\Lambda_{D^{k}} Y_{D}^{*}\right)_{l i} \tilde{D}_{k j} m_{H_{1} \tilde{L}_{l}}^{2}+4\left(Y_{D}^{\dagger} D \Lambda_{D^{j}}^{t}\right)_{i l} m_{H_{1} \tilde{L}_{l}}^{2} \\
& +4\left(Y_{D}^{\dagger} T \Lambda_{D^{j}}^{t}\right)_{i l} m_{H_{1} \tilde{L}_{l}}^{2}+4\left(Y_{D}^{\dagger} \Lambda_{D^{j}}^{t}\right)_{i l}\left(m_{H_{1} \tilde{L}_{l}}^{2}\right) \operatorname{Tr}(E+3 D)
\end{aligned}
$$




$$
\begin{aligned}
& -4 \tilde{D}_{i j}\left(Y_{E}^{\dagger} \Lambda_{E^{k}}\right)_{k l} m_{H_{1} \tilde{L}_{l}}^{2}+12 \tilde{D}_{i j}\left(Y_{D}^{\dagger} \Lambda_{D^{k}}^{t}\right)_{k l} m_{H_{1} \tilde{L}_{l}}^{2}-4\left(Y_{D}^{\dagger} \Lambda_{D^{p}}^{t} \Lambda_{D^{p}}^{*} m_{\tilde{Q}}^{2} Y_{D}\right)_{i j} \\
& -2\left(Y_{D^{\dagger}}^{\dagger} \Lambda_{D^{p}}^{t} \Lambda_{D^{p}}^{*} Y_{D} m_{\tilde{D}}^{2}\right)_{i j}-4 m_{H_{1}}^{2}\left(Y_{D^{\dagger}}^{\dagger} \Lambda_{D^{p}}^{t} \Lambda_{D^{p}}^{*} Y_{D}\right)_{i j}+4\left(Y_{D^{\dagger}}^{\dagger} \Lambda_{D^{p}}^{t} \Lambda_{D^{p}}^{*} \Lambda_{D^{j}}^{t}\right)_{i l} m_{H_{1} \tilde{L}_{l}}^{2} \\
& -4\left(Y_{D}^{t} \Lambda_{D^{p}}^{\dagger} m_{\tilde{L}}^{2} \Lambda_{D^{p}} Y_{D}^{*}\right)_{j i}-4\left(Y_{D}^{t} \Lambda_{D^{p}}^{\dagger} \Lambda_{D^{p}} m_{\tilde{Q}}^{2 t} Y_{D}^{*}\right)_{j i}-2\left(m_{\tilde{D}}^{2}\right)_{l p}\left(Y_{D}^{t} \Lambda_{D^{p}}^{\dagger} \Lambda_{D^{l}} Y_{D}^{*}\right)_{j i} \\
& +4 \tilde{D}_{i p}\left(\Lambda_{D^{p}}^{*} Y_{D}\right)_{q j} m_{\tilde{L}_{q} H_{1}}^{2}-4 \operatorname{Tr}\left(D^{t} \Lambda_{D^{i}}^{\dagger} m_{\tilde{L}}^{2} \Lambda_{D^{j}}\right)-4 \operatorname{Tr}\left(D^{t} \Lambda_{D^{i}}^{\dagger} \Lambda_{D^{j}} m_{\tilde{Q}}^{2}{ }^{t}\right) \\
& -2\left(m_{\tilde{D}}^{2}\right)_{l j} \operatorname{Tr}\left(D^{t} \Lambda_{D^{i}}^{\dagger} \Lambda_{D^{l}}\right)+4\left(\Lambda_{D^{i}}^{*} D Y_{D}\right)_{k j} m_{\tilde{L}_{k} H_{1}}^{2}-4 \operatorname{Tr}\left(Y_{U}^{*} Y_{U}^{t} \Lambda_{D^{i}}^{\dagger} m_{\tilde{L}}^{2} \Lambda_{D^{j}}\right) \\
& -4 \operatorname{Tr}\left(Y_{U}^{*} Y_{U}^{t} \Lambda_{D^{i}}^{\dagger} \Lambda_{D^{j}} m_{\tilde{Q}}^{2}{ }^{t}\right)-2\left(m_{\tilde{D}}^{2}\right)_{l j} \operatorname{Tr}\left(Y_{U}^{*} Y_{U}^{t} \Lambda_{D^{i}}^{\dagger} \Lambda_{D^{l}}\right)+4\left(\Lambda_{D^{i}}^{*} T Y_{D}\right)_{k j} m_{\tilde{L}_{k} H_{1}}^{2} \\
& -4 \operatorname{Tr}\left(\Lambda_{D^{j}}^{t} \Lambda_{D^{i}}^{*} m_{\tilde{Q}}^{2} D\right)-4 \operatorname{Tr}\left(Y_{D} m_{\tilde{D}}^{2} Y_{D^{\dagger}}^{\dagger} \Lambda_{D^{j}}^{t} \Lambda_{D^{i}}^{*}\right)-4 m_{H_{1}}^{2} \operatorname{Tr}\left(D \Lambda_{D^{j}}^{t} \Lambda_{D^{i}}^{*}\right) \\
& +4\left(Y_{D}^{\dagger} \Lambda_{D^{j}}^{t} \Lambda_{D^{i}}^{*} \Lambda_{D^{k}}^{t}\right){ }_{k l} m_{H_{1} \tilde{L}_{l}}^{2}-4 \operatorname{Tr}\left(m_{\tilde{Q}^{2}}^{2} T \Lambda_{D^{j}}^{t} \Lambda_{D^{i}}^{*}\right)-4 \operatorname{Tr}\left(Y_{U} m_{\tilde{U}^{2}}^{2} Y_{U}^{\dagger} \Lambda_{D^{j}}^{t} \Lambda_{D^{i}}^{*}\right) \\
& -4 m_{H_{2}}^{2} \operatorname{Tr}\left(T \Lambda_{D^{j}}^{t} \Lambda_{D^{i}}^{*}\right)-4 \operatorname{Tr}\left(\Lambda_{D^{q}}^{\dagger} \Lambda_{D^{q}} \Lambda_{D^{i}}^{\dagger} m_{\tilde{L}}^{2} \Lambda_{D^{j}}\right)-4 \operatorname{Tr}\left(\Lambda_{D^{q}}^{\dagger} \Lambda_{D^{q}} \Lambda_{D^{i}}^{\dagger} \Lambda_{D^{j}} m_{\tilde{Q}}^{2}\right) \\
& \left.+4\left(Y_{D}^{t} \Lambda_{D^{q}}^{\dagger} \Lambda_{D^{q}} \Lambda_{D^{i}}^{\dagger}\right)_{j k} m_{\tilde{L}_{k} H_{1}}^{2}-4 \operatorname{Tr}\left(\Lambda_{D^{i}}^{\dagger} \Lambda_{D^{j}} \Lambda_{D^{q}}^{\dagger} \Lambda_{D^{q}} m_{\tilde{Q}}^{2}\right)^{t}\right)-4 \operatorname{Tr}\left(\Lambda_{D^{i}}^{\dagger} \Lambda_{D^{j}} \Lambda_{D^{q}}^{\dagger} m_{\tilde{L}}^{2} \Lambda_{D^{q}}\right) \\
& -4\left(m_{\tilde{D}}^{2}\right)_{l q} \operatorname{Tr}\left(\Lambda_{D^{i}}^{\dagger} \Lambda_{D^{j}} \Lambda_{D^{q}}^{\dagger} \Lambda_{D^{l}}\right)+4\left(Y_{D}^{t} \Lambda_{D^{i}}^{\dagger} \Lambda_{D^{j}} \Lambda_{D^{q}}^{\dagger}\right)_{q k} m_{\tilde{L}_{k} H_{1}}^{2}-12 \operatorname{Tr}\left(\Lambda_{D^{q}}^{\dagger} \Lambda_{D^{j}} \Lambda_{D^{i}}^{\dagger} m_{\tilde{L}^{2}}^{2} \Lambda_{D^{q}}\right) \\
& -12 \operatorname{Tr}\left(\Lambda_{D^{q}}^{\dagger} \Lambda_{D^{j}} \Lambda_{D^{i}}^{\dagger} \Lambda_{D^{q}} m_{\tilde{Q}}^{2 t}\right)-12\left(m_{\tilde{D}}^{2}\right){ }_{l q} \operatorname{Tr}\left(\Lambda_{D^{q}}^{\dagger} \Lambda_{D^{j}} \Lambda_{D^{i}}^{\dagger} \Lambda_{D^{l}}\right) \\
& +12\left(Y_{D}^{t} \Lambda_{D^{q}}^{\dagger} \Lambda_{D^{j}} \Lambda_{D^{i}}^{\dagger}\right)_{q k} m_{\tilde{L}_{k} H_{1}}^{2}-12 \operatorname{Tr}\left(\Lambda_{D^{i}}^{\dagger} \Lambda_{D^{q}} \Lambda_{D^{q}}^{\dagger} m_{\tilde{L}^{2}}^{2} \Lambda_{D^{j}}\right) \\
& -12 \operatorname{Tr}\left(\Lambda_{D^{i}}^{\dagger} \Lambda_{D^{q}} \Lambda_{D^{q}}^{\dagger} \Lambda_{D^{j}} m_{\tilde{Q}}^{2 t}\right)-2\left(m_{\tilde{D}}^{2}\right)_{l j} \operatorname{Tr}\left(\Lambda_{D^{q}}^{\dagger} \Lambda_{D^{q}} \Lambda_{D^{i}}^{\dagger} \Lambda_{D^{l}}\right) \\
& -6\left(m_{\tilde{D}}^{2}\right)_{l j} \operatorname{Tr}\left(\Lambda_{D^{i}}^{\dagger} \Lambda_{D^{q}} \Lambda_{D^{q}}^{\dagger} \Lambda_{D^{l}}\right)+12\left(Y_{D}^{t} \Lambda_{D^{i}}^{\dagger} \Lambda_{D^{q}} \Lambda_{D^{q}}^{\dagger}\right)_{j k} m_{\tilde{L}_{k} H_{1}}^{2} \\
& -4 \operatorname{Tr}\left(m_{\tilde{L}}^{2} \Lambda_{E^{q}} \Lambda_{E^{q}}^{\dagger} \Lambda_{D^{j}} \Lambda_{D^{i}}^{\dagger}\right)-4 \operatorname{Tr}\left(\Lambda_{E^{q}} m_{\tilde{L}}^{2} \Lambda_{E^{q}}^{\dagger} \Lambda_{D^{j}} \Lambda_{D^{i}}^{\dagger}\right) \\
& -4\left(m_{\tilde{E}}^{2}\right)_{l q} \operatorname{Tr}\left(\Lambda_{E^{l}} \Lambda_{E^{q}}^{\dagger} \Lambda_{D^{j}} \Lambda_{D^{i}}^{\dagger}\right)-4\left(\Lambda_{E q}^{\dagger} \Lambda_{D^{j}} \Lambda_{D^{i}}^{\dagger} Y_{E}\right)_{k q} m_{\tilde{L}_{k} H_{1}}^{2} \\
& +4\left(Y_{E}^{t} \Lambda_{E^{q}}^{\dagger} \Lambda_{D^{j}} \Lambda_{D^{i}}^{\dagger}\right)_{q k} m_{\tilde{L}_{k} H_{1}}^{2}-4 \operatorname{Tr}\left(\Lambda_{E^{q}} \Lambda_{E^{q}}^{\dagger} m_{\tilde{L}^{2}}^{2} \Lambda_{D^{j}} \Lambda_{D^{i}}^{\dagger}\right) \\
& -4 \operatorname{Tr}\left(\Lambda_{E^{q}} \Lambda_{E^{q}}^{\dagger} \Lambda_{D^{j}} m_{\tilde{Q}}^{2 t} \Lambda_{D^{i}}^{\dagger}\right)-2\left(m_{\tilde{D}}\right){ }_{l j} \operatorname{Tr}\left(\Lambda_{E^{q}} \Lambda_{E^{q}}^{\dagger} \Lambda_{D^{l}} \Lambda_{D^{i}}^{\dagger}\right) \\
& +4\left(Y_{D}^{t} \Lambda_{D^{i}}^{\dagger} \Lambda_{E^{q}} \Lambda_{E^{q}}^{\dagger}\right)_{j k} m_{\tilde{L}_{k} H_{1}}^{2}-4 \operatorname{Tr}\left(m_{\tilde{L}}^{2} E \Lambda_{D^{j}} \Lambda_{D^{i}}^{\dagger}+Y_{E} m_{\tilde{E}}^{2} Y_{E}^{\dagger} \Lambda_{D^{j}} \Lambda_{D^{i}}^{\dagger}\right) \\
& -4 m_{H_{1}}^{2} \operatorname{Tr}\left(E \Lambda_{D^{j}} \Lambda_{D^{i}}^{\dagger}\right)-4\left(Y_{E}^{\dagger} \Lambda_{D^{j}} \Lambda_{D^{i}}^{\dagger} \Lambda_{E^{k}}\right)_{k l} m_{H_{1} \tilde{L}_{l}}^{2}-4 \operatorname{Tr}\left(\Lambda_{D^{i}}^{\dagger} E m_{\tilde{L}^{2}}^{2} \Lambda_{D^{j}}\right) \\
& -4 \operatorname{Tr}\left(\Lambda_{D^{i}}^{\dagger} E \Lambda_{D^{j}} m_{\tilde{Q}}^{2 t}\right)-2\left(m_{\tilde{D}}^{2}\right)_{l j} \operatorname{Tr}\left(\Lambda_{D^{i}}^{\dagger} E \Lambda_{D^{l}}\right)-4\left(\Lambda_{U^{m}} Y_{D}^{t} \Lambda_{D^{k}}^{\dagger}\right)_{j n}\left(\Lambda_{U^{m}}^{\dagger}\right)_{i k} m_{\tilde{L}_{n} H_{1}}^{2} \\
& -8\left(\Lambda_{U^{m}} m_{\tilde{D}}^{2} \tilde{D} \Lambda_{U^{m}}^{\dagger}\right)_{j i}-4\left(m_{\tilde{D}}^{2}{ }^{t} \Lambda_{U^{m}} \tilde{D} \Lambda_{U^{m}}^{\dagger}\right)_{j i}-8\left(m_{\tilde{U}}^{2}\right)_{l m}\left(\Lambda_{U^{l}} \tilde{D} \Lambda_{U^{m}}^{\dagger}\right)_{j i} \\
& -8\left(\Lambda_{U^{m}} Y_{D}^{\dagger} m_{\tilde{Q}}^{2} Y_{D} \Lambda_{U^{m}}^{\dagger}\right)_{j i}-8\left(\Lambda_{U^{m}} \tilde{D} m_{\tilde{D}}^{2} \Lambda_{U^{m}}^{\dagger}\right)_{j i}-8 m_{H_{1}}^{2}\left(\Lambda_{U^{m}} \tilde{D} \Lambda_{U^{m}}^{\dagger}\right)_{j i} \\
& +4\left(\Lambda_{U^{m}}^{\dagger}\right)_{k i}\left(\Lambda_{U^{m}} Y_{D}^{\dagger} \Lambda_{D^{k}}^{t}\right)_{j l} m_{H_{1} \tilde{L}_{l}}^{2}-8\left(\Lambda_{U^{m}}^{\dagger} \Lambda_{U^{p}} m_{\tilde{D}^{2}}^{2} \Lambda_{U^{p}}^{\dagger} \Lambda_{U^{m}}+\Lambda_{U^{m}}^{\dagger} m_{\tilde{D}}^{2}{ }^{t} \Lambda_{U^{p}} \Lambda_{U^{p}}^{\dagger} \Lambda_{U^{m}}\right)_{i j} \\
& -8\left(\Lambda_{U^{m}}^{\dagger} \Lambda_{U^{l}} \Lambda_{U^{p}}^{\dagger} \Lambda_{U^{m}}\right)_{i j}\left(m_{\tilde{U}}^{2}\right)_{l p}-4\left(\Lambda_{U^{m}}^{\dagger} \Lambda_{U^{p}} \Lambda_{U^{p}}^{\dagger} \Lambda_{U^{m}} m_{\tilde{D}}^{2}\right)_{i j} \\
& -8\left(\Lambda_{U^{m}}^{\dagger} \Lambda_{U^{p}} \Lambda_{U^{p}}^{\dagger} m_{\tilde{D}}^{2}{ }^{t} \Lambda_{U^{m}}\right)_{i j}-8\left(m_{\tilde{U}}^{2}\right)_{l m}\left(\Lambda_{U^{m}}^{\dagger} \Lambda_{U^{p}} \Lambda_{U^{p}}^{\dagger} \Lambda_{U^{l}}\right)_{i j} \\
& +4\left(\Lambda_{U^{m}}^{\dagger}\right)_{i k}\left(\Lambda_{U^{m}} m_{\tilde{D}}^{2}\right)_{j l} \operatorname{Tr}\left(\Lambda_{D^{l}} \Lambda_{D^{k}}^{\dagger}\right)+4\left(\Lambda_{U^{m}}^{\dagger}\right)_{i k}\left(m_{\tilde{D}}^{2}{ }^{t} \Lambda_{U^{m}}\right)_{j l} \operatorname{Tr}\left(\Lambda_{D^{l}} \Lambda_{D^{k}}^{\dagger}\right) \\
& +4\left(\Lambda_{U^{m}}^{\dagger}\right)_{i k}\left(\Lambda_{U^{q}}\right)_{j l}\left(m_{\tilde{U}}^{2}\right)_{q m} \operatorname{Tr}\left(\Lambda_{D^{l}} \Lambda_{D^{k}}^{\dagger}\right)+8\left(\Lambda_{U^{m}}^{\dagger}\right)_{i k}\left(\Lambda_{U^{m}}\right)_{j l} \operatorname{Tr}\left(\Lambda_{D^{l}} m_{\tilde{Q}}^{2}{ }^{t} \Lambda_{D^{k}}^{\dagger}\right. \\
& \left.+m_{\tilde{L}}^{2} \Lambda_{D^{l}} \Lambda_{D^{k}}^{\dagger}\right)+4\left(\Lambda_{U^{m}}^{\dagger}\right)_{i k}\left(\Lambda_{U^{m}} m_{\tilde{D}}^{2}{ }^{t}\right)_{j q} \operatorname{Tr}\left(\Lambda_{D^{q}} \Lambda_{D^{k}}^{\dagger}\right)-12\left(Y_{D}^{\dagger} m_{\tilde{Q}}^{2} \Lambda_{D^{j}}^{t} \Lambda_{D^{p}}^{*} Y_{D}\right)_{i p} \\
& -12\left(Y_{D}^{t} \Lambda_{D^{p}}^{\dagger} m_{\tilde{L}}^{2} \Lambda_{D^{j}} Y_{D}^{*}\right)_{p i}-6\left(m_{\tilde{D}}^{2}\right)_{l j}\left(Y_{D}^{t} \Lambda_{D^{p}}^{\dagger} \Lambda_{D^{l}} Y_{D}^{*}\right)_{p i} \\
& +12 \tilde{D}_{i j}\left(m_{\tilde{L}_{k} H_{1}}^{2}\right)\left(Y_{D}^{t} \Lambda_{D^{p}}^{\dagger}\right)_{p k}-12\left(Y_{D}^{\dagger} \Lambda_{D^{j}}^{t} \Lambda_{D^{p}}^{*} m_{\tilde{Q}}^{2} Y_{D}\right)_{i p} \\
& -6\left(Y_{D}^{\dagger} \Lambda_{D^{j}}^{t} \Lambda_{D^{p}}^{*} Y_{D} m_{\tilde{D}}^{2}\right)_{i p}-12 m_{H_{1}}^{2}\left(Y_{D}^{\dagger} \Lambda_{D^{j}}^{t} \Lambda_{D^{p}}^{*} Y_{D}\right)_{i p}+12\left(Y_{D}^{\dagger} \Lambda_{D^{j}}^{t} \Lambda_{D^{p}}^{*} \Lambda_{D^{p}}^{t}\right)_{i l} m_{H_{1} \tilde{L}_{l}}^{2}
\end{aligned}
$$




$$
\begin{aligned}
& -4\left(Y_{E}^{t} \Lambda_{E^{p}}^{\dagger} m_{\tilde{L}}^{2} \Lambda_{D^{j}} Y_{D}^{*}\right)_{p i}-2\left(m_{\tilde{D}}^{2}\right)_{l j}\left(Y_{E}^{t} \Lambda_{E^{p}}^{\dagger} \Lambda_{D^{l}} Y_{D}^{*}\right)_{p i}+4 \tilde{D}_{i j}\left(Y_{E}^{t} \Lambda_{E^{p}}^{\dagger}\right)_{p k} m_{\tilde{L}_{k} H_{1}}^{2} \\
& -4\left(Y_{D}^{\dagger} \Lambda_{D^{j}}^{t} \Lambda_{E^{p}}^{*} m_{\tilde{L}}^{2} Y_{E}\right)_{i p}-2\left(Y_{D}^{\dagger} \Lambda_{D^{j}}^{t} \Lambda_{E^{p}}^{*} Y_{E} m_{\tilde{E}}^{2}\right)_{i p}-4 m_{H_{1}}^{2}\left(Y_{D^{\dagger}}^{\dagger} \Lambda_{D^{j}}^{t} \Lambda_{E^{p}}^{*} Y_{E}\right)_{i p} \\
& -4\left(Y_{D}^{\dagger} \Lambda_{D^{j}}^{t} \Lambda_{E^{p}}^{*} \Lambda_{E^{p}}\right)_{i l} m_{H_{1} \tilde{L}_{l}}^{2}-12\left(Y_{D}^{\dagger} \Lambda_{D^{p}}^{t} \Lambda_{D^{i}}^{*} m_{\tilde{Q}^{2}}^{2} Y_{D}\right)_{p j}-4\left(Y_{D}^{\dagger} m_{\tilde{Q}^{2}}^{2} \Lambda_{D^{j}}^{t} \Lambda_{E^{p}}^{*} Y_{E}\right)_{i p} \\
& -6\left(Y_{D}^{\dagger} \Lambda_{D^{p}}^{t} \Lambda_{D^{i}}^{*} Y_{D} m_{\tilde{D}}^{2}\right)_{p j}-12 m_{H_{1}}^{2}\left(Y_{D}^{\dagger} \Lambda_{D^{p}}^{t} \Lambda_{D^{i}}^{*} Y_{D}\right)_{p j}+12\left(Y_{D}^{\dagger} \Lambda_{D^{p}}^{t} \Lambda_{D^{i}}^{*} \Lambda_{D^{j}}^{t}\right)_{p l} m_{H_{1} \tilde{L}_{l}}^{2} \\
& -12\left(Y_{D}^{t} \Lambda_{D^{i}}^{\dagger} m_{\tilde{L}}^{2} \Lambda_{D^{p}} Y_{D}^{*}\right)_{j p}-12\left(Y_{D}^{\dagger} m_{\tilde{Q}}^{2} \Lambda_{D^{p}}^{t} \Lambda_{D^{i}}^{*} Y_{D}\right)_{p j} \\
& -6\left(m_{\tilde{D}}^{2}\right)_{l p}\left(Y_{D}^{t} \Lambda_{D^{i}}^{\dagger} \Lambda_{D^{l}} Y_{D}^{*}\right)_{j p}+12 \operatorname{Tr}(D)\left(Y_{D}^{t} \Lambda_{D^{i}}^{\dagger}\right)_{j k} m_{\tilde{L}_{k} H_{1}}^{2} \\
& -4\left(Y_{E}^{\dagger} \Lambda_{E^{p}}^{t} \Lambda_{D^{i}}^{*} m_{\tilde{Q}}^{2} Y_{D}\right)_{p j}-2\left(Y_{E}^{\dagger} \Lambda_{E}^{t} \Lambda_{D^{i}}^{*} Y_{D} m_{\tilde{D}}^{2}\right)_{p j} \\
& -4 m_{H_{1}}^{2}\left(Y_{E}^{\dagger} \Lambda_{E^{p}}^{t} \Lambda_{D^{i}}^{*} Y_{D}\right)_{p j}+4\left(Y_{E}^{\dagger} \Lambda_{E^{p}}^{t} \Lambda_{D^{i}}^{*} \Lambda_{D^{j}}^{t}\right)_{p l} m_{H_{1} \tilde{L}_{l}}^{2} \\
& -4\left(Y_{D}^{t} \Lambda_{D^{i}}^{\dagger} m_{\tilde{L}}^{2} \Lambda_{E^{p}} Y_{E}^{*}\right)_{j p}-4\left(Y_{E}^{\dagger} m_{\tilde{L}}^{2} \Lambda_{E p}^{t} \Lambda_{D^{i}}^{*} Y_{D}\right)_{p j} \\
& -2\left(Y_{D}^{t} \Lambda_{D^{i}}^{\dagger} \Lambda_{E^{l}} Y_{E}^{*}\left(m_{\tilde{E}}^{2}\right)^{t}\right)_{j l}+4\left(Y_{D}^{t} \Lambda_{D^{i}}^{\dagger}\right)_{j k} \operatorname{Tr}(E) m_{\tilde{L}_{k} H_{1}}^{2} \\
& -2\left(\Lambda_{U^{k}}^{\dagger} \Lambda_{U^{l}} m_{\tilde{D}}^{2}\right)_{i j} \operatorname{Tr}\left(\Lambda_{U^{l}}^{\dagger} \Lambda_{U^{k}}\right)-4\left(\Lambda_{U^{k}}^{\dagger} m_{\tilde{D}}^{2}{ }^{t} \Lambda_{U^{l}}\right)_{i j} \operatorname{Tr}\left(\Lambda_{U^{l}}^{\dagger} \Lambda_{U^{k}}\right) \\
& -4\left(m_{\tilde{U}}^{2}\right)_{q l}\left(\Lambda_{U^{k}}^{\dagger} \Lambda_{U^{q}}\right)_{i j} \operatorname{Tr}\left(\Lambda_{U^{l}}^{\dagger} \Lambda_{U^{k}}\right)-8\left(\Lambda_{U^{k}}^{\dagger} \Lambda_{U^{l}}\right)_{i j} \operatorname{Tr}\left(\Lambda_{U^{l}}^{\dagger} \Lambda_{U^{k}} m_{\tilde{D}}^{2}\right) \\
& -4\left(m_{\tilde{U}}^{2}\right)_{q k}\left(\Lambda_{U^{k}}^{\dagger} \Lambda_{U^{l}}\right)_{i j} \operatorname{Tr}\left(\Lambda_{U^{l}}^{\dagger} \Lambda_{U^{q}}\right)-4\left(\Lambda_{U^{k}}^{\dagger} \Lambda_{U^{l}} m_{\tilde{D}}^{2}\right)_{i j} \tilde{T}_{l k} \\
& -8\left(\Lambda_{U^{k}}^{\dagger} m_{\tilde{D}}^{2 t} \Lambda_{U^{l}}\right)_{i j} \tilde{T}_{l k}-8\left(m_{\tilde{U}}^{2} \tilde{T}\right)_{l k}\left(\Lambda_{U^{k}}^{\dagger} \Lambda_{U^{l}}\right)_{i j} \\
& -8\left(\Lambda_{U^{k}}^{\dagger} \Lambda_{U^{l}}\right)_{i j}\left(Y_{U^{\prime}}^{\dagger} m_{\tilde{Q}}^{2} Y_{U}\right)_{l k}-8\left(\Lambda_{U^{k}}^{\dagger} \Lambda_{U^{l}}\right)_{i j}\left(\tilde{T} m_{\tilde{U}}^{2}\right)_{l k} \\
& -8 m_{H_{2}}^{2}\left(\Lambda_{U^{k}}^{\dagger} \Lambda_{U^{l}}\right)_{i j} \tilde{T}_{l k}+4\left(\Lambda_{U^{m}}^{\dagger} m_{\tilde{D}}^{2}\right)_{i q}\left(\Lambda_{U^{m}}\right)_{j l} \operatorname{Tr}\left(\Lambda_{D^{l}} \Lambda_{D^{q}}^{\dagger}\right) \\
& -4\left(\Lambda_{U^{m}}^{\dagger} Y_{D}^{\dagger} \Lambda_{D^{l}}^{t}\right)_{i q}\left(\Lambda_{U^{m}}\right)_{j l} m_{H_{1} \tilde{L}_{q}}^{2}-2\left(m_{\tilde{D}}^{2} Y_{D}^{\dagger} \Lambda_{D^{j}}^{t} \Lambda_{E^{p}}^{*} Y_{E}\right)_{i p} \\
& -2\left(m_{\tilde{D}}^{2}\right)_{i l} \operatorname{Tr}\left(E \Lambda_{D^{j}} \Lambda_{D^{l}}^{\dagger}\right)+4\left(E \Lambda_{D^{j}} Y_{D}^{*}\right)_{k i} m_{H_{1} \tilde{L}_{k}}^{2}-4 \tilde{T}_{l k}\left(m_{\left.\tilde{D}^{2} \Lambda_{U^{k}}^{\dagger} \Lambda_{U^{l}}\right)_{i j}}\right. \\
& -2 \operatorname{Tr}\left(\Lambda_{U^{l}}^{\dagger} \Lambda_{U^{k}}\right)\left(m_{\tilde{D}}^{2} \Lambda_{U^{k}}^{\dagger} \Lambda_{U^{l}}\right)_{i j}-2\left(m_{\tilde{E}}^{2} Y_{E^{\dagger}}^{\dagger} \Lambda_{E^{p}}^{t} \Lambda_{D^{i}}^{*} Y_{D}\right)_{p j}-2\left(m_{\tilde{D}}^{2}\right)_{p l}\left(Y_{D}^{t} \Lambda_{D^{l}}^{\dagger} \Lambda_{D^{p}} Y_{D}^{*}\right)_{j i} \\
& -2\left(m_{\tilde{D}}^{2} Y_{D}^{\dagger} \Lambda_{D^{p}}^{t} \Lambda_{D^{p}}^{*} Y_{D}\right)_{i j}-2\left(m_{\tilde{D}}\right)_{i l} \operatorname{Tr}\left(\Lambda_{D^{j}} D^{t} \Lambda_{D^{l}}^{\dagger}\right) \\
& -2\left(m_{\tilde{D}}^{2}\right)_{i l} \operatorname{Tr}\left(\Lambda_{D^{j}} Y_{U}^{*} Y_{U}^{t} \Lambda_{D^{l}}^{\dagger}\right)-2\left(m_{\tilde{D}}^{2}\right)_{i l} \operatorname{Tr}\left(\Lambda_{D^{l}}^{\dagger} \Lambda_{D^{j}} \Lambda_{D^{q}}^{\dagger} \Lambda_{D^{q}}\right) \\
& -6\left(m_{\tilde{D}}^{2}\right)_{i l} \operatorname{Tr}\left(\Lambda_{D^{q}} \Lambda_{D^{q}}^{\dagger} \Lambda_{D^{j}} \Lambda_{D^{l}}^{\dagger}\right)-2\left(m_{\tilde{D}}^{2}\right)_{i l} \operatorname{Tr}\left(\Lambda_{E^{q}} \Lambda_{E^{q}}^{\dagger} \Lambda_{D^{j}} \Lambda_{D^{l}}^{\dagger}\right) \\
& +4\left(\Lambda_{U^{m}}\right)_{j k}\left(\Lambda_{D^{k}}^{*} Y_{D} \Lambda_{U^{m}}^{\dagger}\right)_{l i} m_{\tilde{L}_{l} H_{1}}^{2}-4\left(\Lambda_{U^{m}} \tilde{D} \Lambda_{U^{m}}^{\dagger} m_{\tilde{D}}^{2}\right)_{j i}^{t}-4\left(m_{\tilde{D}}^{2} \Lambda_{U^{m}}^{\dagger} \Lambda_{U^{p}} \Lambda_{U^{p}}^{\dagger} \Lambda_{U^{m}}\right)_{i j} \\
& +4\left(\Lambda_{U^{m}}\right)_{j l} \operatorname{Tr}\left(\Lambda_{D^{l}} \Lambda_{D^{k}}^{\dagger}\right)\left[\left(m_{\tilde{D}}^{2} \Lambda_{U^{m}}^{\dagger}\right)_{i k}+\left(\Lambda_{U^{m}}^{\dagger} m_{\tilde{D}}^{2 t}\right)_{i k}+\left(m_{\tilde{U}}^{2}\right)_{m q}\left(\Lambda_{U^{q}}^{\dagger}\right)_{i k}\right] \\
& -6\left(m_{\tilde{D}}^{2} Y_{D}^{\dagger} \Lambda_{D^{j}}^{t} \Lambda_{D^{p}}^{*} Y_{D}\right)_{i p}-6\left(Y_{D^{\dagger}}^{\dagger} \Lambda_{D^{j}}^{t} \Lambda_{D^{l}}^{*} Y_{D} m_{\tilde{D}}^{2}\right)_{i l} \\
& -2\left(m_{\tilde{D}}^{2}\right)_{i l}\left(Y_{D}^{t} \Lambda_{D^{l}}^{\dagger} \Lambda_{E^{p}} Y_{E}^{*}\right)_{j p}-6\left(m_{\tilde{D}}^{2} Y_{D}^{\dagger} \Lambda_{D^{p}}^{t} \Lambda_{D^{i}}^{*} Y_{D}\right)_{p j} \\
& -2\left(\left(m_{\tilde{E}}^{2}\right)^{t} Y_{E}^{t} \Lambda_{E^{l}}^{\dagger} \Lambda_{D^{j}} Y_{D}^{*}\right)_{l i}-6\left(m_{\tilde{D}}^{2}\right)_{i l}\left(Y_{D}^{t} \Lambda_{D^{l}}^{\dagger} \Lambda_{D^{p}} Y_{D}^{*}\right)_{j p}-\frac{2}{5} s^{\prime} \delta_{i j}
\end{aligned}
$$

$$
\begin{aligned}
\left(16 \pi^{2}\right)^{2} \beta_{\left(M_{\tilde{U}}^{2}\right)_{i j}}^{(2)}= & \left(16 \pi^{2}\right)^{2} \beta_{\left(M_{\tilde{U}}^{2}\right)_{R P C}}^{(2)}+ \\
& \left(\frac{32}{3} M_{3}^{2} g_{3}^{2}-\frac{16}{15} M_{1}^{2} g_{1}^{2}\right) \operatorname{Tr}\left(\Lambda_{U^{j}} \Lambda_{U^{i}}^{\dagger}\right)-\left(\frac{16}{3} M_{3}^{*} g_{3}^{2}-\frac{8}{15} M_{1}^{*} g_{1}^{2}\right) \operatorname{Tr}\left(h_{U^{j}} \Lambda_{U^{i}}^{\dagger}\right) \\
& +\left(\frac{16}{3} g_{3}^{2}-\frac{8}{15} g_{1}^{2}\right) \operatorname{Tr}\left(h_{U^{j}} h_{U^{i}}^{\dagger}\right)-8\left(h_{U^{i}}^{\dagger} \Lambda_{U^{j}}\right)_{l m} \operatorname{Tr}\left(\Lambda_{D^{m}}^{\dagger} h_{D^{l}}\right) \\
& -4\left(h_{U}^{\dagger} \Lambda_{D^{m}}^{t} \Lambda_{D^{m}}^{*} h_{U}+h_{U}^{\dagger} h_{D^{m}}^{t} \Lambda_{D^{m}}^{*} Y_{U}\right)_{i j}-4\left(Y_{U}^{\dagger} h_{D^{m}}^{t} h_{D^{m}}^{*} Y_{U}+Y_{U}^{\dagger} \Lambda_{D^{m}}^{t} h_{D^{m}}^{*} h_{U}\right)_{i j}
\end{aligned}
$$




$$
\begin{aligned}
& -\left(\frac{16}{3} M_{3} g_{3}^{2}-\frac{8}{15} M_{1} g_{1}^{2}\right) \operatorname{Tr}\left(\Lambda_{U^{j}} h_{U^{i}}^{\dagger}\right)-8\left(h_{U^{i}}^{\dagger} h_{U^{j}}\right)_{l m} \operatorname{Tr}\left(\Lambda_{D^{m}}^{\dagger} \Lambda_{D^{l}}\right) \\
& -8\left(\Lambda_{U^{i}}^{\dagger} h_{U^{j}}\right)_{l m} \operatorname{Tr}\left(h_{D^{m}}^{\dagger} \Lambda_{D^{l}}\right)-8\left(\Lambda_{U^{i}}^{\dagger} \Lambda_{U^{j}}\right)_{l m} \operatorname{Tr}\left(h_{D^{m}}^{\dagger} h_{D^{l}}\right) \\
& -8 \operatorname{Tr}\left(h_{U^{j}} h_{U^{i}}^{\dagger} \Lambda_{U^{p}} \Lambda_{U^{p}}^{\dagger}+\Lambda_{U^{j}} h_{U^{i}}^{\dagger} h_{U^{p}} \Lambda_{U^{p}}^{\dagger}+h_{U^{j}} \Lambda_{U^{i}}^{\dagger} \Lambda_{U^{p}} h_{U^{p}}^{\dagger}+\Lambda_{U^{j}} \Lambda_{U^{i}}^{\dagger} h_{U^{p}} h_{U^{p}}^{\dagger}\right) \\
& -8 \operatorname{Tr}\left(h_{U^{i}}^{\dagger} h_{U^{j}} \tilde{D}+h_{U^{i}}^{\dagger} \Lambda_{U^{j}} Y_{D}^{\dagger} h_{D}\right)-8 \operatorname{Tr}\left(\Lambda_{U^{i}}^{\dagger} h_{U^{j}} h_{D}^{\dagger} Y_{D}+\Lambda_{U^{i}}^{\dagger} \Lambda_{U^{j}} h_{D}^{\dagger} h_{D}\right) \\
& +\left(\frac{8}{3} g_{3}^{2}-\frac{4}{15} g_{1}^{2}\right)\left[4 \operatorname{Tr}\left(\Lambda_{U^{j}} m_{\tilde{D}}^{2} \Lambda_{U^{i}}^{\dagger}\right)+\left(m_{\tilde{U}}^{2}\right)_{i l} \operatorname{Tr}\left(\Lambda_{U^{j}} \Lambda_{U^{l}}^{\dagger}\right)+\left(m_{\tilde{U}}^{2}\right)_{l j} \operatorname{Tr}\left(\Lambda_{U^{l}} \Lambda_{U^{i}}^{\dagger}\right)\right] \\
& +2\left(Y_{U}^{\dagger} \Lambda_{D^{k}}^{t}\right)_{i q}\left(Y_{D}^{\dagger} Y_{U}\right)_{k j} m_{H_{1} \tilde{L}_{q}}^{2}-4\left(Y_{U}^{t} \Lambda_{D^{m}}^{\dagger} m_{\tilde{L}}^{2} \Lambda_{D^{m}} Y_{U}^{*}\right)_{j i} \\
& -4\left(Y_{U}^{t} \Lambda_{D^{m}}^{\dagger} \Lambda_{D^{m}} m_{\tilde{Q}}^{2 t} Y_{U}^{*}\right)_{j i}-4\left(m_{\tilde{D}}^{2}\right)_{l m}\left(Y_{U}^{t} \Lambda_{D^{m}}^{\dagger} \Lambda_{D^{l}} Y_{U}^{*}\right)_{j i} \\
& +4\left(Y_{U}^{\dagger} Y_{D}\right)_{i m}\left(\Lambda_{D^{m}}^{*} Y_{U}\right)_{l j} m_{\tilde{L}_{l} H_{1}}^{2}-4\left(Y_{U}^{\dagger} \Lambda_{D^{m}}^{t} \Lambda_{D^{m}}^{*} m_{\tilde{Q}}^{2} Y_{U}\right)_{i j} \\
& -2\left(Y_{U}^{\dagger} \Lambda_{D^{m}}^{t} \Lambda_{D^{m}}^{*} Y_{U} m_{\tilde{U}}^{2}\right)_{i j}-4 m_{H_{2}}^{2}\left(Y_{U}^{\dagger} \Lambda_{D^{m}}^{t} \Lambda_{D^{m}}^{*} Y_{U}\right)_{i j} \\
& -8 \operatorname{Tr}\left(\Lambda_{D^{m}}^{\dagger} \Lambda_{D^{l}}\right)\left(\Lambda_{U^{i}}^{\dagger} \Lambda_{U^{j}} m_{\tilde{D}}^{2}\right)_{l m}-8 \operatorname{Tr}\left(\Lambda_{D^{m}}^{\dagger} \Lambda_{D^{l}}\right)\left(\Lambda_{U^{i}}^{\dagger} m_{\tilde{D}}^{2 t} \Lambda_{U^{j}}\right)_{l m} \\
& -4\left(m_{\tilde{U}}^{2}\right)_{q j}\left(\Lambda_{U^{i}}^{\dagger} \Lambda_{U^{q}}\right)_{l m} \operatorname{Tr}\left(\Lambda_{D^{m}}^{\dagger} \Lambda_{D^{l}}\right)-8\left(\Lambda_{U^{i}}^{\dagger} \Lambda_{U^{j}}\right)_{l m} \operatorname{Tr}\left(\Lambda_{D^{m}}^{\dagger} m_{\tilde{L}}^{2} \Lambda_{D^{l}}\right) \\
& -8\left(\Lambda_{U^{i}}^{\dagger} \Lambda_{U^{j}}\right)_{l m} \operatorname{Tr}\left(\Lambda_{D^{m}}^{\dagger} \Lambda_{D^{l}} m_{\tilde{Q}}^{2 t}\right)-8\left(m_{\tilde{D}}^{2} \Lambda_{U^{i}}^{\dagger} \Lambda_{U^{j}}\right)_{q m} \operatorname{Tr}\left(\Lambda_{D^{m}}^{\dagger} \Lambda_{D^{q}}\right) \\
& +8\left(\Lambda_{D^{m}}^{*} Y_{D} \Lambda_{U^{i}}^{\dagger} \Lambda_{U^{j}}\right)_{q m} m_{\tilde{L}_{q} H_{1}}^{2}-8 \operatorname{Tr}\left(\Lambda_{U^{j}} m_{\tilde{D}^{2}}^{2} \Lambda_{U^{i}}^{\dagger} \Lambda_{U^{p}} \Lambda_{U^{p}}^{\dagger}\right) \\
& -8 \operatorname{Tr}\left(m_{\tilde{D}}^{2}{ }^{t} \Lambda_{U^{j}} \Lambda_{U^{i}}^{\dagger} \Lambda_{U^{p}} \Lambda_{U^{p}}^{\dagger}\right)-4\left(m_{\tilde{U}}^{2}\right)_{l j} \operatorname{Tr}\left(\Lambda_{U^{l}} \Lambda_{U^{i}}^{\dagger} \Lambda_{U^{p}} \Lambda_{U^{p}}^{\dagger}\right) \\
& -8 \operatorname{Tr}\left(\Lambda_{U^{j}} \Lambda_{U^{i}}^{\dagger} m_{\tilde{D}}^{2}{ }^{t} \Lambda_{U^{p}} \Lambda_{U^{p}}^{\dagger}\right)-8 \operatorname{Tr}\left(\Lambda_{U^{j}} \Lambda_{U^{i}}^{\dagger} \Lambda_{U^{p}} m_{\tilde{D}}^{2} \Lambda_{U^{p}}^{\dagger}\right) \\
& -8\left(m_{\tilde{U}}^{2}\right)_{l p} \operatorname{Tr}\left(\Lambda_{U^{j}} \Lambda_{U^{i}}^{\dagger} \Lambda_{U^{l}} \Lambda_{U^{p}}^{\dagger}\right)-8 \operatorname{Tr}\left(\Lambda_{U^{i}}^{\dagger} \Lambda_{U^{j}} m_{\tilde{D}}^{2} \tilde{D}\right)+8\left(\Lambda_{U^{i}}^{\dagger} \Lambda_{U^{j}} Y_{D}^{\dagger} \Lambda_{D^{k}}^{t}\right)_{k l} m_{H_{1} \tilde{L}_{l}}^{2} \\
& -8 \operatorname{Tr}\left(\Lambda_{U^{i}}^{\dagger} m_{\tilde{D}}^{2}{ }^{t} \Lambda_{U^{j}} \tilde{D}\right)-4 \operatorname{Tr}\left(\Lambda_{U^{i}}^{\dagger} \Lambda_{U^{l}} \tilde{D}\right)\left(m_{\tilde{U}}^{2}\right)_{l j}-8 m_{H_{1}}^{2} \operatorname{Tr}\left(\Lambda_{U^{i}}^{\dagger} \Lambda_{U^{j}} \tilde{D}\right) \\
& -2\left(m_{\tilde{U}}^{2} Y_{U}^{\dagger} \Lambda_{D^{m}}^{t} \Lambda_{D^{m}}^{*} Y_{U}\right)_{i j}+2\left(Y_{D}^{\dagger} Y_{U}\right)_{m j}\left(\Lambda_{D^{m}} Y_{U}^{*}\right)_{q i} m_{H_{1} \tilde{L}_{q}}^{2} \\
& -4\left(m_{\tilde{U}}^{2}\right)_{i q}\left(\Lambda_{U^{q}}^{\dagger} \Lambda_{U^{j}}\right)_{l m} \operatorname{Tr}\left(\Lambda_{D^{m}}^{\dagger} \Lambda_{D^{l}}\right)-4\left(m_{\tilde{U}}^{2}\right)_{i l} \operatorname{Tr}\left(\Lambda_{U^{l}}^{\dagger} \Lambda_{U^{p}} \Lambda_{U^{p}}^{\dagger} \Lambda_{U^{j}}\right) \\
& -8 \operatorname{Tr}\left(\Lambda_{U^{i}}^{\dagger} \Lambda_{U^{j}} Y_{D}^{\dagger} m_{\tilde{Q}}^{2} Y_{D}\right)-8 \operatorname{Tr}\left(\Lambda_{U^{i}}^{\dagger} \Lambda_{U^{j}} \tilde{D} m_{\tilde{D}}^{2}\right)-4\left(m_{\tilde{U}}^{2}\right)_{i l} \operatorname{Tr}\left(\Lambda_{U^{l}}^{\dagger} \Lambda_{U^{j}} \tilde{D}\right)+\frac{4}{5} s^{\prime}\left(\boldsymbol{\Phi}_{i f}\right)
\end{aligned}
$$

$$
\begin{aligned}
& \left(16 \pi^{2}\right)^{2} \beta_{\left(M_{H_{1}}^{2}\right)}^{(2)}=\left(16 \pi^{2}\right)^{2} \beta_{\left(M_{H_{1}}^{2}\right)_{R P C}}^{(2)}+-6 \operatorname{Tr}\left(h_{E} h_{E}^{\dagger} \Lambda_{D^{q}} \Lambda_{D^{q}}^{\dagger}+Y_{E} h_{E}^{\dagger} h_{D^{q}} \Lambda_{D^{q}}^{\dagger}+h_{E} Y_{E}^{\dagger} \Lambda_{D^{q}} h_{D^{q}}^{\dagger}\right. \\
& \left.+E h_{D^{q}} h_{D^{q}}^{\dagger}\right)-2 \operatorname{Tr}\left(h_{E} h_{E}^{\dagger} \Lambda_{E^{q}} \Lambda_{E^{q}}^{\dagger}+Y_{E} h_{E}^{\dagger} h_{E^{q}} \Lambda_{E^{q}}^{\dagger}+h_{E} Y_{E}^{\dagger} \Lambda_{E^{q}} h_{E^{q}}^{\dagger}+E h_{E^{q}} h_{E^{q}}^{\dagger}\right) \\
& -12 \operatorname{Tr}\left(h_{D}^{\dagger} h_{D} \Lambda_{U^{q}}^{\dagger} \Lambda_{U^{q}}+h_{D}^{\dagger} Y_{D} \Lambda_{U^{q}}^{\dagger} h_{U^{q}}+Y_{D}^{\dagger} h_{D} h_{U^{q}}^{\dagger} \Lambda_{U^{q}}+\tilde{D} h_{U^{q}}^{\dagger} h_{U^{q}}\right) \\
& -6 \operatorname{Tr}\left(h_{D} h_{D^{\dagger}}^{\dagger} \Lambda_{D^{q}}^{t} \Lambda_{D^{q}}^{*}+Y_{D} h_{D^{\dagger}}^{\dagger} h_{D^{q}}^{t} \Lambda_{D^{q}}^{*}+h_{D} Y_{D^{\dagger}}^{\dagger} \Lambda_{D^{q}}^{t} h_{D^{q}}^{*}\right. \\
& \left.+D h_{D^{q}}^{t} h_{D^{q}}^{*}\right)-2\left(h_{E}^{\dagger} h_{E}\right)_{i j} \operatorname{Tr}\left(\Lambda_{E^{j}}^{\dagger} \Lambda_{E^{i}}\right)-2\left(h_{E}^{\dagger} Y_{E}\right)_{i j} \operatorname{Tr}\left(\Lambda_{E^{j}}^{\dagger} h_{E^{i}}\right) \\
& -2\left(Y_{E}^{\dagger} h_{E}\right)_{i j} \operatorname{Tr}\left(h_{E^{j}}^{\dagger} \Lambda_{E^{i}}\right)-2(\tilde{E})_{i j} \operatorname{Tr}\left(h_{E^{j}}^{\dagger} h_{E^{i}}\right)-12\left(h_{D}^{\dagger} h_{D}\right)_{i k} \operatorname{Tr}\left(\Lambda_{D^{k}}^{\dagger} \Lambda_{D^{i}}\right) \\
& -12\left(h_{D}^{\dagger} Y_{D}\right)_{i k} \operatorname{Tr}\left(\Lambda_{D^{k}}^{\dagger} h_{D^{i}}\right)-12\left(Y_{D}^{\dagger} h_{D}\right)_{i k} \operatorname{Tr}\left(h_{D^{k}}^{\dagger} \Lambda_{D^{i}}\right) \\
& -12(\tilde{D})_{i k} \operatorname{Tr}\left(h_{D^{k}}^{\dagger} h_{D^{i}}\right)-12 \operatorname{Tr}\left(\Lambda_{D^{k}}^{\dagger} \Lambda_{D^{i}}\right)\left(\tilde{D} m_{\tilde{D}}^{2}+m_{\tilde{D}}^{2} \tilde{D}\right)_{i k} \\
& -\left(16 g_{3}^{2}-\frac{2}{5} g_{1}^{2}\right)\left[\left(\Lambda_{D^{k}}^{*} Y_{D}\right)_{l k} m_{\tilde{L}_{l} H_{1}}^{2}+\left(\Lambda_{D^{k}} Y_{D}^{*}\right)_{l k} m_{H_{1} \tilde{L}_{l}}^{2}\right] \\
& +\frac{6}{5} g_{1}^{2}\left[\left(Y_{E}^{\dagger} \Lambda_{E^{k}}\right)_{k l} m_{H_{1} \tilde{L}_{l}}^{2}+\left(\Lambda_{E^{k}}^{\dagger} Y_{E}\right)_{l k} m_{\tilde{L}_{l} H_{1}}^{2}\right]-6 \operatorname{Tr}\left(Y_{E} m_{\tilde{E}}^{2} Y_{E}^{\dagger} \Lambda_{D^{q}} \Lambda_{D^{q}}^{\dagger}\right) \\
& -6 \operatorname{Tr}\left(E m_{\tilde{L}}^{2} \Lambda_{D^{q}} \Lambda_{D^{q}}^{\dagger}\right)-6 \operatorname{Tr}\left(E \Lambda_{D^{q}}\left(m_{\tilde{Q}}^{2}\right)^{t} \Lambda_{D^{q}}^{\dagger}\right)
\end{aligned}
$$




$$
\begin{aligned}
& -6\left(m_{\tilde{D}}^{2}\right)_{l q} \operatorname{Tr}\left(E \Lambda_{D^{l}} \Lambda_{D^{q}}^{\dagger}\right)-6 \operatorname{Tr}\left(D^{t} \Lambda_{D^{q}}^{\dagger} m_{\tilde{L}}^{2} \Lambda_{D^{q}}\right) \\
& -6\left(m_{\tilde{D}}^{2}\right)_{l q} \operatorname{Tr}\left(D^{t} \Lambda_{D^{q}}^{\dagger} \Lambda_{D^{l}}\right)-12(\tilde{D})_{i k}\left[\operatorname{Tr}\left(\Lambda_{D^{k}}^{\dagger} m_{\tilde{L}}^{2} \Lambda_{D^{i}}\right)\right. \\
& \left.+\operatorname{Tr}\left(\Lambda_{D^{k}}^{\dagger} \Lambda_{D^{i}}\left(m_{\tilde{Q}}^{2}\right)^{t}\right)\right]-9\left(Y_{E} E \Lambda_{E^{k}}\right)_{k l} m_{H_{1} \tilde{L}_{l}}^{2}-2 \operatorname{Tr}\left[m_{\tilde{L}}^{2} E \Lambda_{E^{q}} \Lambda_{E^{q}}^{\dagger}\right] \\
& -2 m_{H_{1}}^{2} \operatorname{Tr}\left(E \Lambda_{E^{q}} \Lambda_{E^{q}}^{\dagger}\right)-12 \operatorname{Tr}\left(Y_{D} \Lambda_{U^{q}}^{\dagger} \Lambda_{U^{q}} Y_{D}^{\dagger} m_{\tilde{Q}}^{2}\right) \\
& -12 m_{H_{1}}^{2} \operatorname{Tr}\left(\tilde{D} \Lambda_{U^{q}}^{\dagger} \Lambda_{U^{q}}\right)-6 m_{H_{1}}^{2} \operatorname{Tr}\left(D \Lambda_{D^{q}}^{t} \Lambda_{D^{q}}^{*}\right) \\
& -6 \operatorname{Tr}\left(Y_{D} m_{\tilde{D}}^{2} Y_{D}^{\dagger} \Lambda_{D^{q}}^{t} \Lambda_{D^{q}}^{*}\right)-12 \operatorname{Tr}\left(\Lambda_{D^{k}}^{\dagger} \Lambda_{D^{i}}\right)\left[\left(Y_{D}^{\dagger} m_{\tilde{Q}}^{2} Y_{D}\right)_{i k}+m_{H_{1}}^{2}(\tilde{D})_{i k}\right] \\
& -2 \operatorname{Tr}\left(\Lambda_{E^{j}}^{\dagger} \Lambda_{E^{i}}\right)\left[\left(Y_{E}^{\dagger} m_{\tilde{L}}^{2} Y_{E}\right)_{i j}+m_{H_{1}}^{2}(\tilde{E})_{i j}\right]-6 \operatorname{Tr}\left(m_{\tilde{Q}^{2}}^{2} \Lambda_{D^{q}}^{t} \Lambda_{D^{q}}^{*} D\right) \\
& -6 \operatorname{Tr}\left(m_{\tilde{Q}}^{2} D \Lambda_{D^{q}}^{t} \Lambda_{D^{q}}^{*}\right)-12 \operatorname{Tr}\left(m_{\tilde{D}}^{2} \Lambda_{U^{q}}^{\dagger} \Lambda_{U^{q}} \tilde{D}\right)-6 \operatorname{Tr}\left(E \Lambda_{D^{q}} \Lambda_{D^{q}}^{\dagger} m_{\tilde{L}}^{2}\right) \\
& -2 \operatorname{Tr}\left(\Lambda_{E q}^{\dagger} E \Lambda_{E^{q}}\left(m_{\tilde{L}}^{2}\right)^{t}\right)-2\left(m_{\tilde{E}}^{2}\right)_{l q} \operatorname{Tr}\left(\Lambda_{E q}^{\dagger} E \Lambda_{E^{l}}\right) \\
& -4(\tilde{E})_{i j} \operatorname{Tr}\left(\Lambda_{E^{j}}^{\dagger} m_{\tilde{L}}^{2} \Lambda_{E^{i}}\right)-12 \operatorname{Tr}\left(\tilde{D} \Lambda_{U^{q}}^{\dagger} m_{\tilde{D}}^{2}{ }^{t} \Lambda_{U^{q}}\right) \\
& -12\left(m_{\tilde{U}}^{2}\right)_{l q} \operatorname{Tr}\left(\tilde{D} \Lambda_{U^{q}}^{\dagger} \Lambda_{U^{l}}\right)-2 \operatorname{Tr}\left(\Lambda_{E^{q}}^{\dagger} E m_{\tilde{L}}^{2} \Lambda_{E q}\right) \\
& -12 \operatorname{Tr}\left(\tilde{D} \Lambda_{U^{q}}^{\dagger} \Lambda_{U^{q}} m_{\tilde{D}}^{2}\right)-2 \operatorname{Tr}\left(\Lambda_{E^{q}} \Lambda_{E^{q}}^{\dagger} Y_{E} m_{\tilde{E}}^{2} Y_{E}^{\dagger}\right)+27 m_{H_{1} \tilde{L}_{l}}^{2}\left(Y_{D}^{\dagger} D \Lambda_{D^{q}}^{t}\right)_{q l} \\
& -\operatorname{Tr}\left(\Lambda_{E^{j}}^{\dagger} \Lambda_{E^{i}}\right)\left(\tilde{E} m_{\tilde{E}}^{2}+m_{\tilde{E}}^{2} \tilde{E}\right)_{i j}+27 m_{\tilde{L}_{l} H_{1}}^{2}\left(\Lambda_{D^{q}}^{*} D Y_{D}\right)_{l q} \\
& +6\left(\Lambda_{U^{q}}^{\dagger} \Lambda_{U^{q}} Y_{D^{\dagger}}^{\dagger} \Lambda_{D^{k}}^{t}\right)_{k l} m_{H_{1} \tilde{L}_{l}}^{2}+3\left(Y_{D^{\dagger}}^{\dagger} \Lambda_{D^{q}}^{t} \Lambda_{D^{q}}^{*} \Lambda_{D^{k}}^{t}\right)_{k l} m_{H_{1} \tilde{L}_{l}}^{2} \\
& -3\left(Y_{E}^{\dagger} \Lambda_{D^{q}} \Lambda_{D^{q}}^{\dagger} \Lambda_{E^{k}}\right)_{k l} m_{H_{1} \tilde{L}_{l}}^{2}-\left(Y_{E}^{\dagger} \Lambda_{E^{q}} \Lambda_{E^{q}}^{\dagger} \Lambda_{E^{k}}\right)_{k l} m_{H_{1} \tilde{L}_{l}}^{2} \\
& +3\left(Y_{D}^{t} \Lambda_{D^{q}}^{\dagger} E\right)_{q l} m_{\tilde{L}_{l} H_{1}}^{2}+\left(Y_{E}^{t} \Lambda_{E^{q}}^{\dagger} E\right)_{q l} m_{\tilde{L}_{l} H_{1}}^{2}-\left(m_{\tilde{E}}^{2} \tilde{E}\right)_{l j} \operatorname{Tr}\left(\Lambda_{E^{j}}^{\dagger} \Lambda_{E^{l}}\right) \\
& -9\left(\Lambda_{E^{k}}^{\dagger} E Y_{E}\right)_{l k} m_{\tilde{L}_{l} H_{1}}^{2}+3\left(\Lambda_{D^{k}}^{*} T Y_{D}\right)_{l k} m_{\tilde{L}_{l} H_{1}}^{2}+3\left(E \Lambda_{D^{q}} Y_{D}^{*}\right)_{i q} m_{H_{1} \tilde{L}_{i}}^{2} \\
& -3\left(\Lambda_{E^{k}}^{\dagger} \Lambda_{D^{q}} \Lambda_{D^{q}}^{\dagger} Y_{E}\right)_{l k} m_{\tilde{L}_{l} H_{1}}^{2}-\left(\Lambda_{E^{k}}^{\dagger} \Lambda_{E^{q}} \Lambda_{E^{q}}^{\dagger} Y_{E}\right)_{l k} m_{\tilde{L}_{l} H_{1}}^{2} \\
& +6\left(\Lambda_{D^{k}}^{*} Y_{D} \Lambda_{U^{q}}^{\dagger} \Lambda_{U^{q}}\right)_{l k} m_{\tilde{L}_{l} H_{1}}^{2}+3\left(\Lambda_{D^{k}}^{*} \Lambda_{D^{q}}^{t} \Lambda_{D^{q}}^{*} Y_{D}\right)_{l k} m_{\tilde{L}_{l} H_{1}}^{2} \\
& +6\left(Y_{D}^{\dagger} \Lambda_{D^{k}}^{t}\right)_{i l} \operatorname{Tr}\left(\Lambda_{D^{k}}^{\dagger} \Lambda_{D^{i}}\right) m_{H_{1} \tilde{L}_{l}}^{2}-\left(Y_{E}^{\dagger} \Lambda_{E^{j}}\right)_{i l} \operatorname{Tr}\left(\Lambda_{E^{j}}^{\dagger} \Lambda_{E^{i}}\right) m_{H_{1} \tilde{L}_{l}}^{2} \\
& -\operatorname{Tr}\left(\Lambda_{E^{j}}^{\dagger} \Lambda_{E^{i}}\right)\left(\Lambda_{E^{i}}^{\dagger} Y_{E}\right)_{l j} m_{\tilde{L}_{l} H_{1}}^{2}+6 \operatorname{Tr}\left(\Lambda_{D^{k}}^{\dagger} \Lambda_{D^{i}}\right)\left(\Lambda_{D^{i}}^{*} Y_{D}\right)_{l k} m_{\tilde{L}_{l} H_{1}}^{2} \\
& +\left(E \Lambda_{E q} Y_{E}^{*}\right)_{l q} m_{H_{1} \tilde{L}_{l}}^{2}-\left(\tilde{E} m_{\tilde{E}}^{2}\right)_{i l} \operatorname{Tr}\left(\Lambda_{E^{i}} \Lambda_{E^{l}}^{\dagger}\right)-6 m_{H_{1}}^{2} \operatorname{Tr}\left(\Lambda_{D^{q}} \Lambda_{D^{q}}^{\dagger} E\right)+\frac{3}{5} s^{\prime}
\end{aligned}
$$

$$
\begin{aligned}
& \left(16 \pi^{2}\right)^{2} \beta_{\left(M_{H_{2}}^{2}\right)}^{(2)}=\left(16 \pi^{2}\right)^{2} \beta_{\left(M_{H_{2}}^{2}\right)_{R P C}}^{(2)}+-6 \operatorname{Tr}\left(h_{U} h_{U}^{\dagger} \Lambda_{D^{q}}^{t} \Lambda_{D^{q}}^{*}+Y_{U} h_{U}^{\dagger} h_{D^{q}}^{t} \Lambda_{D^{q}}^{*}+h_{U} Y_{U^{\dagger}}^{\dagger} \Lambda_{D^{q}}^{t} h_{D^{q}}^{*}+T h_{D^{q}}^{t} h_{D^{q}}^{*}\right) \\
& -6\left(h_{U}^{\dagger} h_{U}\right)_{j k} \operatorname{Tr}\left(\Lambda_{U^{k}}^{\dagger} \Lambda_{U^{j}}\right)-6\left(h_{U}^{\dagger} Y_{U}\right)_{j k} \operatorname{Tr}\left(\Lambda_{U^{k}}^{\dagger} h_{U^{j}}\right)-6\left(Y_{U}^{\dagger} h_{U}\right)_{j k} \operatorname{Tr}\left(h_{U^{k}}^{\dagger} \Lambda_{U^{j}}\right) \\
& -6 \operatorname{Tr}\left(m_{\tilde{Q}}^{2} T \Lambda_{D^{q}}^{t} \Lambda_{D^{q}}^{*}+Y_{U} m_{\tilde{U}}^{2} Y_{U} \Lambda_{D^{q}}^{t} \Lambda_{D^{q}}^{*}\right)-6 m_{H_{2}}^{2} \operatorname{Tr}\left(T \Lambda_{D^{q}}^{t} \Lambda_{D^{q}}^{*}\right) \\
& -6 m_{H_{2}}^{2} \tilde{T}_{j k} \operatorname{Tr}\left(\Lambda_{U^{k}}^{\dagger} \Lambda_{U^{j}}\right)-6\left(Y_{U}^{\dagger} m_{\tilde{Q}}^{2} Y_{U}+\tilde{T} m_{\tilde{U}}^{2}\right)_{j k} \operatorname{Tr}\left(\Lambda_{U^{k}}^{\dagger} \Lambda_{U^{j}}\right) \\
& -6 \operatorname{Tr}\left(m_{\tilde{Q}}^{2} \Lambda_{D^{q}}^{t} \Lambda_{D^{q}}^{*} T\right)-12 \tilde{T}_{j k} \operatorname{Tr}\left(\Lambda_{U^{k}}^{\dagger} \Lambda_{U^{j}} m_{\tilde{D}}^{2}\right)-6 \operatorname{Tr}\left(T \Lambda_{D^{q}}^{t}\left(m_{\tilde{L}}^{2}\right)^{t} \Lambda_{D^{q}}^{*}\right) \\
& -6 \operatorname{Tr}\left(Y_{U}^{*} Y_{U}^{t} \Lambda_{D^{q}}^{\dagger} \Lambda_{D^{l}}\right)\left(m_{\tilde{D}}^{2}\right)_{l q}-6\left(m_{\tilde{U}}^{2} T\right)_{l k} \operatorname{Tr}\left(\Lambda_{U^{k}}^{\dagger} \Lambda_{U^{l}}\right) \\
& +6\left(Y_{D}^{\dagger} T \Lambda_{D^{k}}^{t}\right)_{k l} m_{H_{1} \tilde{L}_{l}}^{2}+6\left(\Lambda_{D^{q}}^{*} T Y_{D}\right)_{i q} m_{\tilde{L}_{i} H_{1}}^{2}-6 \tilde{T}_{j k} \operatorname{Tr}\left(h_{U^{k}}^{\dagger} h_{U^{j}}\right)-\frac{3}{5} s^{\prime} \\
& \left(16 \pi^{2}\right)^{2} \beta_{\left(M_{H_{1} \tilde{L_{i}}}^{2}\right)}^{(2)}=\left(\frac{8}{5} M_{1}^{2} g_{1}^{2}-64 M_{3}^{2} g_{3}^{2}\right)\left(\Lambda_{D^{q}}^{*} Y_{D}\right)_{i q}-\frac{24}{5} M_{1}^{2} g_{1}^{2}\left(\Lambda_{E^{q}}^{*} Y_{E}\right)_{i q}
\end{aligned}
$$




$$
\begin{aligned}
& +\left(-\frac{4}{5} M_{1} g_{1}^{2}+32 M_{3} g_{3}^{2}\right)\left(h_{D^{q}}^{*} Y_{D}\right)_{i q}+\frac{12}{5} M_{1} g_{1}^{2}\left(h_{E^{q}}^{*} Y_{E}\right)_{i q} \\
& +\left(-\frac{4}{5} M_{1}^{*} g_{1}^{2}+32 M_{3}^{*} g_{3}^{2}\right)\left(\Lambda_{D^{q}}^{*} h_{D}\right)_{i q}+\frac{12}{5} M_{1}^{*} g_{1}^{2}\left(\Lambda_{E^{q}}^{*} h_{E}\right)_{i q} \\
& +\left(\frac{4}{5} g_{1}^{2}-32 g_{3}^{2}\right)\left(h_{D^{q}}^{*} h_{D}\right)_{i q}-\frac{12}{5} g_{1}^{2}\left(h_{E^{q}}^{*} h_{E}\right)_{i q}-6\left(h_{E^{j}}^{\dagger} h_{D^{p}} \Lambda_{D^{p}}^{\dagger} Y_{E}\right. \\
& \left.+h_{E j}^{\dagger} \Lambda_{D^{p}} \Lambda_{D^{p}}^{\dagger} h_{E}+\Lambda_{E j}^{\dagger} h_{D^{p}} h_{D^{p}}^{\dagger} Y_{E}+\Lambda_{E j}^{\dagger} \Lambda_{D^{p}} h_{D^{p}}^{\dagger} h_{E}\right)_{i j} \\
& -2\left(h_{E j}^{\dagger} h_{E^{p}} \Lambda_{E^{p}}^{\dagger} Y_{E}+h_{E j}^{\dagger} \Lambda_{E^{p}} \Lambda_{E^{p}}^{\dagger} h_{E}+\Lambda_{E^{j}}^{\dagger} h_{E^{p}} h_{E^{p}}^{\dagger} Y_{E}\right. \\
& \left.+\Lambda_{E^{j}}^{\dagger} \Lambda_{E^{p}} h_{E^{p}}^{\dagger} h_{E}\right)_{i j}-6\left(h_{E j}^{\dagger} h_{E} \tilde{E}+h_{E j}^{\dagger} E h_{E}\right. \\
& \left.+\Lambda_{E^{j}}^{\dagger} h_{E} h_{E}^{\dagger} Y_{E}+\Lambda_{E^{j}}^{\dagger} Y_{E} h_{E}^{\dagger} h_{E}\right)_{i j}-2\left(h_{E^{k}}^{\dagger} h_{E}\right)_{i l} \operatorname{Tr}\left(\Lambda_{E^{l}}^{\dagger} \Lambda_{E^{k}}\right) \\
& -2\left(h_{E^{k}}^{\dagger} Y_{E}\right)_{i l} \operatorname{Tr}\left(\Lambda_{E^{l}}^{\dagger} h_{E^{k}}\right)-2\left(\Lambda_{E^{k}}^{\dagger} h_{E}\right)_{i l} \operatorname{Tr}\left(h_{E^{l}}^{\dagger} \Lambda_{E^{k}}\right) \\
& -6\left(h_{E}^{*} h_{E}^{t} \Lambda_{D^{m}}^{*} Y_{D}+h_{E}^{*} Y_{E}^{t} \Lambda_{D^{m}}^{*} h_{D}+Y_{E}^{*} h_{E}^{t} h_{D^{m}}^{*} Y_{D}\right. \\
& \left.+E^{t} h_{D^{m}}^{*} h_{D}\right)_{i m}+6\left(h_{E}^{*} h_{E}^{t} \Lambda_{E^{m}}^{*} Y_{E}+h_{E}^{*} Y_{E}^{t} \Lambda_{E^{m}}^{*} h_{E}\right. \\
& \left.+Y_{E}^{*} h_{E}^{t} h_{E^{m}}^{*} Y_{E}+E^{t} h_{E^{m}}^{*} h_{E}\right)_{i m}+18\left(h_{D^{k}}^{*} h_{D} \tilde{D}\right. \\
& \left.+h_{D^{k}}^{*} D h_{D}+\Lambda_{D^{k}}^{*} h_{D} h_{D}^{\dagger} Y_{D}+\Lambda_{D^{k}}^{*} Y_{D} h_{D}^{\dagger} h_{D}\right)_{i k} \\
& -12\left(h_{D^{k}}^{*} h_{D} \Lambda_{U^{m}}^{\dagger} \Lambda_{U^{m}}+h_{D^{k}}^{*} Y_{D} \Lambda_{U^{m}}^{\dagger} h_{U^{m}}+\Lambda_{D^{k}}^{*} h_{D} h_{U^{m}}^{\dagger} \Lambda_{U^{m}}\right. \\
& \left.+\Lambda_{D^{k}}^{*} Y_{D} h_{U^{m}}^{\dagger} h_{U^{m}}\right)_{i k}+12\left(h_{D^{k}}^{*} h_{D}\right)_{i l} \operatorname{Tr}\left(\Lambda_{D^{l}} \Lambda_{D^{k}}^{\dagger}\right) \\
& +12\left(\Lambda_{D^{k}}^{*} h_{D}\right)_{i l} \operatorname{Tr}\left(\Lambda_{D^{l}} h_{D^{k}}^{\dagger}\right)+12\left(\Lambda_{D^{k}}^{*} Y_{D}\right)_{i l} \operatorname{Tr}\left(h_{D^{l}} h_{D^{k}}^{\dagger}\right) \\
& +6\left(h_{D^{j}}^{*} h_{U} Y_{U}^{\dagger} Y_{D}+h_{D^{j}}^{*} T h_{D}+\Lambda_{D^{j}}^{*} h_{U} h_{U}^{\dagger} Y_{D}+\Lambda_{D^{j}}^{*} Y_{U} h_{U}^{\dagger} h_{D}\right)_{i j} \\
& +6\left(h_{D^{j}}^{*} h_{D^{m}}^{t} \Lambda_{D^{m}}^{*} Y_{D}+h_{D^{j}}^{*} \Lambda_{D^{m}}^{t} \Lambda_{D^{m}}^{*} h_{D}+\Lambda_{D^{j}}^{*} h_{D^{m}}^{t} h_{D^{m}}^{*} Y_{D}\right. \\
& \left.+\Lambda_{D^{j}}^{*} \Lambda_{D^{m}}^{t} h_{D^{m}}^{*} h_{D}\right)_{i j}-2\left(\Lambda_{E^{k}}^{\dagger} Y_{E}\right)_{i l} \operatorname{Tr}\left(h_{E^{l}}^{\dagger} h_{E^{k}}\right) \\
& +12\left(h_{D^{k}}^{*} Y_{D}\right)_{i l} \operatorname{Tr}\left(h_{D^{l}} \Lambda_{D^{k}}^{\dagger}\right)-3 \operatorname{Tr}\left(Y_{D}^{t} \Lambda_{D^{m}}^{\dagger} \Lambda_{D^{m}} Y_{D}^{*}\right) m_{H_{1} \tilde{L}_{i}}^{2} \\
& +\left(\frac{2}{5} g_{1}^{2}-16 g_{3}^{2}\right)\left[2\left(\Lambda_{D^{q}}^{*} m_{\tilde{Q}}^{2} Y_{D}\right)_{i q}+2\left(\Lambda_{D^{q}}^{*} Y_{D} m_{\tilde{D}}^{2}\right)_{i q}+m_{H_{1}}^{2}\left(\Lambda_{D^{q}}^{*} Y_{D}\right)_{i q}\right. \\
& \left.-\left(\Lambda_{D^{q}}^{*} \Lambda_{D^{q}}^{t}\right)_{i l} m_{H_{1} \tilde{L}_{l}}^{2}\right]-\frac{6}{5} g_{1}^{2}\left[2\left(\Lambda_{E^{q}}^{*} m_{\tilde{L}}^{2} Y_{E}\right)_{i q}+2\left(\Lambda_{E^{q}}^{*} Y_{E} m_{\tilde{E}}^{2}\right)_{i q}\right. \\
& \left.+m_{H_{1}}^{2}\left(\Lambda_{E q}^{*} Y_{E}\right)_{i q}+\left(\Lambda_{E^{q}}^{*} \Lambda_{E^{q}}\right)_{i l} m_{H_{1} \tilde{L}_{l}}^{2}\right]-6\left(\Lambda_{E^{j}}^{\dagger} m_{\tilde{L}}^{2} \Lambda_{D^{p}} \Lambda_{D^{p}}^{\dagger} Y_{E}\right)_{i j} \\
& -6\left(\Lambda_{E^{j}}^{\dagger} \Lambda_{D^{p}} m_{\tilde{Q}}^{2 t} \Lambda_{D p}^{\dagger} Y_{E}\right)_{i j}-6\left(m_{\tilde{D}}^{2}\right)_{l p}\left(\Lambda_{E^{j}}^{\dagger} \Lambda_{D^{l}} \Lambda_{D^{p}}^{\dagger} Y_{E}\right)_{i j} \\
& +3\left(\Lambda_{E j}^{\dagger}\right)_{i q}\left(Y_{D}^{t} \Lambda_{D^{p}}^{\dagger} Y_{E}\right)_{p j} m_{\tilde{L}_{q} H_{1}}^{2}-6\left(\Lambda_{E j}^{\dagger} \Lambda_{D^{p}} \Lambda_{D^{p}}^{\dagger} m_{\tilde{L}}^{2} Y_{E}\right)_{i j} \\
& -3 m_{H_{1}}^{2}\left(\Lambda_{E^{j}}^{\dagger} \Lambda_{D^{p}} \Lambda_{D^{p}}^{\dagger} Y_{E}\right)_{i j}-6\left(\Lambda_{E^{j}}^{\dagger} \Lambda_{D^{p}} \Lambda_{D^{p}}^{\dagger} Y_{E} m_{\tilde{E}}^{2}\right)_{i j} \\
& -3\left(\Lambda_{E^{j}}^{\dagger} \Lambda_{D^{p}} \Lambda_{D^{p}}^{\dagger} \Lambda_{E^{j}}\right)_{i l} m_{H_{1} \tilde{L}_{l}}^{2}-2\left(\Lambda_{E^{j}}^{\dagger} m_{\tilde{L}}^{2} \Lambda_{E^{p}} \Lambda_{E^{p}}^{\dagger} Y_{E}\right)_{i j} \\
& -2\left(\Lambda_{E^{j}}^{\dagger} \Lambda_{E^{p}}\left(m_{\tilde{L}}^{2}\right)^{t} \Lambda_{E^{p}}^{\dagger} Y_{E}\right)_{i j}-2\left(m_{\tilde{E}}^{2}\right)_{l p}\left(\Lambda_{E^{j}}^{\dagger} \Lambda_{E^{l}} \Lambda_{E^{p}}^{\dagger} Y_{E}\right)_{i j} \\
& -2\left(\Lambda_{E^{j}}^{\dagger} \Lambda_{E^{p}} \Lambda_{E^{p}}^{\dagger} m_{\tilde{L}}^{2} Y_{E}\right)_{i j}-2\left(\Lambda_{E^{j}}^{\dagger} \Lambda_{E^{p}} \Lambda_{E^{p}}^{\dagger} Y_{E} m_{\tilde{E}}^{2}\right)_{i j}-6\left(\Lambda_{E^{j}}^{\dagger} m_{\tilde{L}}^{2} E Y_{E}\right)_{i j} \\
& -m_{H_{1}}^{2}\left(\Lambda_{E^{j}}^{\dagger} \Lambda_{E^{p}} \Lambda_{E^{p}}^{\dagger} Y_{E}\right)_{i j}-\left(\Lambda_{E^{j}}^{\dagger} \Lambda_{E^{p}} \Lambda_{E^{p}}^{\dagger} \Lambda_{E^{j}}\right)_{i l} m_{H_{1} \tilde{L}_{l}}^{2}-9 m_{H_{1}}^{2}\left(\Lambda_{E^{j}}^{\dagger} E Y_{E}\right)_{i j} \\
& -6\left(\Lambda_{E j}^{\dagger} Y_{E} m_{\tilde{E}}^{2} \tilde{E}\right)_{i j}-4\left(\Lambda_{E j}^{\dagger} \Lambda_{E^{k}}\right)_{i q} \tilde{E}_{k j} m_{H_{1} \tilde{L}_{q}}^{2}-6\left(\Lambda_{E j}^{\dagger} E m_{\tilde{L}}^{2} Y_{E}\right)_{i j} \\
& -6\left(\Lambda_{E^{j}}^{\dagger} E Y_{E} m_{\tilde{E}}^{2}\right)_{i j}-5\left(\Lambda_{E^{j}}^{\dagger} E \Lambda_{E^{j}}\right)_{i l} m_{H_{1} \tilde{L}_{l}}^{2}-2\left(\Lambda_{E^{k}}^{\dagger} m_{\tilde{L}}^{2} Y_{E}\right)_{i l} \operatorname{Tr}\left(\Lambda_{E^{l}}^{\dagger} \Lambda_{E^{k}}\right) \\
& -2\left(\Lambda_{E^{k}}^{\dagger} Y_{E} m_{\tilde{E}}^{2}\right)_{i l} \operatorname{Tr}\left(\Lambda_{E^{l}}^{\dagger} \Lambda_{E^{k}}\right)-m_{H_{1}}^{2}\left(\Lambda_{E^{k}}^{\dagger} Y_{E}\right)_{i l} \operatorname{Tr}\left(\Lambda_{E^{l}}^{\dagger} \Lambda_{E^{k}}\right) \\
& -\left(\Lambda_{E^{k}}^{\dagger} \Lambda_{E^{l}}\right)_{i q} \operatorname{Tr}\left(\Lambda_{E^{l}}^{\dagger} \Lambda_{E^{k}}\right) m_{H_{1} \tilde{L}_{q}}^{2}-4\left(\Lambda_{E^{k}}^{\dagger} Y_{E}\right)_{i l} \operatorname{Tr}\left(\Lambda_{E^{l}}^{\dagger} m_{\tilde{L}}^{2} \Lambda_{E^{k}}\right)
\end{aligned}
$$




$$
\begin{aligned}
& -2\left(m_{\tilde{E}}^{2}\right)_{q k}\left(\Lambda_{E^{k}}^{\dagger} Y_{E}\right)_{i l} \operatorname{Tr}\left(\Lambda_{E^{l}}^{\dagger} \Lambda_{E^{q}}\right)-6\left(\Lambda_{E^{k}}^{\dagger} Y_{E}\right)_{i l}\left(\Lambda_{E^{l}}^{\dagger} Y_{E}\right)_{q k} m_{\tilde{L}_{q} H_{1}}^{2} \\
& -6\left(Y_{D}^{t} \Lambda_{D^{m}}^{\dagger} m_{\tilde{L}}^{2} E\right)_{m i}-6\left(E^{t} \Lambda_{D^{m}}^{*} Y_{D} m_{\tilde{D}}^{2}\right)_{i m}-6\left(Y_{D}^{t} \Lambda_{D^{m}}^{\dagger} Y_{E} m_{\tilde{E}}^{2} Y_{E}^{\dagger}\right)_{m i} \\
& -9 m_{H_{1}}^{2}\left(Y_{D}^{t} \Lambda_{D^{m}}^{\dagger} E\right)_{m i}-3\left(Y_{D}^{t} \Lambda_{D^{m}}^{\dagger} \Lambda_{E^{k}}\right)_{m q}\left(Y_{E}^{\dagger}\right)_{k i} m_{H_{1} \tilde{L}_{q}}^{2}-6\left(E^{t} \Lambda_{D^{m}}^{*} m_{\tilde{Q}}^{2} Y_{D}\right)_{i m} \\
& +6\left(Y_{E}^{t} \Lambda_{E}^{\dagger} m_{\tilde{L}}^{2} E\right)_{m i}+6\left(Y_{E}^{t} \Lambda_{E}^{\dagger} Y_{E} m_{\tilde{E}}^{2} Y_{E}^{\dagger}\right)_{m i}+4\left(Y_{E}^{t} \Lambda_{E}^{\dagger} Y_{E}\right)_{p j}\left(\Lambda_{E^{j}}^{\dagger}\right)_{i l} m_{\tilde{L}_{l} H_{1}}^{2} \\
& +3\left(Y_{E}^{t} \Lambda_{E^{m}}^{\dagger} \Lambda_{E^{k}}\right)_{m q}\left(Y_{E}^{\dagger}\right)_{k i} m_{H_{1} \tilde{L}_{q}}^{2}+9 m_{H_{1}}^{2}\left(Y_{E}^{t} \Lambda_{E^{m}}^{\dagger} E\right)_{m i}+6\left(E^{t} \Lambda_{E m}^{*} m_{\tilde{L}}^{2} Y_{E}\right)_{i m} \\
& +6\left(E^{t} \Lambda_{E^{m}}^{*} Y_{E} m_{\tilde{E}}^{2}\right)_{i m}+2\left(E^{t} \Lambda_{E^{m}}^{*} \Lambda_{E^{m}}\right)_{i l} m_{H_{1} \tilde{L}_{l}}^{2}+27 m_{H_{1}}^{2}\left(\Lambda_{D^{k}}^{*} D Y_{D}\right)_{i k} \\
& +18\left(\Lambda_{D^{k}}^{*} m_{\tilde{Q}}^{2} D Y_{D}\right)_{i k}+18\left(\Lambda_{D^{k}}^{*} Y_{D} m_{\tilde{D}}^{2} \tilde{D}\right)_{i k}-12\left(\Lambda_{D^{k}}^{*} \Lambda_{D^{m}}^{t}\right)_{i q} \tilde{D}_{m k} m_{H_{1} \tilde{L}_{q}}^{2} \\
& +18\left(\Lambda_{D^{k}}^{*} D m_{\tilde{Q}}^{2} Y_{D}\right)_{i k}+18\left(\Lambda_{D^{k}}^{*} D Y_{D} m_{\tilde{D}}^{2}\right)_{i k}-9\left(\Lambda_{D^{k}}^{*} D \Lambda_{D^{k}}^{t}\right)_{i q} m_{H_{1} \tilde{L}_{q}}^{2} \\
& -12\left(\Lambda_{D^{k}}^{*} m_{\tilde{Q}}^{2} Y_{D} \Lambda_{U^{m}}^{\dagger} \Lambda_{U^{m}}\right)_{i k}-12\left(\Lambda_{D^{k}}^{*} Y_{D} m_{\tilde{D}}^{2} \Lambda_{U^{m}}^{\dagger} \Lambda_{U^{m}}\right)_{i k} \\
& -6 m_{H_{1}}^{2}\left(\Lambda_{D^{k}}^{*} Y_{D} \Lambda_{U^{m}}^{\dagger} \Lambda_{U^{m}}\right)_{i k}+6\left(\Lambda_{D^{k}}^{*} \Lambda_{D^{q}}^{t}\right)_{i j}\left(\Lambda_{U^{m}}^{\dagger} \Lambda_{U^{m}}\right)_{q k} m_{H_{1} \tilde{L}_{j}}^{2} \\
& -12\left(\Lambda_{D^{k}}^{*} Y_{D} \Lambda_{U^{m}}^{\dagger} \Lambda_{U^{m}} m_{\tilde{D}}^{2}\right)_{i k}-12\left(\Lambda_{D^{k}}^{*} Y_{D} \Lambda_{U^{m}}^{\dagger} m_{\tilde{D}}^{2}{ }^{t} \Lambda_{U^{m}}\right)_{i k} \\
& -12\left(m_{\tilde{U}}^{2}\right)_{l m}\left(\Lambda_{D^{k}}^{*} Y_{D} \Lambda_{U^{m}}^{\dagger} \Lambda_{U^{l}}\right)_{i k}+12\left(\Lambda_{D^{k}}^{*} m_{\tilde{Q}}^{2} Y_{D}\right)_{i l} \operatorname{Tr}\left(\Lambda_{D^{l}} \Lambda_{D^{k}}^{\dagger}\right) \\
& +6\left(\Lambda_{D^{k}}^{*} Y_{D} m_{\tilde{D}}^{2}\right)_{i l} \operatorname{Tr}\left(\Lambda_{D^{l}} \Lambda_{D^{k}}^{\dagger}\right)+6 m_{H_{1}}^{2}\left(\Lambda_{D^{k}}^{*} Y_{D}\right)_{i l} \operatorname{Tr}\left(\Lambda_{D^{l}} \Lambda_{D^{k}}^{\dagger}\right) \\
& -6\left(\Lambda_{D^{k}}^{*} \Lambda_{D^{l}}^{t}\right)_{i q} \operatorname{Tr}\left(\Lambda_{D^{l}} \Lambda_{D^{k}}^{\dagger}\right) m_{H_{1} \tilde{L}_{q}}^{2}+12\left(\Lambda_{D^{k}}^{*} Y_{D}\right)_{i l} \operatorname{Tr}\left(m_{\left.\tilde{L}^{2} \Lambda_{D^{l}} \Lambda_{D^{k}}^{\dagger}\right)}\right) \\
& +12\left(\Lambda_{D^{k}}^{*} Y_{D}\right)_{i l} \operatorname{Tr}\left(\Lambda_{D^{l}}\left(m_{\tilde{Q}^{2}}^{2}\right)^{t} \Lambda_{D^{k}}^{\dagger}\right)+6\left(m_{\tilde{D}}^{2} Y_{D}^{t} \Lambda_{D^{k}}^{\dagger}\right)_{q i} \operatorname{Tr}\left(\Lambda_{\left.D^{q} \Lambda_{D^{k}}^{\dagger}\right)}\right) \\
& -6\left(\Lambda_{D^{k}}^{*} Y_{D} Y_{D}^{t} \Lambda_{D^{k}}^{\dagger}\right)_{i q} m_{\tilde{L}_{q} H_{1}}^{2}+6\left(\Lambda_{D^{j}}^{*} m_{\tilde{Q}}^{2} T Y_{D}\right)_{i j}+6\left(\Lambda_{D^{j}}^{*} T Y_{D} m_{\tilde{D}}^{2}\right)_{i j} \\
& +6\left(\Lambda_{D^{j}}^{*} Y_{U} m_{\tilde{U}}^{2} Y_{U}^{\dagger} Y_{D}\right)_{i j}+6 m_{H_{2}}^{2}\left(\Lambda_{D^{j}}^{*} T Y_{D}\right)_{i j}+6\left(\Lambda_{D^{j}}^{*} T m_{\tilde{Q}}^{2} Y_{D}\right)_{i j} \\
& +3 m_{H_{1}}^{2}\left(\Lambda_{D^{j}}^{*} T Y_{D}\right)_{i j}-3\left(\Lambda_{D^{j}}^{*} T \Lambda_{D^{j}}^{t}\right)_{i q} m_{H_{1} \tilde{L}_{q}}^{2} \\
& +6\left(\Lambda_{D^{j}}^{*} \Lambda_{D^{m}}^{t} \Lambda_{D^{m}}^{*} m_{\tilde{Q}}^{2} Y_{D}\right)_{i j}+6\left(\Lambda_{D^{j}}^{*} \Lambda_{D^{m}}^{t} \Lambda_{D^{m}}^{*} Y_{D} m_{\tilde{D}}^{2}\right)_{i j} \\
& +3 m_{H_{1}}^{2}\left(\Lambda_{D^{j}}^{*} \Lambda_{D^{m}}^{t} \Lambda_{D^{m}}^{*} Y_{D}\right)_{i j}-3\left(\Lambda_{D^{j}}^{*} \Lambda_{D^{m}}^{t} \Lambda_{D^{m}}^{*} \Lambda_{D^{j}}^{t}\right)_{i q} m_{H_{1} \tilde{L}_{q}}^{2} \\
& +6\left(Y_{D}^{t} \Lambda_{D^{m}}^{\dagger} m_{\tilde{L}}^{2} \Lambda_{D^{m}} \Lambda_{D^{j}}^{\dagger}\right)_{j i}+6\left(Y_{D}^{t} \Lambda_{D^{m}}^{\dagger} \Lambda_{D^{m}}\left(m_{\tilde{Q}}^{2}\right)^{t} \Lambda_{D^{j}}^{\dagger}\right)_{j i} \\
& +6\left(m_{\tilde{D}}^{2}\right)_{l m}\left(Y_{D}^{t} \Lambda_{D^{m}}^{\dagger} \Lambda_{D^{l}} \Lambda_{D^{j}}^{\dagger}\right)_{j i}+\left(\frac{2}{5} g_{1}^{2}-16 g_{3}^{2}\right)\left[\left(Y_{D}^{t} \Lambda_{D^{q}}^{\dagger} m_{\tilde{L}}^{2}\right)_{q i}\right. \\
& \left.-m_{H_{1} \tilde{L}_{i}}^{2} \operatorname{Tr}(D)\right]-\frac{6}{5} g_{1}^{2}\left[\left(Y_{E}^{t} \Lambda_{E^{q}}^{\dagger} m_{\tilde{L}}^{2}\right)_{q i}+(E)_{k i} m_{H_{1} \tilde{L}_{k}}^{2}-m_{H_{1} \tilde{L}_{i}}^{2} \operatorname{Tr}(E)\right] \\
& -3\left(m_{\tilde{L}}^{2 t} \Lambda_{E^{j}}^{\dagger} \Lambda_{D^{p}} \Lambda_{D^{p}}^{\dagger} Y_{E}\right)_{i j}-3 \operatorname{Tr}\left(Y_{E}^{\dagger} \Lambda_{D^{p}} \Lambda_{D^{p}}^{\dagger} Y_{E}\right) m_{H_{1} \tilde{L}_{i}}^{2} \\
& +6\left(\Lambda_{D^{p}} \Lambda_{D^{p}}^{\dagger} E\right)_{q i} m_{H_{1} \tilde{L}_{q}}^{2}+3\left(\Lambda_{E^{j}}^{\dagger} \Lambda_{D^{p}} Y_{D}^{*}\right)_{i p}\left(Y_{E}\right)_{q j} m_{H_{1} \tilde{L}_{q}}^{2} \\
& -\left(\left(m_{\tilde{L}}^{2}\right)^{t} \Lambda_{E^{j}}^{\dagger} \Lambda_{E^{p}} \Lambda_{E^{p}}^{\dagger} Y_{E}\right)_{i j}-\operatorname{Tr}\left(Y_{E}^{\dagger} \Lambda_{E^{p}} \Lambda_{E p}^{\dagger} Y_{E}\right) m_{H_{1} \tilde{L}_{i}}^{2} \\
& +\left(\Lambda_{E^{j}}^{\dagger} \Lambda_{E^{p}} Y_{E}^{*}\right)_{i p}\left(Y_{E}\right)_{q j} m_{H_{1} \tilde{L}_{q}}^{2}-3\left(m_{\tilde{L}}^{2}{ }^{t} \Lambda_{E^{j}}^{\dagger} E Y_{E}\right)_{i j}-3 \operatorname{Tr}\left(E^{2}\right) m_{H_{1} \tilde{L}_{i}}^{2} \\
& +6\left(E^{2}\right)_{q i} m_{H_{1} \tilde{L}_{q}}^{2}-\left(\left(m_{\tilde{L}}^{2}\right)^{t} \Lambda_{E^{k}}^{\dagger} Y_{E}\right)_{i l} \operatorname{Tr}\left(\Lambda_{E^{l}}^{\dagger} \Lambda_{E^{k}}\right)+3\left(Y_{E}^{t} \Lambda_{E^{m}}^{\dagger} E m_{\tilde{L}}^{2}\right)_{m i} \\
& -\tilde{E}_{l k} \operatorname{Tr}\left(\Lambda_{E^{l}}^{\dagger} \Lambda_{E^{k}}\right) m_{H_{1} \tilde{L}_{i}}^{2}+\left(Y_{E}^{\dagger}\right)_{k i}\left(Y_{E}\right)_{q l} \operatorname{Tr}\left(\Lambda_{E^{l}}^{\dagger} \Lambda_{E^{k}}\right) m_{H_{1} \tilde{L}_{q}}^{2} \\
& -3\left(Y_{D}^{t} \Lambda_{D^{m}}^{\dagger} E m_{\tilde{L}}^{2}\right)_{m i}-3\left(Y_{D}^{t} \Lambda_{D^{m}}^{\dagger} Y_{E}\right)_{m k}\left(\Lambda_{E^{k}}^{\dagger}\right)_{l i} m_{\tilde{L}_{l} H_{1}}^{2} \\
& -3 \operatorname{Tr}(E)(E)_{q i} m_{H_{1} \tilde{L}_{q}}^{2}+9\left(Y_{D}^{t} D^{t} \Lambda_{D^{k}}^{\dagger} m_{\tilde{L}}^{2}\right)_{k i}-9 \operatorname{Tr}\left(D^{2}\right) m_{H_{1} \tilde{L}_{i}}^{2} \\
& -12\left(\Lambda_{D^{k}}^{*} Y_{D}\right)_{i l}\left(\Lambda_{D^{l}}^{*} Y_{D}\right)_{q k} m_{\tilde{L}_{q} H_{1}}^{2}-6\left(\Lambda_{U^{m}}^{t} \Lambda_{U^{m}}^{*} Y_{D}^{t} \Lambda_{D^{k}}^{\dagger} m_{\tilde{L}}^{2}\right)_{k i}
\end{aligned}
$$




$$
\begin{aligned}
& +6 \operatorname{Tr}\left(\tilde{D} \Lambda_{U^{m}}^{\dagger} \Lambda_{U^{m}}\right) m_{H_{1} \tilde{L}_{i}}^{2}+6\left(Y_{D}^{t} \Lambda_{D^{k}}^{\dagger} m_{\tilde{L}}^{2}\right)_{l i} \operatorname{Tr}\left(\Lambda_{D^{l}} \Lambda_{D^{k}}^{\dagger}\right) \\
& +12\left(m_{\tilde{D}}^{2}\right)_{k q}\left(\Lambda_{D^{q}}^{*} Y_{D}\right)_{i l} \operatorname{Tr}\left(\Lambda_{D^{l}} \Lambda_{D^{k}}^{\dagger}\right)-6 \tilde{D}_{k l} \operatorname{Tr}\left(\Lambda_{D^{l}} \Lambda_{D^{k}}^{\dagger}\right) m_{H_{1} \tilde{L}_{i}}^{2} \\
& -6 m_{H_{1} \tilde{L}_{j}}^{2}\left(\Lambda_{D^{l}} Y_{D}^{*}\right)_{j k}\left(\Lambda_{D^{k}}^{*} Y_{D}\right)_{i l}+3\left(Y_{D^{\prime}}^{t} T^{t} \Lambda_{D^{j}}^{\dagger} m_{\tilde{L}}^{2}\right)_{j i} \\
& -3 \operatorname{Tr}(D T) m_{H_{1} \tilde{L}_{i}}^{2}+3\left(Y_{D^{\prime}}^{t} \Lambda_{D^{m}}^{\dagger} \Lambda_{D^{m}} \Lambda_{D^{j}}^{\dagger} m_{\tilde{L}}^{2}\right)_{j i}+3 \operatorname{Tr}(D)(E)_{q i} m_{H_{1} \tilde{L}_{q}}^{2},
\end{aligned}
$$

where

$\left(m_{H_{1} \tilde{L_{i}}}^{2}\right)=\left(m_{\tilde{L_{i} H_{1}}}^{2}\right)^{*}, D=Y_{D} Y_{D}^{\dagger}, T=Y_{U} Y_{U}^{\dagger}, E=Y_{E} Y_{E}^{\dagger}, \tilde{D}=Y_{D}^{\dagger} Y_{D}, \tilde{T}=Y_{U}^{\dagger} Y_{U}, \tilde{E}=Y_{E}^{\dagger} Y_{E}$,

and

$$
\begin{aligned}
s^{\prime}= & 2 g_{1}^{2}\left[\operatorname{Tr}\left(\Lambda_{D^{q}}^{\dagger} \Lambda_{D^{q}} m_{\tilde{Q}}^{2 t}\right)-\operatorname{Tr}\left(\Lambda_{E^{q}}^{\dagger} \Lambda_{E^{q}} m_{\tilde{L}}^{2}\right)+2 \operatorname{Tr}\left(\Lambda_{U^{q}}^{\dagger} \Lambda_{U^{q}} m_{\tilde{D}}^{2}\right)\right. \\
& \left.-2\left(m_{\tilde{U}}^{2}\right)_{k l} \operatorname{Tr}\left(\Lambda_{U^{l}}^{\dagger} \Lambda_{U^{k}}\right)+2\left(m_{\tilde{D}}^{2}\right)_{k l} \operatorname{Tr}\left(\Lambda_{D^{l}}^{\dagger} \Lambda_{D^{k}}\right)+\left(m_{\tilde{E}}^{2}\right)_{k l} \operatorname{Tr}\left(\Lambda_{E^{l}}^{\dagger} \Lambda_{E^{k}}\right)\right](59)
\end{aligned}
$$

for the soft SUSY breaking masses, the $s^{\prime}$-term comes from exclusion the U(1) FayetIliopoulos (FI) D-term by using its equation of motion then this renormalization gives rise to extra contributions proportional to the $\mathrm{U}(1)$ gauge coupling [7. To check our results,first we have calculated the full one loop R-parity violating RGEs, and have compared with ref 17, 21. Our results are the same as theirs. Moreover; to test the above method we have obtained the full two loop R-parity conserving RGEs which are consistent with results in ref [22. Finally, when we set all lepton number violating couplings to be zero we have obtained the full two loop the baryon number violating RGEs which we could compare with results in ref 14. We have realized that we have made some mistakes at previous paper; here we have generalised the latter results to include lepton number violating terms, and take the opportunity to correct some errors in Ref [14.

\section{Conclusions}

In this work, we have presented the full two-loop $\beta$-functions for all couplings for the RPV MSSM. In previous papers [14, 23, 24, we have studied the effects of some RPV couplings up two-loop RGEs corrections; there we have made a Form program to calculate some of two loop RPV $\beta$-functions as well as RPC $\beta$-functions. however we have not calculated explicitly the full two-loop $\beta$ functions for $R$ parity violating MSSM couplings.

Typically, we expect effects of the two loop $\beta$-functions make a difference of several percent in compare with effects of one loop on the standard running analysis such as Higgs physics and the scalar quark sector of the MSSM; however it is quite difficult to make consequential estimates of the size of the two-loop corrections without committing to a specific model. Moreover it is desirable from the point of view of consistency to use the full set of $\beta$-functions. In any event, we hope that the full set of $\beta$-functions for the most general R-parity violating version of the MSSM could be used in the future analysts.

\section{References}

[1] H. Dreiner, hep-ph/9707435 
[2] D. I. Kazakov, hep-ph/0012288

[3] L. E. Ibanez and G. G. Ross. Nucl. Phys., B368:3, 1992.

[4] P. Fayet, Phys. Lett. B 69 (1977) 489. G. R. Farrar and P. Fayet, Phys. Lett. B 76,575 (1978).

[5] L.E. Ibanez and G.G. Ross, Nucl. Phys. B 368 (1992) 3; Phys. Lett. B 260 (1991) 291; H.K. Dreiner, C. Luhn and M. Thormeier, Phys. Rev. D 73 (2006) 075007.

[6] H.K. Dreiner, hep-ph/9707435.

[7] B.C. Allanach, A. Dedes and H.K. Dreiner, Phys. Rev. D 69 (2004) 115002 [Erratum ibid. D 72 (2005) 079902].

[8] S. Davidson, M. Losada Nucl.Phys.B587:118-146,2000.

[9] I. Jack, D.R.T. Jones and C.G. North, Phys. Lett. B 386 (1996) 138; Nucl. Phys. B 486, (1997) 479.

[10] I. Jack, D.R.T. Jones and C.G. North, Nucl. Phys. B 473 (1996) 308.

[11] I. Jack, D.R.T. Jones and A.F. Kord, Phys. Lett. B 579 (2004) 180; Ann. Phys. 316 (2005) 213.

[12] B.C. Allanach, A. Dedes and H.K. Dreiner, The R parity violating minimal supergravity model, Phys. Rev. D 69 (2004) 115002 [Erratum ibid. D 72 (2005) 079902] hep-ph/0309196.

[13] B.C. Allanach, A. Dedes and H.K. Dreiner, Two-loop supersymmetric renormalization group equations including R-parity violation and aspects of unification, Phys. Rev. D 60 (1999) 056002 hep-ph/9902251.

[14] A.F. Kord JHEP12(2008)005

[15] ] I. Jack and D.R.T. Jones Phys. Lett. B415 (1997) 383

[16] I. Jack, D.R.T. Jones and A. Pickering, Phys. Lett. B432 (1997) 114

[17] L.V. Avdeev, D.I. Kazakov and I.N. Kondrashuk, Nucl. Phys. B510 (1998) 289

[18] D.R.T. Jones, Phys. Lett. B123 (1983) 45 ; V. Novikov et al, Nucl. Phys. B229 (1983) 381 ; V. Novikov et al, Phys. Lett. B166 (1986) 329 ; M. Shifman and A. Vainstein, Nucl. Phys. B277 (1986) 456

[19] I. Jack, D.R.T Jones, S.P. Martin, M.T. Vaughn and Y. Yamada, Phys. Rev. D 50, 5481 (1994)

[20] I. Jack, D.R.T. Jones and A. Pickering, Phys. Lett. B432 (1998) 114

[21] B. de Carlos and P.L. White, Phys. Rev. D 54 (1996) 3427; Phys. Rev. D 55 (1997) 4222.

[22] S.P. Martin and M.T. Vaughn, Phys. Rev. D 50 (1994) 2282 [Erratum ibid. D 78 (2008).

[23] A.F. Kord, Phys. Lett. B687 (2010)388-394.

[24] I. Jack, D.R.T. Jones and A.F. Kord, Phys. Lett. B 632 (2006) 703 http://www.liv.ac.uk/ dij/rpvbetas/. 